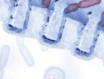

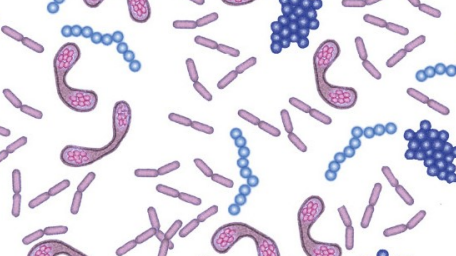
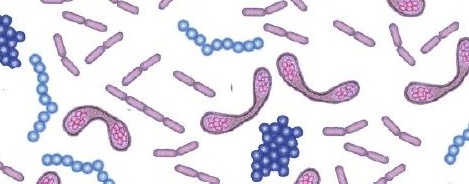

\section{The Role of Wound Healing in Oral Health:}

Metabolite Signatures and Microbes

Influence Re-Epithelialization

María Marcela Fernández Gutiérrez 


\section{Propositions}

1. The consequences of forgetting a toothbrush during a 2-week trip can be foreseen in a saliva sample.

(this thesis)

2. The modified Gompertz function, originally developed to model bacterial growth curves, can accurately describe in vitro re-epithelialization kinetics.

(this thesis)

3. Publishing results that support a null hypothesis avoids frustrations and spares resources.

4. Marketing and storytelling are essential skills for a successful scientific career.

5. Social media provide a rich source of information, but also facilitate the creation of dangerous misconceptions.

6. Humans are the biggest threat for our planet, but ironically also the only species that is able to save it.

7. Not vaccinating your children should be considered as negligence and thus, vaccination should be compulsory by law.

Propositions belonging to the thesis entitled

"The Role of Wound Healing in Oral Health:

Metabolite Signatures and Microbes Influence Re-Epithelialization"

María Marcela Fernández Gutiérrez

Wageningen, 16 March 2018 


\section{The Role of Wound Healing in Oral Health:}

Metabolite Signatures and Microbes Influence Re-Epithelialization

María Marcela Fernández Gutiérrez 


\section{Thesis Committee}

\section{Promotor}

Prof. Dr Michiel Kleerebezem

Personal chair at the Host-Microbe Interactomics Group

Wageningen University \& Research

\section{Other members}

Prof. Dr Geert F. Wiegertjes, Wageningen University \& Research

Prof. Dr Wim Teughels, KU Leuven, Leuven, Belgium

Prof. Dr Egjia Zaura, ACTA, Amsterdam, The Netherlands

Dr Marko de Jager, Phillips Research, Eindhoven, The Netherlands

This research was conducted under the auspices of the Graduate School (Advanced studies in Food Technology, Agrobiotechnology, Nutrition and Health Sciences). 


\title{
The Role of Wound Healing in Oral Health:
}

Metabolite Signatures and Microbes Influence Re-Epithelialization

María Marcela Fernández Gutiérrez

\author{
Thesis \\ submitted in fulfilment of the requirements for the degree of doctor \\ at Wageningen University \\ by the authority of the Rector Magnificus, \\ Prof. Dr A.P.J. Mol, \\ in the presence of the \\ Thesis Committee appointed by the Academic Board \\ to be defended in public \\ on Friday 16 March 2018 \\ at 4 p.m. in the Aula.
}




\section{María Marcela Fernández Gutiérrez}

The Role of Wound Healing in Oral Health: Metabolite Signatures and Microbes Influence Re-Epithelialization, 186 pages.

PhD thesis, Wageningen University, Wageningen, the Netherlands (2018)

With references, with summary in English

\section{ISBN 978-94-6343-733-2}

DOl: https://doi.org/10.18174/430645 
A mi familia 



\section{Table of Contents}

Chapter 1

Introduction

Chapter 2

Streptococcus salivarius MS-oral-D6 Promotes Gingival Re-Epithelialization in vitro

through a Secreted Serine Protease

\section{Chapter 3}

KREAP: An automated Galaxy Platform to Quantify Re-Epithelialization Kinetics

\section{Chapter 4}

High-Throughput Screening Model to Quantify Re-Epithelialization Kinetics

\section{Chapter 5}

Salivary Metabolite Signature Predicts in vitro Re-Epithelialization Kinetics and is Associated with Gingival Bleeding in Human Volunteers

\section{Chapter 6}

Identification of Oral Commensal Bacteria Related to Gingival Bleeding and Re-Epithelialization with Disease-Risk Signature Potential

\section{Chapter 7}

Differential Effects of Oral Bacteria on Gingival Cells Revealed by Cell-Based Assays

and Transcriptome Analysis

\section{Chapter 8}

Discussion

References

Summary

Acknowledgements

About the Author

List of Publications 



\section{Chapter}

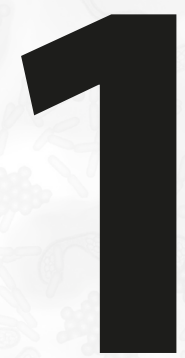

Introduction 


\section{Introduction}

Oral health is an essential component for overall health and well-being. The oral cavity plays an important role in the initiation of food digestion, the perception of taste, and in social interaction involving verbal and non-verbal communication'. In addition, decreased oral health has been shown to impact systemic health by increasing the risk of developing focal infections as well as other diseases, including atherosclerosis and diabetes mellitus ${ }^{1-4}$. Great efforts have been invested in the development of strategies for treatment and prevention of oral diseases. According to the Centers for Disease Control and Prevention (CDC), one of the greatest public heath achievements in the 20th century was water fluoridation which led to a sharp decline of dental caries especially among children. However, despite these efforts, caries and periodontal diseases are among the most common conditions worldwide, affecting more than 2.4 and 6.5 billion people worldwide, respectively ${ }^{5,6}$. Therefore, there is an increased interest in the development of new strategies that aim to understand, maintain and promote the healthy status of the oral cavity.

\section{The Oral Cavity}

\section{Structural organization of the oral mucosa and barrier function}

The oral cavity is lined by stratified squamous epithelium that constitutes a structural barrier between the internal and external environments, and protects the underlying tissues against mechanical and chemical damage, bacterial infection, and loss of fluids ${ }^{7}$. The oral mucosa differs structurally according to its location and function. The soft palate, cheeks, lips, ventral tongue, and the floor of the oral cavity (i.e. lining mucosa) consist of non-keratinized epithelium followed by a basal lamina, a lamina propia, and a submucosal layer that provides flexibility in order to chew, swallow, and speak (Fig. $\mathbf{1}^{7,8}$. In regions such as the gingiva and the hard palate (i.e. masticatory mucosa), the submucosa is not present and the lamina propia is directly attached to the periosteum of the bone, providing a firm and inelastic connection. The gingiva and hard palate are comprised of keratinized squamous epithelium that can resist the mechanical forces and abrasion associated with mastication ${ }^{7-9}$.

Keratinocytes undergo a well-defined differentiation program that results in the expression of structural proteins that help maintain the architectural integrity of the epithelial tissues and its barrier function. Cell-cell attachments are mediated by a specialized complex of transmembrane proteins called desmosomes ${ }^{7,10}$. Desmosomal cadherin proteins provide a link to the intracellular keratin filaments, which comprise the major cytoskeletal component of the epithelial cells next to the actin filaments and microtubules ${ }^{11}$. Finally, attachment of the cells to the extracellular matrix of the basal lamina is mediated by multiprotein junctional complexes called hemidesmosomes, which are critical for cell polarization, spatial organization, and tissue architecture ${ }^{7,12}$. The proliferation to differentiation switch is tightly controlled and compartmentalized into the basal and suprabasal layers, respectively. However, during normal wound healing, mitosis can occur in the upper differentiating layers to re-establish tissue integrity? 

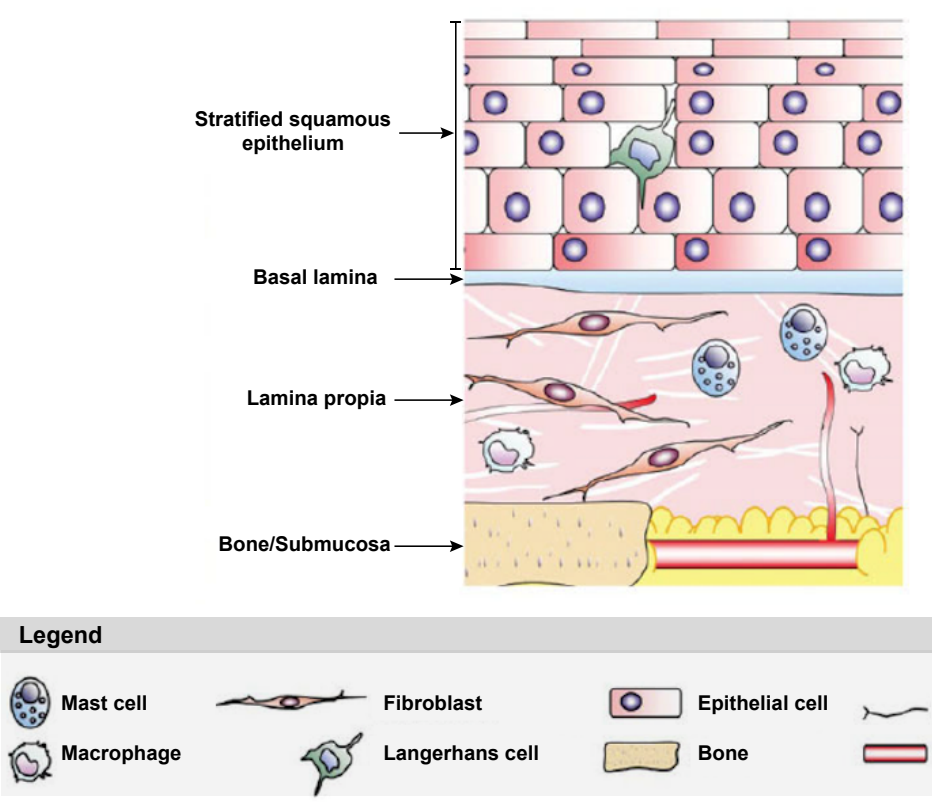

Nerve ending

Blood vessel

Figure 1. Schematic representation of the oral mucosa. The oral epithelium consists of several layers of keratinized or non-keratinized epithelial cells as well as few Langerhans cells. The lamina propia is separated from the epithelium by the basal lamina and include mast cells, macrophages, fibroblasts, nerves, and blood vessels, which are surrounded by extracellular matrix proteins. The submucosa consists mainly of adipose tissue. In the areas where the submucosa is not present, the lamina propia is directly connected to the bone. Adapted from Glim et al. (2013) ${ }^{9}$ with permission of Wiley Online Library.

\section{Active role of the oral mucosa in host defence}

Apart from providing a physical barrier, the oral mucosa protects the host against pathogenic bacteria through diverse mechanisms. Firstly, continuous cell renewal and shedding of cells from the upper layers of the epithelium minimizes bacterial colonization and thereby, protects the host from invading microorganisms ${ }^{13}$. Secondly, epithelial tissues protect the host through secretion of antimicrobial peptides, which have a broad specificity and activity against bacteria, fungi, and viruses ${ }^{11,14} . \beta$-defensins and $L L-37$ are cationic antimicrobial peptides expressed by the oral mucosa that aggregate on the negatively charged microbial surface, resulting in the formation of pores that disrupt the bacterial membrane integrity ${ }^{15}$. Besides their direct antimicrobial activity, these peptides have been shown to have signalling potential and serve as cross-talk between the innate and the acquired immune responses. For instance, LL-37 serves as a neutrophil chemoattractant ${ }^{16}$, whereas $\beta$-defensins act as chemoattractant for dendritic cells and memory $\mathrm{T}$ cell $\mathrm{s}^{17}$, and enhance both cellular and humoral immune responses when administered as adjuvants ${ }^{18}$. Thirdly, epithelial cells respond to bacteria through activation of Toll-like receptors, which recognize specific molecular patterns in microbial components that ultimately lead to the activation of the innate immunity through expression of pro-inflammatory cytokines and chemokines ${ }^{19}$. Lastly, immune cells recruited to or residing in the oral epithelium (Fig. 1) such as polymorphonuclear leukocytes (PMNs) and Langerhans cells, respectively, limit biofilm formation and protect the host from pathogenic microorganisms. Among the PMNs, neutrophils are key effector cells in innate immunity against bacteria and are recruited in high numbers to the oral mucosa. The junctional epithelium, a specialized tissue that surrounds the tooth, generates 
a gradient of chemotactic interleukin-8 (IL-8) that results in constant recruitment of neutrophils to the gingival sulcus ${ }^{20}$. Neutrophils can limit biofilm formation using several host-defence mechanisms which include phagocytosis, release of reactive oxygen species (ROS) in combination with a wide range of proteolytic and antimicrobial enzymes, and generation of neutrophil extracellular traps (NETs) consisting of chromatin and proteases that trap and kill bacteria ${ }^{21}$. Furthermore, Langerhans cells are dendritic cells that migrate out of the tissue for antigen presentation, eliciting acquired immune responses that provide long-term recognition of foreign antigens ${ }^{13}$. Taken together, the oral epithelium responds to the presence of bacteria and subsequent signalling amplifies their response through stimulation of immune cells such as dendritic cells, T cells, and macrophages, which provide a link between the innate immune responses of the epithelium and the acquired immunity.

\section{Saliva: Main Functions and Constituents}

Human saliva contains a mixture of secretions derived from several salivary glands including the parotid, submandibular, sublingual and other minor glands beneath the oral mucosa as well as gingival crevicular fluid ${ }^{22}$. The submandibular and sublingual glands generate about $70 \%$ of the saliva and are responsible for the viscous nature of this biofluid which results from the presence of highly glycosylated mucins in these secretions. In contrast, the saliva generated by the parotid gland accounts for $20 \%$ of the saliva and it is characterized by a watery composition due to the lack of mucins ${ }^{23,24}$. On average, a healthy person has a salivary flow rate of $0.3-0.4 \mathrm{ml} / \mathrm{min}$, generating around 0.75 to $1.5 \mathrm{~L}$ of saliva per day 23,25 .

Saliva facilitates bolus formation by moistening food to ensure comfortable swallowing and allows soluble food-derived molecules to reach taste buds containing gustatory cell ${ }^{24,26}$. Taste perception is modulated by carbonic anhydrase $\mathrm{VI}$ (referred to as gustin), a zinc-metalloprotein that constitutes about $3 \%$ of parotid saliva protein ${ }^{27}$. In addition, saliva contains a myriad of proteins, peptides and inorganic constituents which play a role in essential biological processes such as food digestion, and that are crucial for the maintenance of different aspects of oral health, including defence against pathogenic microorganisms, teeth protection and re-mineralization, and wound repair (Fig. 2) 28,29.

In combination with the mechanical forces provided by the teeth, salivary enzymes play an important role in the initiation of food digestion ${ }^{28}$. For example, salivary a-amylase, protease, and lipase aid in the degradation of dietary starches, proteins, and fats, respectively. Next to the digestive enzymes, saliva contains a variety of proteins and peptides (e.g. agglutinin, cystatins, lactoferrin, lactoperoxidase, immunoglobulins, and lysozyme) that together with the antimicrobial peptides produced by the oral mucosa, protect the hard and soft surfaces of the oral cavity against pathogenic microorganisms 28,30 . Inorganic compounds, such as bicarbonate and phosphate, provide saliva with buffering capacity to neutralize acid in the oral cavity that would otherwise demineralize the hydroxyapatite component of the enamel. Specific proteins, including statherin and proline-rich proteins (PRP), participate in the formation of a salivary pellicle that protects the teeth from demineralization and at the same time helps restore enamel lesions ${ }^{31}$. Moreover, mucins and proline-rich glycoproteins (PRG) in saliva provide lubrication to the teeth and oral mucosa, which counteracts tooth wear and tissue abrasion, respectively ${ }^{25,28}$. Finally, saliva contains numerous growth factors (Table 1) and antimicrobial peptides (e.g. histatin 1 and histatin 2) that stimulate oral wound healing and therefore, play an important role in the maintenance of epithelial integrity and barrier function ${ }^{29}$. 


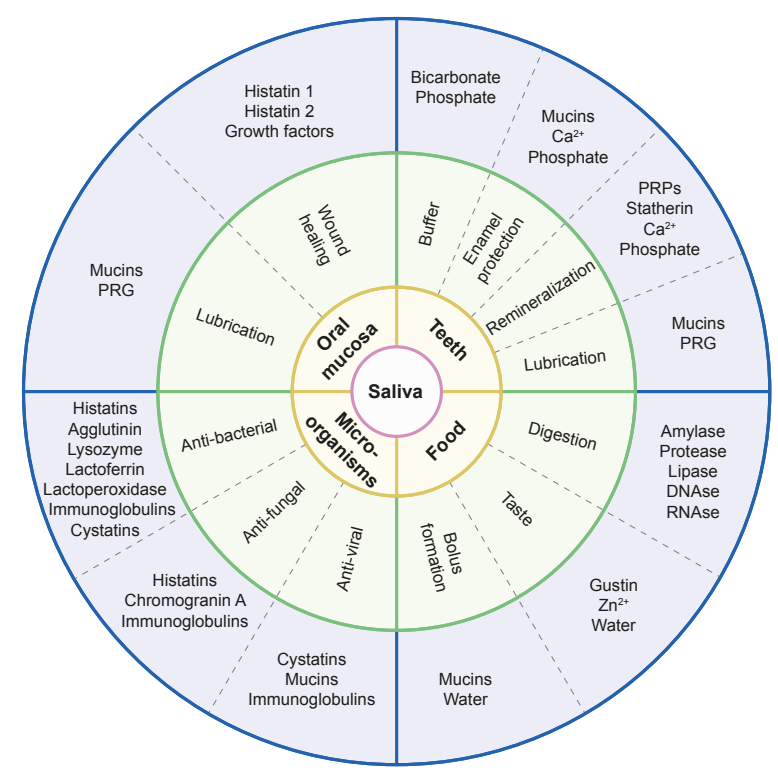

Figure 2. Saliva main functions and constituents. Schematic representation of salivary constituents involved in various functions. Adapted from Amerongen et. al. $(2002)^{28}$ with permission of Wiley Online Library.

Table 1. Growth factors in saliva. Concentrations of growth factors in human saliva in comparison with plasma. Adapted from Oudhoff (2001) ${ }^{24}$.

\begin{tabular}{lccc}
\hline Growth factor & Abbreviation & Saliva $(\mathbf{n g} / \mathbf{m l})$ & Plasma $(\mathbf{n g} / \mathbf{m l})$ \\
\hline Epidermal growth factor & EGF & 0.9 & 0.2 \\
\hline Nerve growth factor & NGF & 0.9 & 0.1 \\
\hline Vascular endothelial growth factor & VEGF & 1.4 & 0.5 \\
\hline Fibroblast growth factor & FGF & $<0.001$ & 0.2 \\
\hline Insulin-like growth factor & IGF & 0.4 & 170 \\
\hline Transforming growth factor a & TGFa & 5.6 & 0.03 \\
\hline Transforming growth factor $\beta$ & TGF $\beta$ & 0.024 & 2.0 \\
\hdashline Tumor necrosis factor a & TNFa & 0.003 & 0.008 \\
\hdashline Insulin & - & 0.2 & 925 \\
\hline
\end{tabular}

Taken together, the salivary proteome has been studied quite extensively. However, saliva is also a rich source of metabolites or small molecules that unlike the salivary proteome, little is known about their role in the maintenance of oral health and/or mucosal integrity. More than 2,000 salivary metabolites have been identified and catalogued in the Human Metabolome Database (HMDB) (http://www.hmdb.ca/), providing the most up to date and comprehensive library of the human metabolome ${ }^{32}$. 


\section{The Oral Microbiome}

The warm and moist environment of the oral cavity in combination with host-derived nutrients, such as saliva proteins, glycoproteins, and gingival crevicular fluid (GCF) support the growth of many microorganisms ${ }^{28,33}$. Furthermore, the heterogeneous environment of the oral cavity provides niches that offer different conditions (e.g. pH, oxygen and nutrient availability) that select for specific microbial communities ${ }^{33,34}$. These distinct niches include the hard surfaces of the teeth, keratinized and non-keratinized mucosal epithelium, the tongue, and the gingival sulcus. Moreover, the teeth provide the only non-shedding surfaces in the human body that facilitate the formation of biofilms (plaque) and ensure microbial persistence ${ }^{33}$.

Bacteria in the oral cavity were among the first to be described back in the $17^{\text {th }}$ century by Antonie van Leeuwenhoek, a Dutch scientist that is often referred to as the "Father of Microbiology". In his report to the British Royal Society, he describes various forms of microorganisms in dental plaque, which he originally referred to as animalcules (from Latin animalculum = "tiny animal" ${ }^{35}$. When techniques for culturing bacteria were developed, much effort was invested in the characterization of the oral microbiota. However, many oral bacteria require strict anaerobic conditions, complex growth media, and long incubation times to grow, which limited the capacity of traditional culture techniques to adequately represent the bacterial community of the oral cavity. Notably, it is currently estimated that approximately $30 \%$ of the oral bacterial species remains uncultured to date ${ }^{36}$.

Culture-independent molecular methods, primarily using 165 rRNA gene-based approaches, have enabled significant progress in the elucidation of the composition of the oral ecosystem ${ }^{37-40}$. More than 700 bacterial species inhabit the oral cavity and make up the oral microbiome ${ }^{36}$. These species include members of seven phyla that are present in variable abundance and encompass a variety of different genera: Actinobacteria (genera Corynebacterium, Rothia, Actinomyces), Bacteroides (genera Prevotella, Capnocytophaga, Porphyromonas), Firmicutes (genera Streptococcus, Veillonella), Fusobacteria (genus Fusobacterium), Proteobacteria (genera Neisseria, Haemophilus), Spirochetes (genus Treponema) and candidate division $\mathrm{TM}^{77,39}$.

Intriguingly, despite the daily physical and chemical perturbations that result from external factors, such as intake of food, personal hygiene measures, and others, the oral microbiota has been shown to be relatively stable within an individual over long periods of time $e^{41}$. In addition, data from the Human Microbiome Project ${ }^{42}$ revealed that the oral cavity has the largest set of shared taxa among unrelated healthy individuals ("core microbiome") when compared to other hostassociated habitats such as the vagina, the skin or the gastrointestinal tract ${ }^{43,44}$.

\section{Oral Health and Disease}

Oral health can be defined as the ability of an individual to achieve homeostasis by adapting to the changing conditions of the oral ecosystem, a term known as allostasis (Fig. $\mathbf{3}^{45}$. In healthy conditions, the host lives in a relatively stable and symbiotic relationship with its resident microbes. The host provides the commensal bacteria with a suitable niche and essential nutrients to support their growth. In turn, the commensal bacteria actively contribute to oral health by preventing pathogens from colonizing the oral cavity and promoting a normal development of the host immune system, including tolerance to self-antigens and commensal microbes ${ }^{33,46}$. Using mice models, commensal bacteria have also been shown to induce expression of chemokine (C-X-C motif) ligand 2 (CXCL2) which activates the CXCR2 receptor, a 
key chemotactic receptor that facilitates neutrophil recruitment to the oral tissues contributing to the maintenance of homeostasis ${ }^{47}$.

However, a substantial change in a local environment may result in a dysbiotic shift and loss of community balance. Some of these changes may include salivary gland dysfunction, poor oral hygiene, gingival inflammation, and lifestyle choices such as smoking and increased frequency of sugar intake ${ }^{36,48}$. When the stability of the ecosystem is disturbed, exogenous microorganisms may be able to colonize the site, or previously minor members of the microbial community may subvert the host restraint mechanisms, increase in proportion, and participate in the development of disease (Fig. 3 ) $^{33}$.

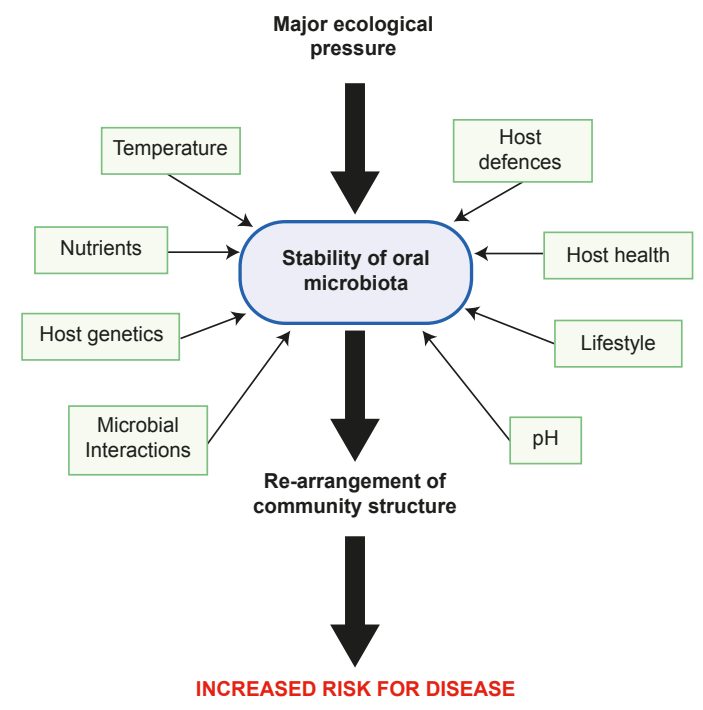

Figure 3. Oral allostasis. Host factors that influence microbial stability, composition and activity. A major ecological pressure can result in a rearrangement of the resident microbial community and an increased risk for diseases. Adapted from Marsh and Devine $(2011)^{33}$ with permission of Wiley Online Library.

\section{Dental caries}

Dental caries is one of the most common infectious diseases in humans ${ }^{49}$. Increased frequency of sugar intake or a reduction in the salivary flow, result in a more frequent and longer exposure of the oral microbiota to lower pH levels, which drives the selection of more acid-tolerant and acid producing bacteria, at the expense of bacteria that grow in neutral conditions ${ }^{36}$. Cariogenic bacteria can metabolize carbohydrates through fermentation and produce lactic acid that causes irreversible demineralization of the hard tissues of the tooth (enamel, dentin and cementum), resulting in a carious lesion ${ }^{49}$. Dental caries is primarily associated with high numbers of Streptococcus mutans and other related streptococci (Streptococcus oralis, Streptococcus mitis, Streptococcus anginosus) at the diseased site. Other genera associated with dental caries include Rothia, Neisseria, Selenomonas, Actinomyces, Lactobacillus, and Veillonella ${ }^{49,50}$. From these microorganisms, Lactobacillus are thought to be involved in the progression rather than the initiation of the disease, whereas Actinomyces and Veillonella are likely to be enriched in carious lesions as a result of their ability to consume lactate, of which the levels are highly elevated in these lesions ${ }^{49}$. 


\section{Periodontal diseases}

Periodontal diseases refer to the common inflammatory disorders of gingivitis and periodontitis ${ }^{6}$. Apart from being detrimental to oral health, periodontal diseases have been associated with adverse pregnancy outcomes and the development of diseases such as atherosclerosis and diabetes mellitus ${ }^{3,451,52}$ Moreover, periodontal diseases favour the entry of bacteria into the bloodstream which may result in the development of focal infections, including endocarditis and pneumonia².

\section{Risk factors and development of periodontal diseases}

Social and behavioural factors as well as systemic and genetic factors influence the manifestation and progression of periodontal diseases. For instance, smoking, vitamin C deficiency and presence of systemic diseases (e.g. osteoporosis, acquired immune deficiency syndrome (HIV/AIDS), and diabetes) increase the risk for developing the disease $e^{53-56}$. Furthermore, genetic diseases such as Chediak-Higashi syndrome, Papillon-Lefèvre syndrome, and chronic/cyclic neutropenia are associated with periodontitis as they affect neutrophil activity or recruitment, essential for maintenance of periodontal health ${ }^{57}$.

Gingivitis is caused by accumulation of dental plaque (biofilm) on the teeth adjacent to the gingiva that results in mild inflammation ${ }^{6}$. The local inflammation causes an increased flow of gingival crevicular fluid (GCF) and micro-ulcerations of the sucular epithelium. This leads to blood leakage into the GCF and subsequent oxygen deprivation. The anaerobic environment in combination with the enriched nutrient source (i.e. GCF and haemin present in blood) are suitable conditions to promote the growth of strict anaerobic periodontal pathogens such as Porphyromonas gingivalis, which contribute to the development of the disease (see section below) ${ }^{36}$. Gingivitis can be reversible with adequate oral hygiene, but in susceptible individuals it can progress to periodontitis. This disorder is characterized by a dysregulated host immune response that can eventually result in irreversible reabsorption of the alveolar bone, destruction of the connective tissue, and tooth loss 6 .

\section{Porphyromonas gingivalis: A key periodontal pathogen}

Porphyromonas gingivalis is a Gram-negative anaerobic asaccharolytic bacterium that relies in the degradation of proteins for its growth and is considered one of the major pathogens in periodontitis ${ }^{58,59}$. P. gingivalis is a member of the so-called "red complex" that together with Treponema denticola and Tannerella forsythia are associated with advanced periodontal disease $^{60}$. P. gingivalis produces several virulence factors including, lipopolysaccharide (LPS), capsule, fimbriae, proteases, and outer membrane vesicles ${ }^{61,62}$. Some of these virulence factors are used by the bacterium for nutrient uptake and survival, but also to deregulate the host innate immune and inflammatory responses. P. gingivalis adheres to the host cell surface using fimbriae and subsequently, the bacterium is internalized via lipid rafts into early phagosomes. Thereafter, P. gingivalis activates autophagy and suppresses apoptosis to provide a suitable niche for survival and replication hidden from the host's immune surveillance ${ }^{59}$. Secreted cysteine proteolytic enzymes called gingipains have the ability to inactivate several host components including, complement system proteins, cytokines (IL-1 $1 \beta$, IL-6, IL-8, IL-12, TNFa, IFN $\gamma$ ), antimicrobial peptides, integrins, and collagen ${ }^{63}$. Moreover, $P$. gingivalis has evolved mechanisms to evade neutrophil clearance, leading to accumulation of these immune cells in the periodontal tissues. The oxidative responses mounted by the neutrophils subsequently result in tissue damage and in severe cases, bone loss ${ }^{20,64}$. Moreover, $P$. gingivalis contributes 
to the development of periodontitis by inducing high levels of pro-inflammatory cytokines, such as IL-1 $\beta$ and IL-6, and stimulating the production of receptor activator of NF-KB ligand (RANKL) ${ }^{59}$. Overexpression of RANKL causes activation of osteoclasts, which in turn mediate bone loss by increasing their resorptive activity exacerbating the disease $\mathrm{e}^{65}$. Taken together, periodontitis is a multi-factorial disease whose inflammatory responses are determined by a complex interplay between the host immune system and the periodontal pathogens ${ }^{66}$.

\section{Oral Wound Healing}

Oral wounds can result from daily activities (e.g. tooth brushing, chewing, eating), but also from surgery, trauma or presence of inflammatory conditions such as periodontal diseases. When the epithelial integrity is compromised, rapid reepithelialization is necessary to restore homeostasis and prevent infections that could otherwise promote inflammation and tissue damage ${ }^{67}$. Thus, wound healing is an essential biological process orchestrated by a highly regulated signalling network that involves numerous growth factors, cytokines and chemokines in four main overlapping phases (Fig. 4): clot formation, inflammation, re-epithelialization, and tissue remodelling ${ }^{68-71}$.

\section{Phases of wound healing}

Clot formation is initiated by damage signals (e.g. ATP, $\mathrm{Ca}^{2+}, \mathrm{H}_{2} \mathrm{O}_{2}$ ) that trigger the activation, adhesion and aggregation of platelet $^{70,72}$. Platelet-secreted factors such as platelet-derived growth factor (PDGF), epidermal growth factor (EGF) and transforming growth factor- $\beta$ (TGF $\beta$ ) stimulate fibrin formation and its deposition, which together with the aggregated platelets, form a plug at the site of the injury that limits further blood loss and pathogen invasion ${ }^{68,71}$. In the inflammatory phase, neutrophils and other leukocytes are recruited to the wound to clear the site from bacteria or other pathogenic microorganisms. Subsequently, monocytes arrive, mature into macrophages and remove cell debris and dead neutrophils by phagocytosis ${ }^{68}$. Inflammation is normally accompanied by angiogenesis, which facilitates the migration of immune cells towards the wound $\mathrm{d}^{70}$. In addition, the immune and epithelial cells also produce growth factors and cytokines, which initiate the proliferative phase of wound repair ${ }^{71}$. Epithelial cells at the wound edge migrate and proliferate into the wounded area to re-establish the original cell numbers, a process known as re-epithelialization ${ }^{69}$. In normal wound healing, members of the epidermal growth factor family such as EGF and transforming growth factor a (TGFa) stimulate re-epithelialization via recognition and activation of the EGF receptor (EGFR) on epithelial cells $s^{71,73}$. Fibroblasts are also recruited to the wound where they differentiate into myofibroblasts. Fibroblasts and myofibroblasts secrete collagen and extracellular matrix (ECM) proteins, which together with new vasculature forms the granulation tissue ${ }^{68}$. As the edges of the wound come together, the cell migration and proliferation ceases. The immune cells leave the area and the granulation tissue is removed by metalloproteinases. Finally, the ECM is remodelled to promote the formation of scar tissue $^{70}$. 


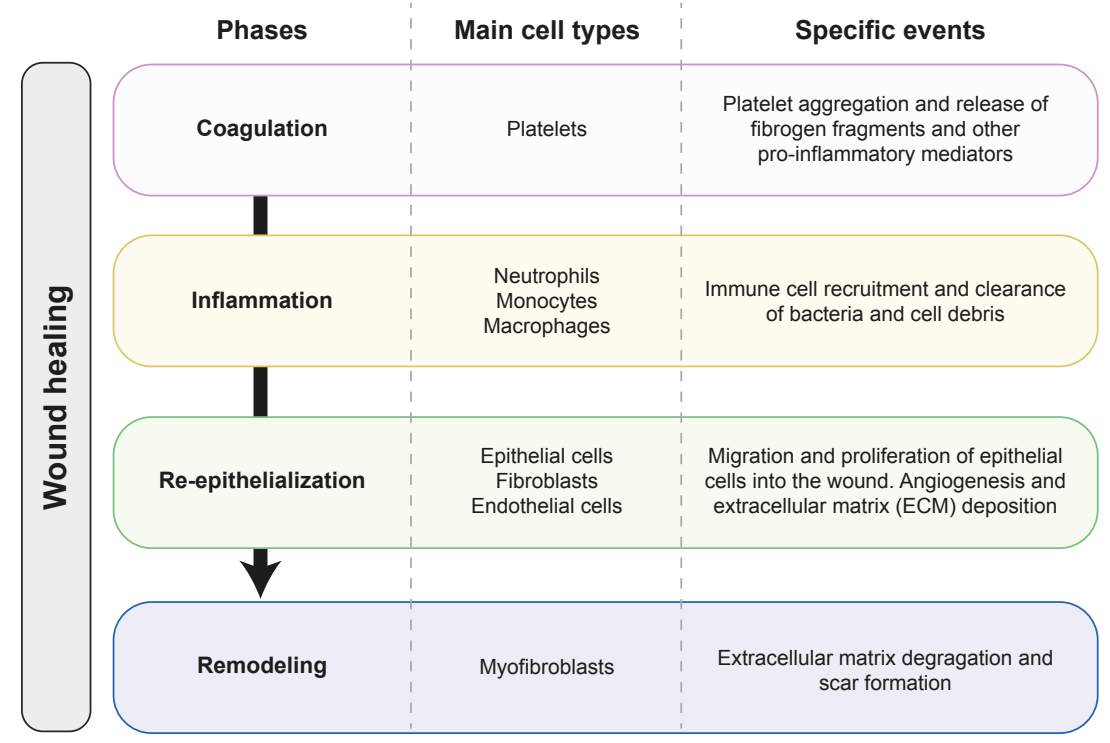

Figure 4. Wound healing. Specific events and cell types involved in wound healing.

\section{Oral wound healing vs Skin wound repair}

Unlike skin injuries, the oral mucosa is characterized by its rapid repair rate with minimal scar formation ${ }^{74}$. Faster wound healing in the oral cavity has been attributed to several factors, including a higher turnover rate of the oral epithelium, better vascularization, and presence of salivary constituents that promote wound repair ${ }^{29,75}$. Moreover, in comparison with skin, oral wounds have significantly lower levels of immune cell infiltrate and therefore, a lower expression of pro-inflammatory cytokines such as IL-6, IL-8, and transforming growth factor- $\beta$ (TGF $\beta 1)^{9,74}$. The reduced inflammatory response in combination with the abovementioned factors, are key for the superior wound healing capacity of the oral epithelium.

\section{Chronic wounds: role of periodontal pathogens}

Chronic wounds fail to proceed through the normal stages of wound repair in a timely and orchestrated manner, but instead remain in a chronic inflammatory state. Systemic conditions and local stimuli, such as bacterial infection, may contribute to perpetuate the inflammatory phase of wound repair, generating a cascade of immune and tissue responses that results in delayed or impaired wound healing ${ }^{76}$. Individuals that undergo hematopoietic stem cell transplant (HSCT) or chemo- or radiation therapy for cancer treatment often suffer from mucositis characterized by painful chronic ulcerations that cause the patient substantial discomfort ${ }^{77}$. Similarly, active periodontitis causes epithelial loss of barrier integrity and presence of periodontal pathogens promotes further dysbiosis and tissue disruption ${ }^{11,36}$. Notably, $P$. gingivalis has been identified as a positive predictor for oral mucositis after $\mathrm{HSCT}^{78}$ and has been shown to strongly inhibit re-epithelialization in several in vitro studies ${ }^{79,80}$. 


\section{Aims and Outline of this Thesis}

Although the processes that underlie oral diseases have been extensively studied, the biological processes involved in the maintenance of a healthy oral ecosystem remain poorly understood. We hypothesized that maintenance of epithelial integrity and barrier function is a key aspect that supports oral health. Hence, the overall goal of this thesis was to study the interactions between the oral mucosa and different components of the oral ecosystem (i.e. oral commensal bacteria and salivary metabolites) that could influence re-epithelialization kinetics. Understanding these interactions may lead to innovative strategies to support oral health by promoting epithelial integrity and barrier function. The study described in this thesis was performed within a framework of the project "Novel Strategies to Promote Oral Health" funded through Top Institute of Food and Nutrition (TIFN).

Scratch assays are used to study the influence of bioactive compounds on re-epithelialization in vitro. These assays rely on the introduction of a scratch into a confluent cell monolayer that is monitored over time by microscopy. However, scratch assays are generally executed at low-throughput and lack the capability to quantitatively describe the re-epithelialization process. To this end, our first aim was to develop a high-throughput screening model to measure re-epithelialization kinetics in vitro, which we described in Chapter 2. The scratch assay uses automated microscopy and image segmentation in combination with a mathematical model that describes the re-epithelialization kinetics of gingival cells. Using this assay, we screened 39 lactic acid bacteria typically found in the oral cavity for their capacity to influence re-epithelialization kinetics. In addition, we identified a secreted protease of Streptococcus salivarius MS-oral-D6 that is responsible for the enhanced re-epithelialization observed with this strain. In Chapter 3, we developed and implemented the Kinetic ReEpithelialization Analysis Pipeline (KREAP) into a toolbox in Galaxy, providing an open-source, web-based platform that integrates multiple image processing and data extraction software tools into a workflow. Moreover, in Chapter 4, we provide an optimized and detailed protocol to perform the high-throughput assay using oral and skin cell lines. Crucial steps, trouble-shooting, and limitations are identified and described in detail.

The salivary proteome has been subject of extensive studies and in addition, human saliva has been described to contain numerous growth factors as well as antimicrobial peptides that can stimulate oral wound healing. Nevertheless, the role of the salivary metabolome in the maintenance of epithelial integrity remains unexplored. Hence, our second aim was to identify a metabolite signature that could predict the re-epithelialization capacity of a saliva sample. In Chapter 5 we measured the re-epithelialization kinetics of gingival cells upon treatment with a subset unstimulated saliva samples collected during an experimental gingivitis study conducted with healthy individuals. The metabolite profiles of the saliva samples were used to perform elastic net regression with stability selection to identify a metabolite signature related with the re-epithelialization kinetics measured in vitro. Furthermore, we explored the connection of the observed and/or predicted re-epithelialization capacity of the saliva samples with the gingival bleeding scores measured in the participants during the development of mild gingivitis. Gingival bleeding scores are used clinically to assess the inflammatory state of the periodontal tissues.

Oral health is determined by the complex and stable interplay between the host immune defences and the resident microorganisms. However, a substantial change in the ecosystem can disrupt the stability and lead to microbial community re-arrangement that predispose to disease, such as gingivitis and periodontitis. Our third aim consisted of investigating the associations of commensal oral bacteria with the gingival bleeding scores measured in a healthy cohort, and to 


\section{0}

explore the relationship of these associations with their effect on gingival re-epithelialization. In Chapter 6, we used elastic net regression to identify operational taxonomical units (OTUs) associated with gingival bleeding. Representative species of the identified OTUs were screened for their influence on re-epithelialization in vitro. Additionally, we validated the associations of the selected species with gingival bleeding using redundancy analysis (RDA) in a different cohort during the development of mild gingivitis in a two-week experimental gingivitis challenge. Finally, our fourth aim was to explore the molecular mechanisms underlying the interactions between the oral mucosa and specific oral commensal species that result in enhanced re-epithelialization in vitro. To this end, in Chapter 7 we used a combination of cell-based assays and transcriptome analysis of gingival cells exposed to Actinomyces oris, Actinomyces viscosus, Veillonella parvula and Fusobacterium nucleatum subsp. animalis during the re-epithelialization assay.

Chapter 8, summarizes the main findings of the thesis and discusses its contribution to the field of oral health. Moreover, we address several remaining questions and identify future avenues towards the development of second-generation probiotics or bioactive compounds that may support epithelial integrity and barrier function. 


\title{
Chapter
}

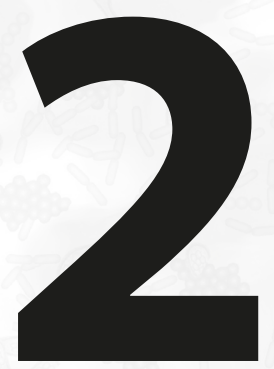

\section{Streptococcus salivarius MS-oral-D6 Promotes Gingival Re-Epithelialization in vitro through a Secreted Serine Protease}

\author{
Marcela M. Fernandez-Gutierrez ${ }^{1,2}$, \\ Peter P. J. Roosjen ${ }^{3}$, \\ Eveline Ultee ${ }^{2}$, \\ Maarten Agelink², \\ Jacques J.M. Vervoort ${ }^{4}$, \\ Bart Keijser ${ }^{1,5,6}$, \\ Jerry M. Wells' \\ Michiel Kleerebezem ${ }^{1,2^{*}}$
}

\footnotetext{
${ }^{1}$ TI Food and Nutrition, Nieuwe Kanaal 9-A, 6709 PA, Wageningen, The Netherlands.

${ }^{2}$ Host-Microbe Interactomics Group, Department of Animal Sciences, Wageningen University \& Research, De Elst 1, 6708 WD, Wageningen, The Netherlands.

${ }^{3}$ Laboratory of Geo-Information Science and Remote Sensing, Wageningen University \& Research, Droevendaalsesteeg 3, 6708 $\mathrm{PB}$, Wageningen, The Netherlands.

${ }^{4}$ Biochemistry Group, Department of Agrotechnology and Food Sciences, Wageningen University \& Research, Stippeneng 4, 6708 WE, Wageningen, The Netherlands.

${ }^{5}$ TNO Microbiology and Systems Biology, Utrechtseweg 48, $3704 \mathrm{HE}$, Zeist, The Netherlands.

${ }^{6}$ Department of Preventive Dentistry, Academic Centre for Dentistry Amsterdam, University of Amsterdam and Vrije Universiteit Amsterdam, Gustav Mahlerlaan 3004, 1081 LA, Amsterdam, The Netherlands.
} 
22 | Chapter 2

Gingival re-epithelialization represents an essential phase of oral wound healing in which epithelial integrity is re-established. We developed an automated high-throughput re-epithelialization kinetic cell-based model, using the gingival epithelial cell line Ca9-22. The model was employed to screen 39 lactic acid bacteria, predominantly including oral isolates, for their capacity to accelerate gingival re-epithelialization. This screen identified several strains of Streptococcus salivarius that stimulated re-epithelialization. Further analysis revealed that $S$. salivarius strain MS-oral-D6 significantly promoted re-epithelialization through a secreted proteinaceous compound and subsequent experiments identified a secreted serine protease as the most likely candidate to be involved in re-epithelialization stimulation. The identification of bacteria or their products that stimulate gingival wound repair may inspire novel strategies for the maintenance of oral health. 


\section{Introduction}

Certain members of the lactic acid bacteria (LAB), in particular the lactobacilli, have been widely used as probiotics in animal feeds and human products as they are generally recognized as safe (GRAS status) by The American Food and Drug Administration (FDA). Probiotics are "live microorganisms that when administered in adequate amounts, confer a health benefit on the host" ${ }^{\prime \prime 1}$. Probiotics have been reported to exert numerous beneficial effects on gut health, for example by antagonizing the growth of pathogenic bacteria ${ }^{82}$, promoting host-microbe homeostasis through modulation of immunity ${ }^{83,84}$, alleviating symptoms of lactose intolerance ${ }^{85}$, enhancing mucosal barrier function ${ }^{86-88}$, and promoting intestinal epithelial survival and growth ${ }^{89,90}$. Currently, there is an increasing interest in exploring the beneficial health effects of these probiotic bacteria in other body-sites such as the skin ${ }^{91}$, the urogenital tract ${ }^{92,93}$ and the oral cavity ${ }^{94}$. Studies on the beneficial effects of $L A B$ in the oral cavity have explored their ability to persist in this niche ${ }^{95,96}$, their antimicrobial activity against cariogenic and periodontal pathogens ${ }^{97,98}$, their capacity to diminish malodour ${ }^{99}$, and their ability to promote host-microbe homeostasis ${ }^{100}$. However, to our knowledge, there are no studies reporting beneficial effects on gingival wound healing.

The oral gingiva consists of stratified squamous epithelium that serves as a barrier between the external environment and the underlying tissue. Upon disruption of the barrier integrity, the processes involved in clearance of infection and renewal of damaged cells are immediately initiated. Healing of acute wounds occurs in four overlapping phases: clot formation, inflammation, re-epithelialization, and tissue remodelling ${ }^{68-71}$, regulated by a complex signalling network that involves numerous growth factors, cytokines and chemokines. Wounds that fail to proceed through the normal stages of wound repair in a timely and orchestrated manner result in chronic wounds, which are often colonized by bacteria that may contribute to the delayed or incomplete healing process by perpetuating inflammatory responses. This is the case in periodontitis, a bacterial infection characterized by chronic inflammation of the periodontal tissue that ultimately leads to destruction of the connective tissue and subsequent bone and tooth loss ${ }^{101,102}$. Prompt re-epithelialization is considered as one of the key parameters for optimal wound repair and prevention of chronic infections ${ }^{67}$. Re-epithelialization is driven by the proliferation and migration of epithelial cells into the site of injury to re-establish cell-to-cell contacts and close the wound. Scratch assays have been widely used as an in vitro model to study the influence of particular compounds on re-epithelialization. Such assays consist of the mechanical introduction of a scratch ("wound") into a confluent epithelial cell monolayer and following the re-epithelialization of that scratch by the acquisition of microscopic images over time $^{103}$. However, these assays tend to be executed at low throughput and in general suffer from poor reproducibility ${ }^{104}$ and absence of real-time kinetic data. Here we describe the development of a high-throughput scratch assay using liveautomated fluorescence microscopy, image segmentation, and quantitative data processing to model the kinetics of reepithelialization of the gingival epithelial cell line Ca9-22 (JCRB0625). Using this assay, we screened 39 strains of LAB for their potential effects on re-epithelialization, hypothesising that certain $L A B$ strains produce specific compounds that can accelerate epithelial wound repair. The primary screening results were extended and confirmed in subsequent refined experiments that enabled the identification of Streptococcus salivarius MS-oral-D6 and its secreted protease as strong stimulators of gingival re-epithelialization. 


\section{Results}

\section{A novel strategy to extract and quantify the kinetics of the re-epithelialization process}

The throughput and reproducibility of the scratch assay was improved in this study by using a 96 -well system and the HTSScratcher, which consists of a 96 pin-array controlled by a counterforce mechanism that ensured that the downward movement of the array towards the 96 -well plate was conducted smoothly and consistently. Furthermore, we were able to quantify re-epithelialization over time by using automated microscopy in combination with image analysis. Images were acquired every 20 minutes until the scratches treated with human transforming growth factor a (hTGFa; positive control) were fully resolved. The tissue-recognition pipeline (Fig. 1a) of image analysis required a fully confluent cell monolayer to accurately determine the scratch size, but resembled the conventional way of analysing scratch assays in which the closure achieved with different treatments is compared after fixed periods of time ${ }^{103}$. The cell-recognition pipeline (Fig. 1b) enabled effective extraction of quantitative data by automatic identification of the number of cells in each image, which was accompanied by information regarding size, shape, stain-intensity, and location of each cell. The data acquired through this pipeline was visualized using the dedicated software FCS Express 4 Plus (De Novo Software, CA, USA) in which the number of cells infiltrating into the scratched area over time was determined for each well.

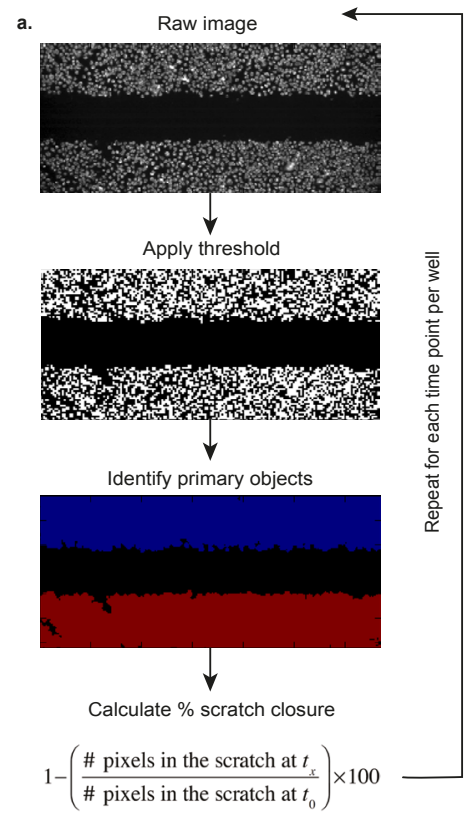

b.

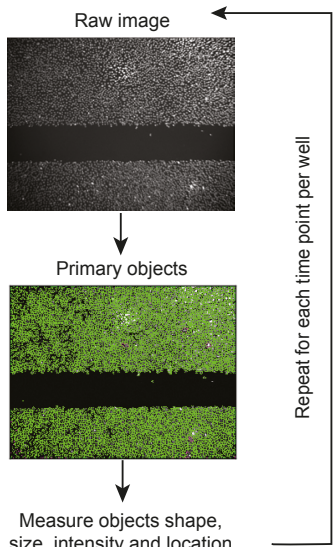

size, intensity and location
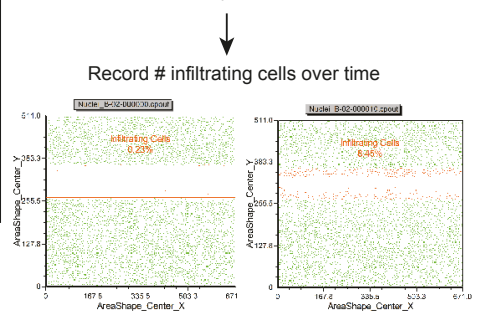

Figure 1. Automated image segmentation pipelines. (a) The tissue-recognition pipeline identifies the monolayer of cells as objects and the remaining pixels as the scratch. Thereafter, the percentage of scratch closure in consecutive images can then be calculated. (b) The cell-recognition pipeline identifies single cells in sequential images and records their location. A gate is manually set on the scratched area at time 0 and the number of epithelial cells infiltrating into the gate are recorded over time. 
The enumeration of infiltrating cells over time consistently resulted in a sigmoidal curve. Therefore, the modified Gompertz function (Fig. 2a), which has previously been used to model bacterial growth curves ${ }^{105}$, was fitted through the re-epithelialization measurements by a nonlinear least squares regression to estimate three biologically comprehensive parameters: $\mu_{\mathrm{m}^{\prime}} A$ and $\lambda$. The $\mu_{\mathrm{m}}$ parameter represents the repair rate (cells minute ${ }^{-1}$ ), and the A parameter represents the maximum number of cells within the scratched area at the plateau phase of the growth curve. The lag time or inflection point ( $\lambda$, described in minutes) represents the amount of time required for the cells to start moving into the scratched area and typically ranged from negative values to 20 minutes (Table S1). This implies that Ca9-22 cell- migration was already active when the image acquisition was initiated, and indicates that although the calculation of the $\lambda$ parameter is required to obtain a good fit of the model, its biological value was negligible. Thereby, the $\mu_{\mathrm{m}}$ and A parameters were sufficient to accurately describe the re-epithelialization kinetics with this cell line. Overall, the model yielded very good fits (Table S1; $R^{2}$ close to 1), illustrating that it effectively extracts quantitative re-epithelialization parameters from the serial images obtained and providing a high-throughput platform for quantification of wound repair kinetics. Moreover, the maximum number of cells (A parameter) obtained with the model was highly correlated with the relative scratch closure measured using the tissue-recognition pipeline $\left(r=0.678, P=1.36 \times 10^{-6}\right)$, which corroborated that enumeration of cells that infiltrated the scratched area provides a parameter that reflects the relative closure values obtained through the tissue-recognition pipeline (Fig. 2b). The workflow and crucial steps in the overall procedure are illustrated in Figure S1.
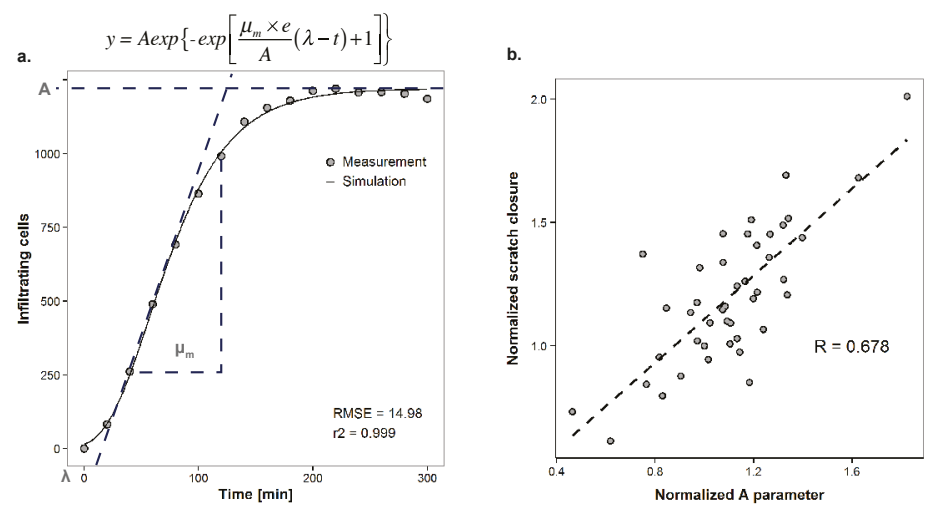

Figure 2. Modelling gingival re-epithelialization. (a) Representative sigmoidal curve obtained from the enumeration of epithelial cells infiltrating into the scratched area over time. Modified Gompertz function for sigmoidal curve fitting consisting of three parameters: the inflection point $(\lambda$, in minutes), the repair rate ( $\mu_{m^{\prime}}$ in cells minute ${ }^{-1}$ ) and the maximum number of cells ( $A$, in cells). (b) Spearman Rank Correlation coefficient to assess the relation between the A parameter and the relative scratch closure. All values were normalized against the non-treated control.

\section{Selection of potential stimulators and attenuators of gingival re-epithelialization}

The established scratch assay and downstream analysis method were used to screen a panel of 39 lactic acid bacteria (LAB) (Table 2) for their capacity to modulate the kinetics of gingival re-epithelialization. The initial screening was performed using duplicate measurements, and employing the $Z^{\prime}$ factor ${ }^{106}$ to assess the quality of individual assay runs on basis of the calculated dynamic range between of the positive and negative controls. Stimulation of the gingival cells with the hTGFa increased re-epithelialization kinetics more than 5-fold, and the combination of p38 and MEK1/2 inhibitors suppressed re-epithelialization kinetics approximately 2-fold, as compared to the non-treated control condition, respectively (Fig. 3). 
a.

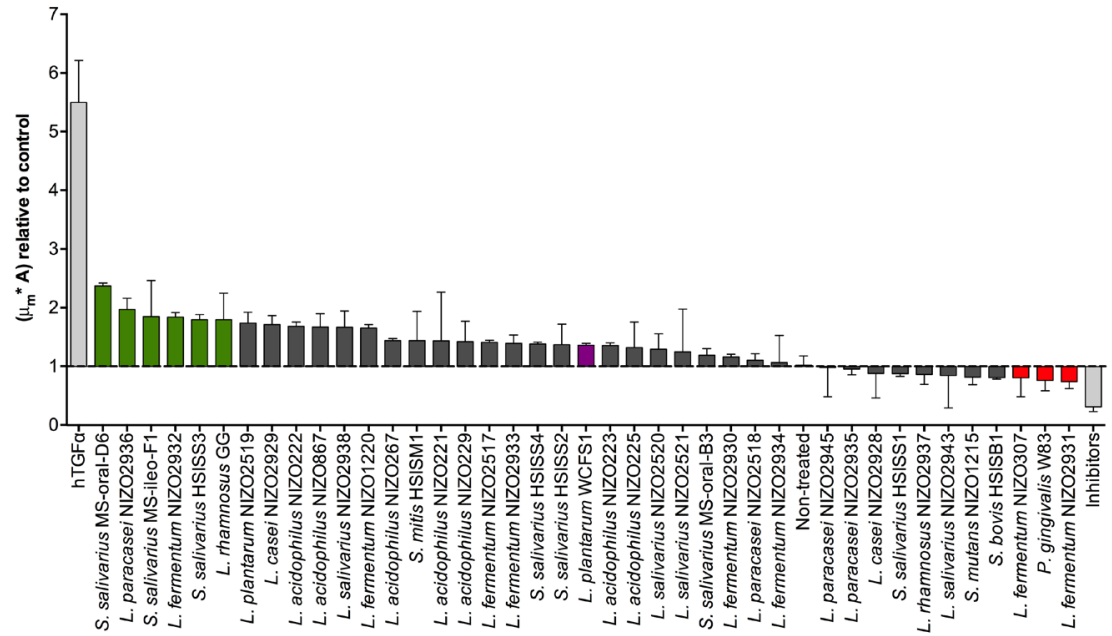

b.

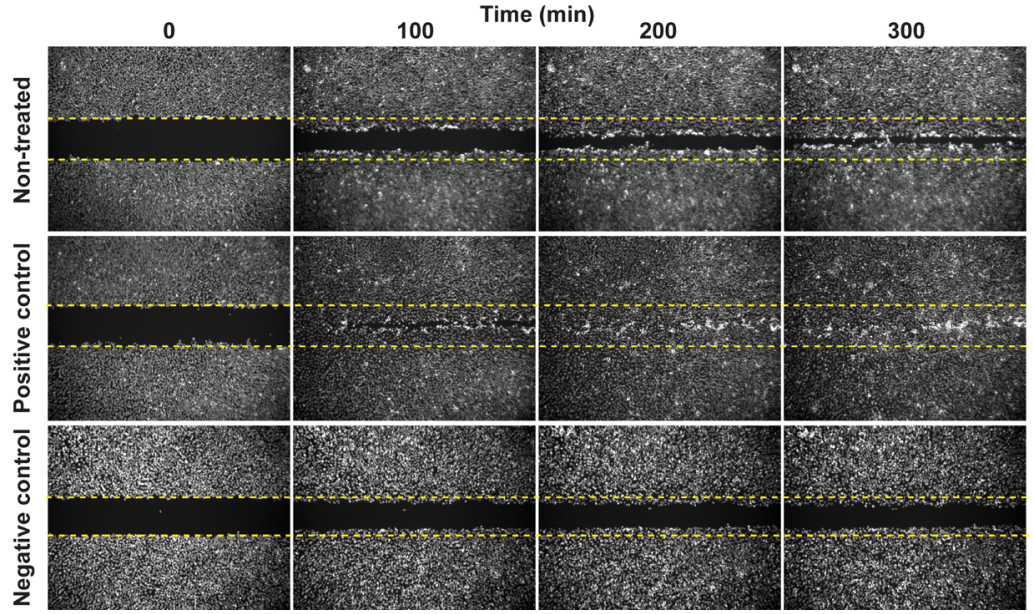

Figure 3. Modulatory effect of lactic acid bacteria in gingival re-epithelialization. (a) Overall performance of the bacterial treatments relative to the non-treated control $\left(\mu_{\mathrm{m}}{ }^{*} \mathrm{~A}\right)$. Results are expressed as mean \pm SEM. Green bars: potential stimulators; red bars: potential attenuators; purple bar: minor modulator. (b) Representative images for the non-treated, positive (hTGFa, $4 \mathrm{ng} / \mu \mathrm{l}$ ) and negative (p38 and MEK1/2 inhibitors, $10 \mu \mathrm{M}$ each) controls.

Most of the bacterial strains tested were originally isolated from the oral cavity. Other typical residents of the oral cavity (i.e. Streptococcus strains) were isolated from human ileostoma effluent, but were proposed by the authors ${ }^{107}$ to have originated from the oral cavity. In order to select potential stimulators and attenuators of re-epithelialization kinetics, a combinatorial approach was chosen that incorporated both relevant kinetic parameters from the measurements $\left(\mu_{\mathrm{m}}\right.$ and A) into a single performance value $\left(\mu_{m}{ }^{*} A\right)$ that was normalized against the non-treated control in each independent assay run (Fig. 3). Importantly, the cell-based assay and the automated pipelines of analysis clearly confirmed the previously reported stimulating effect of $L$. rhamnosus $\mathrm{GG}^{90,91,108}$ and the suppressive effect of $P$. gingivalis ${ }^{33,34}$ on re-epithelialization, which were both included in this study as reference strains for the establishment of the assay (Fig. 3; see also Fig. 
S2). Exposure of the gingival cells to $L A B$ at a multiplicity of infection (MOI) of 50 noticeably resulted in strain-specific modulatory effects of gingival re-epithelialization with some members of the same species having opposing effects (e.g. S. salivarius MS-oral-D6 and S. salivarius HSISS1; L. fermentum NIZO2932 and L. fermentum NIZO2931). Overall, the results showed a common tendency of $L A B$ to enhance the healing process, but none to a level that was comparable to the hTGFa-stimulated cells (Fig. 3a). The bacteria that elicited the strongest modulatory effects were selected for further studies. S. salivarius MS-oral-D6 and L. paracasei NIZ02936 were identified as the most prominent stimulators of gingival re-epithelialization, enhancing its kinetics by approximately 2.5 and 2-fold relative to the non-treated control, respectively. The selection of potential stimulators of gingival re-epithelialization was expanded with the next four strongest modulators of the process namely, S. salivarius MS-ileo-F1, L. fermentum NIZ02932, S. salivarius HSISS3 and L. rhamnosus GG (Fig. 3a). Furthermore, we were also capable of detecting potential suppressors of epithelial repair in comparison to the non-treated control. To this end, L. fermentum NIZO2931 and L. fermentum NIZ0307 were selected for further studies as they appeared to inhibit re-epithelialization to a level that was comparable to P. gingivalis W83. Finally, L. plantarum WCFS1, which is among the most intensely studied Lactobacillus strains, was selected as a representative of the strains that were more or less ineffective modulators of gingival re-epithelialization.

\section{Dose-response analysis confirms modulatory effects of selected bacteria on gingival re-epithelialization}

In order to confirm and refine the modulatory effects of the selected bacteria on gingival re-epithelialization, the scratch assay was performed using increasing dosages of bacterial preparations ( $\mathrm{MOI}$ of 10, 50 and 250) and a higher number of replicates $(n=3)$ in at least two independent experiments. Notably, all $S$. salivarius strains selected improved the re-epithelialization performance $\left(\mu_{m}{ }^{*} A\right)$ in a dose-dependent manner, resulting in a significant increase at the intermediate and higher dosages (S. salivarius MS-oral-D6 M0I $50(P=0.039)$ and $250(P<0.0001)$; S. salivarius MS-ileo-F1 MOI $50(P<0.0001)$ and $250(P<0.0001)$; S. salivarius HSISS3 MOI 50 $(P=0.001)$ and $250(P<0.0001))$ (Fig. 4a). Specifically, the S. salivarius strains accelerated the repair rate $\left(\mu_{\mathrm{m}}\right.$ parameter) in a dose dependent manner, leading to a higher number of cells (A parameter) in the scratched area as compared to the non-treated control (Fig. $4 \mathbf{C}, \mathbf{d}$ ). The promoting effect of $L$. rhamnosus $G G$ on the scratch resolution was also confirmed for the intermediate MOI tested ( $P=0.011$ ) (Fig. $4 \mathbf{a}, \mathbf{b}$ ), but it failed to significantly enhance the re-epithelialization kinetics at a higher dosage. Similarly, both the intermediate and higher dosages of L. paracasei NIZ02936 promoted reepithelialization by approximately 2 -fold and thus failed to show a clear dose-response relationship. Treatment with L. plantarum WCFS1 resulted in minor modulation of the re-epithelialization process regardless of the dosage used, confirming the lack of modulatory capacity of this strain in the re-epithelialization process as was concluded from the initial screening experiment (Fig. 4a, b). Exposure to increasing dosages of $P$. gingivalis W83 resulted in significant inhibition of re-epithelialization ( $P=0.037$ ), ultimately leading to actual deterioration of the cell monolayer (Fig. $4 \mathbf{a}, \mathbf{b}$ ). Finally, contrary to the initial screening results, stimulation of the epithelial cells with different dosages of the L. fermentum strains NIZO2931, NIZO2932 and NIZ0307 did not result in significant modulation of the healing process (Fig. 4a). 
a.
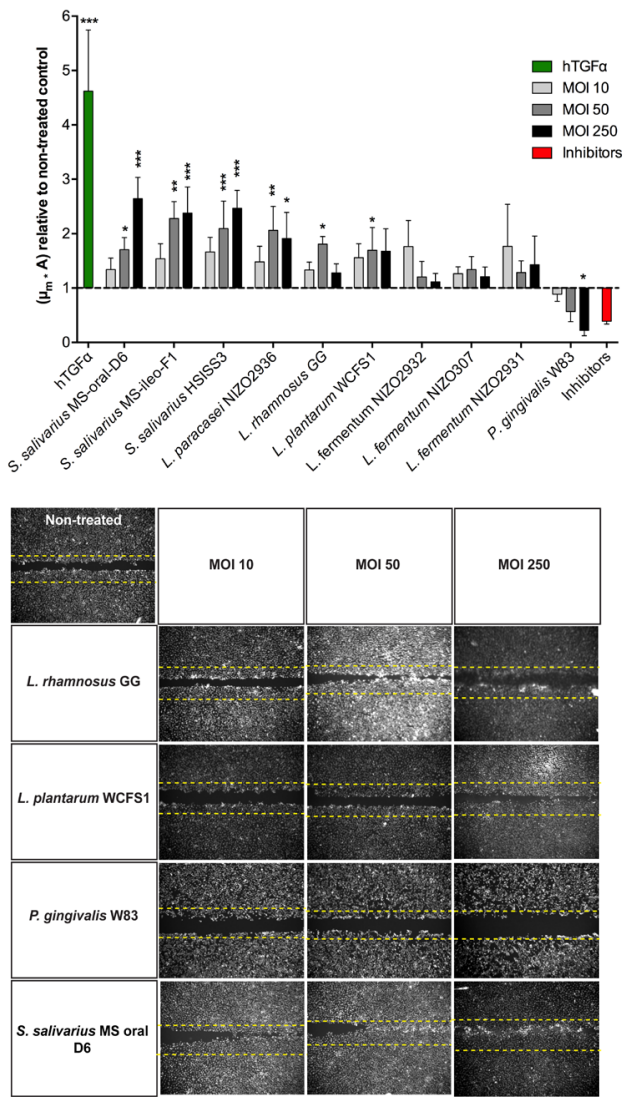

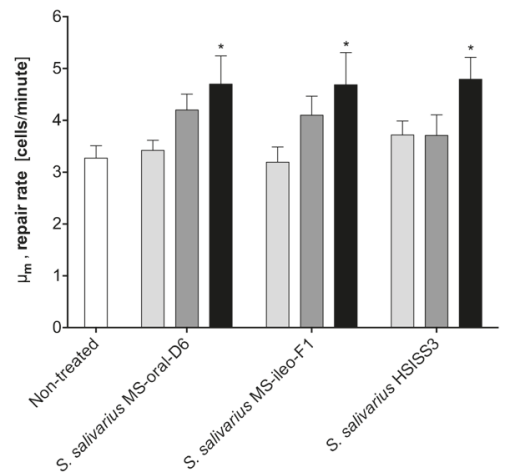

$\square$ MOI $10 \quad \square$ MOI 50

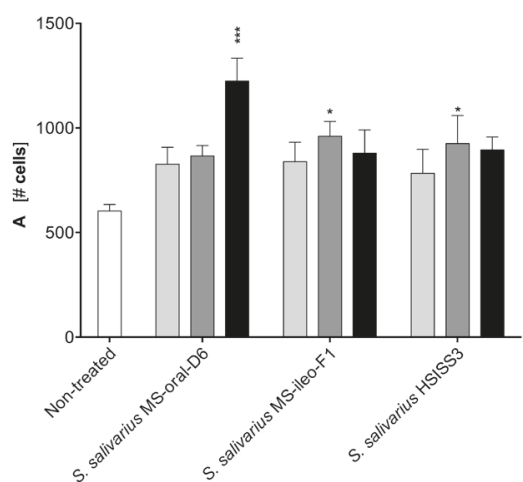

Figure 4. Dose response analysis of selected bacteria on gingival re-epithelialization. (a) The performance value $\left(\mu_{m}{ }^{*} A\right)$ of each treatment was calculated relative to the non-treated control. (b) Representative images of the re-epithelialization process after 5 hours exposure to bacteria at a multiplicity of infection (MOl) of 10, 50 and 250. (c) Repair rate obtained for the Streptococci strains. (d) Maximum number of cells in the scratched area obtained for the Streptococci strains. Results are expressed as mean \pm SEM from at least two independent experiments performed in triplicates. Significant differences from the non-treated control were assessed by a one-way ANOVA using a Dunnett's test for multiple comparisons $\left(^{*}\right.$, $P<0.05 ; * *, P<0.001 ; * *, P<0.0001)$.

\section{Influence of bacterial fermentation products and inflammatory capacity on re- epithelization}

To evaluate whether the modulatory effects of some bacteria on the kinetics of re-epithelialization were the result of the accumulation of fermentation end products, we quantified the concentration of lactic and acetic acid in the supernatants of the epithelial cell cultures 5 hours after addition of different dosages of bacteria (Table S2). The putative correlation between the detected concentrations of the lactic acid and acetic acid in individual wells with the kinetic parameter values $\left(\mu_{m}\right.$ and $\left.A\right)$ was subsequently evaluated by Pearson correlation analysis. A weak, but significant negative correlation ( $r=-0.279, P=0.04)$ between the acetic acid concentration and the A parameter was detected (Fig. S3). Notably, the concentrations of acetic acid in the supernatants of the cells exposed to the higher dosages of $L$. fermentum NIZ0307 and $P$. gingivalis W83, two of the strains with negative modulatory 
effect on re-epithelialization, were significantly higher than those of the non-treated control $(P<0.0001$ and $P=0.012$, respectively) (Fig. 5a). Conversely, significant positive correlations were detected between the lactic acid concentration and repair rate $\left(\mu_{\mathrm{m}} ; r=0.378, P=0.004\right)$ and the maximum number of cells $(A ; r=0.354$, $P=0.008$ ). The concentrations of lactic acid in the supernatants of cells exposed to $S$. salivarius MS-ileo-F1 (MOI 50, $P=0.019 ; \mathrm{MOI} 250, P<0.0001)$ and S. salivarius HSISS3 (MOI 50, $P<0.009)$ contained significantly more lactic acid than the non-treated control and were among the positive modulators of re-epithelialization kinetics. However, the strongest positive modulator of gingival re-epithelialization, S. salivarius MS-oral-D6 (Fig. 4), did not produce significant amounts of lactic acid during the assay, whereas L. plantarum WCFS1 produced substantial amounts of lactic acid (>3 mM) and failed to enhance the re-epithelialization process (Fig. $5 \mathbf{b}$ ), indicating that these bacteria can modulate the repair process via different mechanisms. Notably, exposure of the Ca9-22 cell line to L. plantarum WCFS1, but not to S. salivarius MSoral-D6 (both at MOI 250), elicited considerable production of the pro-inflammatory cytokine interleukin-8 (IL-8) (Fig. 5c), which may counteract the potentially stimulatory effect on re-epithelialization of the lactic acid produced by $L$. plantarum WCFS1.

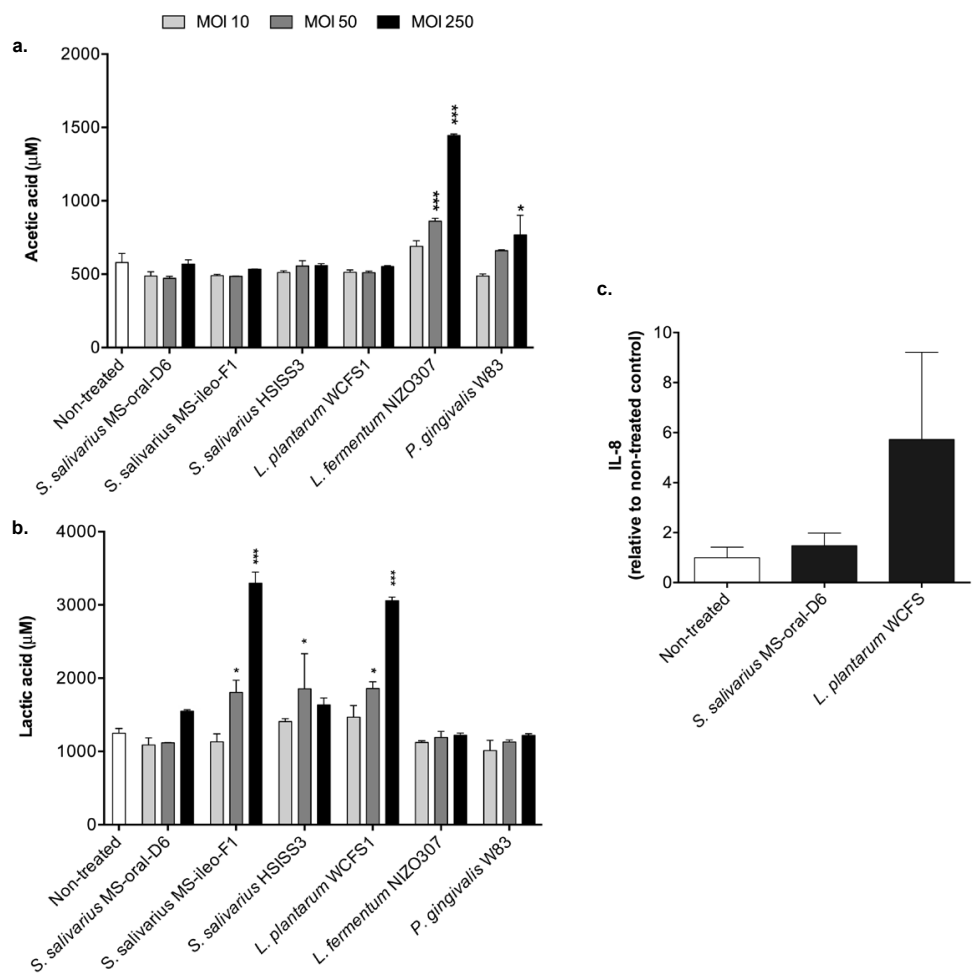

Figure 5. Fermentation end products of bacteria and gingival inflammatory response. (a) Acetic acid. (b) Lactic acid. The concentrations of these end-products of bacterial fermentation were measured in the supernatants of gingival cells stimulated with increasing MOls of bacteria after 5 hours. Results are expressed as mean \pm SEM from two independent experiments performed in triplicates. Significant differences from the non-treated control were assessed by a one-way ANOVA using a Dunnett's test for multiple comparisons ( ${ }^{*}$, $\mathrm{P}<0.05$; ${ }^{* * *}, \mathrm{P}<0.0001$ ). (c) Interleukin-8 (IL-8) produced by the gingival cells after 5 hours of exposure to the bacteria (M0I 250 ) expressed relative to the non-treated control. Results are expressed as mean \pm SEM from two independent experiments performed in duplicates. 


\section{The secreted serine protease of S. salivarius MS-oral-D6 accelerates gingival re- epithelialization}

To determine whether the stimulation of gingival re-epithelialization by S. salivarius strains was due to an extracellular molecule we tested spent culture supernatant (conditioned medium) in the scratch assay. The conditioned media of the $S$. salivarius strains tested were consistently accelerating re-epithelialization kinetics $\left(\mu_{m^{\prime}} A\right.$, and $\left.\mu_{m}{ }^{*} A\right)$ when compared with the original medium (i.e. TSB) (Fig. 6a), indicating that these bacteria may indeed secrete a component that is capable of promoting wound healing. To further investigate these effects, we removed small molecules and peptides from the conditioned medium using desalting columns with a $7 \mathrm{kDa}$ cut-off. The stimulatory effects of conditioned medium from $\mathrm{S}$. salivarius MS-ileo-F1 and HSISS3 on re-epithelialization were lost after passing the medium over a desalting column (Fig. $\mathbf{6 b})$, suggesting that the activity observed was due to the presence of small molecules $(<7 \mathrm{kDa})$, possibly involving lactic acid. In contrast, the conditioned medium of S. salivarius MS-oral-D6 retained its stimulatory effect on re-epithelialization after desalting, which was subsequently shown to be sensitive to proteinase $\mathrm{K}$ treatment $(200 \mu \mathrm{g} / \mathrm{ml})$, indicating that the activity is due to a secreted protein (Fig. $\mathbf{6 b}$ ). In addition, silver stained SDS-PAGE analysis of the desalted-conditioned medium of the $S$. salivarius strains revealed the presence of a high molecular weight (MW) protein (> $180 \mathrm{kDa}$ ) in the medium of S. salivarius MS-oral-D6 that was completely digested after proteinase $\mathrm{K}$ treatment (Fig. $6 \mathrm{C}$ ) and was absent from the media obtained from S. salivarius MS-ileo-F1 and HSISS3. In-gel trypsin digestion of the high MW protein followed by liquid chromatography tandem mass spectrometry (LC-MS/MS) and peptide mapping against the available $S$. salivarius genomes sequences (37 in total; Table S3) revealed the presence of 11 proteins from which the most abundant were identified as peptidoglycan hydrolase, serine protease, peptidase M26, and surface antigen (Fig. $\mathbf{6 c}$ and Table 1). From the identified proteins only the serine protease and the peptidase $M 26$ have a predicted MW that match the apparent MW of the band observed in the gel of $>180 \mathrm{kDa}$ (Table 1). Importantly, the gene encoding the serine protease appears to be encoded by only a fraction of the known S. salivarius genome sequences (12 out of 37), whereas the three other proteins appeared to be encoded by the vast majority of S. salivarius genomes (including the HSISS3 strain) (Table 1), which may support a role of the serine protease in the strain-specific stimulatory effect observed. Finally, to further investigate the possible involvement of the serine protease in enhanced re-epithelialization, the desalted-conditioned medium of S. salivarius MS-oral-D6 was pretreated with the serine protease inhibitor phenylmethane sulfonyl fluoride (PMSF, $1 \mathrm{mM}$ ) prior to being tested in the scratch assay. Following PMSF inactivation, addition of the MS-oral-D6 desaltedconditioned medium led to a reduction in re-epithelialization kinetics when compared to the control (Fig. 6c), supporting that the secreted serine protease of S. salivarius MS-oral-D6 is the effector molecule responsible for the enhanced reepithelialization kinetics observed.

Table 1. Proteins identified in the conditioned medium of S. salivarius MS-oral-D6. UniProt accession entries and gene IDs are provided with the predicted protein sizes $(\mathrm{kDa})$. The presence of the respective proteins in the sequenced genomes of $S$. salivarius is indicated as a percentage of the total number of genomes available $(n=37)$.

\begin{tabular}{lllll}
\hline Protein & Accession & Gene ID & Size (kDa) & Presence (\%) sequenced genomes \\
\hline Peptidoglycan hydrolase & A0A0A1DSA9 & SSAL8618_00185 & 56 & 92 \\
\hdashline Serine protease & A0A074J261 & DL07_03090 & 241 & 32 \\
\hdashline Peptidase M26 & A0A074IYQ3 & DL07_02275 & 222 & 81 \\
\hdashline Surface antigen & F8HFL8 & Ssal_01709 & 114 & 92 \\
\hline
\end{tabular}




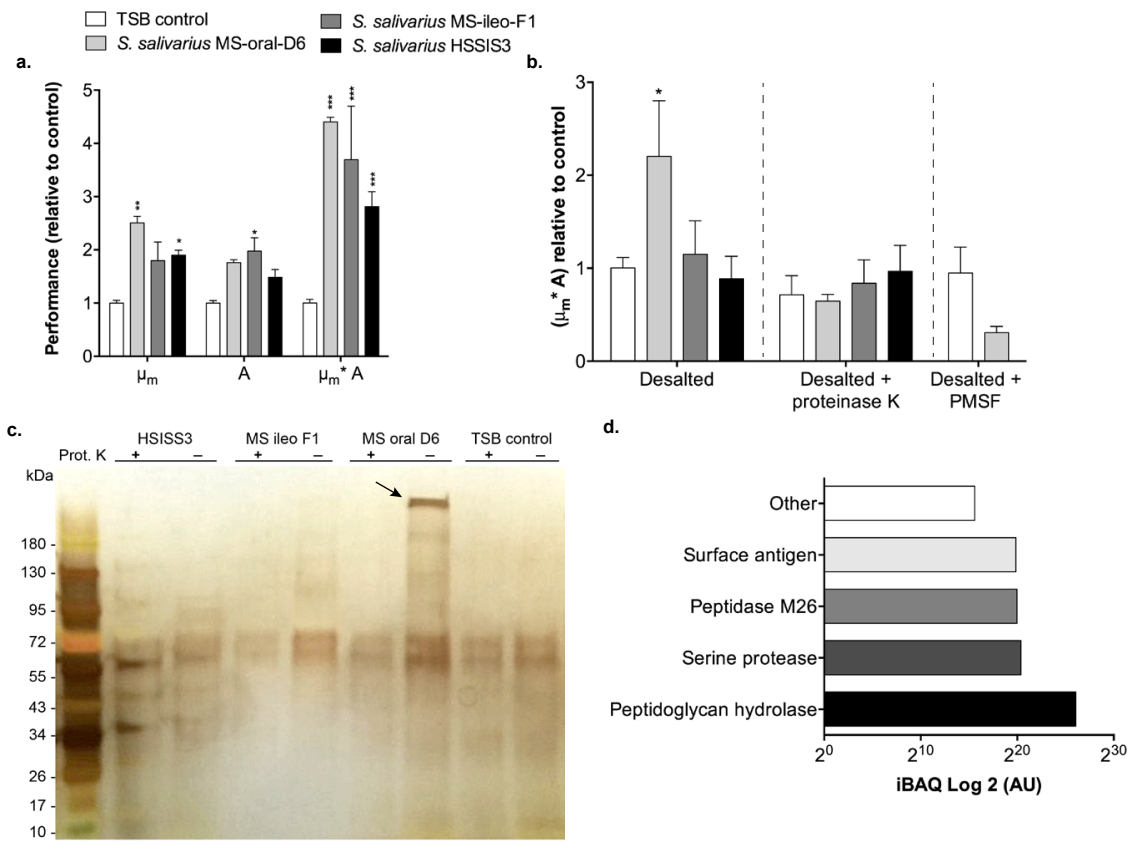

Figure 6. Evaluation of conditioned medium of Streptococcus salivarius strains on gingival re-epithelialization. (a) Re-epithelialization parameters $\left(\mu_{\mathrm{m}^{\prime}} A\right.$, and $\left.\mu_{\mathrm{m}}{ }^{*} \mathrm{~A}\right)$ of the $S$. salivarius conditioned medium relative to the TSB control. (b) Re-epithelialization performance values obtained with desalted-conditioned medium ( $7 \mathrm{kDa}$ cut-off) with or without proteinase $\mathrm{K}$ treatment $\left(200 \mu \mathrm{g} / \mathrm{ml}, 1\right.$ hour at $\left.37^{\circ} \mathrm{C}\right)$. S. salivarius MS-oral-D6 desalted-conditioned medium after PMSF treatment (1 mM, 1 hour at room temperature). Results are expressed as mean \pm SEM from two independent experiments performed in triplicates. Significant differences from the non-treated control were assessed by a one-way ANOVA using a Dunnett's test for multiple comparisons $\left({ }^{*}, P<0.05 ;{ }^{* *}, P<0.001 ;{ }^{* *}, P<0.0001\right)$. (c) Silver staining of the proteins present in desalted-conditioned medium before and after proteinase K (Prot. K) treatment. Arrow indicates the high MW band detected in S. salivarius MS-oral-D6 conditioned medium. (d) Proteins identified in the high MW band of the S. salivarius MS-oral-D6 conditioned medium. The bar labelled as "other" represents the sum abundance of seven other identified secreted proteins. Intensity based absolute quantitation (BAQ) refers to the total intensity corrected for the number of measurable peptides within a sample.

\section{Discussion}

Lactic acid bacteria $(L A B)$, in particular lactobacilli and streptococci, have been generally associated with caries ${ }^{109,110}$. Therefore, the idea of applying these microorganisms to promote oral health seems controversial. Nevertheless, there is increasing evidence implicating specific $L A B$ in oral health rather than disease. For instance, $L$. plantarum $299 \mathrm{v}, L$. reuteri (ATCC 55730 and ATCC PTA 5289) and L. rhamnosus GG have been shown to interfere with biofilm formation of S. mutans in vitro, which is still regarded as the primary etiological agent of dental caries ${ }^{111}$. Similarly, several isolates of L. paracasei and L. rhamnosus derived from healthy oral cavities, were shown to have antimicrobial activity against $S$. mutans and other oral pathogens including P. gingivalis and C. albicans ${ }^{97}$. Clinical interventions have provided evidence that $L$. reuteri ATCC 55730 can suppress cariogenic bacteria ${ }^{112-114}$ and improve periodontal health by diminishing gingival bleeding and reducing the levels of proinflammatory cytokines in subjects with moderate gingival inflammation ${ }^{115}$. In other studies, administration of $L$. salivarius WB21 or S. salivarius $\mathrm{K} 12$ resulted in improved periodontal health at the end 


\section{2 | Chapter 2}

of an intervention ${ }^{116,117}$ and suppression of volatile sulphur compounds (VSC)-producing bacteria, which are responsible for halitosis or "bad breath"99,117.

In this study, we evaluated the ability of oral LAB to promote gingival epithelial repair. The oral epithelium is the first line of defence against invading microorganisms and thus, upon disruption of barrier integrity, prompt re-epithelialization is essentia ${ }^{7,11}$. To study the effects of different $L A B$ on re-epithelialization, we developed a high-throughput scratch assay using live-automated fluorescence microscopy and image segmentation, which is coupled to a mathematical model that describes the kinetics of re-epithelialization. Contrary to traditional scratch assays, which are executed in 24-well plates and rely upon the manual introduction of a scratch ${ }^{103}$, we increased the throughput and reproducibility of the scratches by changing the assay format to 96-well plates and using the HTSScratcher (Peira) to generate uniform scratches. Previous studies ${ }^{118,119}$ have also been able to increase the throughput of the scratch assay by employing different scratching tools such as a 96-pin floating array and an 8-channel "wounder". However, these tools lacked the counterforce mechanism of the HTSScratcher, which ensures a smooth and consistent movement of the pin-array parallel to the wells. Consequently, HTSScratcher generates uniformly sized scratches with minimal damage to the plate coating, which could otherwise interfere with normal cell migration.

A key aspect of this high-throughput image-based method is the ability to automate the conversion of raw images into interpretable data. Hence, we established two analysis pipelines for image analysis in CellProfiler, one based on tissuerecognition and the other on cell-recognition. The tissue-recognition pipeline is a robust and straightforward analysis to determine the percentage of scratch closure through the image series of each well. Other open-source software such as ImageJ (https://imagej.nih.gov/ij/; "MRI Wound Healing Tool") and TScratch ${ }^{120}$ provide alternative options to carry out this type of analysis. Nevertheless, enumeration of single cells migrating into the scratched area over time provides quantitative data that can more readily be statistically analysed and enables the possibility of single cell tracking. Reepithelialization kinetics were described by biologically comprehensive parameters $\left(\mu_{m^{\prime}} A\right.$ and $\left.\lambda\right)$ derived from the modified Gompertz function ${ }^{105}$. The applied iterative optimization method yielded excellent fits and provided accurate estimation of the kinetics of the wound healing process.

Several studies have reported that Porphyromonas gingivalis attenuates re-epithelialization in scratch assays employing cell lines or primary gingival cells, and have proposed that this effect was at least partially dependent on the proteolytic activity secreted by this bacterial species ${ }^{79,80}$. Conversely, Lactobacillus rhamnosus $\mathrm{GG}$ has been shown to influence intestinal epithelial cells through the secretion of proteins (p75 and p40) that interact with the epidermal growth factor receptor (EGFR) to promote cell proliferation, differentiation, migration, and survival ${ }^{89,121}$. More recently, L. rhamnosus GG lysates were reported to promote re-epithelialization of skin-keratinocytes by increasing cell migration in vitro ${ }^{91}$, highlighting the possibility of expanding the applications of certain strains to promote beneficial effects in other bodysites such as the skin and the oral cavity. Hence, we included these microorganisms as benchmark bacteria to validate the assay developed in this study. The results obtained with these strains clearly confirm the attenuating and stimulating effects of $P$. gingivalis and $L$. rhamnosus $\mathrm{GG}$ in the $C$ a9-22 gingival re-epithelialization model, respectively.

The results of the preliminary screening revealed the common tendency of $L A B$ to stimulate re-epithelialization kinetics, although none of the strains tested stimulated to a level comparable to the positive control (hTGFa). The extent of the modulatory effect exerted by the bacteria could have been a result of the dosage selected for the screening (50 bacterial cells per epithelial cell; MOI 50) and/or the administration of viable bacteria. Interestingly, Mohammedsaeed et al. ${ }^{91}$ 
showed that L. rhamnosus GG lysates could promote skin-keratinocyte re-epithelialization to a level comparable to the keratinocyte growth factor used as positive control in that study. However, the lysate dosage employed in the study was very high ( $93 \mathrm{mg} /$ well) and exceeded the highest corresponding dosage of viable cells employed here by at least 1,000-fold. Dosages higher than the highest dose employed in this study (MOI 250) of viable L. rhamnosus GG (or many other $L A B$ ) are not feasible due to their capacity to acidify the cell culture medium during the assay, which interferes with cell viability. The initial screening revealed strain-specific effects on re-epithelialization by the different $L A B$, where $L$. fermentum NIZ02932 was identified as a potential stimulator, and L. fermentum NIZO2931 and NIZ0307 seemed to have the opposite effect. Nevertheless, upon exposure of the epithelial cells to increased dosages of these bacteria, a weakly negative effect on re-epithelialization was observed for all $L$. fermentum strains. Notably, high-dosages of lysates of $L$. fermentum ATCC 14932 (NIZO2930) were previously reported to significantly reduce skin-keratinocyte re-epithelialization in vitro ${ }^{91}$, but substantially lower dosages of viable cells of the same strain did not show modulatory effect in our model (Fig. 3a), which again may relate to the dosages used in the different studies.

Among the strongest and most consistent stimulators of oral re-epithelialization were the Streptococcus salivarius strains MS-oral-D6, MS-ileo-F1 and HSISS3. S. salivarius is one of the first bacterial species to colonize the intestinal and oral epithelial mucosa after birth ${ }^{122}$ and remains as a relatively dominant inhabitant of the oral cavity, upper respiratory tract and small intestine of healthy individuals throughout life ${ }^{37,123,124}$. Remarkably, co-culture of human bronchial epithelial cells with probiotic S. salivarius $\mathrm{K} 12$ altered the expression of 565 host genes involved in innate immunity pathways, epithelial cell function, cytoskeletal remodelling, cell development and migration ${ }^{100}$. Exposure of gingival epithelial cells to $S$. salivarius strains in this study resulted in acceleration of the repair rate in a dose-dependent manner. Although our results indicated that the re-epithelialization kinetics could be affected by the production of the main fermentation end products of these $L A B$ (i.e. acetic and lactic acid), these metabolites could not explain the stimulatory effects of the $S$. salivarius strains. In particular, S. salivarius MS-oral-D6 did not produce significant amounts of either of these metabolites and was the strongest stimulator of re-epithelialization. Previous in vivo wound-healing studies have shown that lactate accumulates in wounds $(4-12 \mathrm{mM}$ ) and regulates the healing process by stimulating the expression of the vascular endothelial growth factor (VEGF) and collagen deposition ${ }^{125,126}$. However, the concentrations of lactic acid measured after 5 hours in the culture media that contained LAB were in most cases lower than $4 \mathrm{mM}$. An exception was the cells exposed to the highest dosage of $L$. paracasei NIZO2936, where lactic acid concentrations increased to final concentrations of up to $5 \mathrm{mM}$ (Table S2) and only moderate stimulation of re-epithelialization kinetics was observed. These findings suggest that the contribution of the lactic acid to the wound-healing process in our study was limited, and indeed concentrations up to $6 \mathrm{mM}$ of D- or L-lactic acid at neutral pH did not affect the re-epithelialization kinetics in our model (Fig. S4), implying that other components play an important role in the observed modulations of re-epithelialization kinetics. Among such other components, may be those that trigger pro-inflammatory responses in epithelial cells, which may delay wound repair by blocking the essential transition from the inflammatory to the proliferative phase of wound healing ${ }^{127,128}$. Measurement of IL-8, a pro-inflammatory cytokine that attracts and activate neutrophils and acts as a mediator of angiogenesis ${ }^{129}$, showed that treatment with S. salivarius MS-oral-D6 did not result in increased inflammation, which was in clear contrast to $L$. plantarum WCFS1 and may be involved in the inability of the latter bacteria to significantly modulate re-epithelialization.

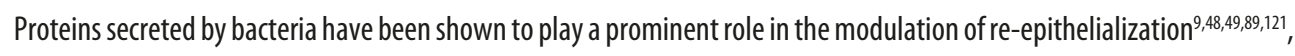
and we could show that filter-sterilized conditioned medium of three $S$. salivarius strains used in this study consistently accelerated re-epithelialization. However, this effect appeared to depend on a larger sized (> $7 \mathrm{kDa}$ ) secreted protein only 
present in the spent medium obtained from S. salivarius MS-oral-D6. Correspondingly, a high MW protein (> $180 \mathrm{kDa}$ ) was only detected in the conditioned medium of S. salivarius MS-oral-D6 and not in that of strains MS-ileo-F1 and HSISS3. Proteomic analysis suggested that this protein could correspond to a secreted serine protease based on its apparent molecular weight as well as the diversity of the presence of its encoding gene in different strains of $S$. salivarius. Moreover, the involvement of this serine protease as the effector molecule in the observed stimulation of re-epithelialization kinetics is supported by the abolishment of the effect on wound healing by serine protease inhibitor (PMSF) pretreatment. The secreted serine protease of $\mathrm{S}$. salivarius is a member of the subtilases superfamily characterized by a catalytic triad (Asp/ $\mathrm{Ser} / \mathrm{His}$ ) similar to the one in trypsine serine protease ${ }^{130}$. Notably, protease activity is essential for normal wound healing and is known to play a role in different phases of healing, including aiding detachment and migration of cells ${ }^{131}$. Taken together, the presence of the serine protease in the conditioned medium of S. salivarius MS-oral-D6 may explain the strain specific effects observed on re-epithelialization. However, a role of additional proteins, which may include the other proteins identified in the same strain's conditioned medium cannot be excluded at this stage.

In conclusion, this study presents a high-throughput re-epithelialization model that enables the extraction of kinetic information regarding the capacity of bioactive substances to modulate this process. The model developed here uses the gingival cell line (a9-22, but it is applicable to other cell lines as well (data not shown). Screening LAB for their effect in re-epithelialization led to the identification of $S$. salivarius as one of the most stimulatory species among the panel of organisms tested. In addition, S. salivarius MS-oral-D6 secreted serine proteases was identified as the most likely candidate effector molecule involved in enhanced re-epithelialization. It remains to be determined by what molecular mechanism this protein could modulate epithelial cell processes, including the acceleration of wound closure. Moreover, the possible contribution of other proteins produced and secreted by S. salivarius MS-oral-D6 to the accelerated reepithelialization is currently not known and also deserves further study. Such mechanistic studies may open avenues to identify S. salivarius strains that produce the effector protein(s) in high amounts and could be considered as probiotics to support epithelial integrity in the oral cavity, or may enable strategies that involve purified forms of such protein(s) as a 'bioactive' in treatments with similar purposes. This study illustrates the importance of identification and mechanistic understanding of the involvement of microbial components in maintenance of oral health which can fuel innovative strategies to support oral health.

\section{Materials and Methods}

\section{Epithelial cell line}

Ca9-22 (JCRB0625) gingival epithelial cells were purchased from the National Institute of Biomedical Innovation JCRB Cell Bank (Osaka, JP). The cells were cultured in Dulbecco's Modified Eagle Medium (DMEM) containing Glutamax (Gibco, Invitrogen, Paisley, UK) and supplemented with $10 \%$ fetal calf serum (FCS), $100 \mathrm{U} / \mathrm{ml}$ penicillin and $100 \mu \mathrm{g} / \mathrm{ml}$ streptomycin (Sigma-Aldrich, MO, USA). The cell line was cultured at $37^{\circ} \mathrm{C}$ in a humidified atmosphere containing $5 \% \mathrm{CO}_{2}$ and passaged every three to four days. Experiments were carried out between passages three to twenty. 


\section{Bacterial strains and culture}

The lactic acid bacteria (LAB) used in this study are listed in Table 2. The Lactobacillus strains and Streptococcus mutans NIZ01215 were obtained from the NIZO culture collection (NIZO food research, Ede, NL). The other streptococci were kindly provided by Dr. Tom van den Bogert from the Laboratory of Microbiology of the Wageningen University (Wageningen, NL). Lactobacillus strains were routinely cultured in de Man, Rogosa and Sharpe (MRS) medium (Merck Millipore, Darmstadt, DE) and Streptococcus strains were cultured in trypticase soy broth (TSB) (BD Difco, Le Pont de Claix, FR) supplemented with yeast extract $(3 \mathrm{~g} / \mathrm{L})$. All lactic acid strains were grown at $37^{\circ} \mathrm{C}$ with $5 \% \mathrm{CO}_{2}$. Porphyromonas gingivalis W83 was kindly provided by Dr. Alexa M. G. A. Laheij from ACTA (Academic Centre for Dentistry Amsterdam, Amsterdam, NL) and grown anaerobically at $37^{\circ} \mathrm{C}$ in Brain Heart Infusion (BHI) broth (BD Difco, Le Pont de Claix, FR) supplemented with haemin $\left(5 \mathrm{mg} / \mathrm{L}\right.$ ) and menadione (1 mg/L). The GasPak ${ }^{\text {TM }}$ EZ Anaerobe Container System Sachets (Becton, Dickinson and Company, NJ, USA) were used to produce an anaerobic environment inside of the containers.

Table 2. Lactic acid bacteria used in the screenings for their effect in oral re-epithelialization.

\begin{tabular}{|c|c|c|c|}
\hline$\#$ & Species identification & Origin & Reference \\
\hline 1 & Lactobacillus acidophilus NIZO221 & Human & 95 \\
\hline 2 & Lactobacillus acidophilus NIZO222 & N/A & 95 \\
\hline 3 & Lactobacillus acidophilus NIZO223 & N/A & 95 \\
\hline 4 & Lactobacillus acidophilus NIZO225 & N/A & 95 \\
\hline 5 & Lactobacillus acidophilus NIZO229 & N/A & 95 \\
\hline 6 & Lactobacillus acidophilus NIZO267 & N/A & 95 \\
\hline 7 & Lactobacillus acidophilus NIZ0867 & Human & 95 \\
\hline 8 & Lactobacillus casei NIZO2928 & Human saliva & 95 \\
\hline 9 & Lactobacillus casei NIZO2929 & Human saliva & 95 \\
\hline 10 & Lactobacillus fermentum NIZ01220 & N/A & 95 \\
\hline 11 & Lactobacillus fermentum NIZO2517 & Human oral cavity & 95 \\
\hline 12 & Lactobacillus fermentum NIZO2930 & Saliva & 95 \\
\hline 13 & Lactobacillus fermentum NIZO2931 & Human oral strain & 95 \\
\hline 14 & Lactobacillus fermentum NIZO2932 & Human saliva & 95 \\
\hline 15 & Lactobacillus fermentum NIZO2933 & Human saliva & 95 \\
\hline 16 & Lactobacillus fermentum NIZ02934 & Human saliva & 95 \\
\hline 17 & Lactobacillus fermentum NIZ0307 & Human oral cavity & 95 \\
\hline 18 & Lactobacillus paracasei NIZO2518 & Child saliva & 95 \\
\hline 19 & Lactobacillus paracasei NIZO2935 & Oral source & 95 \\
\hline 20 & Lactobacillus paracasei NIZ02936 & Child saliva & 95 \\
\hline 21 & Lactobacillus paracasei NIZO2945 & Oral cavity & 95 \\
\hline 22 & Lactobacillus plantarum NIZO2519 & Human saliva & 95 \\
\hline 23 & Lactobacillus plantarum WCFS1 & Human saliva & 132 \\
\hline 24 & Lactobacillus rhamnosus $\mathrm{GG}^{*}$ & Human intestine & 133 \\
\hline 25 & Lactobacillus rhamnosus NIZO2937 & Human saliva & 134 \\
\hline 26 & Lactobacillus salivarius NIZO2520 & Saliva & 95 \\
\hline 27 & Lactobacillus salivarius NIZO2521 & Saliva & 95 \\
\hline 28 & Lactobacillus salivarius NIZO2938 & Human saliva & 95 \\
\hline 29 & Lactobacillus salivarius NIZO2943 & Human saliva & 95 \\
\hline 30 & Porphyromonas gingivalis W83* & Human gingival sulcus & 79 \\
\hline 31 & Streptococcus bovis HSISB1 & Human ileostomy & 135 \\
\hline 32 & Streptococcus mitis HSISM1 & Human ileostomy & 135 \\
\hline 33 & Streptococcus mutans NIZ01215 & N/A & 95 \\
\hline 34 & Streptococcus salivarius HSISS1 & Human ileostomy & 135 \\
\hline 35 & Streptococcus salivarius HSISS2 & Human ileostomy & 135 \\
\hline 36 & Streptococcus salivarius HSISS3 & Human ileostomy & 135 \\
\hline 37 & Streptococcus salivarius HSISS4 & Human ileostomy & 135 \\
\hline 38 & Streptococcus salivarius MS-ileo-F1 & Human ileostomy & 135 \\
\hline 39 & Streptococcus salivarius MS-oral-B3 & Human oral cavity & 135 \\
\hline 40 & Streptococcus salivarius MS-oral-D6 & Human oral cavity & 135 \\
\hline
\end{tabular}

*P. gingivalis and L. rhamnosus GG were included in this study for reference purposes. N/A not assigned. 


\section{Preparation of bacterial treatments}

Bacterial strains were grown until stationary phase, after which glycerol 15\% (v/v) stocks were prepared and stored at $-80^{\circ} \mathrm{C}$ until used. $0 \mathrm{n}$ the day of the experiment, glycerol stocks were thawed and bacteria were pelleted by centrifugation $(4,000 \times \mathrm{g}, 7$ minutes at room temperature). The spent media were removed and the bacteria were re-suspended in FCSfree DMEM to obtain the original optical density at $600 \mathrm{~nm}(0 \mathrm{D} 600)$. The samples were further diluted in FCS-free DMEM until the required multiplicity of infection (MOI). An $0 D 600$ of 1 corresponded to approximately $5 \times 10^{8} \mathrm{CFU} / \mathrm{ml}$. Scratch assays were performed using different MOIs. Initial screening efforts employed a standard $\mathrm{MO}$ of 50 , whereas subsequent experiments employed MOIs of 10,50 and 250 to obtain dose-response curves.

\section{Conditioned medium collection and preparation}

Bacterial strains were grown until stationary phase. Subsequently, the bacterial cells were removed by centrifugation $(4,000 \times \mathrm{g}, 7$ minutes at room temperature) to obtain the spent culture medium (conditioned medium) of which the $\mathrm{pH}$ was adjusted to 7.0 by addition of $1 \mathrm{M} \mathrm{NaOH}$. Conditioned medium samples were filter-sterilized using a syringe filter with a pore size of $0.2 \mu \mathrm{m}$ (Whatman, GE Healthcare Life Sciences, Dassel, DE) and tested for their capacity to promote re-epithelialization in comparison with the control medium (i.e. TSB medium in absence of bacteria). In order to remove small molecules and/or peptides as well as other bacterial growth medium constituents, the conditioned medium and control medium were passed through Zeba ${ }^{\mathrm{TM}}$ Spin Desalting columns (7 kDa cutoff, Thermo Scientific, Rockford, IL, USA). The desalted samples were divided in two equal volume aliquots, one of which was treated with $200 \mu \mathrm{g} / \mathrm{ml}$ proteinase $\mathrm{K}$ $\left(1\right.$ hour at $37^{\circ} \mathrm{C}$ ) and the other was left untreated. Inactivation of proteinase $\mathrm{K}$ was accomplished by heating the samples to $98^{\circ} \mathrm{C}$ for 10 minutes, followed by centrifugation ( $16,000 \times \mathrm{g}$, $10 \mathrm{~min}$ at room temperature) $)^{136}$. After centrifugation, all samples were filtered sterilized $\left(0.2 \mu \mathrm{m}\right.$, Whatman, GE Healthcare Life Sciences) and then stored at $-80^{\circ} \mathrm{C}$ until used. Desalted-conditioned medium derived from S. salivarius MS-oral-D6 were treated with $1 \mathrm{mM}$ phenylmethane sulfonyl fluoride (PMSF) for 1 hour at room temperature in order to inactivate the serine proteases present in the samples. After inactivation, the samples were stored at $-80^{\circ} \mathrm{C}$ until used.

\section{SDS-PAGE and silver staining}

Conditioned medium proteins were precipitated with $10 \%(\mathrm{w} / \mathrm{v})$ iced-cold trichloroacetic acid (TCA) in a 1:1 ratio and incubated for 30 minutes on ice. After incubation, the samples were centrifuged at $16,000 \mathrm{x} \mathrm{g}$ for 10 minutes at $4^{\circ} \mathrm{C}$. The supernatant was discarded and the protein pellet was washed twice with ice-cold acetone. The acetone was removed and the pellet was air-dried. Precipitated proteins were taken up in $2 \mathrm{X}$ Laemmli sample buffer ${ }^{137}$ with $1 \% \beta$-mercaptoethanol and after boiling the samples, they were analysed by sodium dodecyl sulfate polyacrylamide gel electrophoresis (SDSPAGE) using 4-15\% Mini-POTEAN ${ }^{\circledR}$ TGX ${ }^{\mathrm{TM}}$ Precast Gels (Bio-Rad Laboratories, CA, USA). The SilverXpress ${ }^{\circledR}$ Silver Staining Kit (Thermo Scientific) was used to visualize the proteins in gel.

\section{Scratch Assay}

Cells were seeded in tissue culture-treated 96-well plates (BD Falcon ${ }^{\mathrm{TM}}$, Corning, NY, USA) at a density of $3.5 \times 10^{4}$ cells/ well and grown overnight until confluence in DMEM supplemented with $10 \% \mathrm{FCS}$. The next day, cells were starved in FCSfree DMEM for 2 hours in order to decrease the basal levels of cell migration and proliferation that is induced by growth 
stimulatory components present in FCS. During the last 20 minutes of the starvation, the cellular cytoplasm were labelled with $2 \mu$ M CellTracker ${ }^{\text {TM }}$ Red CMTPX (Molecular Probes, OR, USA) and the nuclei were stained with $2 \mu \mathrm{g} / \mathrm{ml}$ Hoechst 33342 (Molecular Probes). Following 2 hours starvation, the HTSScratcher (Peira, Antwerpen, BE) was used to make equally sized and reproducible longitudinal scratches $(0.3 \times 2 \mathrm{~mm})$ in the cell monolayers of all the wells simultaneously. Detached cells and debris were removed by washing the cells twice with phosphate buffered saline (PBS). Subsequently, $100 \mu$ l of the bacterial treatments and controls in DMEM without FCS were added into the wells in a randomized manner. Conditioned medium was diluted in FCS-free DMEM in a 1:2 ratio. The positive control consisted of $4 \mathrm{ng} / \mathrm{ml}$ human transforming growth factor a (TGFa; R\&D Systems, MN, USA), which acts as a mitogenic and motility factor. A combination of inhibitors of p38 (SB203580; Cell Signaling Technology, MA, USA) and MEK1/2 (U0126, Cell Signaling Technology) at a concentration of $10 \mu \mathrm{M}$ each, served as negative control. Inhibition of $\mathrm{p} 38$ mitogen-activated protein kinase (MAPK) phosphorylation led to suppression of cell migration ${ }^{138,139}$ and inhibition of MEK1/2 phosphorylation blocked the activation of ERK1/2 MAPK cascade that mediates cell proliferation and survival ${ }^{140}$. FCS-free DMEM was used as non-treated control. The overall quality of each run of the 96-well based assay was assessed by calculation of the $Z^{\prime}$ factor, which is defined by the means and standard deviations of both the positive and negative controls. Values between 0.5 and 1 indicated an excellent assay; values between 0 and 0.5 were considered acceptable; and assays with negative values were discarded ${ }^{106}$.

\section{Automated image acquisition and segmentation}

Live microscopy images were acquired using the BD Pathway 855 Bioimaging System (BD Biosciences, CA, USA) under controlled temperature and atmosphere $\left(37^{\circ} \mathrm{C}\right.$ and $\left.5 \% \mathrm{CO}_{2}\right)$. Hoechst 33342 and CellTracker ${ }^{\mathrm{TM}}$ Red CMTPX images were acquired using an excitation filter of $350 \mathrm{~nm}$ and $577 \mathrm{~nm}$, respectively. Bright-field images served as visual control for cell morphology and vitality. The microscope was programmed to acquire fluorescent and bright-field images of the same field of each well every 20 minutes for 5 hours using a $4 x$ objective. Image segmentation was carried out using CellProfiler 2.1.1 (http://www.cellprofiler.org/). Two segmentation pipelines were established: a tissue-recognition pipeline and a cell-recognition pipeline. The tissue-recognition pipeline was designed to identify the number of pixels of the scratched area that were covered by the monolayer of cells (tissue) in consecutive images. The number of pixels constituting the scratch was calculated by subtracting the pixels covered by cells from the total number of pixels in the image. The percentage of scratch closure in consecutive images was then calculated as 1 - [(\# pixels in the scratch at time $\mathrm{x}$ )/(\# pixels in the scratch at time 0$) * 100]$. The cell-recognition pipeline was designed to identify single cells in sequential images and record their location as well as other features such as shape, size and fluorochrome intensity. The features extracted by the cell-recognition pipeline were then used to model the kinetics of the re-epithelialization process.

\section{Modelling of the kinetics of oral re-epithelialization}

FCS Express 4 Plus (De Novo Software, CA, USA) was used to visualize the features extracted by the cell-recognition pipeline established in CellProfiler. A scatterplot was created with the location $(x, y)$ of the cells within a well at the starting point of the assay. A gate was placed on the scratched area and a batch process was setup for each well to record the number of cells infiltrating the wound throughout time. The lag time ( $\lambda$ in minutes), repair rate $\left(\mu_{m}\right.$ in cells minute- 1$)$ and the maximum number of cells (A) inside of the scratched area of each well were calculated in $R$ (http://www.R-project.org/ ${ }^{141}$ by a nonlinear least squares regression of the modified Gompertz function ${ }^{105}$ using the Levenberg-Marquardt algorithm ${ }^{142}$. 
The Levenberg-Marquardt algorithm reduces the sum of the squared errors between the modelled and the measured data points by iteratively improving the parameter values ${ }^{143}$. The $\mu_{\mathrm{m}}$ and A parameter values of each treatment were normalized against the corresponding average values of the non-treated control to obtain the performance value $\left(\mu_{m}{ }^{*} A\right)$. When appropriate (i.e. $n \geq 3$ ), replicate averages and standard error of the mean (SEM) for each treatment were calculated and significant differences from the non-treated control were determined using GraphPad Prism version 6.00 (GraphPad Software, Inc, (A, USA) with a limit of significance set at $P<0.05$. To assess the relation between the $A$ parameter and the relative of scratch closure, average values were normalized against the non-treated control and their relation was assessed in R using Spearman Rank Correlation.

\section{Quantification of lactate, acetate and IL-8 in cell culture supernatants}

Fermentation end products (i.e. lactic acid and acetic acid) of $L A B$ during the 5 hours re-epithelialization assay were determined in cell culture supernatant collected at the end of the 5 hours re-epithelialization assay by nuclear magnetic resonance (NMR), using an internal standard $(0.5 \mathrm{mM})$ to calculate the absolute concentrations of both acids in each sample. The potential correlation of the metabolite concentrations with the observed wound repair kinetics observed in the re-epithelialization assay was assessed in R using Pearson Correlation analysis. The ELISA Ready SET Go! Kit (BD Biosciences, (A, USA) was used to determine the amount of interleukin-8 (IL-8) in the supernatants of cells exposed to selected bacterial treatments ( $M 0 \mathrm{I} 250$ ) during the 5 hours re-epithelialization assay.

\section{In-gel trypsin digestion and LC-MS/MS}

The proteins contained in the conditioned medium of Streptococcus salivarius MS-oral-D6 were precipitated with 10\% (w/v) iced-cold trichloroacetic acid (TCA) and separated by SDS-PAGE as described in Materials and Methods. Readyto-use Coomassie protein stain InstantBlue (Expedeon, Cambridgeshire, UK) was used to visualize the proteins on gel. In-gel trypsin digestion followed by liquid-chromatography tandem mass spectrometry (LC-MS/MS) was performed as previously described ${ }^{144}$. The protein band ( $>180 \mathrm{kDa}$ ) was excised from the gel and transferred to a low binding protein microcentrifuge tube. A blank gel slice was used as control. The gel pieces were washed twice with water and disulfide bridges were then reduced with $10 \mathrm{mM}$ dithiothreitol (DTT) in $50 \mathrm{mM}$ ammonium bicarbonate ( $A B C$, pH 8.0) for one hour at $60^{\circ} \mathrm{C}$. Subsequently, the DTT is replaced by $15 \mathrm{mM}$ iodoacetamide in in $50 \mathrm{mM}$ Tris buffer $\mathrm{pH} 8.0$ and incubated at room temperature in the dark for one hour. After washing twice with $A B C$, the gel pieces were incubated overnight at room temperature in $\mathrm{ABC}$ containing $5 \mathrm{ng} / \mu \mathrm{l}$ bovine sequencing grade trypsin (Roche). The samples were sonicated briefly in an ultrasonic water bath to extract the digested peptides after which $10 \%$ trifluoroacetic acid (TFA) was added to adjust the pH between 2 and 4. To assure gel-free samples, the peptide solutions were desalted using C18 stage-tip columns made in-house. The columns were washed twice with methanol and equilibrated with formic acid (1:1000). Peptide solutions were added to the columns and eluted with a vacuum manifold. The columns were then washed with formic acid and transferred to low binding protein microcentrifuge tubes. Peptides were eluted with solution of $50 \%$ acetonitrile and $50 \%$ formic acid (1:1000) using a 10-ml plastic syringe. The LC-MS/MS analysis was performed on a Proxeon EASY-nLC system (Thermo Scientific) coupled with an LTQ-Orbitrap XL mass spectrometer (Thermo Scientific). For protein identification and relative quantitation, MaxQuant 1.3.0.5 software package was used with false discovery rates of $1 \%$ both on peptide and protein level after matching the MS/MS spectra against a common contaminants database and S. salivarius database. 


\section{Acknowledgements}

We thank Koos Oosterhaven and Zeger Kruiswijk (NIZO Food Research, Ede, NL) for providing us with a collection of lactic acid bacteria derived from the oral cavity. We also want to acknowledge Dr. Alexa M. G. A. Laheij (Academic Centre for Dentistry Amsterdam, Amsterdam, NL) for sharing her experience in re-epithelialization assays and providing $P$. gingivalis W83, as well as Dr. Tom van den Bogert (Laboratory of Microbiology, Wageningen University, Wageningen, $\mathrm{NL}$ ) for providing us with the Streptococcus strains. Finally, we are grateful for the advice and expertise provided by Sjef Boeren and Berdien van Olst (Biochemistry group, Wageningen University, Wageningen, $\mathrm{NL}$ ) regarding the execution and interpretation of proteomics data. Finally, we thank Lisa Robbers for her help executing cytokine measurements. 


\section{Supplementary data}

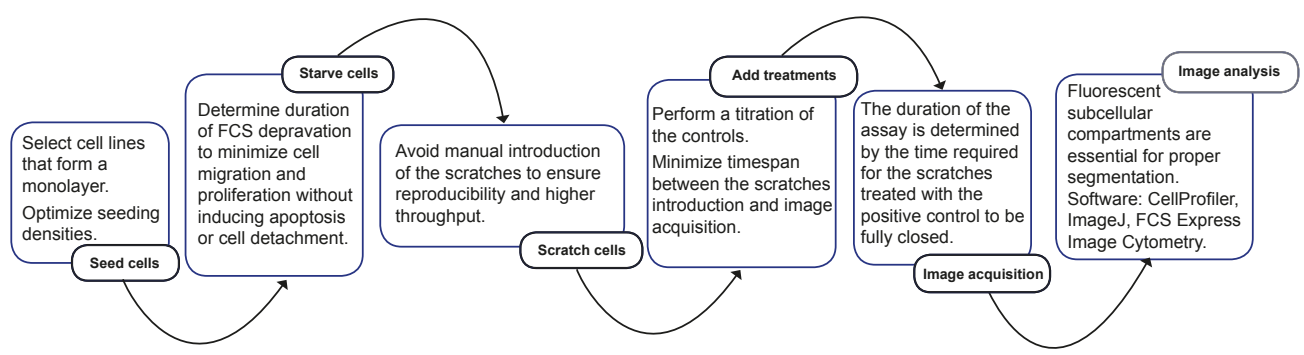

Figure S1. Scratch assay workflow. The assay workflow and crucial considerations are indicated in each step.

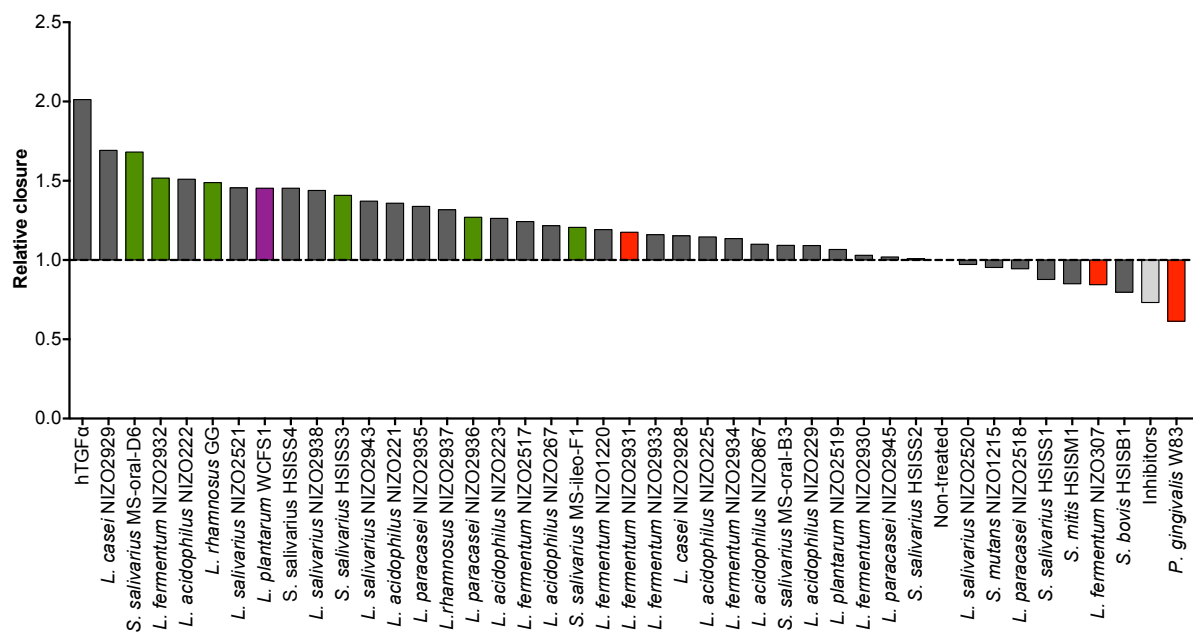

Figure S2. Relative scratch closure after exposure to different bacterial treatments. The closure of the scratches after stimulation of Ca922 cells with the bacterial treatments was calculated relative to that of the non-treated control. Green bars: potential stimulators; red bars: potential attenuators; purple bar: minor modulator. Human transforming growth factor alpha (hTGF, $4 \mathrm{ng} / \mathrm{ml}$ ) and a combination of p38 (SB203580, $10 \mu \mathrm{M})$ and MEK1/2 (U0126, $10 \mu \mathrm{M})$ inhibitors were used as positive and negative controls respectively. 
a.

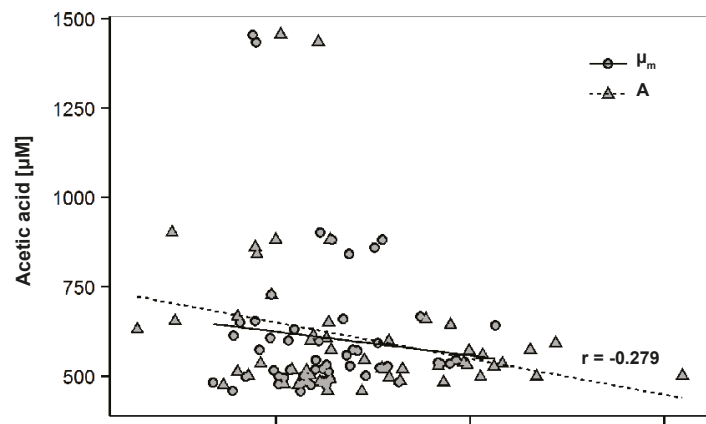

b.

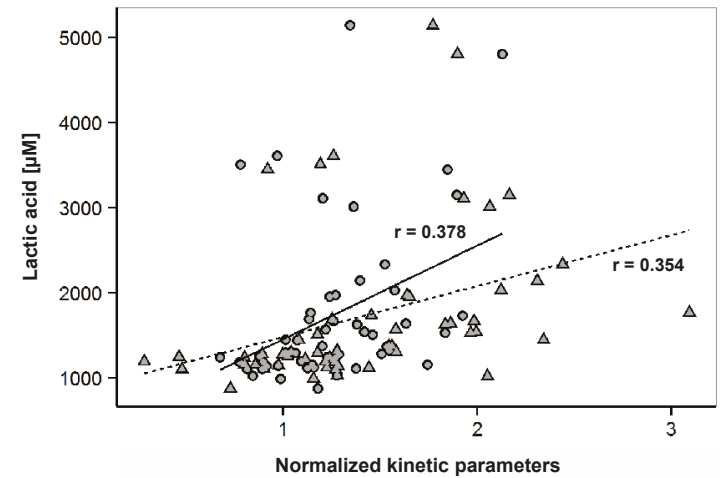

Figure S3. Correlation between metabolic end products of bacteria and re-epithelialization kinetics. Association between the concentrations of (a) acetic and (b) lactic acid with the kinetic parameters ( $A$ and $\mu_{m}$ ) of re-epithelialization assessed by Pearson correlation $(n=54)$. a.

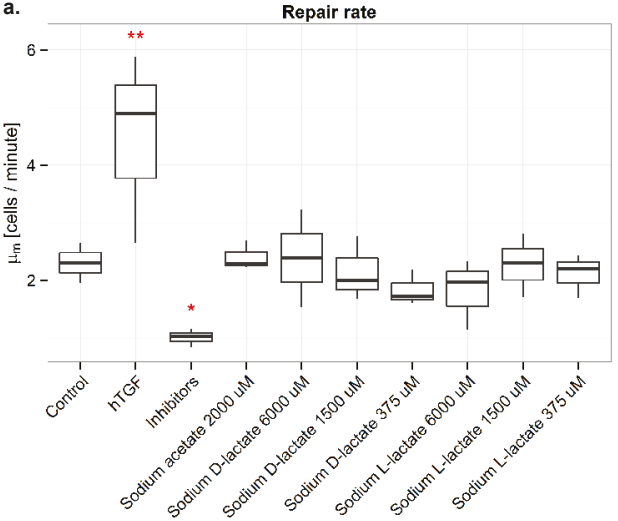

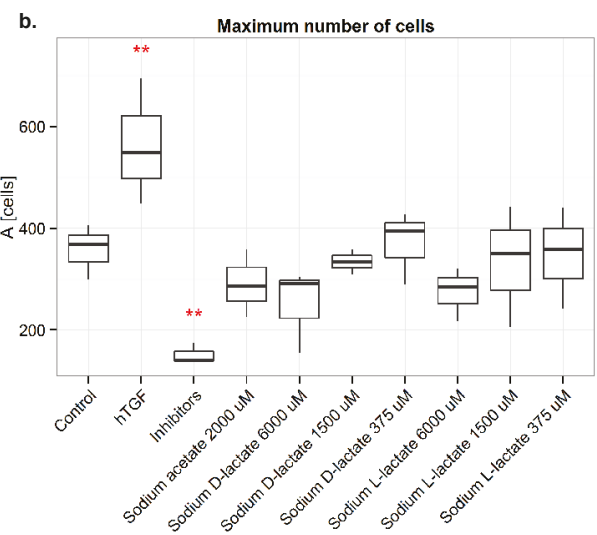

Figure S4. Re-epithelialization kinetics of cells exposed to acetate, D-lactate and L-lactate. (a) Repair rate. (b) Maximum number of cells within the scratched area after 5 hours. 
Table S1. Re-epithelialization parameter values obtained from the preliminary screening. Estimated parameters $\left(\mu_{\mathrm{m}^{\prime}} \lambda, A\right)$ and goodness of fit $\left(R^{2}\right)$ obtained after fitting the Gompertz function through the enumeration of infiltrating cells over time.

\begin{tabular}{|c|c|c|c|c|c|c|c|c|}
\hline Treatment & $\mu_{\mathrm{m}}$ & SEM_ $\mu_{m}$ & $\lambda$ & SEM_A & A & SEM_A & RMSE & $\mathbf{R}^{2}$ \\
\hline Non-treated & 2.1 & 0.2 & -1.7 & 9.2 & 373 & 16.1 & 19 & 0.97 \\
\hline Non-treated & 3.4 & 0.1 & 27.9 & 1.5 & 397 & 2.6 & 5 & 1.00 \\
\hline Non-treated & 3.3 & 0.1 & 35.8 & 2.1 & 483 & 5.2 & 7 & 1.00 \\
\hline Non-treated & 3.1 & 0.2 & -1.6 & 4.9 & 534 & 11.5 & 15 & 0.99 \\
\hline hTGFa & 6.8 & 0.4 & 2.8 & 3.4 & 758 & 9.7 & 21 & 0.99 \\
\hline hTGFa & 6.4 & 0.4 & 4.8 & 3.9 & 782 & 11.6 & 23 & 0.99 \\
\hline hTGFa & 12.8 & 1.1 & 25.1 & 2.9 & 803 & 9.7 & 26 & 0.99 \\
\hline hTGFa & 11.2 & 0.9 & 17.1 & 3.3 & 847 & 11.1 & 28 & 0.99 \\
\hline Inhibitors & 1.0 & 0.1 & 1.5 & 10.9 & 166 & 8.0 & 11 & 0.96 \\
\hline Inhibitors & 2.2 & 0.2 & 5.3 & 2.5 & 153 & 1.5 & 4 & 0.99 \\
\hline Inhibitors & 1.9 & 0.1 & 32.4 & 2.9 & 221 & 2.7 & 5 & 1.00 \\
\hline Inhibitors & 2.7 & 0.1 & 13.8 & 1.8 & 287 & 2.1 & 4 & 1.00 \\
\hline L. acidophilus NIZ0221 & 2.2 & 0.2 & -5.6 & 6.0 & 402 & 11.0 & 13 & 0.99 \\
\hline L. acidophilus NIZO221 & 4.7 & 0.3 & 5.7 & 4.3 & 710 & 12.9 & 20 & 0.99 \\
\hline L. acidophilus NIZO222 & 4.0 & 0.3 & 14.5 & 5.2 & 583 & 13.4 & 21 & 0.99 \\
\hline L. acidophilus NIZO222 & 5.5 & 0.3 & 18.3 & 2.7 & 464 & 4.9 & 12 & 1.00 \\
\hline L. acidophilus NIZO223 & 4.0 & 0.3 & 7.1 & 3.8 & 474 & 6.9 & 14 & 0.99 \\
\hline L. acidophilus NIZO223 & 3.7 & 0.2 & 7.5 & 3.6 & 552 & 8.5 & 13 & 1.00 \\
\hline L. acidophilus NIZO225 & 2.4 & 0.2 & -5.2 & 6.8 & 436 & 13.5 & 16 & 0.99 \\
\hline L. acidophilus NIZO225 & 3.9 & 0.2 & 3.9 & 4.2 & 538 & 9.0 & 15 & 0.99 \\
\hline L. acidophilus NIZO229 & 3.8 & 0.3 & -2.6 & 6.3 & 554 & 13.9 & 23 & 0.98 \\
\hline L. acidophilus NIZO229 & 2.8 & 0.2 & 1.5 & 6.1 & 450 & 11.8 & 17 & 0.99 \\
\hline L. acidophilus NIZO267 & 2.9 & 0.2 & -8.9 & 7.4 & 603 & 22.7 & 22 & 0.99 \\
\hline L. acidophilus NIZ0267 & 3.3 & 0.2 & -4.0 & 5.3 & 498 & 10.5 & 17 & 0.99 \\
\hline L. acidophilus NIZ0867 & 3.6 & 0.4 & -4.1 & 6.7 & 477 & 12.0 & 22 & 0.98 \\
\hline L. acidophilus NIZ0867 & 4.4 & 0.3 & -0.7 & 4.2 & 513 & 8.0 & 17 & 0.99 \\
\hline L. casei NIZO2928 & 2.3 & 0.1 & 20.4 & 2.6 & 288 & 3.1 & 6 & 1.00 \\
\hline L. casei NIZO2928 & 4.2 & 0.2 & 14.9 & 2.5 & 456 & 4.6 & 9 & 1.00 \\
\hline L. casei NIZO2929 & 4.5 & 0.2 & 10.2 & 2.5 & 614 & 6.4 & 11 & 1.00 \\
\hline L. casei NIZO2929 & 4.1 & 0.2 & 8.4 & 3.9 & 558 & 8.9 & 15 & 0.99 \\
\hline L. fermentum NIZ01220 & 3.5 & 0.2 & 11.4 & 3.4 & 541 & 8.4 & 12 & 1.00 \\
\hline L. fermentum NIZ01220 & 3.7 & 0.3 & -4.8 & 5.1 & 548 & 10.9 & 18 & 0.99 \\
\hline L. fermentum NIZ02517 & 4.4 & 0.3 & 8.4 & 3.4 & 463 & 6.0 & 13 & 0.99 \\
\hline L. fermentum NIZ02517 & 4.0 & 0.2 & 10.4 & 3.3 & 534 & 7.4 & 13 & 1.00 \\
\hline L. fermentum NIZ02930 & 3.0 & 0.2 & -1.1 & 6.2 & 539 & 15.4 & 19 & 0.99 \\
\hline L. fermentum NIZ02930 & 3.9 & 0.1 & 11.4 & 2.2 & 458 & 4.1 & 8 & 1.00 \\
\hline L. fermentum NIZ02931 & 2.9 & 0.2 & 15.4 & 5.4 & 440 & 11.0 & 15 & 0.99 \\
\hline L. fermentum NIZ02931 & 2.2 & 0.2 & -6.6 & 8.3 & 414 & 16.0 & 19 & 0.98 \\
\hline L. fermentum NIZ02932 & 4.7 & 0.4 & 7.6 & 4.9 & 598 & 11.5 & 21 & 0.99 \\
\hline L. fermentum NIZ02932 & 4.4 & 0.2 & 12.8 & 2.9 & 582 & 7.1 & 12 & 1.00 \\
\hline L. fermentum NIZO2933 & 3.4 & 0.2 & 11.5 & 5.4 & 535 & 13.3 & 18 & 0.99 \\
\hline L. fermentum NIZO2933 & 3.3 & 0.2 & 1.8 & 5.1 & 450 & 9.1 & 16 & 0.99 \\
\hline L. fermentum NIZO2934 & 3.6 & 0.3 & -3.0 & 6.1 & 504 & 11.8 & 21 & 0.98 \\
\hline L. fermentum NIZO2934 & 2.0 & 0.2 & -9.9 & 7.6 & 352 & 11.1 & 15 & 0.98 \\
\hline L. fermentum NIZ0307 & 1.9 & 0.2 & -4.6 & 7.8 & 299 & 9.5 & 14 & 0.98 \\
\hline L. fermentum NIZO307 & 3.4 & 0.3 & 4.5 & 4.6 & 394 & 6.8 & 14 & 0.99 \\
\hline
\end{tabular}




\begin{tabular}{|c|c|c|c|c|c|c|c|c|}
\hline Treatment & $\mu_{\mathrm{m}}$ & $S E M \_\mu_{m}$ & $\lambda$ & SEM_ $\lambda$ & A & SEM_A & RMSE & $\mathbf{R}^{2}$ \\
\hline L. paracasei NIZ02518 & 3.0 & 0.1 & 11.7 & 4.0 & 486 & 9.2 & 12 & 1.00 \\
\hline L. paracasei NIZ02518 & 2.7 & 0.3 & -11.1 & 8.9 & 436 & 15.1 & 23 & 0.97 \\
\hline L. paracasei NIZO2935 & 3.0 & 0.1 & 14.7 & 3.3 & 421 & 6.0 & 9 & 1.00 \\
\hline L. paracasei NIZO2935 & 3.0 & 0.2 & 0.4 & 6.4 & 526 & 15.8 & 19 & 0.99 \\
\hline L. paracasei NIZ02936 & 4.7 & 0.3 & 6.9 & 4.4 & 559 & 9.5 & 19 & 0.99 \\
\hline L. paracasei NIZO2936 & 5.2 & 0.3 & 3.6 & 3.2 & 605 & 7.3 & 15 & 0.99 \\
\hline L. paracasei NIZ02945 & 2.0 & 0.2 & -11.3 & 8.2 & 350 & 11.8 & 16 & 0.98 \\
\hline L. paracasei NIZO2945 & 4.3 & 0.3 & 2.0 & 4.6 & 505 & 8.7 & 18 & 0.99 \\
\hline L. plantarum NIZO2519 & 5.8 & 0.3 & 6.5 & 2.4 & 488 & 4.4 & 11 & 1.00 \\
\hline L. plantarum NIZO2519 & 3.8 & 0.3 & -0.6 & 5.7 & 603 & 14.5 & 21 & 0.99 \\
\hline L. plantarum WCFS1 & 3.9 & 0.3 & 10.4 & 5.8 & 505 & 11.9 & 21 & 0.99 \\
\hline L. plantarum WCFS1 & 3.9 & 0.3 & 3.9 & 5.1 & 530 & 10.7 & 19 & 0.99 \\
\hline L. rhamnosus GG & 5.1 & 0.3 & 2.6 & 4.2 & 644 & 10.4 & 20 & 0.99 \\
\hline L. rhamnosus GG & 3.8 & 0.2 & 13.3 & 3.7 & 518 & 8.0 & 13 & 1.00 \\
\hline L. rhamnosus NIZ02937 & 2.6 & 0.2 & 16.4 & 4.4 & 395 & 8.0 & 11 & 0.99 \\
\hline L. rhamnosus NIZO2937 & 3.2 & 0.3 & 11.0 & 5.9 & 469 & 12.0 & 19 & 0.99 \\
\hline L. salivarius NIZO2520 & 2.9 & 0.2 & 3.8 & 4.9 & 525 & 12.6 & 15 & 0.99 \\
\hline L. salivarius NIZ02520 & 4.7 & 0.3 & 3.7 & 3.8 & 482 & 6.7 & 15 & 0.99 \\
\hline L. salivarius NIZ02521 & 2.2 & 0.2 & 14.0 & 6.3 & 340 & 9.7 & 14 & 0.99 \\
\hline L. salivarius NIZ02521 & 4.8 & 0.4 & 2.0 & 4.7 & 607 & 11.0 & 21 & 0.99 \\
\hline L. salivarius NIZ02938 & 4.1 & 0.3 & 6.0 & 4.9 & 693 & 15.9 & 21 & 0.99 \\
\hline L. salivarius NIZ02938 & 3.8 & 0.3 & 8.2 & 4.7 & 537 & 10.5 & 17 & 0.99 \\
\hline L. salivarius NIZO2943 & 5.5 & 0.9 & 8.4 & 6.0 & 373 & 8.8 & 24 & 0.96 \\
\hline L. salivarius NIZ02943 & 1.5 & 0.2 & -12.1 & 12.1 & 287 & 15.6 & 18 & 0.96 \\
\hline P. gingivalis W83 & 3.9 & 0.3 & 1.7 & 3.2 & 344 & 4.1 & 10 & 0.99 \\
\hline P. gingivalis W83 & 4.0 & 0.3 & 13.5 & 2.7 & 319 & 3.4 & 9 & 0.99 \\
\hline P. gingivalis W83 & 3.9 & 0.4 & 20.0 & 3.9 & 284 & 4.4 & 11 & 0.99 \\
\hline P. gingivalis W83 & 2.2 & 0.2 & -0.6 & 3.6 & 156 & 2.1 & 6 & 0.98 \\
\hline S. bovis HSISB1 & 2.7 & 0.2 & -2.6 & 5.3 & 348 & 7.0 & 13 & 0.99 \\
\hline S. bovis HSISB1 & 2.4 & 0.2 & -4.7 & 5.4 & 406 & 9.3 & 13 & 0.99 \\
\hline S. mitis HSISM1 & 3.0 & 0.3 & -0.4 & 6.7 & 456 & 12.5 & 20 & 0.98 \\
\hline S. mitis HSISM1 & 4.9 & 0.2 & 11.0 & 2.9 & 586 & 6.8 & 13 & 1.00 \\
\hline S. mutans NIZO1215 & 2.5 & 0.3 & -1.9 & 7.8 & 331 & 9.8 & 18 & 0.97 \\
\hline S. mutans NIZO1215 & 2.7 & 0.2 & -5.2 & 4.5 & 411 & 7.3 & 12 & 0.99 \\
\hline S. salivarius HSISS1 & 2.6 & 0.2 & -0.3 & 4.8 & 383 & 7.4 & 12 & 0.99 \\
\hline S. salivarius HSISS1 & 2.5 & 0.2 & -13.0 & 8.2 & 437 & 14.5 & 20 & 0.98 \\
\hline S. salivarius HSISS2 & 2.8 & 0.2 & 5.1 & 4.4 & 430 & 8.1 & 12 & 0.99 \\
\hline S. salivarius HSISS2 & 3.6 & 0.2 & -1.7 & 4.4 & 571 & 10.7 & 16 & 0.99 \\
\hline S. salivarius HSISS3 & 3.8 & 0.3 & -8.3 & 6.6 & 590 & 15.3 & 24 & 0.98 \\
\hline S. salivarius HSISS3 & 4.0 & 0.2 & 3.2 & 4.1 & 511 & 8.2 & 15 & 0.99 \\
\hline S. salivarius HSISS4 & 3.0 & 0.2 & 1.1 & 4.5 & 527 & 11.0 & 14 & 0.99 \\
\hline S. salivarius HSISS4 & 2.7 & 0.2 & -15.7 & 9.1 & 623 & 30.8 & 25 & 0.98 \\
\hline S. salivarius MS-ileo-F1 & 4.2 & 0.1 & 13.6 & 2.7 & 686 & 8.9 & 12 & 1.00 \\
\hline S. salivarius MS-ileo-F1 & 2.8 & 0.2 & 14.7 & 5.3 & 526 & 15.7 & 16 & 0.99 \\
\hline S. salivarius MS-oral-B3 & 3.1 & 0.1 & 17.8 & 3.4 & 497 & 8.4 & 11 & 1.00 \\
\hline S. salivarius MS-oral-B3 & 3.0 & 0.2 & 4.7 & 4.5 & 430 & 8.0 & 13 & 0.99 \\
\hline S. salivarius MS-oral-D6 & 4.7 & 0.4 & 5.5 & 5.9 & 754 & 19.7 & 28 & 0.99 \\
\hline S. salivarius MS-oral-D6 & 5.0 & 0.2 & 12.4 & 3.1 & 678 & 8.7 & 15 & 1.00 \\
\hline
\end{tabular}





\title{
Chapter
}

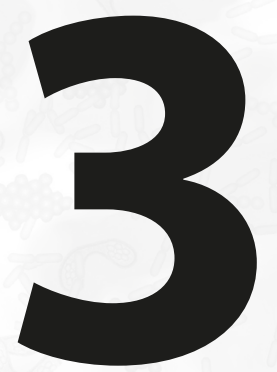

\section{KREAP: An Automated Galaxy Platform to Quantify Re-Epithelialization Kinetics}

\author{
Marcela M. Fernandez-Gutierrez ${ }^{1,2 \$}$, \\ David B.H. van Zessen ${ }^{25}$, \\ Peter van Baarlen², \\ Michiel Kleerebezem ${ }^{1,2}$, \\ Andrew P. Stubbs ${ }^{3 *}$
}

'TI Food and Nutrition, Nieuwe Kanaal 9-A, 6709 PA, Wageningen, The Netherlands.

${ }^{2}$ Host-Microbe Interactomics, Animal Sciences Group, Wageningen University \& Research, De Elst 1, 6708 WD, Wageningen,

The Netherlands.

${ }^{3}$ Department of Bioinformatics, Erasmus University Medical Centre, Wytemaweg 80, 3015 CN,

Rotterdam, The Netherlands.

\$- Both authors contributed equally 
Background: In vitro scratch assays have been widely used to study the influence of bioactive substances on the processes of cell migration and proliferation that are involved in reepithelialization. The development of high-throughput microscopy and image analysis has enabled scratch assays to become compatible with high-throughput research. However, effective processing and in-depth analyses of such high-throughput image-datasets is far from trivial and requires integration of multiple image processing and data extraction software tools.

Findings: We developed and implemented a Kinetic Re-Epithelialization Analysis Pipeline (KREAP) in Galaxy. The KREAP toolbox automatically performs image segmentation and feature extraction of each image series, followed by automatic quantification of cells inside and outside the scratched area over time. The enumeration of infiltrating cells over time is modelled to extract three biologically relevant parameters that describe re-epithelialization kinetics. The output of the tools is organized, displayed, and saved in the Galaxy environment for future consultation. Importantly, a Pearson correlation analysis confirmed a strong association between the values obtained using a multi-software approach with those obtained with the KREAP toolbox.

Conclusions: The Galaxy KREAP toolbox provides an open-source, easy-to-use, web-based platform for reproducible image processing and data analysis of high-throughput scratch assays. The KREAP toolbox could assist a broad scientific community in the discovery of compounds that are able to modulate re-epithelialization kinetics.

Keywords: Galaxy, scratch assay, high-throughput, cell migration, re-epithelialization, image analysis, workflow, modelling. 


\section{Findings}

\section{Background}

Cell migration and proliferation play an essential role in a variety of physiological processes, including embryogenesis, angiogenesis, skin and intestinal renewal, and wound repair ${ }^{155,146}$. Deregulation of these processes can contribute to the development and progression of multiple diseases such as osteoporosis, rheumatoid arthritis, vascular disease and cancer ${ }^{145}$. Therefore, studying the molecular mechanisms underlying the processes of cell migration and proliferation is not only important for obtaining fundamental scientific insight, but it is also essential for the development of effective therapeutic strategies that could modulate these processes.

The in vitro scratch assay is a well-established and widely used method to study cell migration and proliferation ${ }^{91,103,147}$. The assay is based on the introduction of a scratch into a confluent epithelial cell monolayer to create a "wounded" area. Cells migrate and proliferate into the site of injury in a process known as re-epithelialization ${ }^{69}$. This process is typically monitored by acquisition of images at the beginning and at one or more fixed time-points during re-epithelialization. The image series of a particular treatment are then compared to those of the non-treated control to determine the treatment's modulatory capacity in the healing process. The development and constant improvement of image segmentation algorithms over the past decades has enabled the transition from manual quantification of the scratch area to automated analysis that is compatible with high-throughput research ${ }^{118,148,149}$.

CellProfiler ${ }^{150}$, Image J151 and TScratch ${ }^{120}$ are freely available image analysis software tools that allow scientists with limited programming skills to conduct efficient image segmentation and feature extraction. Nevertheless, data analysis of scratch assays has been traditionally limited to the quantification of percentage of wound closure during the course of the experiment ${ }^{103}$, neglecting the kinetic information inherent of the re-epithelialization process (e.g. repair rate) and hampering the possibility of single cell migration analysis. Moreover, to optimally use the different capacities of the abovementioned software tools, scripting and parsing of data are often necessary, which require programming skills that many biologists do not have. Software such as FCS Express Image Cytometry ${ }^{152}$ and WimScratch ${ }^{153}$ provide alternatives to ease data analysis, however the former requires the purchase of a license and the latter requires payment per image analysed. Thus, there is a need in the scientific community with an interest in studying the processes of cell migration and proliferation for an open-source workflow platform that integrates different validated tools for image segmentation, visualization and data analysis.

We developed and implemented a Kinetic Re-Epithelialization Analysis Pipeline (KREAP) in Galaxy (https://galaxyproject. org//154 to deliver a web browser based application for the quantitative analysis of in vitro scratch assays. The user only need to download a virtual machine (VM) containing a fully operational KREAP Galaxy installation, upload the images from a multi-well plate experiment into the VM, and press the Execute button to automatically perform single cell segmentation and feature extraction in all images. Enumeration of cells inside and outside the scratch is also carried out automatically over the time series. The number of cells infiltrating the scratch over time is employed by KREAP to extract three biological comprehensive parameters that describe the kinetics of re-epithelialization. In addition, the user's history is saved in the VM for future consultation and the results can be easily shared with other users. Taken together, we provide a platform that enables reproducible data processing and analysis of high-throughput scratch assays: from raw images to re-epithelialization kinetics. 


\section{Implementation}

The scratch assay analysis workflow was developed within our own laboratory ${ }^{155}$ and involves a multi-software approach to acquire images, perform image analysis, visualize extracted data, and model re-epithelialization kinetics. CellProfiler 2.1.1 (http://cellprofiler.org/) was used in the original workflow and implemented in the KREAP toolbox to perform automated image segmentation and feature extraction of the image series. FCS Express 4 Plus (De Novo Software, CA, USA) was originally used to relate the features extracted by CellProfiler back to the raw images and to enumerate the cells infiltrating the scratched area over time. Since FCS Express 4 Plus requires a commercial license, we developed and implemented an $\mathrm{R}^{141}$ script in the KREAP toolbox that can automatically recognize the scratch boundaries and determine the number of cells inside and outside the scratch over time. Modelling of re-epithelialization kinetics was programmed in $\mathrm{R}$ and also implemented into the KREAP toolbox workflow. The workflow is provided in a fully operational Galaxy installation inside of a VM that can be retrieved from the Github repository and run in Microsoft Windows or Linux using the freely available VMware Workstation Player (http://www.vmware.com). Alternatively, Mac OS X users can run the VM using VirtualBox (https://www.virtualbox.org/wiki/Downloads). The source code is available as open-source via the Github repository.

\section{Analyses workflow and data handling}

For accurate image segmentation, nucleic acids must be labelled with a fluorescent marker (e.g. Hoechst 33342, SYTO ${ }^{\circledR}$ dyes, etc.) and image acquisition should be performed with a $4 X$ or $5 X$ objective to obtain a complete view of the scratched area and surrounding cells ${ }^{155}$. Images (.tif) derived from a multi-well plate must be converted into grayscale, organized in folders by well, and indexed accordingly in a separate file (.txt). An exemplary index and input files are provided in the GitHub repository (https://erasmusmc-bioinformatics.github.io/KREAP/file_formats). The folders containing the image series of each well are compressed into a .zip file, which is uploaded into the Galaxy history via the "Get data" tool together with its corresponding index file (Fig. 1). The KREAP toolbox, consisting of the "Image Analysis" and "Data-Modelling" tools, can be executed within the Galaxy platform. At the end of each processing step, the results are provided as HTML and stored in the Galaxy history for future consultation. If desired, the graphs (.png) and tables (.txt) generated by both tools can be downloaded for all wells as a .zip file by clicking the Save icon in the Galaxy history.

\section{Image Analysis tool}

Once the input files are uploaded into the Galaxy history, the "Image Analysis" tool can be executed (Fig. 1). The tool uses an image segmentation pipeline developed in the open-source software CellProfiler 2.1.1 (http://cellprofiler.org/). The individual modules contained in the pipeline carry out automated extraction of cellular features in every image. An illumination function is calculated in the first segmentation module by finding the minimum pixel intensities in blocks (recommended size: 10-20 pixels) across each image and applying a Gaussian filter ${ }^{156}$ as smoothing method. In the second module, the calculated illumination function is applied to the raw image by subtraction, resulting in better contrast between the fluorescently labelled nuclei and their background. Identification of primary objects is defined in the third module as nuclei ranging typically between 4 to 15 pixels in diameter. Identification of primary objects is performed by applying a global threshold strategy in combination with the 0tsu algorithm ${ }^{157}$ which calculates a single threshold value that classifies pixels above the threshold as foreground and below the threshold as background. Objects tend to be brighter towards the interior than towards the edges. The difference in intensity was then used to separate merged 
objects into individual ones. The last module extracts phenotypic features (e.g. size, eccentricity and mean intensity) from each object as well as their $x$ - and $y$-coordinates within the image. For optimal image segmentation results, the user can adjust the parameters for illumination correction (i.e. block size) and object identification (i.e. minimum and maximum object diameter size) per well directly in the index file. However, it is important to remark that for an objective comparison, it is recommendable to use the same parameters across wells seeded with the same cell type.

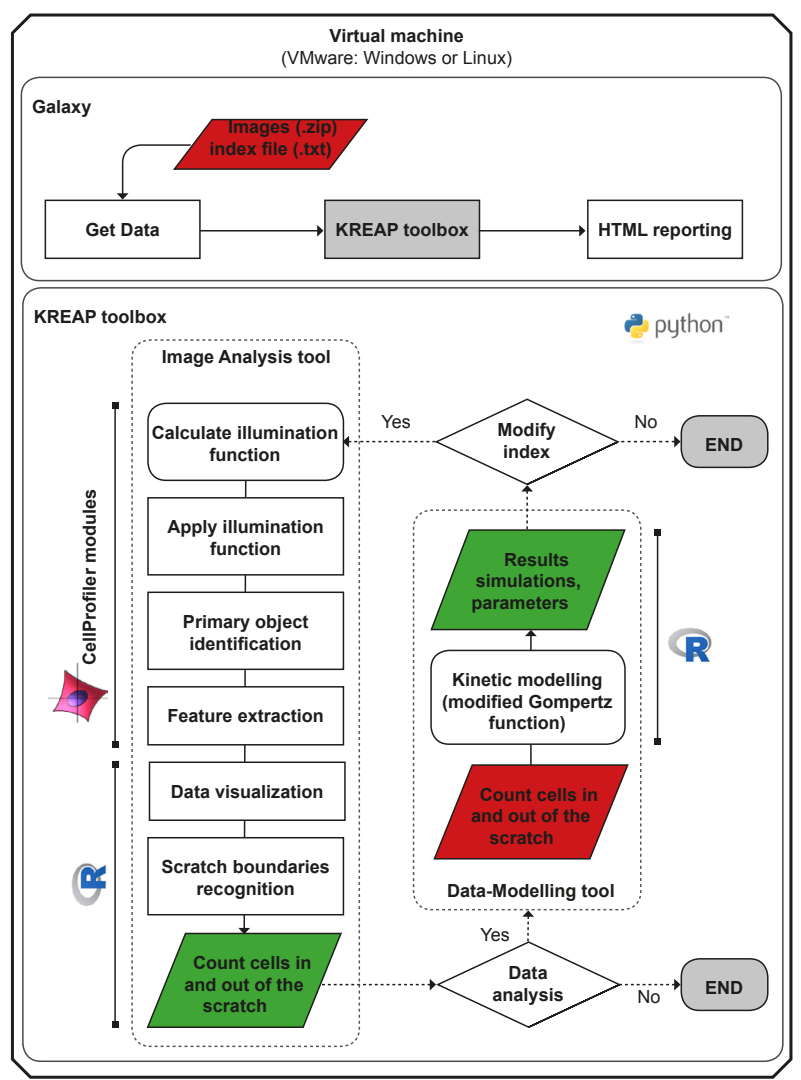

Figure 1. KREAP workflow. The virtual machine contains the KREAP toolbox and uses the graphical user interface (GUI) provided by Galaxy, including HTML reporting. The KREAP toolbox consists of the "Image Analysis" and "Data-Modelling" tools. Logos indicate the use of specialized (open source) software or programming environments in different stages of the data processing. Red parallelograms indicate input and green parallelograms indicate output. Python was used to integrate the non-Galaxy applications into Galaxy tools.

The graphical interface of Galaxy provides the user with an overview of each well within the multi-well plate (Fig. 2). The location of the identified primary objects is visualized in an interactive plot that uses a slider to move through images over time. A "compare" function is provided to visually evaluate the performance of the image segmentation pipeline by comparing its output with the raw image. Automatic identification of the scratch boundaries was programmed in $\mathrm{R}$ by finding the largest distance between cells and then expanding up and down looking for smaller gaps to avoid incorrect determination of the scratch boundaries. The total number of objects (i.e. nuclei or cells) is enumerated in each image over time and classified into objects inside or outside of the scratched area (Fig. 2). The image segmentation results are stored in the Galaxy history and can be accessed by the user in the future. Furthermore, the cellular features extracted by CellProfiler and the enumeration of objects inside or outside of the scratched area can be easily downloaded through the provided links. 


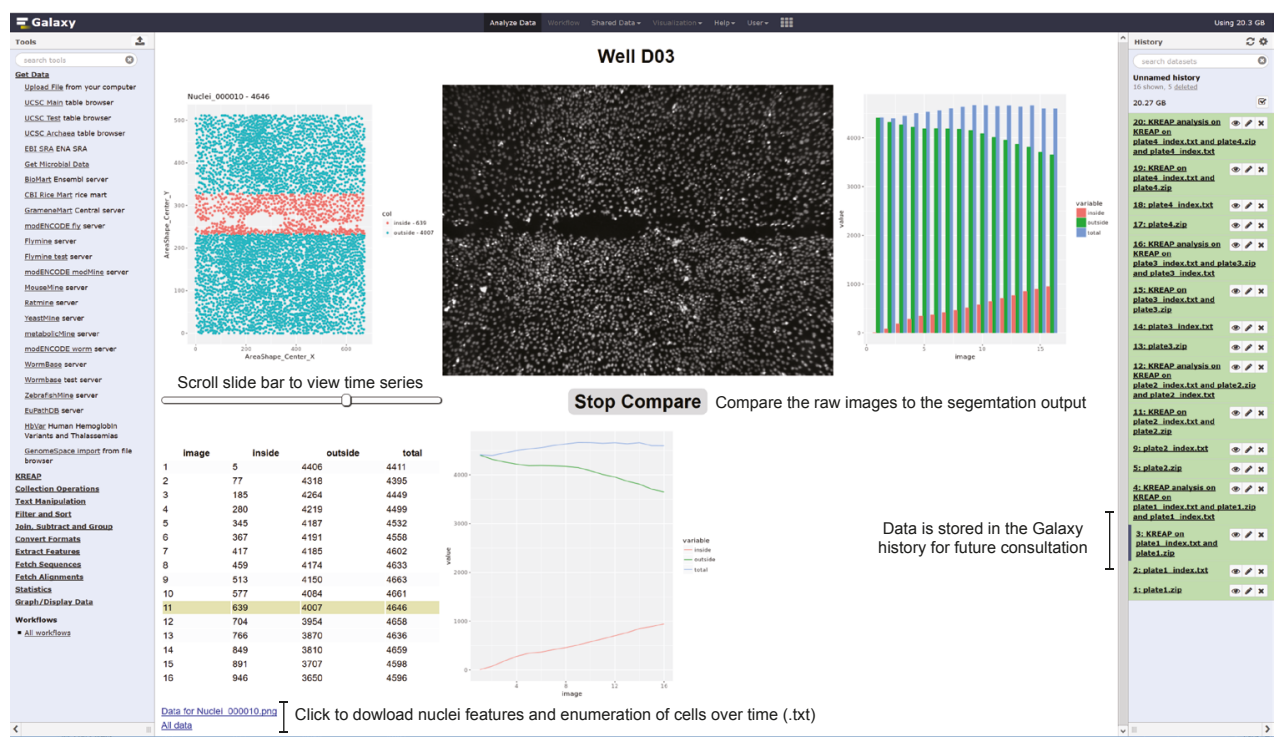

Figure 2. KREAP “Image Analysis” graphical output example. Image segmentation output can be easily compared with the raw image. Automatic recognition of scratch boundaries enables the enumeration of nuclei inside and outside of the scratched area over time.

\section{Data-Modelling tool}

The output derived from the "Image Analysis" can be used in the "Data-Modelling" tool to extract biologically relevant parameters that describe the kinetics of re-epithelialization (Fig. 1). To calculate the parameter values, the time interval between images must be entered in the index file (see https://erasmusmc-bioinformatics.github.io/KREAP/file_formats). The enumeration of infiltrating cells into the scratched area over time consistently results in a sigmoidal curve (Fig. 3), which can be successfully modelled using the modified Gompertz function ${ }^{105}$. Thus we developed and implemented an $\mathrm{R}$ script that uses a nonlinear least squares regression to fit this function through the re-epithelialization measurements ${ }^{155}$. Furthermore, the Levenberg-Marquardt algorithm ${ }^{142}$ was used in this script to reduce the sum of the squares of the errors between the modelled and measured data points in an iterative manner. In this way, we were able to obtain excellent and accurate fits, which were characterised by $R^{2}$ values close to 1 and low root-mean-square error (RMSE) values. The modified Gompertz function describes the re-epithelialization kinetics for each image series by three biologically relevant parameters: lag time ( $\lambda$ in minutes), repair rate $\left(\mu_{\mathrm{m}}\right.$ cells minute $\left.{ }^{-1}\right)$, and maximum number of cells reached within the scratched area at the plateau phase of the growth curve $(A)$. The $\lambda$ parameter represents the time in minutes required for the cells to start migrating into the scratched area. For some cell lines (e.g. (a9-22), the lag time can be very brief and the migration process may start even before image acquisition takes place according to the model outcome ${ }^{155}$. In those cases, the $\lambda$ parameter would be estimated to be zero or even have negative values and thus, the biological contribution of this parameter to the description of re-epithelialization kinetics would be limited, but its calculation essential for obtaining a good fit of the model. Apart from the lag time or $\lambda$ parameter, the $\mu_{\mathrm{m}}$ and the A parameters can be used to compare the re-epithelialization kinetics of cells treated with a specific substance to that of the non-treated control. The combinatorial approach of incorporating the $\mu_{\mathrm{m}}$ and the A parameters into a single performance value $\left(\mu_{\mathrm{m}}{ }^{*} \mathrm{~A}\right)$ can be 
useful to identify potential stimulators and attenuators of re-epithelialization. For this calculation, the $\mu_{\mathrm{m}}$ and the $A$ values of each treatment are normalized against the corresponding average values of the non-treated control ${ }^{155}$.

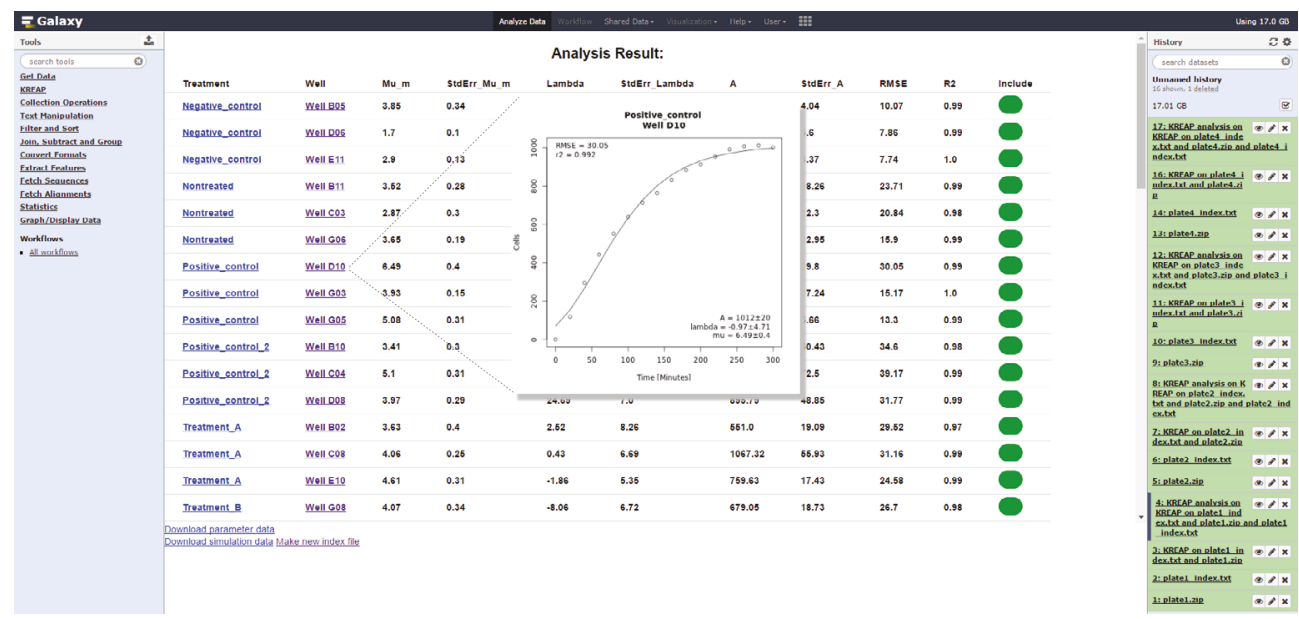

Figure 3. KREAP "Data-Modelling" tool output example. Re-epithelialization kinetics described by the $\lambda$, $\mu_{\mathrm{m}}$ and A parameters. The parameter values, simulation data and re-epithelialization curves per replicate are provided in an HTML report and are also available for download through the available links.

\section{Validation with published sub-data set}

To validate the performance of KREAP in comparison with the original workflow that uses a multi-software approach, we re-analysed a sub-data set of one of our previous studies using the KREAP toolbox in the Galaxy platform. Data processing was performed on a Windows desktop with an $\operatorname{Inte} \mathrm{I}^{\circledR} \mathrm{Core}^{\mathrm{TM}} \mathrm{i} 7$-3970X processor with 4 cores at $3.50 \mathrm{GHz}$ and $4 \mathrm{~GB}$ of RAM. Both KREAP and the original workflow make use of CellProfiler for automated image segmentation and feature extraction. Data visualization and enumeration of infiltrating cells over time was determined previously using the commercially available FCS Express 4 Plus (De Novo Software, CA, USA) software tool, which we now replaced by a freely available $\mathrm{R}$ script ${ }^{155}$. The location of the identified objects at the beginning of the assay was plotted in a scatterplot after which a gate was manually placed on the scratched area and a batch process was setup to record the number of infiltrating cells over time for each well ${ }^{155}$. Processing of a multi-well plate experiment consisting of 60 wells and 16 time points (960 images in total) would typically take around 3 to 4 hours for an experienced user to complete with this protocol. In addition, programming skills are required to extract the parameter values that described re-epithelialization kinetics. In contrast, the KREAP toolbox can automatically perform the complete analysis from raw images to quantification of reepithelialization kinetics in less than 30 minutes. Furthermore, a Pearson correlation analysis confirmed the association between the values obtained in the previous study with those obtained with the KREAP toolbox for both the $\mu_{\mathrm{m}}$ and $\mathrm{A}$ parameters with $R^{2}$ values of 0.87 and 0.83 respectively (Fig. 4). 

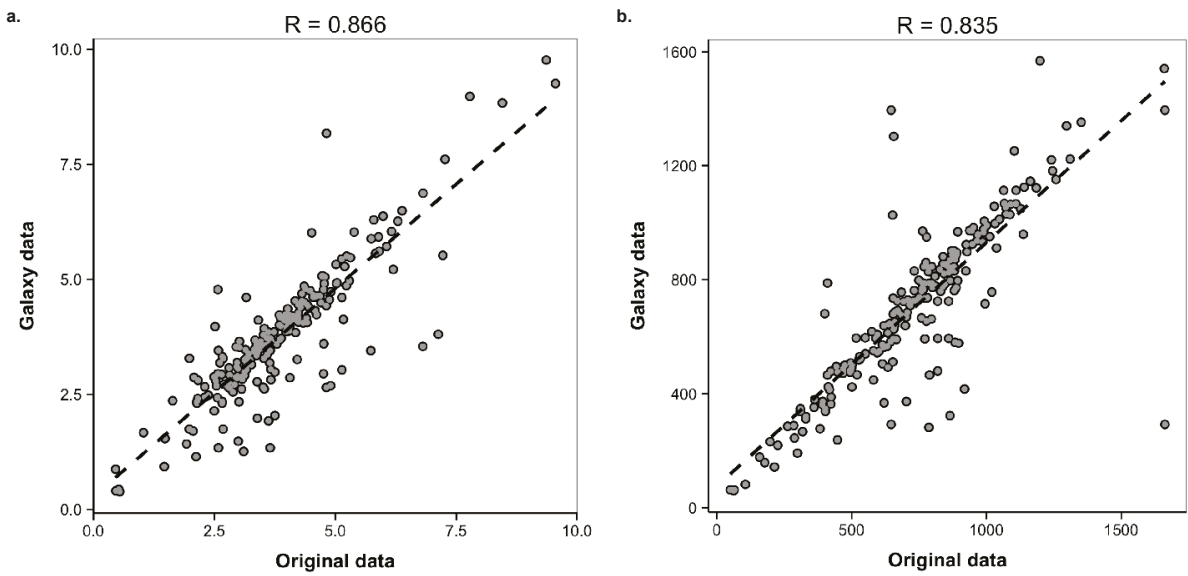

Figure 4. Association between the parameter values originated with multi-software approach and the KREAP toolbox. (a) Repair rate ( $\mu_{m}$ parameter, cells minute ${ }^{-1}$ ) and (b) Maximum number of cells (A parameter, cells). The association of the parameter values was evaluated by Pearson correlation analysis $(n=215)$ and for both cases a positive and significant correlation was found $(P<0.0001)$.

\section{Identification of detrimental effects on re-epithelialization and troubleshooting}

The modified Gompertz function was developed to model growth (i.e. positive sigmoidal curves), but identification of detrimental effects on re-epithelialization kinetics is still possible through inspection of the curves generated with the measured and modelled data points. In the first example (Fig. 5a), cells had migrated into the scratched area until the plateau was reached, but this was followed by a reduction in the number of infiltrating cells over time as a result of cell death leading to a low $R^{2}$ value. The KREAP "Data-Modelling" tool flags $R^{2}$ values lower than 0.9 to be inspected by the user. In the second example (Fig. $\mathbf{5 b}$ ), cell migration was strongly inhibited by the treatment and therefore, only very few cells migrated into the scratched area. These curves cannot be modelled by the modified Gompertz function and therefore, the parameter values describing re-epithelialization kinetics will not be retrieved. The KREAP "Data-Modelling" tool flags the wells in which the parameter values could not be retrieved and the user can decide to exclude the treatment from the analysis by clicking the icon in the Include column and have a new index file automatically created through the provided link (Fig. 5c). Finally, if the assay is too short for a particular treatment to reach the plateau phase of the growth curve (Fig. 5d), the estimation of the parameter values would be overestimated, resulting in outliers. In this case, the user is advised to extend the timeframe of the assay. 
a.

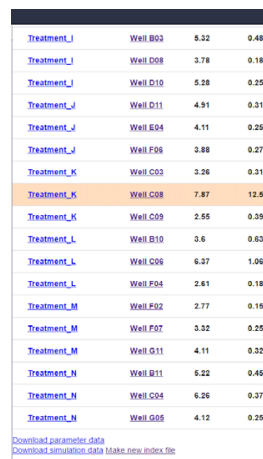

c.

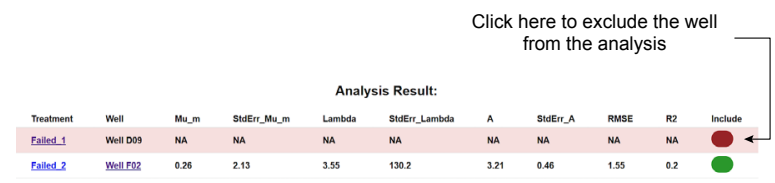

b.

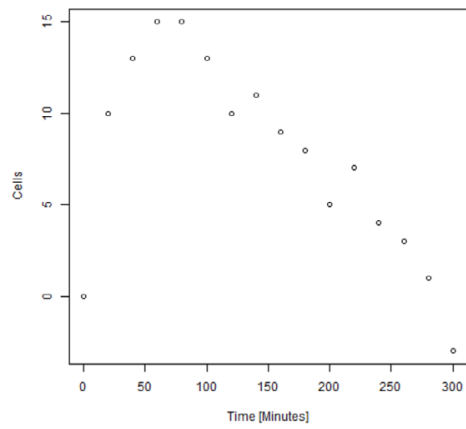

d.

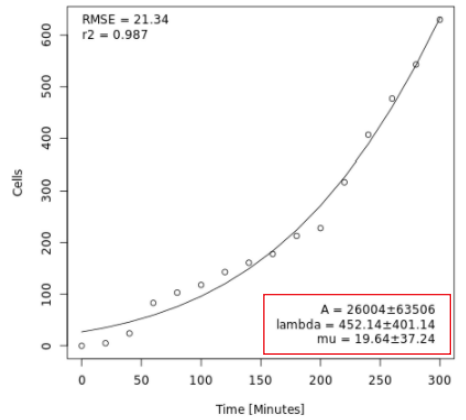

Figure 5. Identification of detrimental effects and outliers using KREAP. (a) Detrimental effect on re-epithelialization characterized by a low $R^{2}$ value as a result of extensive cell death after reaching the plateau of the growth curve. (b) Strong inhibitory effect results in migration of very few cells into the scratched area and as a result the parameter values that describe re-epithelialization cannot be retrieved. (c) The user can decide to exclude certain wells of the analysis by pressing the icon in the Include column. (d) Overestimation of the parameter values can result from growth curves that do not reached the plateau phase in the timeframe of the assay.

\section{Conclusions}

A key aspect of high-throughput microscopy research is to convert the raw images into quantitative and biologically comprehensive data. This step often requires multiple software tools, programming skills or purchase of costly software to aid in the image processing and data analysis. The KREAP toolbox integrates multiple validated tools for image segmentation, visualization and data analysis of high-throughput scratch assays in Galaxy. Our KREAP toolbox in Galaxy provides an open-source web-based platform that enables scientists that lack sophisticated programming skills to perform the complete analysis starting with the raw images and ending with the quantified kinetics. Furthermore, the graphical user interface of Galaxy provides an easy-to-use environment that organizes, displays, and saves the results of every experiment as part of the user history. This is the first open-source application to provide an "end to end" integrated analytical high-throughput screening platform that is useful for scientists who are interested in the discovery and mechanistic analysis of compounds that can modulate re-epithelialization kinetics. 


\section{Availability and Requirements}

$0 \quad$ Project name: KREAP (Kinetic Re-Epithelialization Analysis Pipeline)

$0 \quad$ Project home page: https://erasmusmc-bioinformatics.github.io/KREAP/

$0 \quad$ Operating system: Unix-based Operating Systems

$0 \quad$ Programming languages: Python, R programming language

$0 \quad$ License: Free

$0 \quad$ Any restriction to use as non-academic: none

$0 \quad$ Virtual machine accessibility: via the GitHub repository

\section{Availability of Supporting Data}

The source code and supporting data for the presented analyses are publicly available in the project home page. 


\title{
Chapter
}

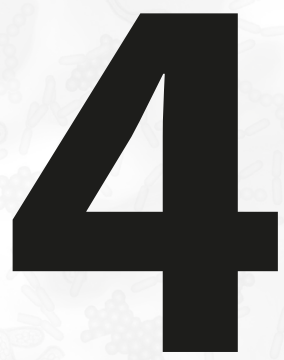

\section{High-Throughput Screening Model to Quantify Re-Epithelialization Kinetics}

\author{
Marcela M. Fernandez-Gutierrez ${ }^{1,2}$, \\ Peter P.J. Roosjen ${ }^{3}$, \\ David B.H. van Zessen ${ }^{4}$, \\ Jerry M. Wells ${ }^{2}$, \\ Peter van Baarlen², \\ Andrew P. Stubbs ${ }^{4}$, \\ Michiel Kleerebezem ${ }^{1,2^{*}}$
}

\footnotetext{
${ }^{1}$ TI Food and Nutrition, Nieuwe Kanaal 9-A, 6709 PA, Wageningen, The Netherlands.

${ }^{2}$ Host-Microbe Interactomics Group, Department of Animal Sciences, Wageningen University \& Research,

De Elst 1, 6708 WD, Wageningen, The Netherlands.

${ }^{3}$ Laboratory of Geo-Information Science and Remote Sensing, Wageningen University \& Research, Droevendaalsesteeg 3, 6708 PB, Wageningen, The Netherlands.

${ }^{4}$ Department of Bioinformatics, Erasmus University Medical Centre, Wytemaweg 80, 3015 CN,

Rotterdam, The Netherlands.
} 
In vitro scratch assays provide an attractive method to study the regulation and mechanisms of epithelial cell proliferation and migration that are involved in wound repair. The assay is performed by introducing a scratch into a confluent cell monolayer and monitoring the re-epithelialization of the "wound" by acquiring images over time. Despite improvements to the method over the past years, high-throughput screenings often require the integration of multiple image analysis and data extraction software tools. We developed a high-throughput image-based scratch assay and an automated processing pipeline that employs a mathematical model to describe re-epithelialization kinetics. We implemented a processing pipeline in Galaxy called KREAP that provides an open source web-based platform to enable scientists from diverse backgrounds to perform reproducible and quantitative repair data analyses in less than 45 minutes. 


\section{Introduction}

In vitro scratch assays have been frequently employed to test the influence of bioactive substances on the processes of cell migration and proliferation $75,79,80,119,147,15,159$. These assays are carried out in multi-well plates and commonly rely on the manual introduction of a scratch in a confluent epithelial cell monolayer using a sterile pipette tip, razor blade, scraper or needle. The remaining epithelial cells will then migrate and proliferate into the scratched area until cell-cell contacts are re-established ${ }^{103,160}$. In order to standardize and increase the throughput, significant improvements have been made to the in vitro wounding tools and methods, including the use of hand-guided pin or pipette tip arrays, robotically driven scratching-pin devices, chemically induced wounds, and laser ablation ${ }^{118,149}$. Furthermore, the assays have evolved from examining images acquired manually at fixed-time points to the use of automated fluorescent microscopy to monitor live cells expressing fluorescent markers ${ }^{103}$. Likewise, manual quantification of the scratched area has been substituted by the implementation of automated image segmentation algorithms that facilitate the analysis of high-throughput screenings ${ }^{120}$. Despite all these improvements, data analysis has been usually limited to the calculation of wound closure over time ${ }^{120,148}$, disregarding the kinetic information inherent to the healing process. In addition, effective processing and quantification of high-throughput image-based assays involves the integration of multiple image analysis and data extraction software tools that often require computer programming skills and/or a commercial license, increasing total experimental costs ${ }^{155}$. Here we present the procedure of an optimized high-throughput scratch assay using high-content microscopy and a mathematical model to quantify the kinetics of epithelial wound repair in individual wells through the calculation of biologically relevant parameters. The assay can be used for screening chemical compounds, bacterial suspensions ${ }^{155}$, and other bioactive substances for their effect on re-epithelialization kinetics. Furthermore, we developed and implemented a Kinetic Re-Epithelialization Analysis Pipeline (KREAP) that we implemented in Galaxy, thus providing an end-to-end workflow integrating different validated tools for reproducible image analysis, cell enumeration, reepithelialization modelling, and HTML reporting ${ }^{161}$. KREAP is available for download and use as a virtual machine for both large and small-scale laboratories (https://erasmusmc-bioinformatics.github.io/KREAP/).

\section{Development of the protocol: Quantification of re-epithelialization kinetics}

In order to quantify the re-epithelialization kinetics of gingival cells (i.e. Ca9-22 cell line) exposed to different bacterial suspensions using our standardized scratch assay, we developed an automated image segmentation pipeline using CellProfiler 2.1.1 (http://www.cellprofiler.org/) which identifies and records the location of each cell within the well over time ${ }^{155}$. Given that the number of cells moving into the scratched area over time can be represented by a sigmoidal curve, we used a modified form of the Gompertz function ${ }^{105}$ to quantify the kinetics of wound repair. The modified Gompertz function consists of three biologically comprehensible parameters: lag time ( $\lambda$ parameter, in minutes), repair rate $\left(\mu_{m}\right.$ parameter, cells/minute), and maximum number of infiltrating cells at the plateau of the growth curve (A parameter, in cells). This function was fitted through the number of infiltrating cells over time by a nonlinear least squares regression using the Levenberg-Marquardt algorithm ${ }^{142}$ to reduce the sum of the squares of the errors between the modelled and measured data points in an iterative manner. Using this approach, we were able to obtain excellent and accurate fits, characterized by a goodness of fit $\left(R^{2}\right)$ with values close to one and low root-mean-square errors (RMSE), respectively (Fig. 1). 


\section{Experimental design}

Selection of suitable cell line. For automated microscopy, it is preferable to select a cell line that grows in a single layer of cells, rather than in multilayers. Furthermore, it is important to evaluate the expression of relevant receptors (e.g. Tolllike receptors, epidermal growth factor receptor, etc.) in the candidate cell line as well as its responsiveness to chemical stimuli. Cells with a long doubling time (e.g. (aco-2 cell line) are less suitable for this set-up as they will require long periods of imaging. In addition, epithelial cell lines that form strong tight-junctions (e.g. (aco-2 cell line) may not allow the introduction of regular and reproducible scratches and for that reason are not ideal for this assay.

Controls. Titration curves of the controls need to be carried out on each cell line to determine the optimal concentrations resulting in a large dynamic range between the positive and negative controls (Fig. S1 and Box 1). A non-treated control should be included as a reference to determine whether the screened substances have a stimulating or attenuating effect on re-epithelialization kinetics.

$Z^{\prime}$ factor calculation. The overall quality of each assay can be assessed by calculation of the $Z^{\prime}$ factor ${ }^{106}$ defined by the means and variability of the positive and negative controls (Box 1).

\section{Limitations}

The modified Gompertz function was implemented to describe positive growth curves. Therefore, if the growth curves resulting from a particular treatment does notresemble a sigmoidal curve, the modified Gompertz function cannotbe used to calculatethekineticparameters. Thisis oftenthecase oftreatmentsthatleadtostronginhibitoryeffectsonre-epithelialization where only few cells migrate into the scratched area during the timeframe of the assay. The KREAP "Data-Modelling" tool flags the wells from which the parameter values were not retrieved and the user can then examine the curves resulting from the measured data points over time as well as the image segmentation output (also see https://erasmusmcbioinformatics. github.io/KREAP/use_kreap_model_error). Some treatments may induce cell death after the plateau of the growth curve has been reached, leading to a reduction in the number of infiltrating cells towards the end of the assay and lower $R^{2}$ values $(<0.9)$. These detrimental effects can be identified by inspection of the re-epithelialization curves generated by the KREAP tool that compares the measured and modelled data points. $R^{2}$ values lower than 0.9 are also flagged by KREAP to alert the user to examine the re-epithelialization curves. 


\section{Box 1. Assay Quality Assessment}

Positive controls included human epidermal growth factor (hEGF) and transforming growth factor a (hTGFa), which both bind to the epidermal growth factor receptor (EGFR) and act as mitogenic and cell motility factors ${ }^{162}$. Specific inhibitors of p38 mitogen-activated protein kinase (SB203580) and MEK1/2 (U0126) can be used both separately or in combination to suppress wound repair by blocking the activation of cell migration ${ }^{138}$ and proliferation ${ }^{149,163}$ pathways, respectively (Fig. S2).

Calculation of the $Z^{\prime}$ factor can be used to assess the overall quality of each independent run. The $Z^{\prime}$ factor ${ }^{106}$ is based on the ratio between the variability of the controls (i.e. standard deviation, $\sigma$ ) and the absolute difference between the means $(\mu)$ of the positive and negative controls. The difference between the controls defines the dynamic range of the assay signal. The $Z$ ' factor can be calculated using the following equation:

$$
Z^{\prime}=1-\frac{\left(3 \sigma_{c+}+3 \sigma_{c-}\right)}{\left|\mu_{c+}-\mu_{c-}\right|}
$$

If the $Z^{\prime}$ factor values are negative it generally indicates that the assay conditions are not optimal and cannot be used to generate useful data. Values between 0 and 0.5 indicate that the separation band between the controls is small, whereas values between 0.5 and 1 indicate a large dynamic range and a likely successful assay.

\section{Materials}

\section{Reagents}

- Oral epithelial cell lines, e.g. Ca9-22 (National Institute of Biomedical Innovation JCRB Cell Bank, cat. no. JCRB0625), H0-1-N-1 (National Institute of Biomedical Innovation JCRB Cell Bank, cat. no. JCRB0831)

- Skin keratinocytes, e.g. HaCat (AddexBio, cat. no. T0020001)

- !CAUTION. The cell lines should be routinely checked to ensure that they are authentic and not cross-contaminated (e.g. using short-tandem repeat analysis) or infected with mycoplasma (PCR-based detection).

- Cell culture medium, e.g. Dulbecco's Modified Eagle Medium (DMEM), DMEM/F12 (1:1) or Keratinocyte-Serum Free Medium (SFM) Gibco, cat. no. 61965-026, 31331-028, 17005-034)

- Heat inactivated fetal calf serum (FCS) (Gibco, cat. no. 10500)

- Penicillin-Streptomycin 100X (Sigma, P0781-20ML)

- $0.25 \%$ Trypsin EDTA (1x) (Gibco, 25200-056)

- Sterile phosphate-buffered saline (PBS) (Gibco, 18912-014)

- CellTracker ${ }^{\text {TM }}$ Red CMTPX (Molecular Probes, cat no. (34552) (optional)

- Hoechst 33342 (Molecular Probes, cat no. H1399)

- ! CAUTION. Hoechst stains are known mutagens and should be handled with care.

- Human transforming growth factor a (hTGFa; R\&D Systems, cat. no. 239-A-100)

- Human epidermal growth factor (hEGF, Gibco, cat. no. PHG0311)

- Inhibitor of p38 (SB203580; Cell Signaling Technology, cat. no. 5633)

- Inhibitor of MEK1/2 (U0126, Cell Signaling Technology, cat. no. 9903)

- ! CAUTION. The MEK1/2 inhibitor (U0126) may cause irritation to eyes, skin, and mucous membranes. 


\section{Equipment}

- $75 \mathrm{~cm}^{2}$ cell culture flasks (Corning Incorporated, cat. no. 430641U)

- Tissue culture-treated 96-well plates (BD Falcon, cat. no. 353219)

- Tissue culture-treated 96-well plates with flat bottom (Corning Incorporated, cat. no. 3599)

- Tissue culture-treated 96-well plates with round bottom (Corning Incorporated, cat. no. 3799)

- Multichannel pipette, 8-channel, $100 \mu l$ (Eppendorf Research Plus, cat. no. 3122000035)

- Disposable serological pipette $5 \mathrm{ml}, 10 \mathrm{ml}$ and $25 \mathrm{ml}$ (Corning Incorporated, cat. no. 4051, 4101, and 4251)

- Reagent Reservoir $50 \mathrm{ml}$ (Corning Incorporated, cat. no. 4870)

- Pipette $10 \mu \mathrm{l}, 200 \mu \mathrm{l}$ and $1 \mathrm{ml}$ (Eppendorf Research Plus, cat. no. 3120000020, 3120000054, and 3120000062)

- Filter sterile tips $10 \mu \mathrm{l}, 200 \mu \mathrm{l}$ and $1 \mathrm{ml}$ (Corning Incorporated, 4807, 4823, and 4809)

- Tips for multichannel pipette 2-200 $\mu$ l (Eppendorf, cat. no. 0030073.428)

- Counting chamber (Brand, cat. no. 719520)

- HTSScratcher (Peira, https://www.peira.be/platforms/oncology-platforms)

- Laminar flow cabinet

- Laboratory vacuum pump

- Inverted microscope, e.g. Zeiss, Axiovert 40CFL

- Automated fluorescent microscope with controlled temperature and atmosphere, e.g. BD Pathway 855 (BD Biosciences), IN Cell Analyzer 2500HS System (GE Healthcare Life Sciences).

- Humidified tissue culture incubator (Thermo-Scientific, Forma Direct Heat $\mathrm{CO}_{2}$ Incubator)

- KREAP (Kinetic Re-epithelialization Analysis Pipeline) virtual machine (https://erasmusmc-bioinformatics.github. io/KREAP/)

\section{Reagents setup}

Cell growth media. Add penicillin-streptomycin to the cell culture media to a final concentration of $1 x$ and the inactivated FCS to a final concentration of $10 \%$. Store at $4^{\circ} \mathrm{C}$.

Hoechst 33342 stock solution ( $10 \mathrm{mg} / \mathrm{ml})$ and working solution $(1 \mathrm{mg} / \mathrm{ml})$. To prepare the stock solution, dissolve 100 $\mathrm{mg}$ lyophilized dye in $10 \mathrm{ml}$ dimethyl sulfoxide (DMSO). Divide the stock solution into aliquots to avoid repeated freeze-thaw cycles. Store at $-20^{\circ} \mathrm{C}$ protected from light. This solution is stable for at least one year according to the manufacturer. To prepare the working solution, add $100 \mu \mathrm{l}$ of the stock solution into $900 \mu \mathrm{l} \mathrm{PBS}$ and store at 2- $6^{\circ} \mathrm{C}$ for a maximum of three months.

CellTracker $^{\mathrm{Tm}}$ Red CMTPX stock solution (100 $\left.\mu \mathrm{M}\right)$. Dissolve $50 \mu \mathrm{g}$ lyophilized dye in $730 \mu$ DMSO. Divide the solution into aliquots to avoid repeated freeze-thaw cycles. Store at $-20^{\circ} \mathrm{C}$ protected from light. This solution is stable for at least three months. Lyophilized dye is stable for at least one year according to the manufacturer.

hTGFa stock solution (10 $\mu \mathrm{g} / \mathrm{ml}$ ). Reconstitute $100 \mu \mathrm{g} \mathrm{hTGFa} \mathrm{in} 1 \mathrm{ml} 10 \mathrm{mM}$ filter-sterilized acetic acid. Add this solution into $9 \mathrm{ml}$ DMEM to obtain the stock solution. Divide into aliquots and store at $-20^{\circ} \mathrm{C}$ to avoid repeated freeze-thaw cycles. According to the manufacturer, this solution is stable for at least three months.

hEGF stock solution (10 $\mu \mathrm{g} / \mathrm{ml})$. Reconstitute $100 \mu \mathrm{g} \mathrm{hEGF} \mathrm{in} 1 \mathrm{ml}$ sterile PBS. Add this solution into $9 \mathrm{ml}$ DMEM containing heat inactivated FCS to obtain the stock solution. Divide into aliquots and store at $-20^{\circ} \mathrm{C}$ to avoid repeated freeze-thaw cycles. According to the manufacturer, this solution is stable for one year. 
SB203580 stock solution (10 mM). Reconstitute $5 \mathrm{mg}$ of lyophilized inhibitor in $1.32 \mathrm{ml} \mathrm{DMSO}$. Divide into aliquots and store at $-20^{\circ} \mathrm{C}$ to avoid repeated freeze-thaw cycles.

U0126 stock solution (10 mM). Reconstitute $5 \mathrm{mg}$ of lyophilized inhibitor in $1.32 \mathrm{ml}$ DMSO. Divide into aliquots and store at $-20^{\circ} \mathrm{C}$ to avoid repeated freeze-thaw cycles.

\section{Equipment setup}

Fluorescence Microscope. Hoechst 33342 and CellTracker ${ }^{\mathrm{TM}}$ Red CMTPX images should be acquired using an excitation filter of $350 \mathrm{~nm}$ and $577 \mathrm{~nm}$, respectively. Bright-field images serve as visual control for cell morphology and vitality. The microscope should be programmed to acquire fluorescent and bright-field images of the same field of each well every 20 minutes (recommended, but can be adjusted according to the user's needs) using a $4 x$ objective (40x magnification).

\section{Procedures}

\section{Cell culturing and seeding of cells. TIMING 3-7 days depending on the cell line}

1. Grow low-passage epithelial cells to $70-80 \%$ confluence in $75 \mathrm{~cm}^{2}$ tissue culture flasks. Ca9-22 and HaCat cells should be grown in DMEM supplemented with $10 \% \mathrm{FCS}, 100 \mathrm{U} / \mathrm{ml}$ penicillin and $100 \mu \mathrm{g} / \mathrm{ml}$ streptomycin. HO1-N-1 cells should be cultured in DMEM/F12 (1:1) supplemented with 10\% FCS, $100 \mathrm{U} / \mathrm{ml}$ penicillin and $100 \mu \mathrm{g} / \mathrm{ml}$ streptomycin. CRITICAL STEP. Always wear gloves when handling cells and work in a laminar flow cabinet. The cell culture environment should be kept clean, i.e. in absence of bacteria, molds and mycoplasma.

2. Discard the growth culture media and wash the cells with $6 \mathrm{ml}$ PBS.

3. Discard the PBS and add $2 \mathrm{ml}$ trypsin/EDTA and incubate at $37^{\circ} \mathrm{C}$ for $3-5$ minutes. CRITICAL STEP. Verify complete detachment of the cells under an inverted microscope.

4. Add $8 \mathrm{ml}$ of supplemented growth media into the flask and gently resuspend the cells by gently pipetting up and down.

5. Take an aliquot of the cell suspension and determine the cell counts using a counting chamber. Dilute the cell suspensions in the corresponding growth media. The Ca9-22 cell suspension should be diluted to obtain $3.5 \times 10^{5}$ cells/ml; whereas H0-1-N-1 and HaCat cell suspensions should be diluted to obtain $3.0 \times 10^{5}$ cells $/ \mathrm{ml}$.

CRITICAL STEP. The number of cells required to form an intact monolayer within the standardized timeframe depends on the cell type and thus, should be pre-determined for each cell line.

6. Mix the cell suspension gently and add the suspension into a reagent reservoir.

7. Using a multichannel pipette, add $100 \mu \mathrm{l}$ of the cell suspension into each well of a 96 -well plate with flat bottom (BD Falcon; recommended for imaging using the BD Pathway 855 Bioimaging System).

8. Incubate for 16 hours in a humidified incubator at $37^{\circ} \mathrm{C}$ with $5 \% \mathrm{CO}_{2}$.

\section{Cell starvation. TIMING 2 hours (might vary for other cell lines)}

9. Replace the growth media by $100 \mu$ starvation media (i.e. FCS-free media) by aspiration of the culture medium without disturbing the cell monolayers. HaCat cells should be starved in keratinocyte serum-free medium (SFM). Starvation of Ca9-22 cells should be performed in FCS-free DMEM/F12, whereas H0-1-N-1 cells should be starved 
in FCS-free DMEM. SFM is not recommended when using Ca9-22 or H0-1-N-1 cells as it was shown to promote significant cell migration and proliferation in our setup that restricted the detection of exogenously added stimulatory compounds.

CRITICAL STEP. The duration and the media used during starvation needs to be optimized for each cell line. FCS deprivation should minimize cell migration and proliferation without inducing apoptosis or cell detachment.

\section{? TROUBLESHOOTING}

10. Incubate the cells (Ca9-22, $\mathrm{H} 0-1-\mathrm{N}-1$ or $\mathrm{HaCat}$ cells) for 2 hours in a humidified incubator at $37^{\circ} \mathrm{C}$ with $5 \% \mathrm{CO}_{2}$.

\section{Preparation of master plate with treatment and control samples. TIMING 1.5 hours}

11. Prepare the treatments that you wish to test for their effect on re-epithelialization kinetics. An example of bacterial preparations is provided in Box 2. Initial screenings can be performed in duplicates, but refined studies should be carried out with at least three technical replicates in two or more independent experiments.

CRITICAL STEP. All treatments should be diluted in the starvation media.

12. Prepare the positive and negative controls as indicated in Table $\mathbf{1 .}$

CRITICAL STEP. Optimal concentrations of the controls should be determined by performing a titration for the target cell lines (Fig. S1) and should result in a large dynamic range between the positive and negative controls (Box 1).

\section{? TROUBLESHOOTING}

13. Prepare a master plate by adding $120 \mu \mathrm{l}$ of each sample into the corresponding well.

CRITICAL STEP. Exclude outer wells to avoid any possible edge-specific artifacts, e.g. due to evaporation of the medium. It is also recommended to add the samples into the wells in a randomized manner.

\section{Box 2. Preparation of bacterial suspensions}

High-throughput screening of viable bacteria ${ }^{155}$ often require preparation of $15 \%$ glycerol stocks at a specific growth stage prior to the experiment.

\section{Preparation of $15 \%$ (v/v) glycerol stocks}

1. Culture the bacterial strains until the desired growth stage (e.g. early stationary stage) under the required atmospheric conditions.

2. Take $700 \mu$ l of the bacterial suspension into a cryovial (Corning Incorporated, cat. no. 430488) and add $300 \mu$ l glycerol $50 \%$ (final concentration 15\%).

3. Store at $-80^{\circ} \mathrm{C}$ until used.

\section{Preparation of bacterial suspensions for screening}

1. Thaw the bacterial suspensions completely by placing them at room temperature.

2. Pellet the bacteria by centrifugation ( $4,000 \times \mathrm{g}, 7$ minutes at room temperature).

3. Remove the spent media and resuspend the bacteria in $700 \mu$ starvation media.

4. Dilute the bacterial suspension in starvation media until the required multiplicity of infection (MOI) is obtained in 100 $\mu \mathrm{l}$ of the bacterial suspension. For example, to obtain an $\mathrm{MOI}$ of 10 with an epithelial density of $3.5 \times 10^{4}$ cells per well, prepare a bacterial suspension containing $3.5 \times 10^{6}$ bacterial cells per ml and add $100 \mu \mathrm{l}$ to each well. 
Table 1. Working concentrations of positive and negative controls. Preparation of the controls for the target cell lines. The indicated volumes are sufficient for 10 replicates ( $100 \mu$ leach).

\begin{tabular}{|c|c|c|c|}
\hline Cell line & hTGFa & hEGF & p38, MEK1/2 inhibitors \\
\hline \multirow{3}{*}{ Ca9-22 } & $\begin{array}{l}\text { Add } 2 \mu \mathrm{l} \mathrm{hTGF}(10 \mu \mathrm{g} / \mathrm{ml}) \text { into } 998 \mu \mathrm{l} \\
\text { starvation media to a concentration of } 20 \\
\mathrm{ng} / \mathrm{ml} \text {. }\end{array}$ & $\begin{array}{l}\text { Add } 2 \mu \mathrm{l} \mathrm{hEGF}(10 \mu \mathrm{g} / \mathrm{ml}) \text { into } 998 \mu \mathrm{l} \\
\text { starvation media to a concentration of } 20 \\
\mathrm{ng} / \mathrm{ml} \text {. }\end{array}$ & \multirow{8}{*}{$\begin{array}{l}\text { Add } 1 \mu \text { l of each inhibitor }(10 \mathrm{mM}) \text { into } 998 \\
\mu l \text { starvation media for a final concentration } \\
\text { of } 10 \mu \mathrm{M} \text { each. }\end{array}$} \\
\hline & & & \\
\hline & $\begin{array}{l}\text { Add } 200 \mu \mathrm{l} \mathrm{hTGF} \mathrm{(} 20 \mathrm{ng} / \mathrm{ml}) \text { into } 800 \mu \mathrm{l} \\
\text { starvation media to a final concentration } \\
\text { of } 4 \mathrm{ng} / \mathrm{ml} \text {. }\end{array}$ & $\begin{array}{l}\text { Add } 300 \mu \mathrm{l} \mathrm{hEGF}(20 \mathrm{ng} / \mathrm{ml}) \text { into } 700 \mu \mathrm{l} \\
\text { starvation media to a final concentration } \\
\text { of } 6 \mathrm{ng} / \mathrm{ml} \text {. }\end{array}$ & \\
\hline \multirow[t]{2}{*}{ H0-1-N-1 } & $\begin{array}{l}\text { Add } 2 \mu \mathrm{l} \mathrm{hTGF}(10 \mu \mathrm{g} / \mathrm{ml}) \text { into } 998 \mu \mathrm{l} \\
\text { starvation media to a concentration of } 20 \\
\mathrm{ng} / \mathrm{ml} \text {. }\end{array}$ & $\begin{array}{l}\text { Add } 2 \mu \mathrm{l} \mathrm{hEGF} \mathrm{(} 10 \mu \mathrm{g} / \mathrm{ml}) \text { into } 998 \mu \mathrm{l} \\
\text { starvation media to a concentration of } 20 \\
\mathrm{ng} / \mathrm{ml} \text {. }\end{array}$ & \\
\hline & $\begin{array}{l}\text { Add } 500 \mu \mathrm{l} \mathrm{hTGF}(20 \mathrm{ng} / \mathrm{ml}) \text { into } 500 \mu \mathrm{l} \\
\text { starvation media to a final concentration of } \\
10 \mathrm{ng} / \mathrm{ml} \text {. }\end{array}$ & $\begin{array}{l}\text { Add } 500 \mu \mathrm{l} \text { hEGF }(20 \mathrm{ng} / \mathrm{ml}) \text { into } 500 \mu \mathrm{l} \\
\text { starvation media to a final concentration of } \\
10 \mathrm{ng} / \mathrm{ml} \text {. }\end{array}$ & \\
\hline \multirow{3}{*}{ HaCat } & $\begin{array}{l}\text { Add } 2 \mu \mathrm{l} \mathrm{hTGF}(10 \mu \mathrm{g} / \mathrm{ml}) \text { into } 998 \mu \mathrm{l} \\
\text { starvation media to a concentration of } 20 \\
\mathrm{ng} / \mathrm{ml} \text {. }\end{array}$ & $\begin{array}{l}\text { Add } 2 \mu \mathrm{l} \mathrm{hEGF}(10 \mu \mathrm{g} / \mathrm{ml}) \text { into } 998 \mu \mathrm{l} \\
\text { starvation media to a concentration of } 20 \\
\mathrm{ng} / \mathrm{ml} \text {. }\end{array}$ & \\
\hline & & & \\
\hline & $\begin{array}{l}\text { Add } 500 \mu \mathrm{l} \mathrm{hTGF} \mathrm{(} 20 \mathrm{ng} / \mathrm{ml}) \text { into } 500 \mu \mathrm{l} \\
\text { starvation media to a final concentration of } \\
10 \mathrm{ng} / \mathrm{ml} \text {. }\end{array}$ & $\begin{array}{l}\text { Add } 250 \mu \mathrm{l} \mathrm{hEGF}(20 \mathrm{ng} / \mathrm{ml}) \text { into } 750 \mu \mathrm{l} \\
\text { starvation media to a final concentration } \\
\text { of } 5 \mathrm{ng} / \mathrm{ml} \text {. }\end{array}$ & \\
\hline
\end{tabular}

\section{Fluorescent labelling with live-compatible dyes. TIMING 25 minutes}

14. Prepare a solution of starvation media with the fluorescent dyes. Add $10 \mu l$ of Hoechst 33342 working solution and optionally $100 \mu \mathrm{l}$ of CellTracker ${ }^{\text {TM }}$ Red CMTPX stock solution int $05 \mathrm{ml}$ starvation media to obtain a final concentration of $2 \mu \mathrm{g} / \mathrm{ml}$ and $2 \mu \mathrm{M}$, respectively.

CRITICAL STEP. Nuclear labelling with Hoechst 33342 stain is crucial for accurate image segmentation and feature extraction with the automated image analysis pipeline presented in this protocol. Labelling of cellular cytoplasm with CellTracker ${ }^{\text {TM }}$ Red CMTPX can be performed to visualize cell boundaries. The concentrations of these dyes may need to be adjusted depending on the selected cell line. When using a different cell line, check that addition of the dyes at the desired concentration does not result in adverse effects in re-epithelialization kinetics.

\section{? TROUBLESHOOTING}

15. Add the labelling solution into a reagent reservoir and carefully aspirate the starvation media from the cells using a vacuum pump without disturbing the cell monolayer.

16. Add $50 \mu \mathrm{l}$ of the solution to each of the wells and incubate at $37^{\circ} \mathrm{C}$ with $5 \% \mathrm{CO}_{2}$ for 20 minutes.

\section{Scratch assay and imaging. TIMING 5-16 hours depending on the cell line}

17. After 20 minutes of incubation with the labelling solution, perform a scratch in the cell monolayers of each well using the HTSScratcher to assure equally sized and reproducible scratches. Place the plate on the platform, slide the plate under the 96-pin array, press the array towards the bottom of the wells, and slide the array from left to right 
3 times. Release pressure from the array and remove the plate from the platform.

CRITICAL STEP. Rinse the array with water followed by ethanol before and after each experiment. ? TROUBLESHOOTING

18. Carefully aspirate the liquid from each well using the vacuum pump and wash the cells with $100 \mu$ PBS. Repeat this step one more time.

19. Aspirate the PBS out and immediately transfer $100 \mu$ l of each sample from the master plate to the corresponding wells in the assay plate using a multichannel pipette.

CRITICAL STEP. Add 100-200 $\mu$ I PBS in the outer wells to avoid differential evaporation of the samples.

\section{? TROUBLESHOOTING}

20. Start image acquisition. For the Ca9-22 or $\mathrm{H} 0-1-\mathrm{N}-1$ cell lines, we recommend programming the microscope to acquire images every 20 minutes for a total of 5 hours. For the HaCat cell line, imaging intervals can be adjusted to 60 minutes for a total of $14-16$ hours.

CRITICAL STEP. The duration of the assay may vary depending on the cell line and can be determined by the time required for the positive control to achieve wound closure.

\section{Image and data Analysis. TIMING 45 minutes}

21. For first time users, download the virtual machine (VM) containing the Kinetic Re-Epithelialization Analysis Pipeline (KREAP) toolbox ${ }^{161}$ as well as the VM playerforyour operating system via https://erasmusmcbioinformatics.github.io/ KREAP/. Import the VM into the player following the step-by-step instructions provided in the manual on the website.

22. Create an index file (.txt) and a compressed plate folder (.zip) containing the acquired images organized in separate folders for each well. Indications on how to create these files are provided at https://erasmusmcbioinformatics. github.io/KREAP/file_formats.

23. Upload the compressed folder and index file into the Galaxy history via the "Get data" tool.

CRITICAL STEP. Set the compressed folder's type to "Zip" in the upload menu.

24. Run the KREAP Image Analysis tool to perform automated image segmentation (Fig. $2 \mathbf{C}$ ). The results are reported in an HTML format and can be downloaded and stored locally ${ }^{161}$. A manual is provided at https://erasmusmcbioinformatics.github.io/KREAP/use_kreap_analysis.

\section{? TROUBLESHOOTING}

25. Run the KREAP Data-Modelling tool to quantify re-epithelialization kinetics (Fig. 1). The results are reported in an HTML format and can be downloaded and stored locally ${ }^{161}$. A manual is provided at https://erasmusmcbioinformatics. github.io/KREAP/use_kreap_modeling.

CRUCIAL STEP. Remember to set the time interval ( $\mathrm{min}$ ) between images in the index file.

\section{? TROUBLESHOOTING}




\section{Timing}

\section{Cell culturing and seeding of cells}

Step 1, growing cells to $70-80 \%$ confluence in $75 \mathrm{~cm}^{2}$ tissue culture flasks: $3-7$ days depending on starting point and doubling time.

Step 2-7, seeding cells in 96-well plates: $\sim 15$ minutes.

Step 8, growing of cells into a cell monolayer: 16 hours (overnight).

\section{Cell starvation}

Step 9-10, cell starvation: 2 hours (might vary depending on the cell line).

\section{Preparation of master plate (can be done in parallel with cell starvation)}

Steps 11-13, preparation of master plate with treatment and control samples: 1.5 hours.

\section{Fluorescent labelling (last 20 minutes of cell starvation)}

Steps 14-16, labelling of cells with fluorescent dyes: 25 minutes.

\section{Scratch assay and imaging}

Step 17, performing scratches in the cell monolayers: less than 1 minute.

Step 18-19, washing cells with PBS and addition of treatments into assay plate: 5-7 minutes.

Step 20, image acquisition: 5-16 hours (may vary depending on the cell line).

\section{Image and data analysis}

Step 21, downloading and importing the KREAP toolbox into the VM player: $\sim 1$ hour (only required once).

Step 22-23, creating and uploading input files for analysis with the KREAP toolbox: 15 minutes.

Step 24, performing automated image analysis of a 96-well plate: 30 minutes.

Step 25, determining re-epithelialization kinetics: less than 1 minute.

\section{? TROUBLESHOOTING}

Troubleshooting advice is found in Table 2. 
Table 2. Troubleshooting.

Step Problem

Possible solution

9 Cells look healthy, but are not confluent after overnight incubation. Increase the seeding density or the time of incubation.

12

No significant difference between the controls

(positive and negative) and untreated cells.

This can result from higher than optimal seeding densities. Decrease the seeding density. Also verify the expiration date and storage conditions of the reagents and replace them if necessary. Avoid repeated freezethaw cycles by storing the reagents in aliquots.

Dim cells can result from incubation with the labelling solution for a too short period of time. Incubate the cells with the dyes for at least 20 minutes. If the problem persists, increase the concentration of the

14 Dim or patchy fluorescence. dye or the exposure time. Patchy staining can result from precipitation of the dye after thawing. Homogenize the dyes stock solutions before preparing the labelling solution. Verify the expiration date and storage conditions of the reagents.

Verify that the pins in the array are clean and move smoothly up and

17 Some cell monolayers were left intact with the HTSScratcher. down. Assure the pins are touching the bottom of the wells when the array is pressed towards the plate.

This can result from uneven sample suspensions. Assure all sample preparations are homogenized. When transferring the samples from the master plate into the assay plate, pipette the treatment solution/ suspensions up and down with the multichannel pipette.

Adjust image segmentation settings in the index file. Generally, the

24

Image analysis pipeline is not performing illumination correction parameter (pixel_block_size) should be set in

optimal image segmentation. a range between 10-20 pixels. Nuclei size typically range between 4 (object_size_min) and 15 (object_size_max) pixels.

This can happen when the re-epithelialization growth curve does not

25

The estimation of the kinetic parameter values for a particular treatment are overestimated. assay.

\section{Anticipated results}

Representative growth curves for each of the target cell lines generated with the measured and modelled number of infiltrating cells over time upon treatment with the positive (i.e. hTGFa and hEGF) and negative (i.e. p38 and MEK1/2 inhibitors) controls at the recommended concentrations are provided in Figure 1. 

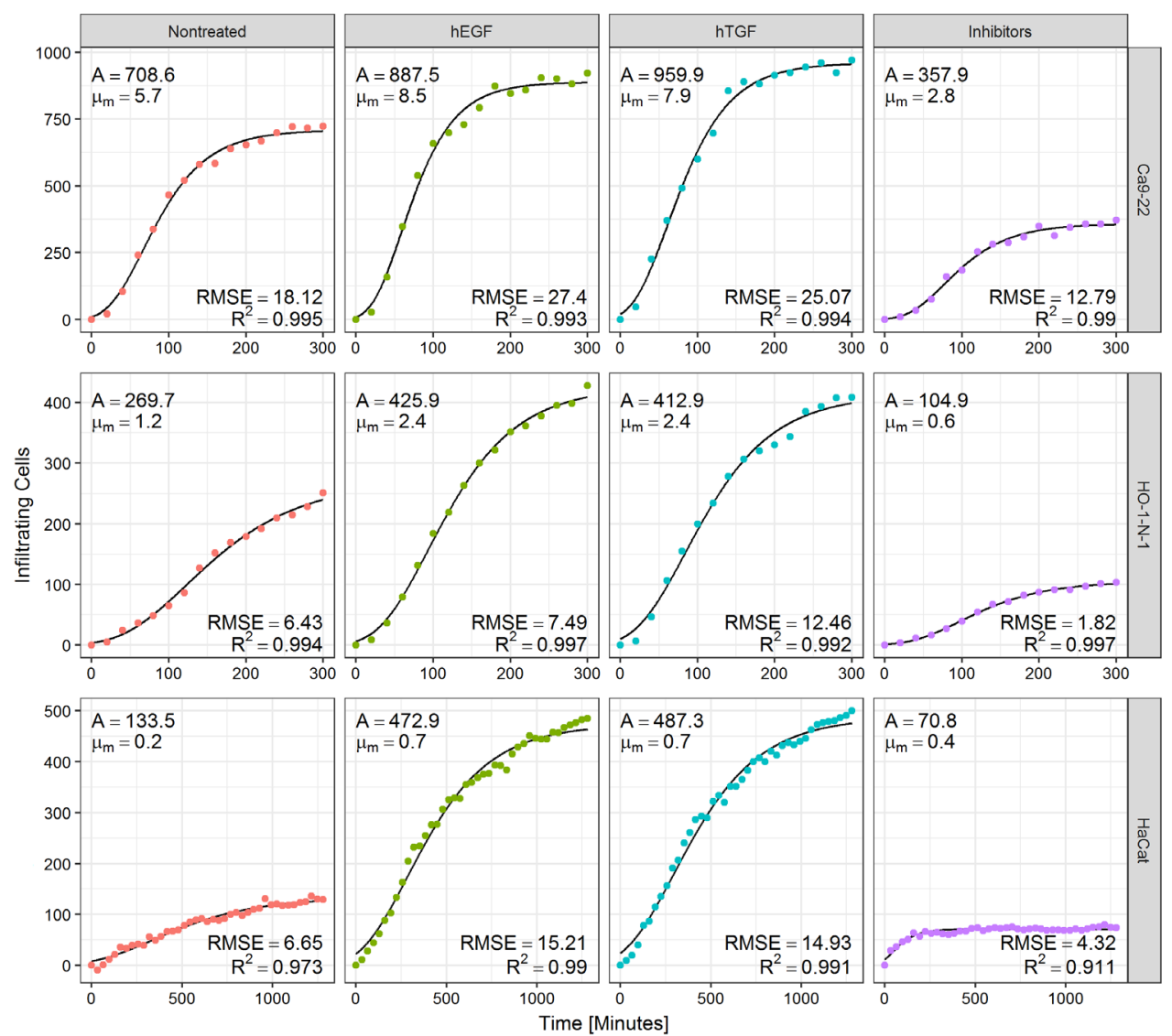

Figure 1. Enumeration of infiltrating cells. The modified Gompertz model (black line) was fitted through the measured data points (colored dots) to estimate the repair rate $\left(\mu_{m}\right)$ and the maximum number of cells inside the scratched area at the plateau of the growth curve (A). Goodness of fit ( $\left.R^{2}\right)$. Root-mean-square error (RMSE).

As an example, we employed this protocol to analyze the influence of Lactobacillus rhamnosus GG (LGG) and Porphyromonas gingivalis (W83) on re-epithelialization of gingival cells. Ca9-22 cells were exposed to three dosages (M0I 5, 50 and 500) of these bacteria and the re-epithelialization kinetics were determined using the KREAP toolbox. Addition of LGG at the intermediate dosage tested led to an increased repair rate $(P=0.03)$ (Fig. 2a) and a significantly higher number of cells inside the scratched area as compared to the non-treated control (Fig. $\mathbf{2 b}$, c). However, higher dosages of LGG resulted in reduced re-epithelialization. This could be attributed to the acidification of the media (down to pH 5) by LGG fermentation products, leading to cell death and suppressed wound closure, which was clearly reflected by the modelled A parameter (i.e. maximum number of cells) (Fig. 2b). In contrast, we obtained a clear negative dosage effect upon exposure of gingival cells to increasing dosages of $P$. gingivalis (Fig. $2 \mathbf{2}, \mathbf{b}$ ). At the higher dosage tested, $P$. gingivalis led to reduced migration of cells into the scratched area, resulting in a significantly lower number of infiltrated cells $(P<0.001)$ as illustrated by the A parameter (Fig. $\mathbf{2 b}$, c). Nonetheless, we also showed that the repair rate $\left(\mu_{\mathrm{m}}\right)$ was not significantly influenced by any of the $P$. gingivalis preparations (Fig. 2a). After introduction of the scratch in the cell monolayer, the cells were not immediately affected by the presence of $P$. gingivalis ( $\mathrm{MOI} 500$ ) and started to migrate into the scratched 
area in an attempt to restore cell-cell contacts. However, during the course of the experiment migration of cells into the scratched area eventually ceased, resulting in an unresolved wound (Fig. 2c) and a low A parameter value.
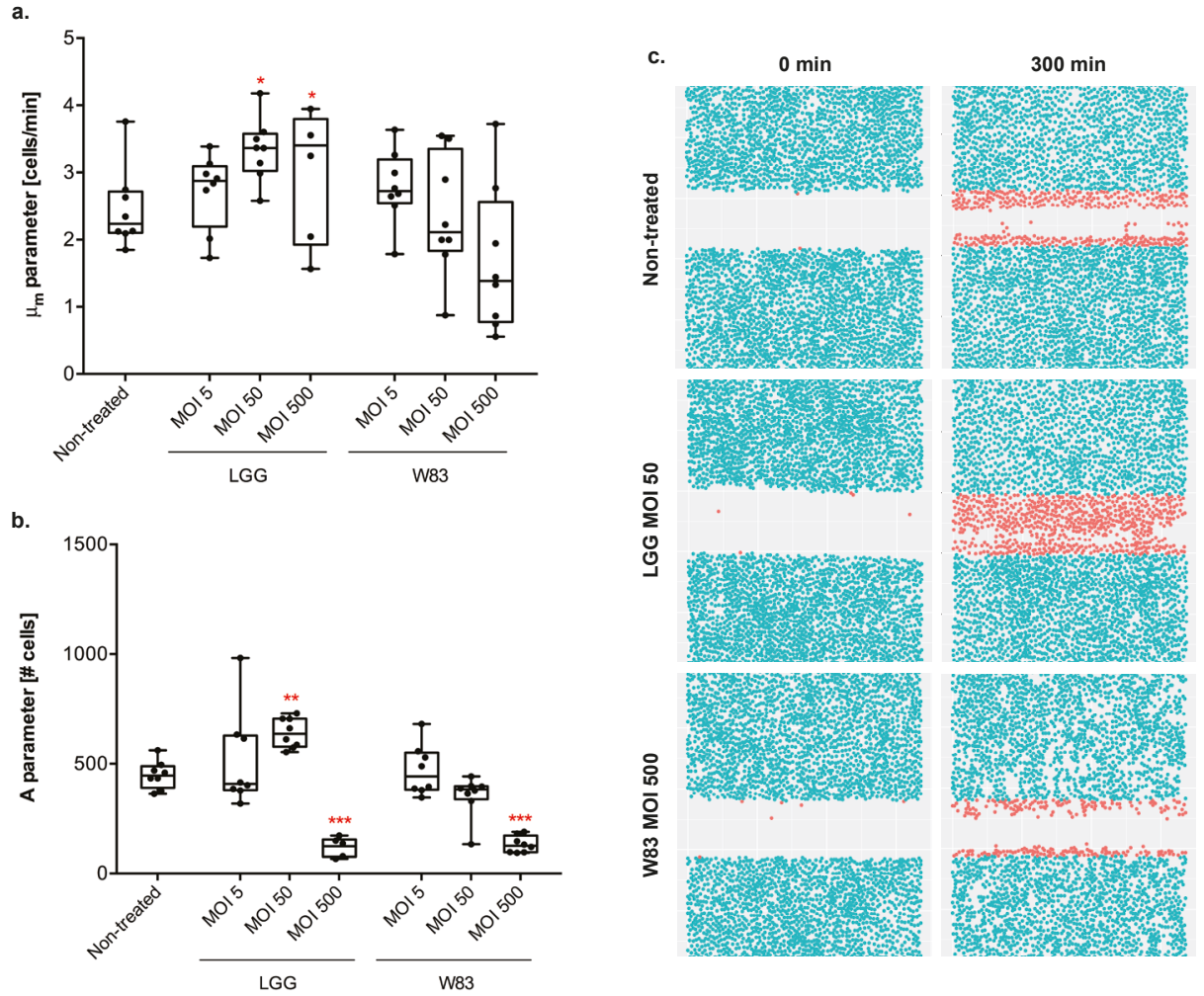

Figure 2. Re-epithelialization kinetics of gingival cells upon exposure to bacterial preparations. (a) Repair rate represented by the $\mu_{\mathrm{m}}$ parameter. (b) Maximum number of cells inside the scratched area represented by the A parameter. Bacterial suspensions were added at an $\mathrm{MOI}$ of 5, 50 and 500. The non-treated control consisted of FCS-free DMEM. Significant differences from the non-treated control were assessed by a one-way ANOVA ( $\mathrm{n} \geq 5$; ${ }^{*}, \mathrm{P}<0.05 ;{ }^{* *}, \mathrm{P}<0.01$; $\left.{ }^{* * *}, \mathrm{P}<0.0001\right)$. (c) Image segmentation output at the start of the experiment $(0 \mathrm{~min})$ and after 5 hours ( $\left.300 \mathrm{~min}\right)$ of incubation with LGG (MOI 50) and P. gingivalis W83 (MOI 500) in comparison with the non-treated control. 


\section{Supplementary data}

a.

\section{Ca9-22 cell line}
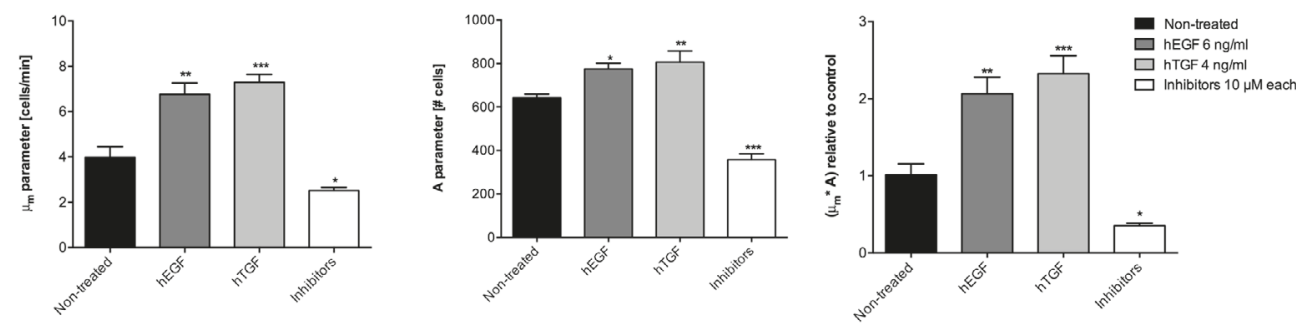

b.

HO-1-N-1 cell line
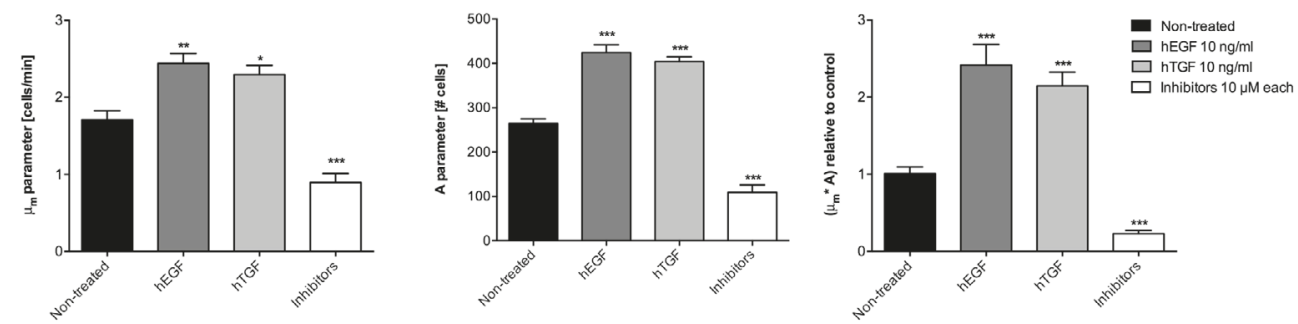

c.

\section{HaCat cell line}
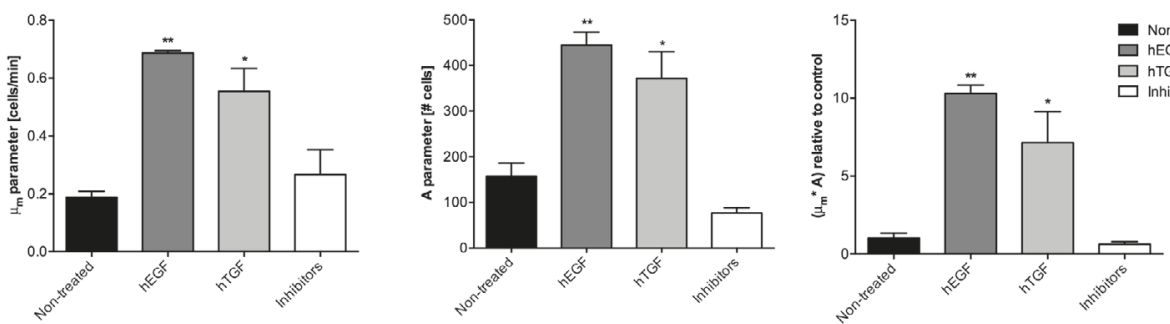

Figure S1. Optimal control concentrations on each target cell line. Human transforming growth factor a (hTGF) and human epidermal growth factor (hEGF) were used as positive controls. Inhibitors of $\mathrm{p} 38$ and MEK1/2 were used in combination as negative control. The repair rate is represented by the $\mu_{\mathrm{m}}$ parameter, whereas the A parameter represents the maximum number of cells inside the scratched area. The overall re-epithelialization performance $\left(\mu_{m}{ }^{*} A\right)$ was calculated relative to the non-treated control. (a) Ca9-22 gingival cell line. (b) H0-1-N-1 buccal cell line. (c) HaCat keratinocyte cell line. Significant differences from the non-treated control were assessed by a one-way ANOVA using a Dunnett's test for multiple comparisons ( $\mathrm{n} \geq$ $\left.3 ;{ }^{*}, P<0.05 ;{ }^{* *}, P<0.01 ;{ }^{* *}, P<0.0001\right)$. 


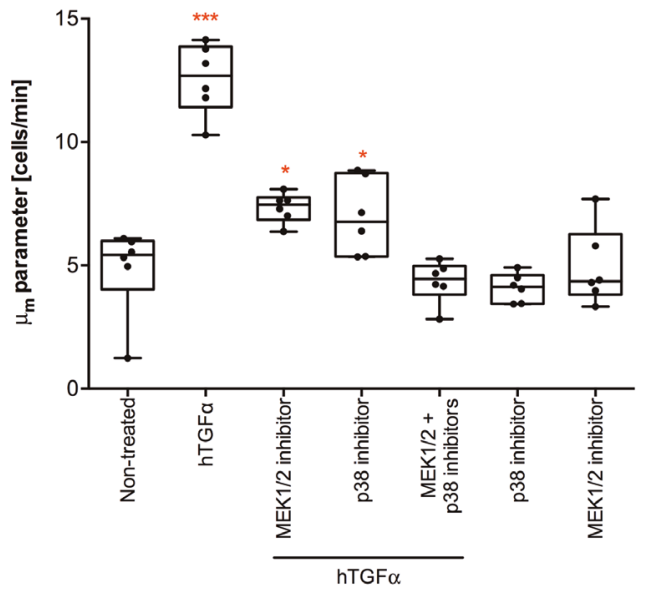

Figure S2. Human transforming growth factor a (hTGFa) promotes re-epithelialization through p38 and MEK1/2 activation. Stimulation of Ca9-22 cells with hTGFa in combination with either inhibitor reduced the repair rate ( $\mu_{m}$ parameter) to an intermediate level. Addition of hTGFa in combination with both inhibitors decreased the repair rate to the level of the non-treated control, indicating that hTGFa can promote both migration and proliferation through activation of $\mathrm{p} 38$ and MEK1/2, respectively. Significant differences from the non-treated control were assessed by a one-way ANOVA using a Dunnett's test for multiple comparisons $\left(n=6 ;{ }^{*}, P<0.05 ;{ }^{* * *}, P<0.0001\right)$. 


\title{
Chapter
}

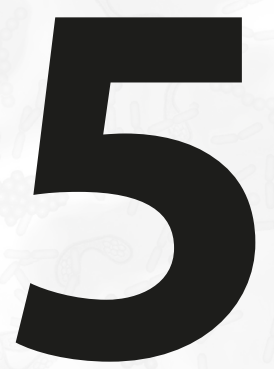

\section{Salivary Metabolite Signature Predicts in vitro Re-Epithelialization Kinetics and is Associated with Gingival Bleeding in Human Volunteers}

\author{
Marcela M. Fernandez-Gutierrez ${ }^{1,2}$, \\ Sultan Imangaliyev ${ }^{1,3,6}$, \\ Andrei Prodan ${ }^{1,4,6}$, \\ Bruno G. Loos ${ }^{1,5}$, \\ Bart J. Keijser ${ }^{1,3,6}$, \\ Michiel Kleerebezem ${ }^{1,2^{*}}$
}

\footnotetext{
'TI Food and Nutrition, Nieuwe Kanaal 9-A, 6709 PA, Wageningen, The Netherlands.

${ }^{2}$ Host-Microbe Interactomics Group, Department of Animal Sciences, Wageningen University \& Research, De Elst 1, 6708 WD, Wageningen, The Netherlands.

${ }^{3}$ TNO Microbiology and Systems Biology, Utrechtseweg 48, 3704 HE, Zeist, The Netherlands. ${ }^{4}$ Department of Oral Biochemistry, Academic Centre for Dentistry Amsterdam (ACTA),

University of Amsterdam and Vrije Universiteit Amsterdam, Gustav Mahlerlaan 3004, 1081 LA,

Amsterdam, The Netherlands.

${ }^{5}$ Department of Periodontology Academic Centre for Dentistry Amsterdam (ACTA), University of Amsterdam and Vrije Universiteit Amsterdam, Gustav Mahlerlaan 3004, 1081 LA, Amsterdam, The Netherlands.

${ }^{6}$ Department of Preventive Dentistry, Academic Centre for Dentistry Amsterdam (ACTA), University of Amsterdam and Vrije Universiteit Amsterdam, Gustav Mahlerlaan 3004, 1081 LA,

Amsterdam, The Netherlands.
} 
Several proteins and peptides in human saliva have been shown to have the capacity to stimulate wound repair in the oral cavity. However, the contribution of the salivary chemical composition to the healing process remains unexplored. In this study, we measured the re-epithelialization kinetics of gingival epithelial cells in vitro upon treatment with a subset of 63 unstimulated saliva samples collected during an experimental gingivitis study conducted with 61 healthy individuals. The metabolite profiles of these saliva samples were used to perform an elastic net regression with stability selection to identify a set of metabolites that were associated with the re-epithelialization kinetics observed in the in vitro model. The metabolite signature proved to be specific and could predict the re-epithelialization capacity of the remaining 242 saliva samples collected during the clinical study. Enhanced re-epithelialization by saliva was associated with higher concentrations of several metabolites, including plasmalogens, diacylglycerol and amino acid derivatives, which have been proposed to reflect host-microbe interactions, cell damage and/or inflammation. In line with these findings, the predicted re-epithelialization capacity of the unstimulated saliva samples was positively associated with the percentage of gingival bleeding, a clinical parameter that is considered to reflect the inflammatory state of the periodontium. The saliva samples derived from most of the individuals displayed a low-level of variation with respect to their capacity to stimulate re-epithelialization during the experimental gingivitis challenge. However, a subset of individuals displayed considerable variation over time, which was associated with increased gingival bleeding (responsiveness) during the challenge period. This finding implies that individuals with a higher instability of the salivary metabolite signature identified here, may have an elevated risk to develop gingivitis. Unravelling the mechanisms by which the combination of these metabolites can stimulate wound repair may offer novel avenues towards therapeutic approaches in patients with impaired wound healing capacity. 


\section{Introduction}

The oral mucosa is exposed to frequent mechanical abrasion and chemical stress that result from daily activities such as eating, drinking, speaking and chewing. Oral wound repair is distinguished from dermal healing by its higher repair rate and lack of scar formation ${ }^{74}$. The rapid wound repair observed in the oral cavity has been attributed to several factors. First, the oral epithelial cells have a higher turnover rate than skin cells ${ }^{8}$, which allows a faster repair of the oral mucosa. Second, there is a better microcirculation in the oral mucosa than in the skin, facilitating faster recruitment of immune cells, growth factors and nutrients to the site of injury, and more effective removal of phagocytized bacteria and cell debris ${ }^{29}$. Finally, the presence of saliva provides a humid environment that promotes re-epithelialization through faster cell migration ${ }^{164}$. Moreover, human saliva contains a variety of proteins and peptides that have been shown to promote wound repair. For example, epidermal growth factor (EGF) ${ }^{165}$ and transforming growth factor alpha (TGFa) ${ }^{166}$ play an important role during oral wound repair by activation of several signalling cascades that lead to migration, differentiation, and proliferation of cells. Similarly, vascular endothelial growth factor (VEGF) is involved in re-epithelialization, extracellular matrix regulation and angiogenesis, which are critical processes that take place during wound repaii ${ }^{167}$. Certain antimicrobial peptides present in saliva such as $\beta$-defensins and cathelicidin have also been reported to have growth stimulating properties in keratinocytes and lung cells ${ }^{168,169}$. In particular, histatin (Hst) 1 and Hst2, primarily recognized for their antimicrobial activity in the oral cavity, have been identified as potent stimulators of cell migration via activation of the ERK $1 / 2$ pathway in oral cells ${ }^{75}$.

The role of individual salivary proteins or peptides on maintenance of oral health has been extensively studied ${ }^{28}$. Nevertheless, saliva is also a rich source of small molecules $(<2,000 \mathrm{Da})$, which unlike the salivary proteome, have not been characterized in detail. Recently, a total 853 human salivary metabolites were identified and annotated using a multiplatform approach in combination with computer aided literature-mining ${ }^{23}$. This metabolite collection complemented the work of the Human Metabolome Database (HMDB) (http://www.hmdb.ca/) which provides the most up to date and comprehensive library of the human metabolome ${ }^{32}$.

In a previous study, we examined the changes in the salivary metabolome of 61 healthy adults at 5 timepoints during an experimental gingivitis challenge intervention ${ }^{170}$. The challenge intervention required the volunteers to refrain from any form of oral hygiene for two weeks, resulting in plaque accumulation and induction of mild inflammation. Using nontargeted metabolomics, we were able to identify 497 metabolites that were quantified in a total of 305 unstimulated saliva samples collected during the challenge period of the study. Here we identified a metabolite signature composed of ten compounds, which relative concentrations are able to predict the effect of saliva on gingival re-epithelialization kinetics in a recently developed in vitro model system ${ }^{155}$. Several of the compounds of the signature are associated with increased host-microbe interactions, cell damage and/or inflammation, which was reflected by a positive association between the predicted gingival re-epithelialization capacity elicited by the saliva samples and the inflammatory state of the periodontal tissues. Finally, instability of the salivary metabolite signature, appeared to be correlated with elevated responsiveness in the experimental gingivitis model. 


\section{Materials and Methods}

\section{Study population and design}

A challenge intervention, randomized, blinded study was carried out in the Academic Centre for Dentistry Amsterdam (ACTA) within the framework of the Top Institute of Food and Nutrition (TIFN) project "An exploratory study on the dynamic (microbial, biochemical and immunological) interactions of the oral ecosystem during induction of mild gingival inflammation". The study population consisted of 61 systemically healthy adults ( 21 males, 40 females), between 18 and 55 years of age, with no apparent periodontitis or dental caries. Inclusion and exclusion criteria were previously described by Prodan et. al. ${ }^{170}$. The study consisted of three phases: baseline, challenge intervention, and resolution phase. The baseline phase consisted of two weeks (Day -14 to Day 0 ) in which the volunteers of the treatment group $(n=20)$ were requested to start taking six doses of erythritol per day ( $2 \mathrm{~g} /$ tablet $)$ for the entire duration of the study ( 5 weeks). Erythritol is a non-caloric polyol that is used as sugar substitute and has been shown to reduce plaque accumulation, limit growth of Streptococcus mutans, and lower caries rates in vivo ${ }^{171-173}$. The challenge intervention was based on the experimental gingivitis protocol developed by Löe et. al. ${ }^{174}$ and encompasses a two-week period (Day 0 to Day 14) in which all participants were asked to refrain from any form of oral hygiene (i.e. tooth brushing, flossing, chewing gum, mouth wash), resulting in plaque accumulation and induction of mild gingival inflammation. This was followed by a resolution phase of one week (Day 14 - Day 21) in which the volunteers reinitiated standardized oral hygiene practices to restore normal oral homeostasis.

\section{Ethical considerations}

The study protocol was reviewed and approved by the Medical Ethics Committee of the Vrije Universiteit Medical Centre (2014_505) and registered at the Central Committee on Research Involving Human Subjects (CCM0). The study was conducted according to the Declaration of Helsinki ${ }^{175}$ of the World Medical Association. All volunteers signed an informed consent form.

\section{Unstimulated saliva collection}

Unstimulated saliva samples were collected at seven timepoints across the study (Day -14, Day 0, Day 2, Day 5, Day 9, Day 14 and Day 21). Sample collection occurred between 9.00 and $12.00 \mathrm{AM}$. Volunteers were instructed not to eat or drink two hours prior to the appointment. During sample collection, volunteers were told to accumulate saliva on the floor of their mouth, without facial or tongue movements, and spit every 30 seconds during a period of 5 minutes into a sterile polypropylene tube that was kept on ice. Samples were centrifuged $\left(10,000 \mathrm{xg}, 10\right.$ minutes at $\left.4^{\circ} \mathrm{C}\right)$ to remove epithelial cell debris, bacteria, and food residues and were then stored at $-80^{\circ} \mathrm{C}$. Total protein content was determined using a Pierce BCA Protein Assay Kit (Thermo Fisher Scientific, MA, USA) as described in ${ }^{176}$.

\section{Metabolomics analysis}

Non-targeted metabolite profiling of the saliva samples was outsourced to Metabolon ${ }^{\circledast}$ (Durham, NC, USA) and processed as described previously ${ }^{170}$. Briefly, metabolite extracts were divided into five aliquots: two for analysis by two separate reverse phase ultrahigh performance liquid chromatography-tandem mass spectroscopy (RP/UPLC-MS/MS) methods 
with positive ion mode electrospray ionization (ESI), one for analysis by RP/UPLC-MS/MS with negative ion mode ESI, one for analysis by hydrophilic-interaction chromatography (HILIC)/UPLC-MS/MS with negative ion mode ESI, and one sample was reserved as backup. All methods used an ACQUITY UPLC System (Waters Corporation, MA, USA) and a Q-Exactive ${ }^{\text {TM }}$ high-resolution, accurate-mass spectrometer (Thermo Fisher Scientific) interfaced with a heated electrospray ionization (HESI-II) source and Orbitrap mass analyzer operated at 35,000 mass resolution. Extraction of raw data, quality control processing and identification of peaks were performed using Metabolon's hardware and software. Identification of peaks was carried out by comparison with the Metabolon's reference library, which contains the retention time/index (RI), mass to charge ratio $(\mathrm{m} / \mathrm{z})$, and chromatographic data on all molecules present in the library. Inter-day instrument tuning differences were corrected for each compound by registering the medians equal to one in every run and normalizing each data point proportionally. Normalized peak areas were scaled by the median value of each compound. Metabolomics analysis was performed on the five timepoints (Day 0, Day 2, Day 5, Day 9 and Day 14) corresponding to the challenge intervention phase of the study (see also Fig. 1a). The metabolite data is provided as supplementary material in Prodan et. al. $(2016)^{170}$.

\section{Assessment of bleeding on marginal probing (BOMP)}

Assessment of bleeding on marginal probing (BOMP) was performed as previously described by Oliveira et. al. ${ }^{177}$ at the baseline of the study (Day -14 and Day 0), at the peak of the challenge intervention (Day 14) and after the resolution period (Day 21). The teeth with exception of third molars were clinically examined at six sites per tooth (disto-vestibular, vestibular, mesio-vestibular, disto-lingual, lingual and mesio-lingual) in two contralateral quadrants (i.e. one upper and one lower). The number of gingival units that bled upon marginal probing within 30 seconds was recorded with scores from 0 to 2 ( 0 = no bleeding, 1 = pinprick, 2 = excessive bleeding). Gingival bleeding was expressed as a percentage of sites that bled upon probing and the response to the challenge was expressed by calculating the difference between the percentage of gingival bleeding at the end (Day 14 ) and at the beginning (Day 0 ) of the challenge ( $\triangle B O M P \%$ ).

\section{Cell line maintenance}

The human epithelial gingival cell line, Ca9-22 (JCRB0625), was purchased from the National Institute of Biomedical Innovation JCRB Cell Bank (Osaka, Japan). Ca9-22 cells were cultured in Dulbecco's Modified Eagle Medium (DMEM) containing Glutamax (Invitrogen, Paisley, UK) and supplemented with 10\% fetal calf serum (FCS), 100U/mL penicillin and $100 \mu \mathrm{g} / \mathrm{mL}$ streptomycin containing $5 \% \mathrm{CO}_{2}$. The cells were passaged every three to four days

\section{Scratch assay and re-epithelialization kinetics modelling}

Scratch assays and modelling of re-epithelialization kinetics were performed as described in ${ }^{155}$. Cells were seeded in 96well plates (BD Falcon ${ }^{\mathrm{TM}}$, Corning, NY, USA) at a density of $3.5 \times 10^{4}$ cells/well and incubated overnight until a confluent cell monolayer was formed. The next day, cells were starved in FCS-free DMEM for 2 hours. During the last 20 minutes of starvation, the cytoplasm and nuclei were labelled with $2 \mu \mathrm{M}$ CellTracker ${ }^{\mathrm{TM}}$ Red CMTPX (Molecular Probes, OR, USA) and $2 \mu \mathrm{g} / \mathrm{ml}$ Hoechst 33342 (Molecular Probes), respectively. Longitudinal scratches $(1.5 \times 4 \mathrm{~mm})$ were performed in the cell monolayers of the multi-well plate using the HTSScratcher (Peira, Antwerpen, BE). Wells were washed twice with phosphate-buffered saline (PBS), after which the treatments were immediately added into the wells. Unstimulated saliva 
samples were tested in duplicate in a 1:4 ratio (unstimulated saliva : FCS-free DMEM). As positive control of cell migration and proliferation, $4 \mathrm{ng} / \mathrm{ml}$ human transforming growth factor alpha (TGFa; R\&D Systems, MN, USA). A combination of p38 (SB203580; Cell Signaling Technology, MA, USA) and MEK1/2 (U0126, Cell Signaling Technology) inhibitors was used as negative control at a concentration of $10 \mu \mathrm{M}$ each. Bright-field and fluorescent images ( $577 \mathrm{~nm}$ and $350 \mathrm{~nm}$ ) of the same frame of each well were acquired every 20 minutes during a period of 5 hours with the BD Pathway 855 Bioimaging System (BD Biosciences, $\mathrm{CA}$, USA) using a $4 \mathrm{x}$ objective under controlled conditions $\left(37^{\circ} \mathrm{C}\right.$ and $\left.5 \% \mathrm{CO}_{2}\right)$. Image segmentation and feature extraction was performed using CellProfiler 2.1.1. (http://www.cellprofiler.org/), and FCS Express 4 Plus (De Novo Software, CA, USA) was used to record the number of cells infiltrating into the scratched area over time. The enumeration of infiltrating cells was modelled using the modified Gompertz function ${ }^{105}$ to extract kinetic wound-repair parameters. The $\mu_{\mathrm{m}}$ parameter represents the repair rate (cells minute ${ }^{-1}$ ) and the A parameter represents the maximum number of cells inside the scratched area at the plateau phase of the repair curve. Previous studies have shown that the third parameter obtained by kinetic modelling ( $\lambda$, representing the point of infliction or lag-phase) had limited biological relevance when using the Ca9-22 gingival cells as the migration process in this particular cell line starts very shortly after the scratch is introduced ${ }^{155}$. In addition, the overall wound repair-performance was assessed by the $\left(\mu_{m}{ }^{*} A\right)$ value that is expressed relative to the non-treated control (FCS-free DMEM). Treatments of which the wound-repair curve could be modelled with a goodness of fit $\left(R^{2}\right)$ lower than 0.9 , were discarded from further analysis.

\section{Elastic net regression and stability selection method}

Elastic net regression ${ }^{178}$ with stability selection ${ }^{179}$ was performed to select a set of features (i.e. metabolites) that were related to re-epithelialization kinetics. The elastic net regression with stability selection was implemented in Python using the scikitlearn package v.0.16.1 ${ }^{180}$. The elastic net balancing parameter was set at 0.3 to 0.8 in 20 steps, whereas the alpha factor parameter ranged from $10^{-2}$ to $10^{+2}$ in 150 steps. The $\mu_{\mathrm{m}}$ and $\mathrm{A}$ parameter values as well as the performance value $\left(\mu_{\mathrm{m}}{ }^{*} \mathrm{~A}\right)$ obtained for a total of 63 unstimulated saliva samples were used to train the model using different parameter sets. The features were selected based on their stability after 100 runs of feature selection model on random data shuffles using the stability selection procedure. Model hyperparameters were estimated using an exhaustive grid search within a 5-fold crossvalidation procedure on $80 \%$ of the training dataset, and the model's generalization error was assessed on the remaining $20 \%$ test dataset. The performance measure used for a regression task is a Normalized Root Mean Square Error (NRMSE). Missing metabolite values were assumed to be below the detection limits, and were imputed with the minimum detected value for that metabolite across all samples. Metabolites with more than $25 \%$ missing measurements were removed from the data set. The filtered metabolite measurements were scaled by removing the mean value of each metabolite (zero-mean) and then divided by the standard deviation. Re-epithelialization parameter values were zero-mean scaled. The selected features were used to predict the in vitro re-epithelialization kinetics $\left(\mu_{m}{ }^{*} A\right)$ of the remaining 242 unstimulated saliva samples.

\section{Other statistical analyses}

Statistical significance tests and correlation analyses were performed in GraphPad Prism version 6.0 (GraphPad Software, (A, USA). Linear and multiple linear regressions were performed in R. Heatmaps with hierarchical clustering were created in $\mathrm{R}$ using the heatmap.2 function of the gplots package version 3.0.1 ${ }^{181}$. To create the heatmaps, the normalized metabolite values were scaled by subtracting the mean of the metabolite in the given dataset and then divided by the standard deviation. 


\section{Results}

\section{Stimulatory capacity of saliva on re-epithelialization is highly variable among individuals and is potentially influenced by their metabolome}

In a previous study, we evaluated the changes that occur in the salivary metabolome of healthy adults during the development of plaque-induced gingivitis ${ }^{170}$. The study comprised three phases: a baseline, a challenge intervention, and a resolution phase (Fig. 1a). The baseline consisted of two weeks in which the volunteers assigned to the treatment group were instructed to start taking six doses of erythritol tablets per day (12 g/day) until the end of the study (end of week 5). During the challenge intervention, all the volunteers enrolled in the study were asked to refrain from any form of oral hygiene for two weeks, resulting in accumulation of dental plaque and induction of mild gingival inflammation (i.e. gingivitis). After the two-week challenge intervention, the volunteers resumed standardized oral hygiene and were monitored for one more week during the resolution phase. Daily intake of erythritol had no significant effects on the salivary metabolome ${ }^{170}$ or its capacity to promote wound repair (Fig. S1). Additionally, neither intake of erythritol nor the experimental gingivitis challenge had a significant effect on the levels of salivary proteins measured, buffered $\mathrm{pH}$, flow rate, and protein content, suggesting that the salivary secretions were relatively stable regardless of the changes occurring during the challenge $\mathrm{e}^{170}$.

To investigate if changes in the metabolite profiles could alter salivary wound repair capacity during different stages of the challenge intervention, we tested the effect of the collected unstimulated saliva samples on the re-epithelialization kinetics of gingival epithelial cells (i.e. Ca9-22 cell line). Confluent cell monolayers were scratched and treated with unstimulated saliva samples obtained from 9 randomly selected participants at the 7 timepoints during the study (Day -14 to Day 21; Fig. 1b). Live imaging, followed by modelling of the re-epithelialization curves were used to obtain kinetic parameters ( $\mu_{\mathrm{m}}$ and $\mathrm{A}$; see materials and methods section for details) that quantitatively describe wound-repair. The overall impact on re-epithelialization kinetics of the treatment with specific saliva samples was assessed by the re-epithelialization performance value $\left(\mu_{m}{ }^{*} A\right)$, which was expressed relative to the untreated control within the same assay run ${ }^{155}$. Even though the effect of unstimulated saliva on oral re-epithelialization kinetics was highly variable among individuals (Fig. 1b), the saliva samples derived from most individuals resulted in significantly enhanced re-epithelialization kinetics when compared to the non-treated control, although the stimulatory effect observed using samples obtained from participants 55 and 57 was not significant relative to the untreated control. The stimulatory effect observed on re-epithelialization elicited by the saliva samples from specific individuals did not show a consistent change during the time-course of the study (Fig $\mathbf{1 b}$ and Fig. 1c), indicating that the stimulatory capacity of saliva was not significantly influenced by the experimental gingivitis challenge. In addition, no significant association was found between total protein content and the capacity of the saliva samples to promote gingival re-epithelialization (Fig. S2). 
a.

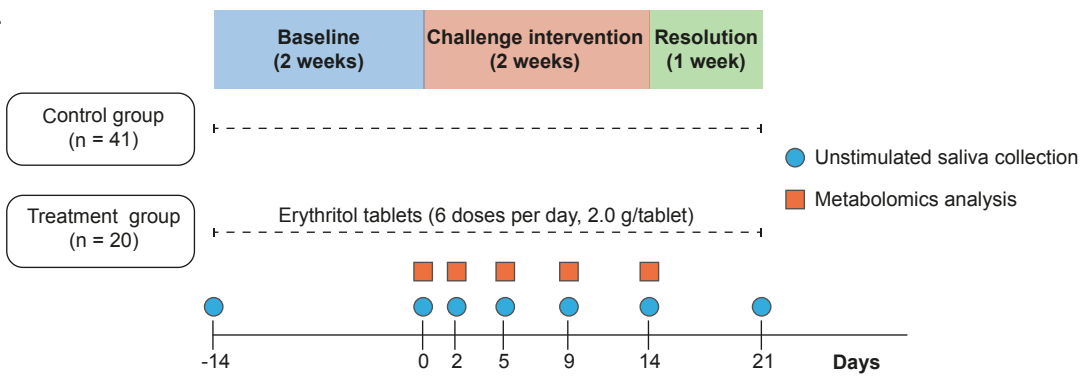

b.

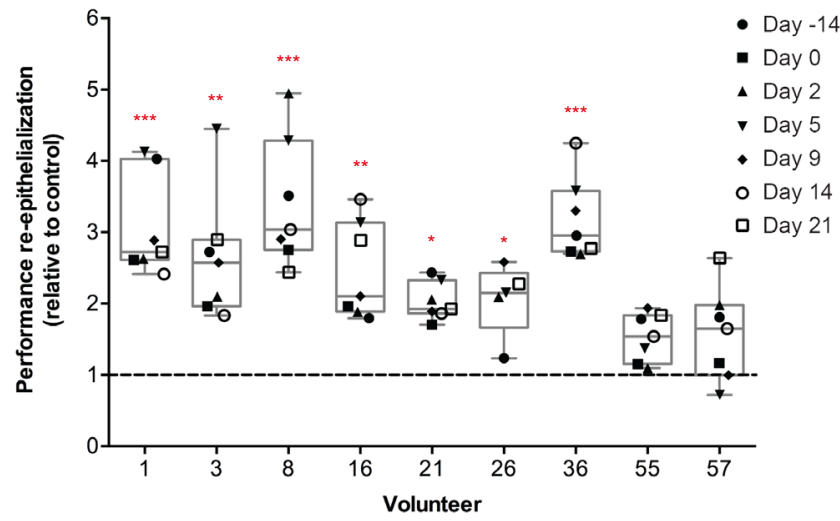

c.

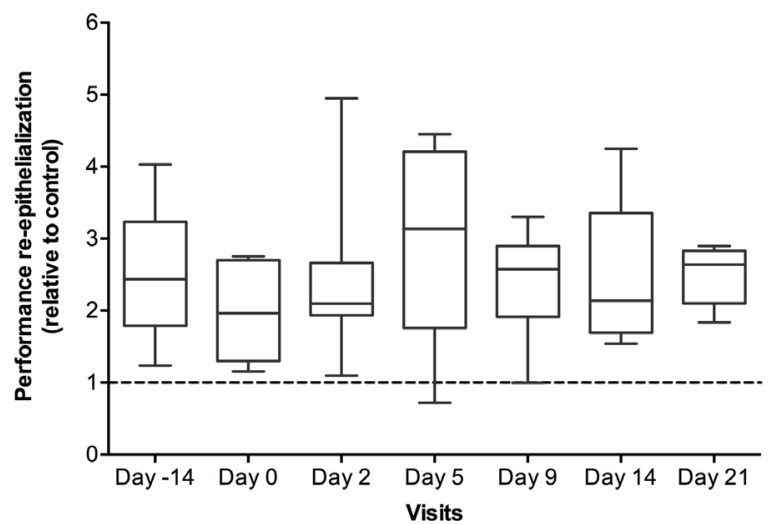

Figure 1. Study design and saliva effect on re-epithelialization kinetics. (a) Design of the challenge intervention, randomized study, adapted from Prodan et al. ${ }^{170}$. (b) Effect of the selected unstimulated saliva on re-epithelialization kinetics. Volunteers 1, 3, 16, 21, 55 and 57 corresponded to the control group. Volunteers 8, 26 and 36 corresponded to the treatment group. Dashed line represents the non-treated control. (c) Re-epithelialization capacity of unstimulated saliva collected at different timepoints of the challenge intervention study. Significant differences from the non-treated control were assessed by a one-way ANOVA followed by a Dunnett's test for multiple comparisons $\left({ }^{*}, \mathrm{P}<0.05 ;{ }^{* *}, \mathrm{P}<0.001\right.$; ${ }^{* * *}$, $P<0.0001)$. 


\section{Metabolite signature in saliva is associated with re-epithelialization kinetics in oral cells}

To investigate whether the chemical composition of saliva influences the differential re-epithelialization capacity observed between samples, we performed elastic net regressions ${ }^{178}$ with stability selection ${ }^{179}$ to identify a set of metabolites that collectively were associated with the observed oral re-epithelialization kinetics. To increase the power of this analysis, the kinetic parameters determined for the saliva samples collected during the challenge intervention period (Day 0 to Day 14, see Fig. 1a) was expanded with 20 randomly selected samples (Table S1; different individuals and different timepoints) for which metabolome data was available (i.e. excluding samples collected at Day -14 and 21). The filtered and scaled metabolite dataset (see Materials and Methods for more details) together with each of the scaled re-epithelialization parameter values, was employed in the elastic net regressions. The best fit of the model $\left(R^{2}=0.54\right)$ was obtained with the performance value $\left(\mu_{\mathrm{m}}{ }^{*} \mathrm{~A}\right)$ (Fig. 2a). In addition, based on this parameter, the model also displayed the lowest normalized root mean-square-error (NRMSE $=16.58$ ) when compared with the actual measurement ${ }^{182}$. Therefore, the overall reepithelialization performance values $\left(\mu_{m}{ }^{*} A\right)$ were used for elastic net regression in combination with the stability selection method ${ }^{179}$ to identify a corresponding salivary metabolite signature (Table 1). Notably, the use of the $\mu_{\mathrm{m}}$ parameter in the elastic net regression also resulted in a very good fit $\left(R^{2}=0.52\right.$, NRMSE $=20.19$, see Fig. $\mathbf{S 3}$ ) and led to the selection of a metabolite set that displayed overlap with the signature identified using the performance value $\left(\mu_{m}{ }^{*} A\right)$, including the plasmalogen lipids and the 0 -sulfo-L-tyrosine (Table $\mathbf{S 2}$ ). This finding suggests that the $\mu_{\mathrm{m}}$ parameter may has a greater contribution to the model than the A parameter.

All the metabolites in the identified signature had a high stability $(>0.6)$, indicating that the metabolites were selected in more than $60 \%$ of the subsampling iterations $(n=100)$ performed. In particular, 0 -sulfo-L-tyrosine had a very high stability and was selected in $91 \%$ of the iterations. Due to the high stability of 0 -sulfo-L-tyrosine in the elastic net regression, we evaluated the potential of this single metabolite as a biomarker of saliva-induced re-epithelialization by performing a linear regression to model the relationship between the concentration of this metabolite and the reepithelialization kinetics measured in the in vitro assay. The fit of this single metabolite model $\left(R^{2}=0.23, P=4.21 \times 10^{-5}\right)$ was much lower than the fit obtained by a multiple linear regression that included all the metabolites in the identified signature $\left(R^{2}=0.74, P=4.80 \times 10^{-14}\right)$, while analysis of variance between the two models showed that the addition of all the metabolites in the signature significantly contributed to the observed re-epithelialization kinetics $(F=2.01$, $\left.P=2 \times 10^{-11}\right)$. These observations strongly support the higher biomarker-value of the metabolite signature as compared to the best-performing single metabolite within the signature.

Besides choline phosphate, all other selected metabolites in the elastic net regression had a positive average weight, indicating that increasing concentrations of these metabolites resulted in enhanced re-epithelialization kinetics. A hierarchical clustering of the relative concentrations of the metabolites in the identified salivary signature, revealed two main clusters in the saliva sample set used to generate the model (Fig. 2b). In agreement with the elastic net regression analysis, the majority of the saliva samples in the upper cluster (I) contained relatively high concentrations of the signature metabolites that resulted in high re-epithelialization kinetics in gingival cells. Only for choline phosphate, the relation between its relative concentration and the re-epithelialization kinetics was inversed. Conversely, the lower cluster (II) grouped saliva samples with relative low concentration of most signature metabolites, which induced lower re-epithelialization kinetics. This cluster differentiation is further substantiated by the highly significant difference in re- 
epithelialization kinetics between the sample groups $(P<0.0001)$ (Fig. 2c). Notably, similar hierarchical clustering using a total of 70 heatmaps generated using randomly selected metabolites from the dataset (Fig. S3) failed to separate the saliva samples into two distinctive clusters with distinct re-epithelialization kinetics, underpinning the specificity of the metabolite signature identified.

Table 1. Metabolite signature. Elastic net regression with stability selection was performed to select a set of metabolites that were associated to re-epithelialization kinetics $\left(\mu_{m}^{*} A\right)$.

\begin{tabular}{|c|c|c|c|}
\hline Metabolite name & Description & Stability & Average Weight \\
\hline 1-(1-enyl-palmitoyl)-2-arachidonoyl-GPE (P-16:0/20:4) & Plasmalogen & 0.69 & 0.14 \\
\hline 1-(1-enyl-palmitoyl)-2-oleoyl-GPC (P-16:0/18:1) & Plasmalogen & 0.67 & 0.09 \\
\hline 1-oleoyl-2-linoleoyl-glycerol (18:1/18:2) & Diacylglycerol & 0.75 & 0.11 \\
\hline 5-aminovalerate & Lysine metabolism & 0.67 & 0.07 \\
\hline choline phosphate & Phospholipid metabolism & 0.63 & -0.17 \\
\hline imidazole lactate & Histidine metabolism & 0.88 & 0.17 \\
\hline Indolelactate & Tryptophan metabolism & 0.72 & 0.10 \\
\hline $\mathrm{N}$-acetylproline & Urea cycle; arginine and proline metabolism & 0.79 & 0.14 \\
\hline 0-sulfo-L-tyrosine & Chemical & 0.91 & 0.21 \\
\hline phosphoenolpyruvate (PEP) & $\begin{array}{l}\text { Glycolysis, gluconeogenesis and pyruvate } \\
\text { metabolism }\end{array}$ & 0.73 & 0.14 \\
\hline
\end{tabular}

\section{Predicting in vitro oral re-epithelialization kinetics using the salivary metabolite signature}

To validate the predictive potential of the metabolite signature, we used it to predict the re-epithelialization capacity of the remaining 242 unstimulated saliva samples collected during the challenge intervention period of the study ( $n=305$ ) (Fig 1a). Subsequently, we selected previously untested saliva samples that were predicted to have among the highest and lowest stimulatory capacity ( $n=11$ in each group) in the re-epithelialization assay. The re-epithelialization kinetics of gingival cells stimulated with these samples was determined and showed that the metabolite signature could successfully predict the relative re-epithelialization capacity of the saliva samples (Fig 3). Similar to the training sample set, the metabolite signature separated the samples in two distinct clusters (I and II; Fig. 3a) that induced significantly higher (cluster I) and lower (cluster II) wound repair kinetics ( $P=0.02$ ) (Fig $\mathbf{3 b}$ ). Moreover, Pearson correlation analysis corroborated a significant association between the observed and predicted kinetics of re-epithelialization $(r=0.51, P=0.015)$. Taken together, the combination of several biomarkers into the metabolite signature presented in this study has a powerful predictive potential of the effect of the saliva in oral re-epithelialization. 
a.
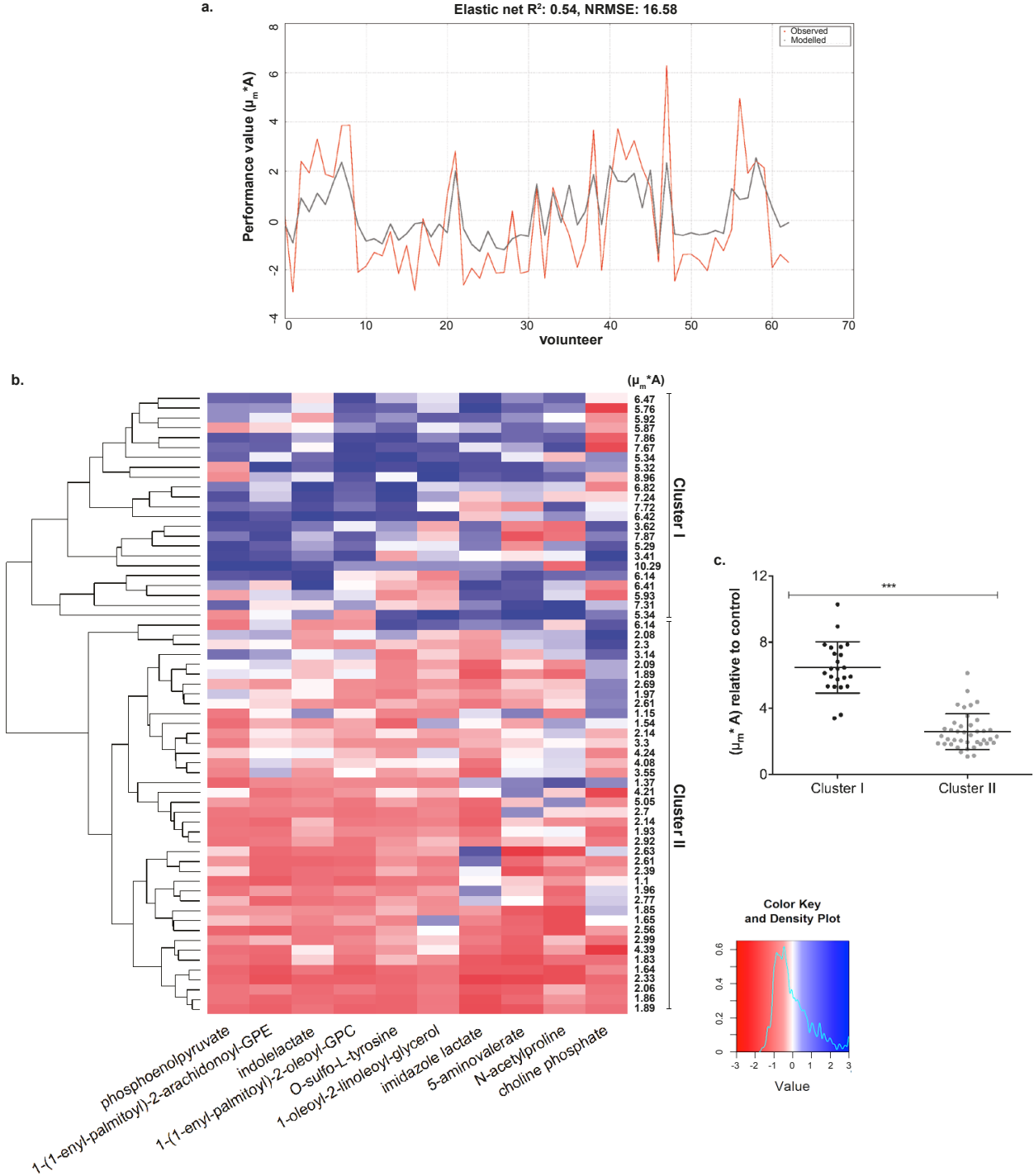

Figure 2. Salivary metabolite signature related to observed re-epithelialization kinetics. (a) Performance of the elastic net regression through the $\left(\mu_{m}{ }^{*} A\right)$ parameter values. (b) Hierarchical clustering heatmap displaying relative metabolite concentrations of the signature and observed re-epithelialization kinetic values $\left(\mu_{m}{ }^{*} A\right)$ for the training dataset. (c) Re-epithelialization performance induced by saliva samples of clusters I and II derived from the hierarchical clustering. Significant difference was assessed by a two-tailed t-test (***, $P<0.0001$ ). 

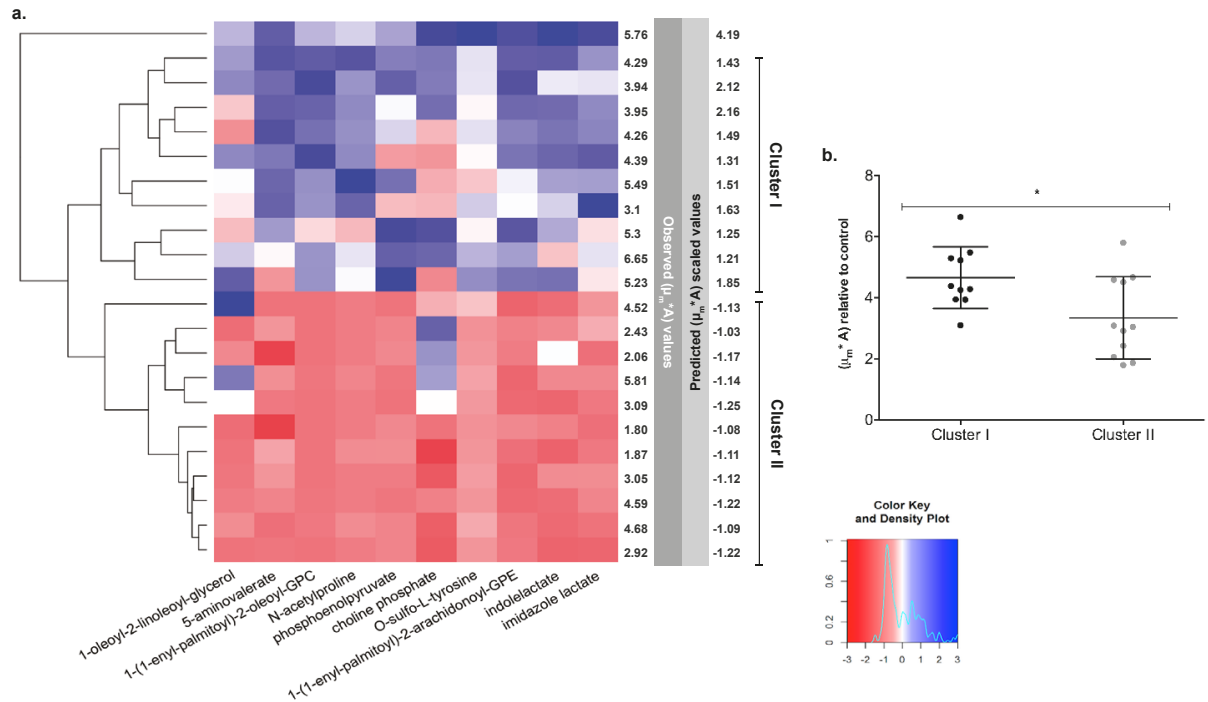

Figure 3. Predictive capacity of the salivary metabolite signature. (a) Hierarchical clustering heatmap displaying relative metabolite concentrations of the signature and re-epithelialization performance for the validation dataset. (b) Re-epithelialization performance observed in clusters I and II. Significant difference was assessed by a two-tailed t-test ( $\left.{ }^{*}, \mathrm{P}<0.05\right)$.

\section{Re-epithelialization capacity of saliva is relatively stable among individuals}

Based on the predicted re-epithelialization capacity of the unstimulated saliva collected during the development of experimental gingivitis, we set out to investigate intra-individual variation during the challenge. To assess the variability of the predicted stimulation of re-epithelialization kinetics for each saliva sample obtained from each individual at the different timepoints during the challenge period (Day 0, Day 2, Day 5, Day 9 and Day 14), we calculated the coefficient of variation $^{183}$. This value describes the variability of the predicted kinetics relative to the mean re-epithelialization capacity of the samples from each of the volunteers in the study (Fig 4a). Notably, the saliva of the individuals that were predicted to have either high (left-end of the graph) or low (right-end of the graph) average re-epithelialization capacity, exhibited low coefficients of variation, indicating that their capacity to promote re-epithelialization was relatively stable and independent of the challenge induced-inflammation (Fig $\mathbf{4 b}$ ). Inherent to the calculation of the coefficient of variation, individuals with average predicted re-epithelialization values close to zero could artificially be concluded to have high variability. Nevertheless, some of the samples of these individuals (e.g. volunteer 5, Fig $4 \mathbf{b}$ ) were predicted to elicit a valid (>2-fold) higher and lower re-epithelialization capacity as compared to the mean, suggesting that the saliva samples of these individuals are legitimately hypervariable in terms of their capacity to stimulate re-epithelialization kinetics. 


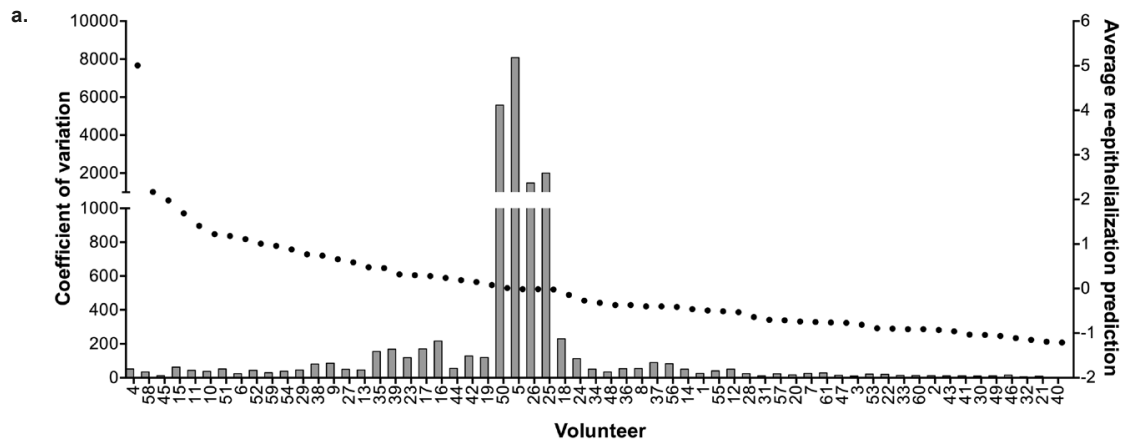

.

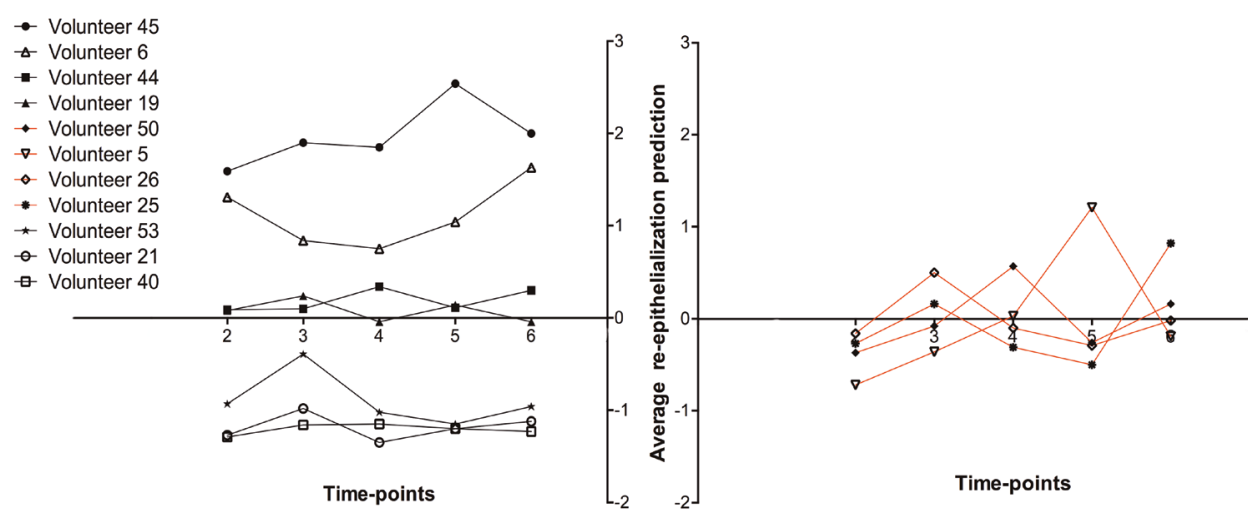

Figure 4. Stability of saliva-induced re-epithelialization among individuals during the experimental gingivitis challenge. (a) The filled circles represent the average re-epithelialization prediction for each volunteer. The bars represent the coefficient of variation obtained per volunteer during the experimental gingivitis challenge. (b) Stability of saliva-induced re-epithelialization for representative volunteers. Symbols with red lines represent individuals with high coefficient of variation.

\section{Relevance of the metabolite signature in relation to gingival bleeding and the magnitude of the response to the challenge}

Periodontal diseases are multifactorial inflammatory diseases that lead to damage of the periodontal tissues supporting the teeth ${ }^{6}$. The gingival bleeding index ${ }^{184}$ has long been used as a reliable clinical parameter for evaluating the inflammatory conditions of the periodontium and over time has been modified in several ways, e.g. scoring for the absence or presence of gingival bleeding on marginal probing (BOMP) ${ }^{177}$. Notably, when we evaluated the association of the re-epithelialization capacity predicted by the metabolite signature with this clinical parameter (percentage of gingival bleeding) at the baseline of the study (Day 0 ) and at the peak of the challenge (Day 14), we discovered that volunteers whose samples were predicted to have a higher stimulatory effect on re-epithelialization had significantly more gingival bleeding $(P=0.005)$ than the group predicted to have samples with lower stimulatory capacity (Fig 5a), indicating that the individuals in the first group would be considered to have increased inflammation of the periodontal tissues. This was further confirmed by a highly significant positive association between the predicted reepithelialization capacity and the measured percentage of gingival bleeding at the baseline and peak of the challenge, 
respectively (Spearman correlation: $r=0.32, P=0.0004$ ). However, the baseline discrimination of the two groups was not able to predict the change in gingival bleeding between the baseline measurement and the measurement at the peak of the challenge ( $\triangle \mathrm{BOMP \%}$; Fig. S5), suggesting that the metabolite signature has no predictive capacity with respect to the magnitude of the response to the challenge. Remarkably, the coefficient of variation of the predicted re-epithelialization stimulation capacity in serial samples of an specific individual (see above) appeared to positively correlate (Spearman correlation: $r=0.33, P=0.009$ ) with the change in bleeding during the trial $(\triangle B O M P \%)($ Fig. 5b), suggesting that the individuals with higher variability of their salivary stimulation of reepithelialization kinetics display relatively higher responsiveness in their periodontium during the experimental gingivitis challenge and could potentially have higher risk to develop gingivitis.

a.

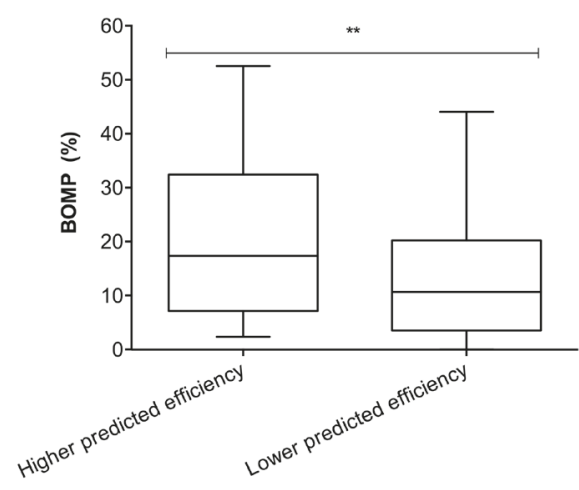

b.

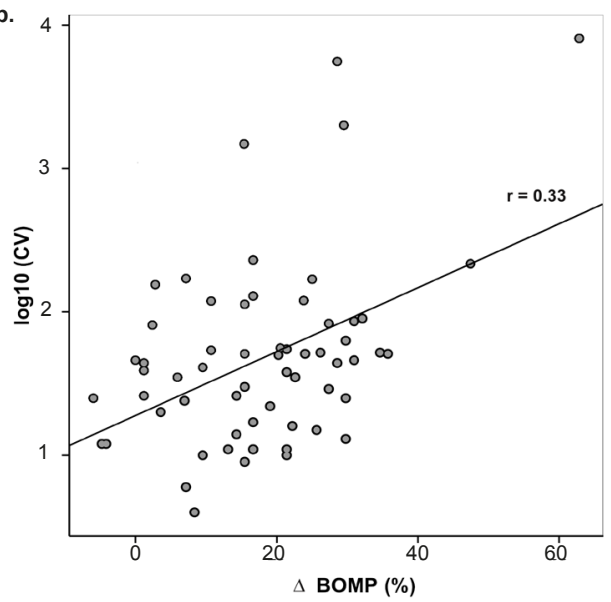

Figure 5. Saliva re-epithelialization capacity in relation to gingival bleeding. (a) Percentage of bleeding upon marginal probing (BOMP\%) for the saliva samples predicted to have higher or lower re-epithelialization capacity at the baseline and at the peak of the challenge period. Significant differences were assessed by two-tailed $t$-tests $\left({ }^{* *}, P<0.01\right)$. (b) Spearman correlation analysis between the coefficient of variation (CV) and the change in percentage of gingival bleeding between the baseline and the peak of the challenge $(P=0.009)$.

\section{Discussion}

Human saliva is secreted from three pairs of major salivary glands (parotid, submandibular, and sublingual glands) as well as numerous minor salivary glands lying beneath the oral mucosa ${ }^{30}$. The protective role of saliva in the maintenance of oral health can be attributed to the presence of its inorganic and organic constituents. Among the organic constituents, saliva contains a myriad of proteins and peptides that have been shown to modulate different aspects of oral health, including enzymatic digestion, food tasting and swallowing, lubrication, protection against pathogenic microorganisms, remineralization, and stimulation of tissue repair ${ }^{25,28-30,185}$.

In recent years, metabolomic analysis of human saliva have revealed that this biofluid is also a rich source of low molecular weight metabolites such as lipids, amino acids, vitamins, organic acids, thiols, carbohydrates, and their derivatives, which can be important indicators of physiological or pathological states ${ }^{23,186,187}$. Different studies have shown that specific 
changes in the metabolic profiles of saliva can be associated with the presence of periodontal diseases ${ }^{188}$ and oral cancer ${ }^{189}$. Importantly, metabolites from blood can pass into the saliva through transcellular, intracellular or paracellular routes involving passive diffusion or active transport within the salivary glands or the gingival sulcus ${ }^{22}$. As a consequence salivary metabolites have the potential to provide a window to the rest of the body that has been used in the early detection of bacterial infections ${ }^{190}$, breast cancer, and pancreatic cancer ${ }^{189}$.

In this study we used the metabolite profiles of 305 unstimulated saliva samples collected from 61 healthy volunteers at 5 timepoints during an experimental gingivitis challenge intervention ${ }^{170}$ to investigate their influence on reepithelialization kinetics using a quantitative in vitro scratch model ${ }^{155}$. The re-epithelialization kinetics (in particular the overall performance value $\left.\mu_{m}{ }^{*} A\right)$ obtained in a small training set of saliva samples $(n=63)$ enabled the identification of a specific salivary metabolite signature (10 metabolites) that allowed the prediction of the re-epithelialization stimulatory capacity of saliva samples obtained in an experimental gingivitis challenge model. Importantly, regression analyses revealed that the metabolite signature was correlated much stronger with the observed re-epithelialization kinetics than single metabolites within the signature, which clearly illustrates the added value of combinatorial multi-factorial biomarkers as compared to single factor biomarkers.

The metabolite signature (Table 1) contained several metabolites that are associated with host-microbe interactions, cellular degradation, and in some cases inflammation. The 1-(1-enyl-palmitoyl)-2-arachidonyl-GPE and 1-(1-enylpalmitoyl)-2-oleoyl-GP( are plasmalogens, a type of membrane phospholipids that contain a vinyl-ether bond at the sn-1 position of the glycerophospholipid backbone ${ }^{191}$. Plasmalogens comprise $~ 18 \%$ of the phospholipid mass in humans and help maintaining the physical bilayer properties of cell membranes ${ }^{192}$, while the hydrogen atoms adjacent to the vinylether bond are preferentially oxidized when exposed to free radicals and thereby can act as antioxidants ${ }^{193,194}$. Untargeted metabolic profiling of saliva from healthy and periodontal patients, revealed an association between periodontal disease and elevated levels of degradation products of several macromolecules, including glycerolphospholipids ${ }^{188}$. Moreover, elevated concentrations of various plasmalogen derivates in serum and/or synovial fluid have been correlated with the severity of inflammatory diseases like obesity-associated osteoarthritis $(\mathrm{OA})$ and rheumatoid arthritis in both human ${ }^{195}$ and mice ${ }^{196}$. Interestingly, the metabolite 1-oleoyl-2-linoeoyl-glycerol (diacylglycerol; DAG) has recently been associated with cell damage and the formation of spatially organized lipid domains around the site of injury ${ }^{197}$, in which DAG was shown to be required for regulation of GTPases, Rho and Cdc42 involved in cortical cytoskeleton repair ${ }^{197}$. Choline phosphate (ChoP) is an intermediate in the synthesis of phosphatidylcholine, a major phospholipid of eukaryotic cell membrane-es ${ }^{198}$. ChoP has been shown to potentiate the mitogenic effect of insulin in NIH $3 \mathrm{T3}$ fibroblasts ${ }^{199,200}$, and therefore it remains unclear why increasing concentrations of this metabolite in saliva resulted in reduced re-epithelialization capacity. Taken together, higher concentrations of these lipid metabolites in saliva may result from increased mucosal damage and cellular decay through enhanced host-microbe interactions, as was already proposed by the authors of the salivary metabolome study ${ }^{188}$. Moreover, such elevated host-microbe interplay is in agreement with the higher levels of modified amino acids that (potentially) result from bacterial metabolism, i.e. 5 -aminovalerate, imidazole lactate, indolelactate, $\mathrm{N}$-acetylproline, and 0-sulfo-L-tyrosine. In particular, elevated levels of 5-aminovalerate, a metabolite of the foul-smelling diamine cadaverine that is produced by microbial protein hydrolysis ${ }^{201}$, has been associated with patients with chronic periodontitis ${ }^{202}$. Finally, many secreted and transmembrane proteins that play a role in various biological processes involved in wound repair (e.g. blood coagulation, optimal rolling of leukocytes on endothelial cells upon tissue damage, chemokine receptor signalling, and extracellular matrix remodelling) are commonly post-translationally modified when they pass through the trans- 
golgi compartment to introduce 0 -sulfo-tyrosine residues ${ }^{203-205}$. The elevated levels of this metabolite in saliva may reflect increased bacterial hydrolysis of host-derived proteins.

Overall, the metabolite signature identified in this study encompasses metabolites that could reflect enhanced hostmicrobe interactions at the mucosal tissues in the oral cavity, and may thus reflect a chemical signature for mild mucosal damage. Higher concentrations of most of the metabolites in the signature (with the exception of choline phosphate) was associated with accelerated gingival wound repair in vitro, which could reflect the cellular response to the proposed mucosal damage, and which is in agreement with the signature's association with elevated gingival bleeding scores. However, in many individuals the metabolite signature appeared quite stable over time, and this low-level intraindividual variation includes the samples taken during the experimental gingivitis challenge period. This observation implies that although the interplay between the oral mucosa and the microbiota is anticipated to amplify during the challenge period and (generally) induce an increase in gingival bleeding, this challenge did not have a strong impact on the metabolites in the signature. Intriguingly, despite the common low level of intra-personal variation, a subset of the participating individuals displayed a considerable variability of the signature at different timepoints of sampling, which was significantly associated with a higher increase in gingival bleeding scores (responsiveness) during the gingivitis challenge. One may speculate that stability of host-microbe interactions in the oral cavity, and its corresponding salivary metabolite signature, could reflect a healthy state. In such context, individuals with unstable host-microbe interactions and higher variability of the metabolite signature may be at higher risk to respond more strongly under a challenge, like the gingivitis challenge model tested in this study.

The mechanism by which the combination of these metabolites is able to modulate oral re-epithelialization remains unclear at this stage. They may directly or indirectly activate wound-repair responses in gingival cells, which is measured as enhanced re-epithelialization in the in vitro scratch assay, and this model system may serve as an appropriate system to unravel this mechanism of action of the metabolite signature. Therefore, the identified signature may open promising avenues for the development of novel treatment strategies in patients with mucositis, recurrent oral aphthous ulcers or Sjögren's syndrome, disease states that are all characterized by impaired wound healing ${ }^{77,206}$.

\section{Acknowledgements}

We thank the clinical team at the Academic Centre for Dentistry Amsterdam (ACTA) and the University of Amsterdam and Vrije Universiteit Amsterdam for planning, reviewing and executing the experimental gingivitis challenge intervention. 


\section{Supplementary data}

a.

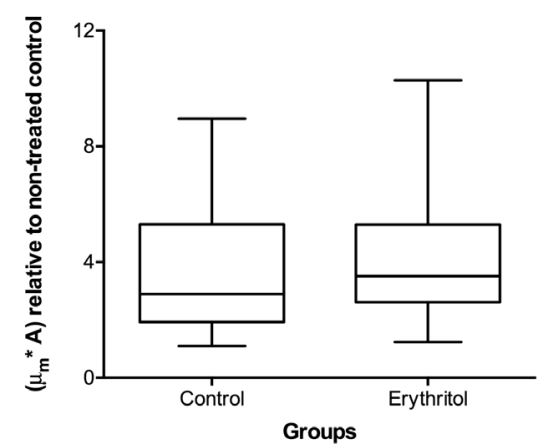

b.

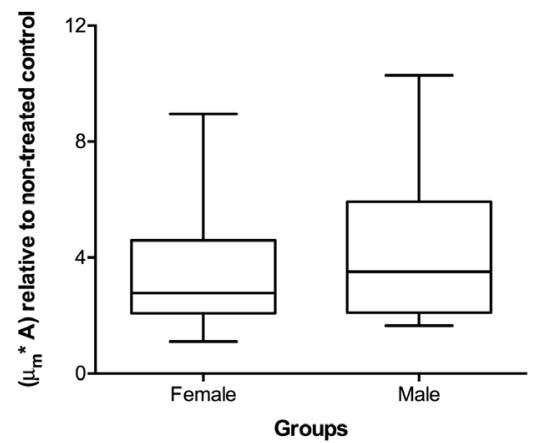

Figure S1. Effect of erythritol and gender on re-epithelialization kinetics. (a) Salivary re-epithelialization capacity was not influenced by daily intake of erythritol ( $P=0.44$ ). Control group, $n=72$, erythritol group, $n=31$. (b) Gender did not influence the capacity of unstimulated saliva to promote in vitro re-epithelialization $(P=0.12)$. Significant differences were assessed by a two-tailed t-test.

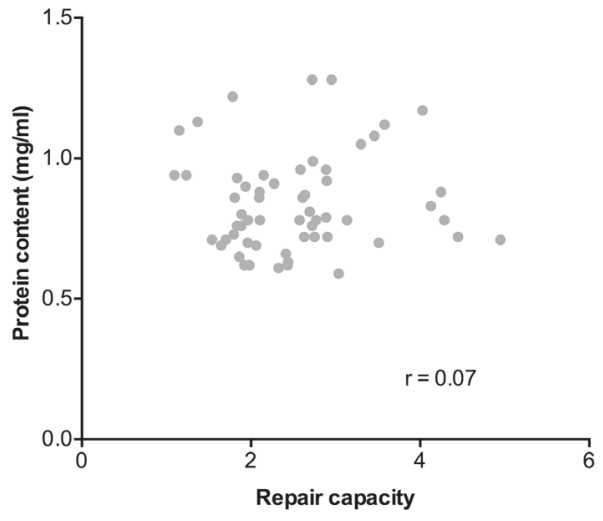

Figure S2. Correlation between unstimulated saliva total protein content and its re-epithelialization capacity. Association between total protein content $(\mathrm{mg} / \mathrm{ml})$ and the capacity of unstimulated saliva to promote wound repair on gingival epithelial cells assessed by a Spearman correlation $(n=58, P=0.58)$. 
a.

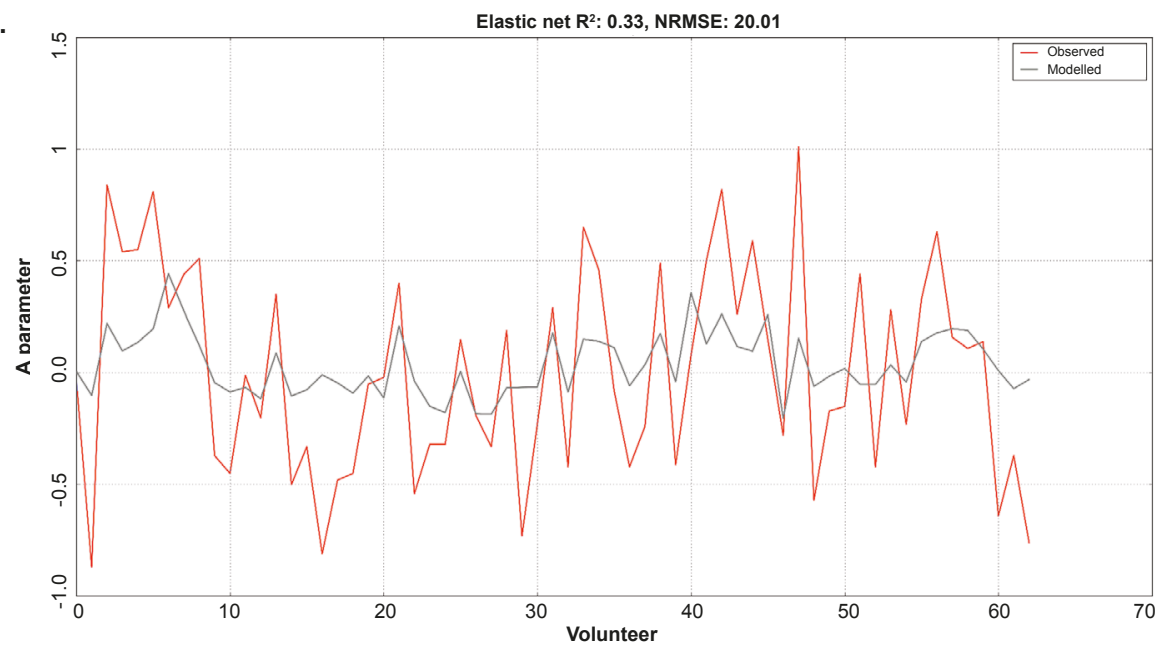

b.

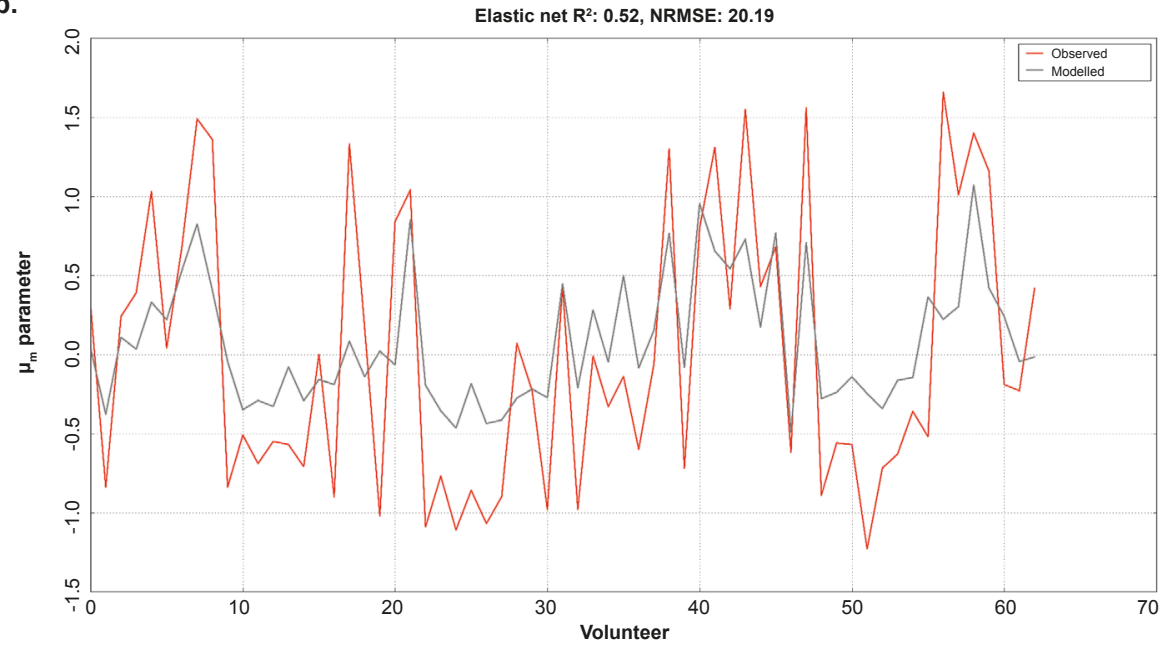

Figure S3. Elastic net regression. (a) Performance of the elastic net regression through the A parameter values. (b) Performance of the elastic net regression through the $\mu_{\mathrm{m}}$ parameter values. 

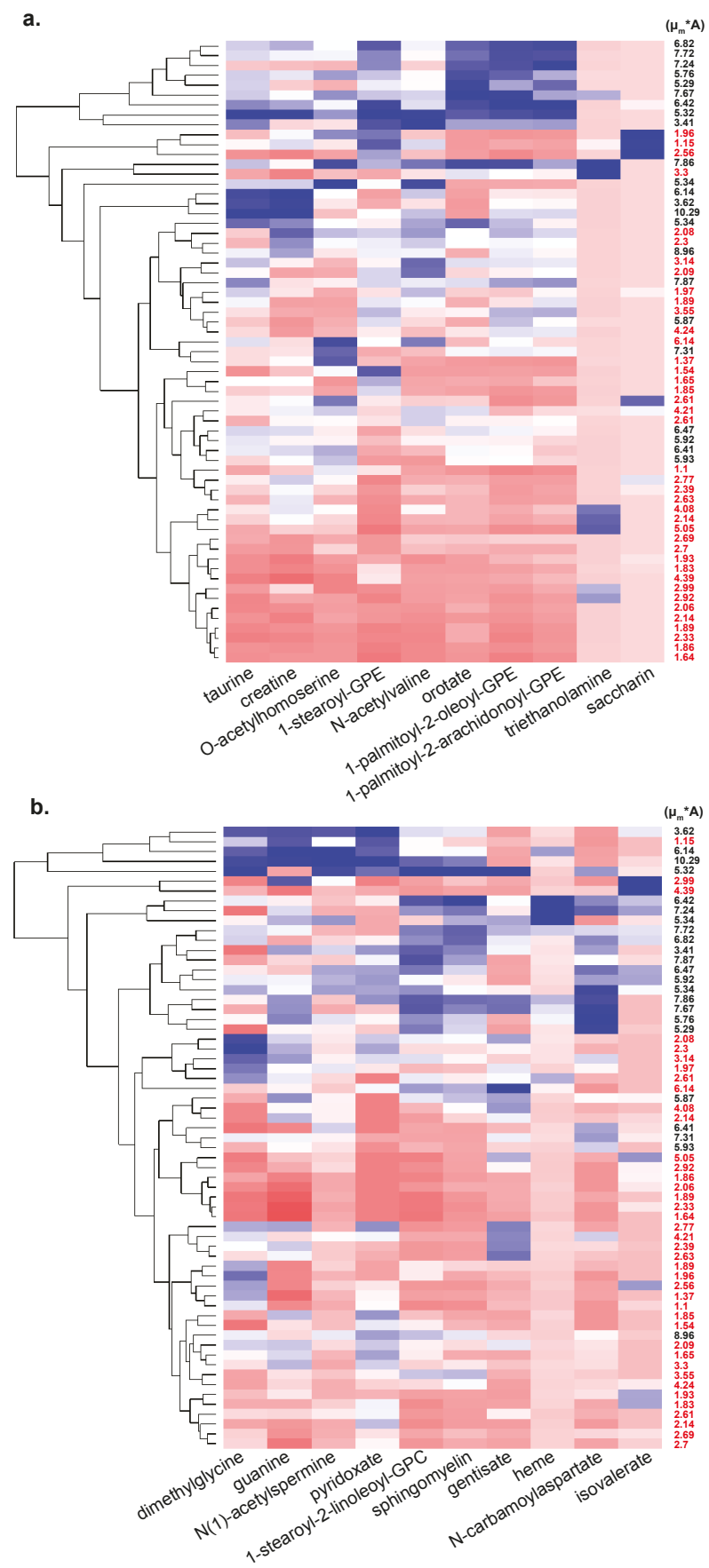

Figure S4. Heatmaps with hierarchical clustering. (a) and (b) are exemplary and representative heatmaps of randomly selected metabolites in the dataset. Re-epithelialization performance values are colour-coded according to their positioning in cluster I (black) or II (red) derived from the hierarchical clustering of the metabolite signature. 


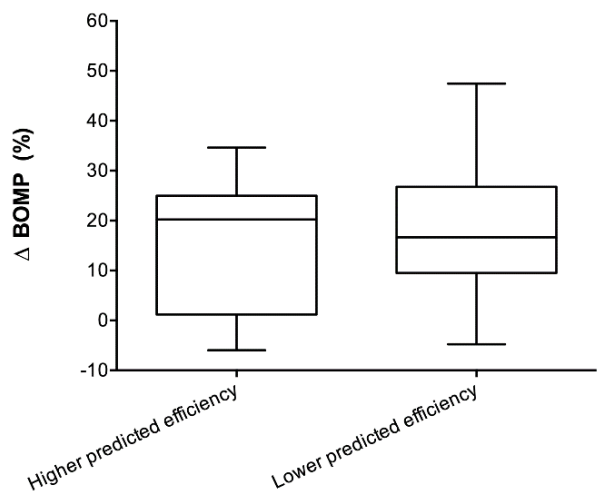

Figure S5. Predictive capacity of the metabolite signature in relation to the response to the challenge. Response to the challenge reflected by the change in the percentage of gingival bleeding in the groups predicted to have saliva samples with higher and lower re-epithelialization capacity at the baseline of the study.

Table S1. Re-epithelialization kinetics obtained for the training dataset. Re-epithelialization kinetic parameters obtained for 63 randomly selected samples collected during the challenge intervention phase (Day 0 to Day 14, time-point 2 to 6 ).

\begin{tabular}{|c|c|c|c|c|c|c|}
\hline Volunteer & Time-point & Mean, $\mu_{\mathrm{m}}$ & $S D, \mu_{m}$ & Mean, A & SD, A & $\mu_{m}{ }^{*} A$ \\
\hline 1 & 2 & 1.861 & 0.075 & 1.401 & 0.048 & 2.607 \\
\hline 1 & 3 & 1.849 & 0.002 & 1.422 & 0.032 & 2.629 \\
\hline 1 & 4 & 2.727 & 0.475 & 1.542 & 0.163 & 4.206 \\
\hline 1 & 5 & 2.063 & 0.745 & 1.341 & 0.162 & 2.767 \\
\hline 1 & 6 & 1.185 & 0.121 & 2.015 & 0.201 & 2.388 \\
\hline 3 & 2 & 1.439 & 0.683 & 1.34 & 0.05 & 1.928 \\
\hline 3 & 3 & 1.908 & 0.264 & 1.124 & 0.161 & 2.144 \\
\hline 3 & 4 & 2.494 & 0.502 & 1.76 & 0.116 & 4.39 \\
\hline 3 & 5 & 1.865 & 0.084 & 1.375 & 0.118 & 2.564 \\
\hline 3 & 6 & 1.707 & 0.016 & 1.073 & 0.124 & 1.831 \\
\hline 5 & 3 & 4.077 & 0.669 & 2.198 & 0.248 & 8.959 \\
\hline 8 & 2 & 1.403 & 0.68 & 1.523 & 0.202 & 2.137 \\
\hline 8 & 3 & 3.262 & 0.356 & 1.547 & 0.276 & 5.047 \\
\hline 8 & 4 & 3.75 & 1.336 & 1.087 & 0.158 & 4.076 \\
\hline 8 & 5 & 2.593 & 0.247 & 1.124 & 0.047 & 2.915 \\
\hline 8 & 6 & 2.416 & 0.298 & 1.239 & 0.144 & 2.994 \\
\hline 9 & 3 & 2.407 & 0.467 & 2.22 & 0.473 & 5.343 \\
\hline 9 & 4 & 2.846 & 0.12 & 2.157 & 0.327 & 6.138 \\
\hline 10 & 3 & 3.104 & 0.812 & 1.719 & 0.174 & 5.337 \\
\hline 10 & 4 & 2.714 & 0.76 & 2.385 & 0.238 & 6.474 \\
\hline 10 & 5 & 3.434 & 0.684 & 1.725 & 0.08 & 5.925 \\
\hline 11 & 2 & 3.913 & 0.593 & 2.007 & 0.066 & 7.855 \\
\hline 11 & 3 & 3.717 & 0.232 & 2.064 & 0.129 & 7.675 \\
\hline 11 & 4 & 3.102 & 0.701 & 1.856 & 0.093 & 5.758 \\
\hline 15 & 6 & 3.218 & 0.896 & 1.653 & 0.078 & 5.319 \\
\hline 16 & 2 & 1.703 & 0.069 & 1.159 & 0.172 & 1.975 \\
\hline 16 & 3 & 1.58 & 0.226 & 1.197 & 0.021 & 1.891 \\
\hline 16 & 4 & 2.359 & 0.004 & 1.329 & 0.074 & 3.135 \\
\hline 16 & 5 & 1.822 & 0.227 & 1.148 & 0.054 & 2.092 \\
\hline 16 & 6 & 2.282 & 0.223 & 1.494 & 0.233 & 3.408 \\
\hline 17 & 3 & 2.461 & 0.537 & 2.385 & 0.414 & 5.869 \\
\hline 21 & 2 & 1.314 & 0.365 & 1.248 & 0.177 & 1.639 \\
\hline 21 & 3 & 1.649 & 0.038 & 1.249 & 0.015 & 2.06 \\
\hline 21 & 4 & 1.803 & 0.147 & 1.293 & 0.023 & 2.331 \\
\hline 21 & 5 & 1.521 & 0.029 & 1.24 & 0.065 & 1.886 \\
\hline 21 & 6 & 1.349 & 0.081 & 1.38 & 0.016 & 1.862 \\
\hline 26 & 3 & 2.226 & 0.097 & 0.934 & 0.167 & 2.08 \\
\hline 26 & 4 & 2.84 & 1.044 & 0.811 & 0.147 & 2.302 \\
\hline 26 & 5 & 2.188 & 0.404 & 1.195 & 0.077 & 2.615 \\
\hline 35 & 3 & 2.848 & 0.606 & 1.857 & 0.299 & 5.289 \\
\hline 35 & 4 & 3.785 & 0.688 & 2.079 & 0.25 & 7.868 \\
\hline
\end{tabular}




\begin{tabular}{|c|c|c|c|c|c|c|}
\hline Volunteer & Time-point & Mean, $\mu_{m}$ & $S D, \mu_{m}$ & Mean, A & SD, A & $\mu_{m}{ }^{*} A$ \\
\hline 36 & 2 & 1.734 & 0.209 & 1.557 & 0.149 & 2.699 \\
\hline 36 & 3 & 1.564 & 0.04 & 1.718 & 0.189 & 2.687 \\
\hline 36 & 4 & 1.848 & 0.217 & 1.922 & 0.133 & 3.552 \\
\hline 36 & 5 & 1.785 & 0.007 & 1.849 & 0.047 & 3.301 \\
\hline 36 & 6 & 2.09 & 0.071 & 2.029 & 0.097 & 4.242 \\
\hline 45 & 2 & 3.727 & 0.437 & 2.07 & 0.301 & 7.717 \\
\hline 45 & 3 & 3.966 & 0.216 & 1.825 & 0.453 & 7.239 \\
\hline 45 & 5 & 3.82 & 1.001 & 1.68 & 0.045 & 6.417 \\
\hline 45 & 6 & 3.463 & 0.733 & 1.97 & 0.131 & 6.822 \\
\hline 51 & 3 & 1.905 & 0.371 & 1.903 & 0.692 & 3.625 \\
\hline 51 & 4 & 3.983 & 0.07 & 2.583 & 0.298 & 10.289 \\
\hline 51 & 6 & 3.585 & 0.089 & 1.714 & 0.342 & 6.144 \\
\hline 54 & 4 & 2.806 & 0.247 & 2.112 & 0.164 & 5.926 \\
\hline 54 & 5 & 3.453 & 0.221 & 2.118 & 0.19 & 7.314 \\
\hline 54 & 6 & 2.662 & 0.385 & 2.407 & 0.189 & 6.408 \\
\hline 55 & 2 & 1.516 & 0.073 & 0.761 & 0.009 & 1.153 \\
\hline 55 & 3 & 1.576 & 0.168 & 0.701 & 0.046 & 1.104 \\
\hline 55 & 4 & 1.329 & 0.09 & 1.032 & 0.025 & 1.371 \\
\hline 55 & 5 & 1.699 & 0.174 & 1.151 & 0.116 & 1.956 \\
\hline 55 & 6 & 1.531 & 0.052 & 1.003 & 0.056 & 1.535 \\
\hline 57 & 3 & 2.192 & 0.625 & 0.842 & 0.218 & 1.845 \\
\hline 57 & 6 & 1.437 & 0.021 & 1.15 & 0.206 & 1.652 \\
\hline
\end{tabular}

Table S2. Feature selection. Elastic net regression with stability selection was performed to select a set of metabolites that were associated to reepithelialization kinetics using the $\mu_{\mathrm{m}}$ and $\mathrm{A}$ parameters.

\begin{tabular}{|c|c|c|c|c|}
\hline \multirow{6}{*}{ 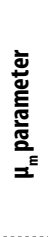 } & Feature Selected & Description & Stability & Average Weight \\
\hline & 1-(1-enyl-palmitoyl)-2-arachidonoyl-GPE (P-16:0/20:4)* & Plasmalogen & 0.60 & 0.04 \\
\hline & 1-(1-enyl-palmitoyl)-2-oleoyl-GPC (P-16:0/18:1)* & Plasmalogen & 0.73 & 0.08 \\
\hline & nicotinate & Nicotinate and Nicotinamide Metabolism & 0.67 & 0.04 \\
\hline & 0-sulfo-L-tyrosine & Chemical & 1.00 & 0.15 \\
\hline & urea & Urea cycle; Arginine and Proline Metabolism & 0.68 & -0.03 \\
\hline \multirow{6}{*}{ 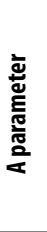 } & 1-stearoyl-GPS $(18: 0)^{*}$ & Lysolipid & 0.72 & -0.03 \\
\hline & 2-piperidinone & Food Component/Plant & 0.71 & 0.03 \\
\hline & 4-hydroxyphenylacetate & Phenylalanine and Tyrosine Metabolism & 0.73 & 0.03 \\
\hline & glycosyl-N-palmitoyl-sphingosine & Sphingolipid Metabolism & 0.76 & 0.04 \\
\hline & imidazole lactate & Histidine metabolism & 1.00 & 0.07 \\
\hline & phenol sulfate & Phenylalanine and Tyrosine Metabolism & 0.81 & -0.04 \\
\hline
\end{tabular}





\title{
Chapter
}

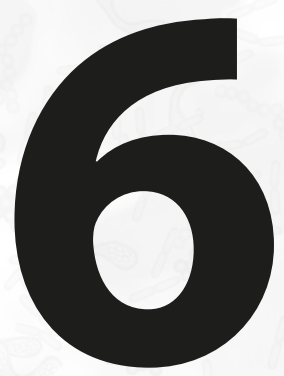

\section{Identification of Oral Commensal Bacteria Related to Gingival Bleeding and Re-Epithelialization with Disease-Risk Signature Potential}

\author{
Marcela M. Fernandez-Gutierrez ${ }^{1,2}$, \\ Sultan Imangaliyev ${ }^{1,3,4}$, \\ Bernd W. Brandt ${ }^{1,4}$, \\ Jos Boekhorst ${ }^{5}$, \\ Bart Keijser ${ }^{1,3,4}$, \\ Bruno G. Loos ${ }^{1,6}$, \\ Michiel Kleerebezem ${ }^{1,2^{*}}$
}

\footnotetext{
' TI Food and Nutrition, Nieuwe Kanaal 9-A, 6709 PA, Wageningen, The Netherlands.

${ }^{2}$ Host-Microbe Interactomics Group, Department of Animal Sciences, Wageningen University \& Research, De Elst 1, 6708 WD, Wageningen, The Netherlands.

${ }^{3}$ TNO Microbiology and Systems Biology, Utrechtseweg 48, $3704 \mathrm{HE}$, Zeist, The Netherlands.

${ }^{4}$ Department of Oral Biochemistry, Academic Centre for Dentistry Amsterdam (ACTA), University of Amsterdam and Vrije

Universiteit Amsterdam, Gustav Mahlerlaan 3004, 1081 LA, Amsterdam, The Netherlands.

${ }^{5} \mathrm{NIZO}$ Food Research BV, Kernhemseweg 2, 6718 ZB, Ede, The Netherlands.

${ }^{6}$ Department of Periodontology Academic Centre for Dentistry Amsterdam (ACTA), University of Amsterdam and Vrije

Universiteit Amsterdam, Gustav Mahlerlaan 3004, 1081 LA, Amsterdam, The Netherlands.
} 
The oral mucosa constitutes a physical barrier that protects the underlying tissue from bacterial infection and also plays an active role in innate immunity. However, in presence of active periodontal disease the epithelial integrity is breached, resulting in bacterial invasion, inflammation, and subsequent destruction of the connective tissue supporting the teeth. We identified operational taxonomical units (OTUs) in a cross-sectional study that were positively or negatively associated with gingival bleeding scores, which reflect the inflammatory status of the periodontium. Representative species of the identified OTUs were screened for their effect on oral re-epithelialization using an in vitro model. This revealed that the group of bacteria negatively associated with gingival bleeding scores elicited significantly increased re-epithelialization kinetics as compared to the positively associated group. In addition, the relative abundance of the selected species was investigated during the development of mild gingivitis in a two-week experimental gingivitis challenge. The results strongly corroborated the association of the identified species with the gingival bleeding intensity in this challenge-model cohort. Moreover, redundancy and correlation analyses showed that changes in the relative abundance during the challenge-model of several microbes, including Selenomonas sputigena and Veillonella dispar, was linked to the individual's response to the experimental gingivitis challenge. In addition, microbiota composition signatures at the start of the challenge were shown to be useful to predict disease risk, illustrating the potential of these composite 'biomarker' signatures in disease diagnostics. 


\section{Introduction}

The human oral cavity is a complex and dynamic ecosystem that is heavily colonized by a variety of microbial communities that differ depending on the niche within the oral cavity, which include saliva, hard dental surfaces, mucosal sites, and anaerobic pockets ${ }^{34}$. Thus, oral bacteria have adapted strategies that facilitate their colonization and growth in particular niches of the oral cavity, while the host normally limits their growth by a combination of innate and adaptive immune responses ${ }^{207}$. This dynamic equilibrium between the oral microbiome and the host immune system plays a crucial role in oral health and disease ${ }^{48}$. Commensal microorganisms usually coexist peacefully with the host. They support maintenance of a functional immune system, prevent pathogenic microorganisms from attaching to and colonizing healthy tissues, and regulate epithelial development ${ }^{208}$. When the balance between the microbiome and the host is disturbed, a subset of species can escape from the host restraint mechanisms and initiate disease. Periodontal diseases are the most common chronic inflammatory disorders initiated by bacteria in humans ${ }^{6,102}$. The inflammatory responses caused by periodontal diseases can be grouped into two clinical classifications: those only affecting the gingiva (i.e. gingivitis), and the ones deeply progressing into the tissues of the periodontium, including the periodontal ligament and the alveolar bone (i.e. periodontitis). Gingivitis is an inflammatory response that results from increased accumulation of bacteria on the teeth, but it can usually be prevented or reversed with appropriate oral hygien ${ }^{209}$. In contrast, periodontitis is characterized by severe inflammation of the periodontal ligament, which leads to loss of collagen attachment between the tooth and the bone, irreversible reabsorption of the alveolar bone, and eventually tooth loss ${ }^{210}$. Furthermore, certain periodontal pathogens such as Porphyromonas gingivalis and Fusobacterium nucleatum have been shown to efficiently supress wound repair in vitro, which may contribute to the initiation or progression of the disease ${ }^{79,80}$.

We previously conducted a cross-sectional observational study ${ }^{211}$ in which we determined the microbiota composition of seven different oral cavity niches of 268 systemically healthy young adults and performed clinical examinations to determine the inflammatory state of their periodontal tissues based on the gingival bleeding scores ${ }^{184}$. In the present study, we aimed to identify microorganisms by elastic net regression that were associated with higher or lower gingival bleeding in the cross-sectional study. The influence of the identified species on gingival re-epithelization was then assessed using a high-throughput scratch assay as a way to identify a potential mechanism underlying their association. We could establish that the group of microorganisms negatively associated with gingival bleeding significantly enhanced in vitro re-epithelialization as compared to the positively associated group. Furthermore, when we evaluated the relative abundance of these bacteria in unstimulated saliva obtained from individuals that underwent a two-week experimental gingivitis challenge $(n=41)$, we were able to confirm the association of most of these species with gingival bleeding. In particular, higher relative abundances of Actinomyces oris/viscosus/naes/undii, Rothia dentocariosa and Veillonella dispar had the strongest negative correlations with gingival bleeding. Furthermore, a higher relative abundance of either Porphyromonas catoniae, Selenomonas sputigena, Leptrotrichia buccalis and Streptococcus anginosus was associated with increased gingival bleeding. Moreover, changes in relative abundances of particular microbes, such as Veillonella dispar and Selenomonas sputigena, could be significantly linked to the individual's response to the experimental gingivitis challenge. Finally, oral microbiota composition signatures at the start of the challenge period were shown to have diagnostic potential to predict disease risk. 


\section{Materials and Methods}

\section{Clinical studies design and ethical considerations}

The cross-sectional observational study consisted of 268 systemically healthy young adults between 18 and 32 years of age without apparent caries or periodontitis. The inclusion and exclusion criteria were previously described by Prodan et. al. ${ }^{176}$ and Oliveira et. al. ${ }^{177}$. The experimental gingivitis challenge consisted of two weeks in which 41 systemically healthy volunteers (i.e. control group) between 18 and 55 years of age were restrained from any form of oral hygiene ${ }^{170,212}$. The inclusion and exclusion criteria were previously described by Prodan et. al. ${ }^{170}$. The cross-sectional study protocol was reviewed by the Medical Ethics Committee of Amsterdam (2012_210\#B2012406) and registered at the Dutch Trial Register (NTR3649). The experimental gingivitis challenge protocol was reviewed and approved by the Medical Ethics Committee of the Vrije Universiteit Medical Centre (2014_505) and registered at the Central Committee on Research Involving Human Subjects (CCMO). Both studies were carried out within the framework of the Top Institute Food and Nutrition (TIFN) at the Academic Centre for Dentistry Amsterdam (ACTA) and conducted according to the Declaration of Helsinki ${ }^{175}$ of the World Medical Association. All volunteers signed an informed consent form.

\section{Clinical examination}

The subjects in the cross-sectional study were instructed not to eat or drink (except for water) after 12:00 o'clock of the night before the appointment as well as to refrain from any form of oral hygiene procedure 24 hours before the appointment. Assessment of bleeding on marginal probing (BOMP) and upon pocket probing (BOPP) were described by Oliveira et. al. ${ }^{177}$. To assess BOMP, the teeth (excluding third molars) were clinically examined at six sites per tooth (disto-vestibular, vestibular, mesio-vestibular, disto-lingual, lingual and mesio-lingual) in two contralateral quadrants (i.e. one upper and one lower), amounting to 84 observations per individual. The number of gingival units that bled within 30 seconds upon marginal probing was recorded with scores from 0 to 2 ( $0=$ no bleeding, 1 = pinprick, 2 = excessive bleeding). The BOPP was recorded by inserting a force controlled probe parallel to the root surface and towards the perceived location of the root apex. The BOPP was determined in all four quadrants, amounting to 186 observations per individual of which 84 (50\%) were recorded immediately after the BOMP assessment on the same site ${ }^{177}$. The number of pocket units that bled within 30 seconds after probing was recorded $(0=$ no bleeding, 1 = bleeding). The bleeding index for each individual was calculated with the intention to integrate all available data of BOMP and BOPP measurements. To this end, the BOMP scores were binarized (i.e. the ' 2 ' scores were converted to ' $1{ }^{\prime}$ ) and subsequently the BOMP scores of ' 1 ' were summed up with the BOPP scores of ' 1 ' for all sites measured, and normalized by dividing the summed value by the total number of observations (i.e. 252). Volunteers enrolled in the experimental gingivitis challenge were instructed to follow the same guidelines as in the cross-sectional study for the assessment at the start of the challenge period (Day 0). In this study, BOMP scores were recorded before the start of the challenge (Day 0 ) and at the peak of the challenge (Day 14) period. Gingival bleeding was expressed as a percentage of sites that bled upon probing and the response to the challenge was expressed by calculating the difference between the percentage of gingival bleeding at the peak (Day 14 ) and at the beginning (Day 0 ) of the challenge ( $\triangle \mathrm{BOMP} \%$ ). 


\section{S rRNA gene amplicon sequencing}

Microbial DNA isolation, sequencing of the V4 hypervariable region of the 16S rRNA gene on the Illumina MiSeq platform (Illumina, Eindhoven, $\mathrm{NL}$ ), and data processing were previously described by Zaura et. al. ${ }^{211}$. The microbiota composition was determined for seven different niches: interproximal, subgingival lower jaw, subgingival upper jaw, supragingival, tongue posterior, tongue anterior, and unstimulated saliva. Furthest neighbour hierarchical clustering was used to cluster the sequences into operational taxonomic units (OTUs) using a 97\% similarity threshold. Consensus taxonomy for the OTUs was generated using a confidence threshold of $60 \%$. The Human Oral Microbiome Database (HOMD) ${ }^{213}$, Greengenes Database (http://greengenes.lbl.gov/) and the NCBI database were used to assign a taxonomic name to the OTUs representative sequences at species-level. The most abundant sequences were compared by BLASTN analysis ${ }^{214}$ using the HOMD (RefSeq version 14.5), Greengenes and NCBI Microbial 165 rRNA databases. Different species with identical sequence identity scores were combined to species 'groups' (e.g. Streptococcus oralis/dentisani/tigurinus/mitis/infantis).

\section{Regularized feature selection analysis}

Elastic net regression with stability selection was used to select OTUs that were related to the gingival bleeding index (see above) of the individuals in the cross-sectional study. Elastic net is a regularized regression method that linearly combines the penalties of the least absolute shrinkage and selection operator (LASSO) and ridge methods ${ }^{178}$. The elastic net with stability selection was implemented in Python using the scikit-learn package v.0.16.1 ${ }^{180}$, and optimized to minimize the root-mean-square error (RMSE). The microbiome data (0TU table) was pre-processed by removing rare 0TUs (i.e. OTUs with less than 5 reads per individual in less than 10 individuals) after which the data was sample-wise normalized to a sum of one. Finally, the microbiome data was zero-mean scaled and then divided by the standard deviation (unit-variance). Features were selected based on their stability after 100 runs (iterations) of feature selection model on random data shuffles using the stability selection procedure ${ }^{179}$. An average weight is assigned to each feature with its corresponding weight standard deviation calculated after completing all iterations. The fractional weight (weight standard deviation/ average weight) was calculated as a measure of the strength and stability of the associations, where values closer to zero had the most stable association with gingival bleeding. A fractional weight of 0.6 served as upper threshold, whereas -0.6 served as lower threshold.

\section{Cell line culture and maintenance}

Gingival epithelial cells (Ca9-22, JCRB0625) were purchased from the National Institute of Biomedical Innovation JCRB Cell Bank (Osaka, Japan). The cells were grown in Dulbecco's Modified Eagle Medium (DMEM) Glutamax (Gibco, Invitrogen, Paisley, UK) supplemented with $10 \%$ fetal calf serum (FCS), $100 \mathrm{U} / \mathrm{mL}$ penicillin, and $100 \mu \mathrm{g} / \mathrm{mL}$ streptomycin (SigmaAldrich, MO, USA). The cell line was incubated at $37^{\circ} \mathrm{C}$ in a humidified atmosphere containing $5 \% \mathrm{CO}_{2}$ and were passaged every three to four days.

\section{Bacterial strains and culturing conditions}

The bacterial strains used in this study are listed in Table 1. Porphyromonas catoniae ATCC 51270 was purchased from the American Type Culture Collection. Porphyromonas gingivalis W83 was kindly provided by Dr. Alexa M. G. A. Laheij from ACTA (Academic Centre for Dentistry Amsterdam, Amsterdam, The Netherlands). Streptococcus mitis HSISM1 
and Veillonella parvula HSIVP1 were a gift from Dr. Tom van den Bogert from the Laboratory of Microbiology of the Wageningen University (Wageningen, The Netherlands). All other strains were purchased from the German Collection of Microorganisms and Cell Cultures (DSMZ). The bacterial strains were grown under the conditions and culture media recommended by DSMZ (https://www.dsmz.de) and when required, anaerobic conditions were obtained with the GasPak $^{\mathrm{TM}}$ EZ Anaerobe Container System Sachets (Becton, Dickinson and Company, NJ, USA). Facultative anaerobes were cultured at $37^{\circ} \mathrm{C}$ in a humidified incubator with $5 \% \mathrm{CO}_{2}$.

Table 1. Bacterial strains used in this study.

\begin{tabular}{|c|c|c|c|}
\hline Species & Culture collection & Strain/Isolate & Origin \\
\hline Actinomyces oris & DSM 23056 & W1053, WVU 474 & Human sputum \\
\hline Actinomyces viscosus & DSM 43798 & Ny 334 & Human mouth \\
\hline Fusobacterium nucleatum subsp. animalis & DSM 19679 & & Animal colon \\
\hline Fusobacterium nucleatum subsp. nucleatum & DSM 15643 & $1612 A$, VPI 4355 & Cervicofacial lesion \\
\hline Fusobacterium nucleatum subsp. vincentii & DSM 19507 & EM48 & Human periodontal pocket \\
\hline Leptotrichia buccalis & DSM 1135 & C-1013-b & Supragingival calculus \\
\hline Rothia dentocariosa & DSM 43762 & CDCX599 & Carious teeth \\
\hline Rothia mucilaginosa & DSM 20746 & $5762 / 67$ & Throat \\
\hline Selenomonas sputigena & DSM 20758 & VPI D 19B-28, VPI D19B-28 & Subgingival sulcus \\
\hline Streptococcus anginosus & DSM 20563 & Havill III. (R. Lancefield F68A) & Human throat \\
\hline Streptococcus infantis & DSM 12492 & GTC $849,0-122$ & Human tooth surface \\
\hline Streptococcus intermedius & DSM 20573 & 1877, VPI 3372A & $\mathrm{N} / \mathrm{A}$ \\
\hline Streptococcus mitis & & HSISM1 & Human ileostomy \\
\hline Streptococcus oralis & DSM 20627 & LVG/I, PB182 & Human mouth \\
\hline Veillonella dispar & DSM 20735 & ERN & Human mouth \\
\hline Veillonella parvula & & HSIVP1 & Human ileostomy \\
\hline Porphyromonas catoniae & ATCC 51270 & VPI N3B-3 & Human gingival crevice \\
\hline Porphyromonas gingivalis* & & W83 & Human oral infection \\
\hline Prevotella melaninogenica & DSM 7089 & B282, VPI 4196 & Sputum \\
\hline
\end{tabular}

* Included as a reference strain. N/A: Not assigned.

\section{Preparation of bacterial treatments}

The bacteria were grown until stationary phase under the conditions and culture media recommended by DSMZ (https:// www.dsmz.de), after which glycerol ( $15 \% \mathrm{v} / \mathrm{v})$ stocks were prepared and stored at $-80^{\circ} \mathrm{C}$ until further use. On the day of the experiment, the bacterial stocks were spun down by centrifugation $(4,000 \mathrm{xg}, 7$ minutes at room temperature) and the spent media were removed. The bacteria were re-suspended in FCS-free DMEM without antibiotics to obtain the original optical density at $600 \mathrm{~nm}(0 \mathrm{D} 600)$. The samples were further diluted to obtain a multiplicity of infection (MOI) of 100 and 500 using a generalized conversion rate of 0 D600 of 1 corresponding to approximately $5 \times 10^{8} \mathrm{CFU} / \mathrm{ml}$.

\section{Scratch assay}

The scratch assay was carried out as described previously ${ }^{155}$. Briefly, cells were seeded in 96-well plates (BD Falcon ${ }^{\mathrm{TM}}$, Corning, NY, USA) at a density of $3.5 \times 10^{4}$ cells/well and incubated overnight. The cells were starved for 2 hours in FCS-free DMEM and fluorescently labelled with $2 \mu \mathrm{g} / \mathrm{ml}$ Hoechst 33342 (Molecular Probes, OR, USA) and $2 \mu \mathrm{M}$ (ellTracker ${ }^{\mathrm{TM}}$ Red 
CMTPX (Molecular Probes). The cell monolayers were simultaneously scratched with the HTS Scratcher (Peira, Antwerpen, BE) and washed twice with phosphate-buffered saline (PBS). The bacterial treatments were immediately added into the wells and screened for their effect on re-epithelialization. The positive control consisted of $4 \mathrm{ng} / \mathrm{ml}$ human transforming growth factor a (hTGFa; R\&D Systems, MN, USA). FCS-free DMEM was included as non-treated control and a combination of p38 (SB203580; Cell Signaling Technology, MA, USA) and MEK1/2 inhibitors (U0126, Cell Signaling) at a concentration of $10 \mu \mathrm{M}$ each served as negative control. The kinetics of the re-epithelialization process was extracted and quantified as described previously 155,212 . The number of cells infiltrating into the scratched area over time was modelled by a nonlinear least squares regression of the modified Gompertz function ${ }^{105}$. This procedure determines the $\mu_{\mathrm{m}}$ (repair rate, cells minute ${ }^{-1}$ ) and $A$ (maximum number of cells in the scratched area) parameters, which were also used in combination as a measure of overall performance value $\left(\mu_{\mathrm{m}}{ }^{*} \mathrm{~A}\right)$, collectively describing the efficiency of the re-epithelialization process. The overall quality of each assay run was assessed by calculation of the $Z$ ' factor, which is based on the ratio between the variability of the controls and the absolute difference between the means of the positive and negative controls ${ }^{106}$.

\section{Association of oral bacterial species with gingival bleeding during the development of gingivitis}

To evaluate the relation of the selected species and species clusters (see Table 1) with gingival bleeding during the development of mild gingivitis, we searched for the same species assignments in two niches (i.e. supragingival plaque, and unstimulated saliva) of individuals participating in an experimental gingivitis study ( $n=41$ ) based on the V4 hypervariable region of their $16 \mathrm{~S}$ rRNA gene (100\% identity, 100\% coverage). The sequence data was subsampled to equal depth (10,000 reads/sample) and log transformed to normalize their distribution. The normalized read counts determined at the beginning (Day 0 ) and at the peak of the challenge (Day 14) were related to the percentage of gingival bleeding at the respective timepoints by a redundancy analysis (RDA) $)^{215}$ using Canoco version 5.0 $0^{216}$. To evaluate if the increase or decrease of relative abundance of the selected species was related to the magnitude of the response to the challenge, we performed an RDA using the difference between the read counts (sample-wise and zero-mean scaled) at the peak and the baseline of the challenge ( $\Delta$ relative counts) in relation to the change in gingival bleeding ( $\triangle \mathrm{BOMP} \%$ ). The RDAs performed were carried out with a total of 499 permutations. Spearman correlation analyses were carried out using GraphPad version 6.0 (GraphPad Software, CA, USA).

\section{Other statistical analysis}

Statistical differences were determined using GraphPad version 6.0 with a limit of significance set at $P<0.05$. Differences between groups were assessed by a two-tailed Mann-Whitney $U$ test, whereas comparison between the different treatments and the non-treated control were assessed by a one-way ANOVA. 


\section{Results}

\section{Identification of oral bacteria related to higher or lower gingival bleeding scores}

In a previously reported cross-sectional observational study on 268 systemically healthy individuals, we determined the microbiota composition in seven niches of their oral cavity, i.e. interproximal plaque, subgingival lower jaw, subgingival upper jaw, supragingival, tongue posterior, tongue anterior, and unstimulated saliva ${ }^{211}$. The percentage of gingival bleeding on marginal probing (BOMP) and on pocket probing (BOPP) were also recorded for each individual in the study ${ }^{177}$. In an effort to identify microbial genera positively or negatively associated with gingival inflammation, we employed elastic net regression ${ }^{178}$ with stability selection ${ }^{179}$ using the percentages of gingival bleeding and the oral microbiome datasets (see Materials and Methods for more details) derived from the volunteers that participated in the study. The positive or negative fractional weight values (Table S1) determined the direction of the association of specific operational taxonomic units (OTUs) with the gingival bleeding. A very high stability ( $\geq 84$ ) was assigned to all of the identified OTUs (Table S1), indicating that these OTUs were selected in the majority of the iterations $(n=100)$ performed. The selected 0TUs were first examined at genus-level (Table S1) and the frequency at which specific genera were associated with a higher and lower percentage of gingival bleeding was determined (Fig. 1). Genera represented by a single 0TU were excluded from further analyses (Table S1).

The elastic net regression with stability selection was able to identify genera that were consistently associated with higher (i.e. Fusobacterium, Capnocytophaga, Porphyromonas, Treponema, Eubacterium, Peptococcus, Peptostreptococcaceae, Selenomonas, and Tannerella) or lower (i.e. Corynebacterium and Veillonella) gingival bleeding scores (Fig. 1). In addition, Leptotrichia was mostly associated with a higher percentage of gingival bleeding (14 0TUs vs 3 0TUs), whereas Rothia was mainly associated with a lower percentage of gingival bleeding (7 OTUs vs 1 OTU). Prevotella, Streptococcus and Actinomyces displayed similar number of OTUs associated with gingival bleeding in either direction. Therefore, to further refine their association with gingival bleeding, the representative sequences of these OTUs were examined at species-level (Table S2). Several 0TUs identified as Prevotella melaninogenica were strongly associated with both higher (fractional weight of 0.35 and 0.44 ) and lower (fractional weight of -0.49 ) gingival bleeding scores, which may be a result of stain-specific effects. The Streptococcus and Actinomyces OTUs could not be assigned at species level due to insufficient phylogenetic resolution within the region of the 165 rRNA gene that was analysed. Therefore, the representative sequences of these OTUs were assigned to a species-cluster with identical percentage of similarity. The OTUs assigned to the streptococcal group Streptococcus oralis/dentisani/tigurinus/mitis/infantis were both positively and negatively associated with gingival bleeding, which could correspond to species-specific effects on gingival cells. Conversely, the OTUs assigned to Streptococcus intermedius/anginosus and Actinomyces oris/viscosus/naes/undii were consistently found to be positively and negatively associated with gingival bleeding, respectively (Table S2). The 150 TUs with the strongest and most stable positive and negative association (selected from Table S1) with gingival bleeding were assigned a species or species-cluster (i.e. in cases where the OTU sequence could not be assigned to a single species) name (Table 2). 


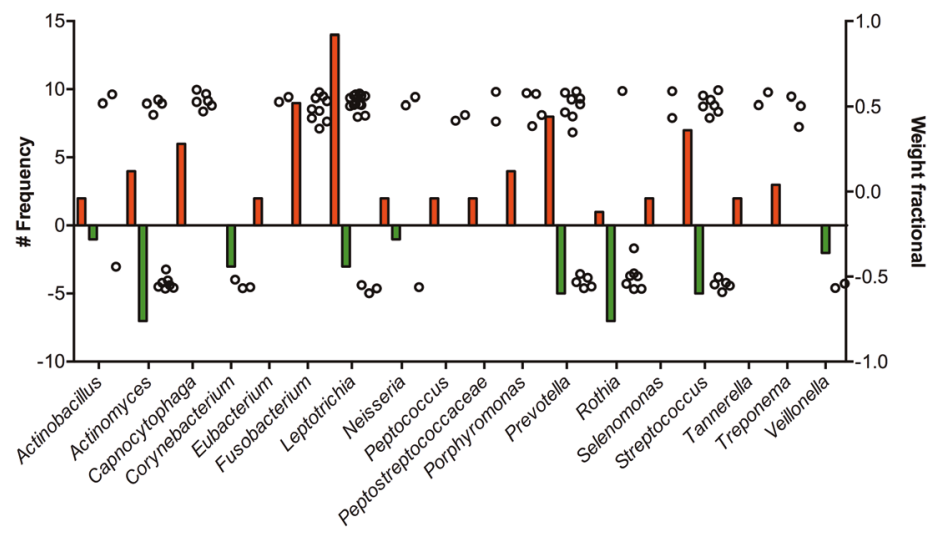

Figure 1. Identification OTUs associated with gingival bleeding. Frequency and fractional weight values obtained for the identified operational taxonomic units (OTUs) at genus-level. Red and green bars represent the frequency at which specific genera were positively or negatively associated with gingival bleeding, respectively. Open circles represent the fractional weight obtained for each of the selected OTUs.

Table 2. Species assignment. Species or species-cluster names assigned to the 15 OTUs with the strongest and most stable negative and positive association with gingival bleeding as determined by the fractional weight. Percentage similarity of the $16 \mathrm{~S}$ rRNA sequence to the database, prevalence of the 0TU within the study cohort, and niche in which they were identified by elastic net are also indicated (US, $n=267 ; \mathrm{PL}, \mathrm{n}=258, \mathrm{PI}, \mathrm{n}=248$ ).

\begin{tabular}{|c|c|c|c|c|c|}
\hline OTU & Niche* $^{*}$ & Fractional weight & Species name & Identity (\%) & Prevalence (\%) \\
\hline 0tu0532 & US & -0.5416 & Veillonella atypica/dispar/parvula & 97.6 & 40.45 \\
\hline Otu0376 & PL & -0.5369 & Actinomyces oris/viscosus/naeslundii & 99.6 & 69.38 \\
\hline 0tu0174 & US & -0.5359 & Streptococcus oralis/dentisani/tigurinus/mitis/infantis & 99.6 & 96.25 \\
\hline Otu0300 & $\mathrm{PL}$ & -0.5322 & Prevotella oulorum & 99.2 & 13.95 \\
\hline 0tu0007 & $\mathrm{PL}$ & -0.5229 & Actinomyces oris/viscosus & 100.0 & 100 \\
\hline Otu0054 & $\mathrm{PL}$ & -0.5185 & Corynebacterium durum & 100.0 & 98.84 \\
\hline Otu0490 & $\mathrm{PI}$ & -0.5072 & Prevotella oulorum & 100.0 & 69.23 \\
\hline 0tu0004 & US & -0.5028 & Streptococcus oralis/dentisani/tigurinus/mitis/infantis & 100.0 & 100 \\
\hline Otu0534 & US & -0.4977 & Rothia dentocariosa & 98.8 & 12.36 \\
\hline 0tu0606 & $\mathrm{Pl}$ & -0.4963 & Rothia dentocariosa & 98.8 & 30.36 \\
\hline Otu0021 & PL & -0.4851 & Prevotella melaninogenica & 100.0 & 76.36 \\
\hline Otu0808 & US & -0.4808 & Rothia dentocariosa & 98.0 & 6.74 \\
\hline Otu0287 & $\mathrm{PL}$ & -0.4579 & Actinomyces oris/viscosus/naeslundii & 99.6 & 78.68 \\
\hline Otu0219 & US & -0.4411 & Actinobacillus porcinus/rossi & 100.0 & 78.28 \\
\hline Otu0108 & $\mathrm{PL}$ & -0.3325 & Rothia mucilaginosa & 99.6 & 19.77 \\
\hline Otu0139 & $\mathrm{PL}$ & 0.3470 & Prevotella melaninogenica & 99.6 & 25.58 \\
\hline Otu0005 & PL & 0.3695 & Fusobacterium nucleatum subspecies animalis & 100.0 & 98.45 \\
\hline Otu0256 & PL & 0.3799 & Treponema lecithinolyticum & 100.0 & 18.60 \\
\hline Otu1021 & PL & 0.3843 & Porphyromonas catoniae & 97.6 & 32.17 \\
\hline Otu0237 & $\mathrm{PL}$ & 0.3969 & Johnsonella ignava & 100.0 & 39.15 \\
\hline Otu0274 & PL & 0.4121 & Eubacterium yurii & 100.0 & 70.93 \\
\hline Otu0311 & $\mathrm{PL}$ & 0.4123 & Fusobacterium nucleatum subspecies nucleatum & 98.0 & 47.29 \\
\hline Otu0223 & US & 0.4162 & Peptococcus sp. oral taxon & 100.0 & 17.23 \\
\hline 0tu0006 & PL & 0.4311 & Fusobacterium nucleatum subspecies vincentii/fusiforme & 100.0 & 99.61 \\
\hline Otu0074 & US & 0.4326 & Selenomonas sputigena & 100.0 & 34.08 \\
\hline Otu0617 & $\mathrm{PL}$ & 0.4330 & Streptococcus intermedius/anginosus & 98.8 & 38.37 \\
\hline Otu0512 & PL & 0.4388 & Leptotrichia buccalis & 98.8 & 25.97 \\
\hline Otu0118 & PL & 0.4409 & Prevotella melaninogenica & 99.6 & 31.40 \\
\hline Otu0077 & $\mathrm{PL}$ & 0.4459 & Leptotrichia buccalis & 100.0 & 86.05 \\
\hline $0 \mathrm{tu0} 0223$ & PL & 0.4503 & Peptococcus sp. oral taxon & 100.0 & 56.59 \\
\hline
\end{tabular}

* Niche: (US) unstimulated saliva, (PL) supragingival plaque, (PI) interproximal plaque. 
The participants with gingival bleeding scores (i.e. BOMP and BOPP) below the average of the entire cohort were classified as individuals with "lower bleeding", whereas the participants with scores above that average were classified as individuals with "higher bleeding". When we examined the relative abundance of the selected OTUs (Table 2 ) in these two groups, we were able to confirm that the OTUs that were found to be negatively associated with gingival bleeding by elastic net regression were on average more abundant in the individuals that displayed lower gingival bleeding scores (Fig. 2, also see Fig. S1). In contrast, the OTUs with the strongest positive association with gingival bleeding were on average more abundant in the individuals with higher gingival bleeding. In particular, a highly significant difference between the groups $(P<0.0001)$ was found for 0TU0287 and 0TU0005, which were assigned to Actinomyces oris/viscosus/naes/undii and Fusobacterium nucleatum subspecies animalis, respectively.

a. Unstimulated saliva
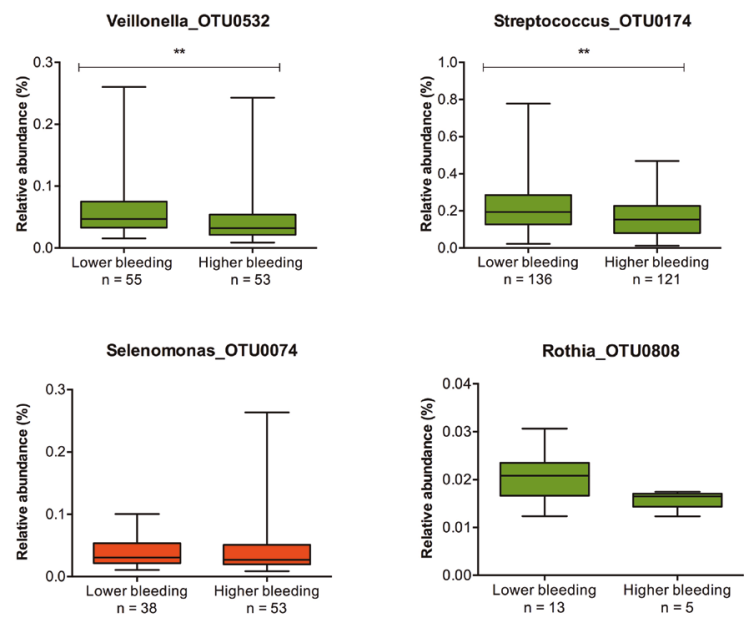

b.

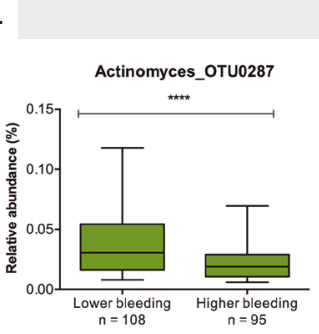

$$
\text { Plaque supragingival }
$$
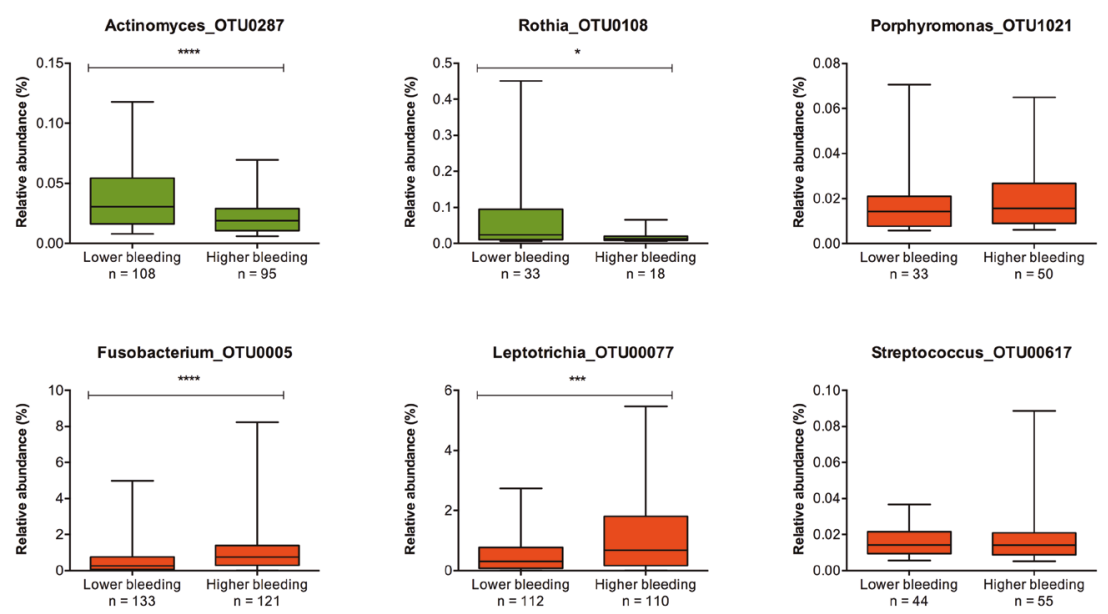

Figure 2. Relative abundance and prevalence of the selected OTUs in relation to gingival bleeding. (a) Representative graphs of the selected OTUs in unstimulated saliva. (b) Representative graphs of the selected OTUs in supragingival plaque. Significant differences between groups were assessed by a two-tailed Mann-Whitney $U$ test $\left({ }^{*}, \mathrm{P}<0.05 ;{ }^{* *}, \mathrm{P}<0.01,{ }^{* * *}, \mathrm{P}<0.001 ;{ }^{* * * *}, \mathrm{P}<0.0001\right)$. Green boxplots represent 0 TUs found to be negatively associated with gingival bleeding and red boxplots represent $0 T$ S found to be positively associated. 


\section{Oral species associated with gingival bleeding and their influence in re-epithelialization}

Prompt re-epithelialization of damaged oral mucosa is considered essential for maintenance of a healthy state of the oral cavity. We hypothesized that the association of the identified oral bacteria with gingival bleeding could be related to their influence in gingival re-epithelialization and therefore, we decided to evaluate this proposed host-microbe interaction using a recently developed in vitro scratch assay to determine the kinetics of the wound healing process ${ }^{155}$. To this end, based on the frequency at which species or species-clusters were found to be positively or negatively linked to gingival bleeding as well as the strength and consistency of these associations, we obtained representative strains of the assigned species (Table 1). Gingival epithelial cells were exposed to a multiplicity of infection (MOI) of 100 and 500 of these strains (Fig. 3). As a negative control, we included the periodontal pathogen Porphyromonas gingivalis ${ }^{207}$ strain W83 which has been previously shown to strongly inhibit in vitro re-epithelialization of oral epithelial cells $s^{7980,155}$.

The repair rate represented by the $\mu_{\mathrm{m}}$ parameter was calculated by fitting the modified Gompertz function through the reepithelialization measurements ${ }^{105}$. Notably, A. oris, A. viscosus and V. parvula, which were all predicted to have a consistent negative association with gingival bleeding, were among the strongest stimulators of the repair rate irrespective of the dosage used (Fig. 3a, b). Likewise, the Rothia' species had a neutral effect on re-epithelialization at any of the dosages tested, whereas increasing concentrations of $V$. dispar and $A$. naeslundii resulted in a higher repair rate when compared to the non-treated control. The species that were identified as positively associated with gingival bleeding, elicited somewhat more variable effects in the re-epithelialization assay. For instance, treatment with $F$. nucleatum subsp. animalis at a $\mathrm{MO}$ of 100 significantly accelerated re-epithelialization kinetics when compared to the non-treated control (Fig. 3a), but treatment with the same strain at an MOI 500 resulted in strong inhibition of the healing process (Fig. 3b). The inhibitory effect elicited by $F$. nucleatum subsp. animalis was comparable to that exerted by $P$. gingivalis W83, which was included here as negative control. Analogously, S. sputigena only attenuated the re-epithelialization kinetics at the higher dosage tested, whereas F. nucleatum subsp. nucleatum and subsp. vincentii already decreased the repair rate already at the lower $\mathrm{MOI}$ tested. Nevertheless, irrespective of the dosage used some strains like S. anginosus and $P$. catoniae consistently decreased or increased the repair rate as compared to the non-treated control, respectively. Remarkably, when we compared the overall re-epithelialization performance $\left(\mu_{\mathrm{m}}{ }^{*} \mathrm{~A}\right.$; see Materials and Methods for explanation) value of the group of strains associated with lower or higher gingival bleeding, the group of commensal bacteria negatively associated with gingival bleeding elicited significantly increased re-epithelialization kinetics than the positively associated group at any of the dosages tested (Fig. 3c, d). 
MOI 100

a.

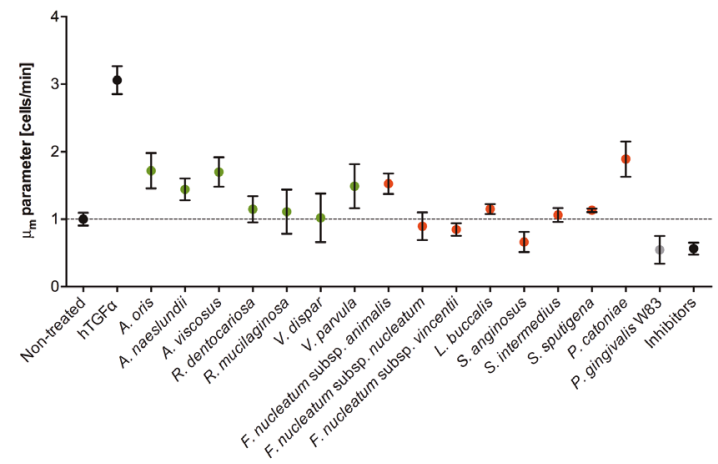

MOI 500

b.

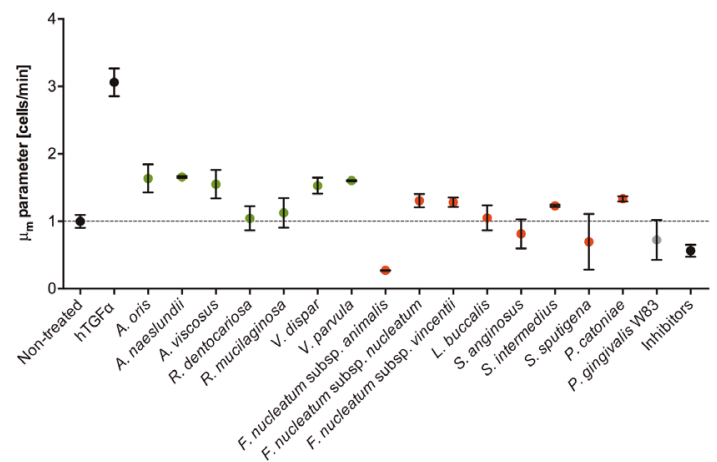

c.

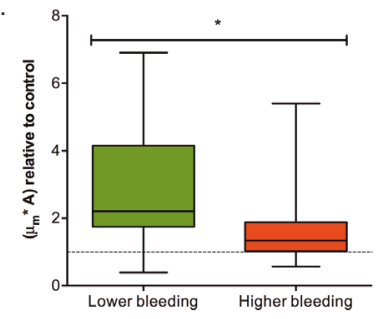

d.

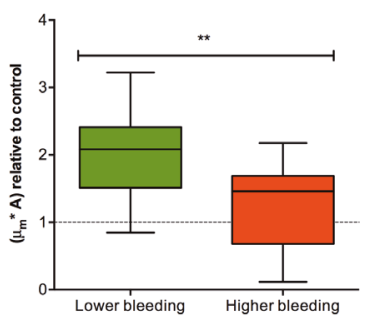

Figure 3. Effect of representative oral commensal bacteria on gingival re-epithelialization. Repair rate ( $\mu_{\mathrm{m}}$ parameter) of gingival cells upon treatment with representative oral strains at MOI 100 (a) or 500 (b). Comparison between groups of bacteria associated with lower or higher gingival bleeding at MOI 100 (c) or 500 (d). Human transforming growth factor a (hTGF a, $4 \mathrm{ng} / \mathrm{ml}$ ) was used as positive control, whereas a combination of inhibitors of $p 38$ and MEK $1 / 2$ served as negative control (10 $\mu \mathrm{M}$ each). Significant differences between groups were assessed by a two-tailed MannWhitney $U$ test $\left({ }^{*}, P<0.05 ;{ }^{* *}, P<0.01\right)$. Species positively associated with gingival bleeding are represented in red; and species negatively associated are shown in green. Dotted lines represent the effect of the non-treated control.

Finally, representative strains of the species clustered under the S. oralis/dentisani/tigurinus/mitis/ infantis group that was both positively and negatively associated with gingival bleeding, had opposing effects on re-epithelialization kinetics (Fig. S2) which is in agreement with the suggested species-specific effects within this group. The impact of $P$. melaninogenica on re-epithelialization was dependent on the dosage used (Fig. S2). However, as concluded above the impact of this species may be highly strain dependent and thus, a clear conclusion for this species cannot be drawn. 


\section{Validation of the role of the identified species during an experimental gingivitis challenge}

To evaluate if the selected representative species play a role in the development of mild gingivitis, we evaluated the relationship between the abundance of these bacteria and the gingival bleeding scores recorded for the participants in an experimental gingivitis challenge study ${ }^{211}$. The experimental gingivitis challenge was carried out with healthy volunteers $(n=41)$ who were instructed to refrain from any form of oral hygiene for a period of two weeks. Microbiota analyses were performed at the start (Day 0 ) and at the peak of the challenge (Day 14). Based on the 165 rRNA gene sequence, we searched for the selected species or species-clusters in the samples derived from unstimulated saliva and supragingival plaque, the two main niches in which the OTU associations with gingival bleeding were originally identified (Table 2). Since most of the OTUs identified by elastic net regression in the first study were present in both niches and appeared to maintain a similar relationship between the individuals with higher and lower gingival bleeding scores (Fig. S3), we conducted a redundancy analyses (RDA) to determine the association of the relative abundance of the selected species with the gingival bleeding on marginal probing (BOMP), independently of the niche in which they were originally identified (Fig. 4).

At the start of the challenge, the variation in the relative abundance of the selected species in either of the niches examined (i.e. supragingival plaque and unstimulated saliva) was not significantly explained by the gingival bleeding measurements recorded for each individual (Fig. S4). This could be due to the relatively low bleeding scores of these individuals in combination with the small size cohort $(n=41)$ that may be insufficient to detect similar relationships as those we identified in the larger cross-sectional study $(n=268)$. However, as we conducted the RDA at the peak of the challenge (Day 14), $6.1 \%$ of the total variation (3.7\% adjusted explained variation) in unstimulated saliva could be significantly explained ( $P=0.016$ ) by the exacerbated gingival bleeding scores within the cohort (Fig. $4 a$ ). Importantly, the RDA analysis does not take into account the positive or negative gingival bleeding-association of individual bacterial species. Nevertheless, overlaying the RDA with the gingival bleeding relationships established by the elastic net regression distributed the bacterial species along the gingival bleeding axis in accordance with the elastic net results, which significantly strengthens their proposed association with gingival bleeding. In particular, higher relative abundances of the representative sequences identified as A. oris/viscosus/naes/undii (ID_5a and ID_5b), R. dentocariosa (ID_12), and V. dispar (ID_10a) had a strong negative association with gingival bleeding, whereas P. catoniae (ID_6), S. sputigena (ID_13), and S. anginosus (ID_15) were found to be associated with increased gingival bleeding. Analogously, when conducting the same analysis in supragingival plaque (Fig. $\mathbf{4 b}$ ), despite not being significant according to the 95\% confidence level $(P=0.078)$, the associations of the selected bacteria with the gingival bleeding scores were highly congruent with the previously established relationships. 
a.

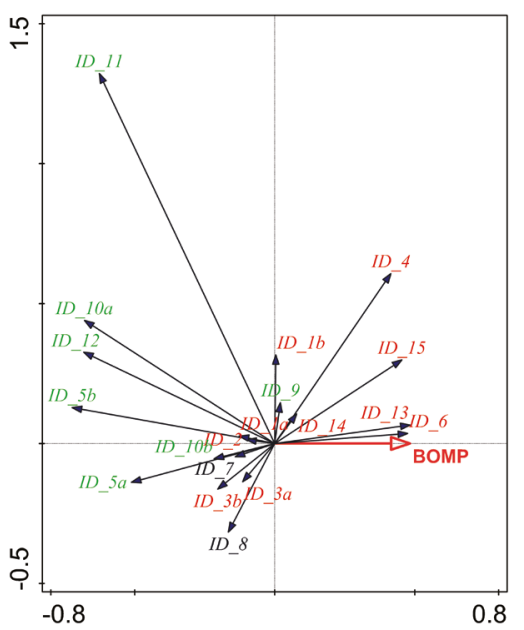

b.

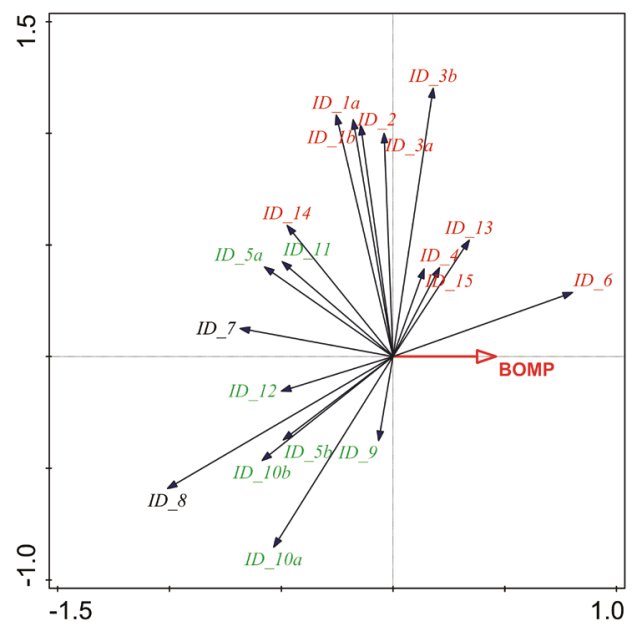

ID_1a Fusobacterium nucleatum subspecies animalis

ID_1b Fusobacterium nucleatum subspecies animalis

ID 2 Fusobacterium nucleatum subspecies nucleatum

ID 3 a Fusobacterium nucleatum subspecies vincentii

ID_3b Fusobacterium nucleatum subspecies vincentii

ID 4 Leptotrichia buccalis

ID 5 a Actinomyces oris/viscosus/naes/undii

ID_5b Actinomyces oris/viscosus/naeslundii

ID_6 Porphyromonas catoniae

ID 7 Streptococcus oralis/dentisani/tigurinus/mitis/infantis

ID_8 Prevotella melaninogenica

ID_9 Veillonella parvula

ID 10a Veillonella dispar

ID $10 \mathrm{~b}$ Veillonella dispar

ID_11 Rothia mucilaginosa

ID_12 Rothia dentocariosa

ID 13 Selenomonas sputigena

ID 14 Streptococcus intermedius

ID_15 Streptococcus anginosus

Figure 4. RDA analysis of the relationship of selected species and species-clusters with gingival bleeding at the peak of the experimental gingivitis challenge. (a) Relative abundance of the selected species in unstimulated saliva explained by the BOMP at the peak of the challenge ( $P=$ 0.016). (b) Relative abundance of the selected species in supragingival plaque explained by the BOMP at the peak of the challenge $(P=0.078)$. Species found to be negatively associated with gingival bleeding scores in the cross-sectional study by elastic net regression are represented with green labels, whereas positive associations are presented with red labels. Black labels represent bacterial species that were both positively and negatively associated with gingival bleeding. Each arrow in the RDA points in the direction of the steepest increase of the values for the corresponding species (species-cluster).

\section{Experimental gingivitis responsiveness in the context of microbial composition and dynamics}

To explore whether the increase or decrease of relative abundance of particular species was associated with a stronger response (i.e. higher increase in gingival bleeding) to the experimental gingivitis challenge, we conducted an RDA using the difference in relative abundance ( $\Delta$ relative counts) in relation with the change in gingival bleeding $(\triangle B O M P \%)$ between the peak and the beginning of the challenge in unstimulated saliva samples (Fig $5 \mathrm{a}$ ). In addition, the strongest relationships identified by this RDA were also examined by Spearman correlation analyses. Taken together, these results showed a significant positive correlation between the change in relative abundance of S. sputigena (ID_13) and the response to the challenge $(r=0.44, P=0.01, n=32)$ (Fig $5 \mathbf{b})$. In contrast, an increase in the relative abundance of $V$. 
dispar (ID_10a) had a significant protective effect in the challenge $(r=-0.35, P=0.027, n=41)$ and corresponded to a reduced change in gingival bleeding (Fig $\mathbf{5 C}$ ). Although the Spearman correlation of the RDA-identified associations of the changes in relative abundance of other individual bacterial species did not meet statistical significance, the direction of their association with the change in gingival bleeding followed the prediction made by the elastic net analysis (Fig. S5). In particular, the results suggest that a reduction of the relative abundance of other potential beneficial species such as A. oris/viscosus/naes/undii (ID_5a; $r=-0.25, P=0.16, n=33$ ) and V. parvula (ID_9; $r=-0.19, P=0.23, n=41$ ) corresponded with larger changes in gingival bleeding during the challenge, while an opposite relation was seen for $F$. nucleatum subsp. nucleatum (ID_2; $r=0.33, P=0.16, n=19$ ) and $S$. anginosus (ID_15; $r=0.21, P=0.30, n=25$ ) (Fig. S5). Only Rothia mucilaginosa (ID_11) appeared to be an exception as it was initially identified as negatively associated with gingival bleeding (Table 2), while during the experimental gingivitis challenge a relatively small increase in gingival bleeding was related to a reduction of the relative abundance of this species $(r=0.57, P=0.002, n=28$; Fig. S5).

a.

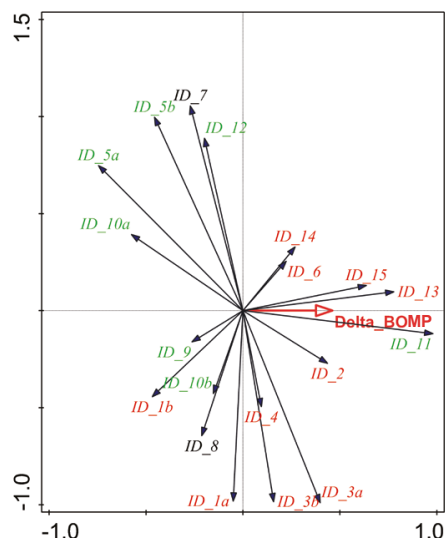

b.

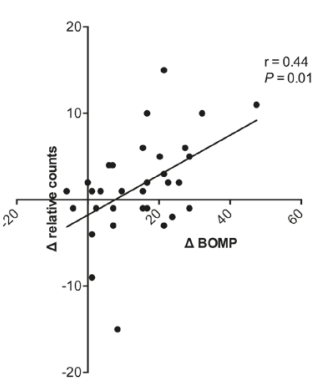

c.

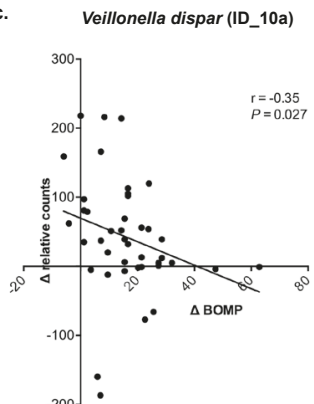

d.
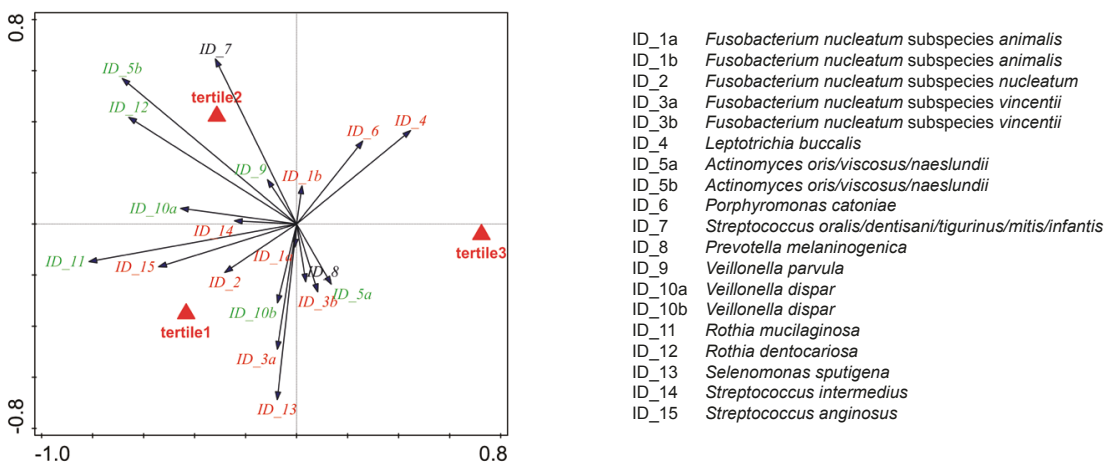

Figure 5. Experimental gingivitis responsiveness in the context of microbial composition and dynamics. (a) Change in relative abundance ( $\triangle$ relative counts) between the peak (Day 14 ) and the beginning (Day 0$)$ of the challenge explained by the change in gingival bleeding $(\triangle B 0 M P \%)$ $(\mathrm{P}=0.048)$, analysed by redundancy analysis (RDA). (b) Association between the change in relative abundance of $S$. sputigena and (c) Veillonella dispar and the $\triangle B O M P \%$ assessed by Spearman correlation analyses. (d) Relative abundance at Day 0 of the selected species in unstimulated saliva samples explained by $\triangle B O M P \%$ represented in tertiles (tertile 1, small change; tertile 2, medium change; tertile 3, large change) (explained variation $8.5 \%$, $P=0.048)$. 
Finally, we evaluated whether the relative abundance of the selected species and species-clusters at the beginning of the challenge period (Day 0 ) could be employed to predict the magnitude of the individual's response to the experimental gingivitis challenge, which was reflected by the change in gingival bleeding (Fig $\mathbf{5 d}$ ). Remarkably, redundancy analysis showed that the tertiles of lowest, medium and highest changes in BOMP during the trial could explain $8.5 \%$ of the variation in relative abundance of the selected bacterial groups at Day $0(P<0.05)$, illustrating that microbial signatures composed of specific bacteria may be of diagnostic value to predict disease risk.

\section{Discussion}

The oral cavity provides a warm and moist environment that supports the growth and colonization of a distinctive collection of microorganisms ${ }^{217}$. The composition of the oral ecosystem is relatively stable and in healthy conditions, the resident microbes have a harmonious and symbiotic relationship with the hos $\mathrm{s}^{33,218}$. However, the stability of the oral ecosystem can be disrupted by a substantial change in a parameter that is considered critical for maintenance of homeostasis, such as adequate hygiene measures. This may result in a microbial shift where certain microorganisms subvert host surveillance, increase in relative abundance, and ultimately contribute to the initiation of disease $e^{219,220}$. Such microbial dysbiosis in the oral cavity can lead to the development of periodontal diseases, which encompass the common inflammatory disorders of gingivitis and periodontitis 221 . Periodontal diseases, apart from being detrimental to oral health, favour the entry of bacteria into the submucosal connective tissues and the bloodstream, which pose a direct risk factor for the development of focal infections such as infective endocarditis ${ }^{2}$. Moreover, active periodontitis have been associated with the development of atherosclerosis ${ }^{3}$, increased risk for gestational diabetes ${ }^{51}$ and pre-term low weight birth ${ }^{52}$.

Porphyromonas gingivalis and Fusobacterium nucleatum among other gram-negative bacteria are frequently isolated from infected periodontal pockets and therefore, have been regarded as periodontal pathogens ${ }^{222}$. In vitro studies using oral epithelial cells have shown that upon disruption of a confluent monolayer of cells, $P$. gingivalis and to a lesser extent $F$. nucleatum can adversely affect re-epithelialization through induction of apoptosis, and a reduction of cell migration and proliferation ${ }^{79,80,155}$. However, the role of other commensal bacteria on re-epithelialization and their potential contribution to a healthy state of the oral cavity, remains unexplored. We used elastic net regression with stability selection to identify commensal species in the oral microbiome of 268 healthy subjects that were associated with gingival bleeding scores, a clinical parameter that reflects the inflammatory state of the periodontal tissues ${ }^{177,211}$. We hypothesized that the associations of the identified species with gingival bleeding could be related to their capacity to influence oral re-epithelialization, an essential process for maintenance of mucosal homeostasis and epithelial barrier function ${ }^{7}$. Representative species were screened in vitro for their capacity to influence re-epithelialization kinetics $\left(\mu_{m}{ }^{*} A\right)^{155}$, showing that the groups of bacteria negatively associated with gingival bleeding on average enhanced reepithelialization kinetics more than the positively associated group of bacteria. In particular, A. oris, A. viscosus and V. parvula, consistently promoted the repair rate at the two bacterial dosages tested (i.e. MOI 100 and 500). Interestingly, previous reports already proposed that Veillonella and Actinomyces are negatively correlated to clinical parameters related to periodontitis ${ }^{223,224}$. Moreover, a recent study that pooled the sequencing data from several published studies (i.e. 196 subgingival samples from patients with chronic periodontitis and 422 subgingival samples from healthy individuals) aiming to detect genera within the oral microbiota that are specific to health and disease, identified a higher prevalence of Veillonella and Actinomyces in healthy individuals ${ }^{225}$. 
Among the positively associated bacteria identified by elastic net regression, F. nucleatum subsp. nucleatum, vincentii and animalis elicited opposing effects on in vitro re-epithelialization depending on the dosage used. Specifically, exposure of gingival cells to $F$. nucleatum subsp. animalis highly stimulated repair rate at the lower dosage tested (MOI 100), but a 5-fold higher dosage led to strong inhibition of the healing process, which was comparable to the effect exerted by the negative controls, including P. gingivalis. Similarly, increasing concentration of Selenomonas sputigena decreased the repair rate in comparison with the non-treated control. Different studies have shown the association of Fusobacterium, Porphyromonas and Selenomonas with subgingival samples derived from subjects suffering from chronic periodontitis ${ }^{226-228}$. Notably, F. nucleatum subsp. polymorphum and vincentii were shown to be also associated with oral squamous cell carcinoma $(0 \mathrm{SCC})^{229}$. Interestingly, the microbial genes and pathways enriched in 0SCC samples were of pro-inflammatory nature and very similar to those identified in association with chronic periodontitis ${ }^{226}$. Intriguingly, $S$. anginosus, identified in this study as positively associated with gingival bleeding, has been frequently found in cancer samples derived from the oesophagus ${ }^{230,231}$, stomach ${ }^{231}$, and neck squamous carcinomas ${ }^{232}$.

The salivary microbiome results from microbiota that shed from different oral niches and has been shown to be representative of the overall oral microbiome ${ }^{233}$. In this study, we were able to identify highly consistent associations between specific species in unstimulated saliva samples and the periodontal health status of the subjects from two different cohorts using elastic net regression and redundancy analyses, respectively. Moreover, when we examined how the change in relative abundance of these species influenced the individuals' response to the experimental gingivitis challenge, we found that an increased proportion of $S$. sputigena was positively correlated with an increased change in gingival bleeding, or responsiveness, during the challenge. In contrast, increased relative abundance of $V$. dispar was correlated with a reduced change in gingival bleeding, contributing to the stability of those individuals in response to the experimental gingivitis challenge. This finding is in line with the results obtained in a 2-year longitudinal study that explored the relationship between shifts in microbial composition in subgingival plaque and changes in periodontal health and in which Veillonella sp. was found to be significantly associated with oral health and clinically stable subjects ${ }^{224}$. The fact that in our study the association of $V$. dispar was predominantly driven by an increase in relative abundance in subjects that displayed a lower increase in gingival bleeding during the challenge, rather than by a substantial loss of $V$. dispar in subjects with a higher increase, shows that dynamics of the oral microbiota composition during disease development is more complex than the 'simple' loss of benign or gain of detrimental microbial species. Thereby, the interpretation of ecosystem dynamics in the context of disease development requires a holistic view of the microbial members within the system and their changes rather than aiming to pinpoint single 'biomarker' microbes. In addition, strain specific effects may strongly confound the association of particular species with health or disease, which we propose to be underlying the variable association of Prevotella melaninogenica with gingival bleeding. Despite the complexity of microbial ecosystem dynamics under changing conditions (e.g. healthy state towards gingivitis development) our analyses also illustrate that there are opportunities to harness ecosystem composition signatures to predict disease development risks. It should be noted that the relative abundance of individual microbes that can be classified as benign or detrimental (e.g. based on their association with low and high gingival bleeding, respectively) as such, does not appear to predict the responsiveness of the system under challenge conditions, whereas a signature composed of multiple microbes may allow such prediction.

Taken together, our study underpins that specific members of the oral microbiota can be linked to clinical parameters such as gingival bleeding in healthy and compromised (gingivitis) individuals. Moreover, some of the identified microbes can influence mucosal homeostasis by modulation of epithelial wound repair. Our study also illustrates that microbiota 
composition can be employed for the prediction of disease risk, although our understanding of this prediction in terms of ecosystem dynamics remains limited. Therefore, functional and microbial community studies to investigate the effect of increasing proportions of certain microbial groups, like V. dispar and S. sputigena, in relation to disease risk are needed. In this context, it would be of interest to evaluate the potential of certain benign commensals as probiotics to support oral health and epithelial integrity.

\section{Acknowledgements}

We thank the clinical team at the Academic Centre for Dentistry Amsterdam (ACTA) and the University of Amsterdam and Vrije Universiteit Amsterdam for planning, reviewing and executing the cross-sectional observational study as well as the experimental gingivitis challenge intervention. Furthermore, we thank Dr. Alexa M. G. A. Laheij (Academic Centre for Dentistry Amsterdam, Amsterdam, NL) and Dr. Tom van den Bogert (Laboratory of Microbiology, Wageningen University, Wageningen, NL) for providing us with P. gingivalis W83 and Veillonella parvula HSIVP1, respectively. Finally, we thank Dr. Andrei Prodan (Academic Centre for Dentistry Amsterdam, Amsterdam, NL) for facilitating the sharing of information derived from the experimental gingivitis challenge. 


\section{Supplementary data}

a.

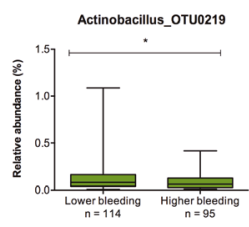

b.
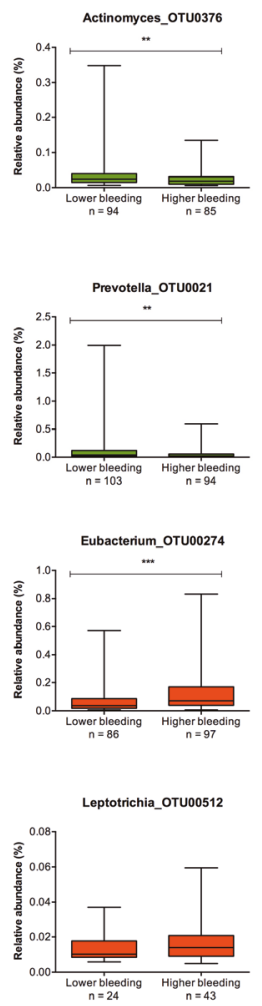

Unstimulated saliva
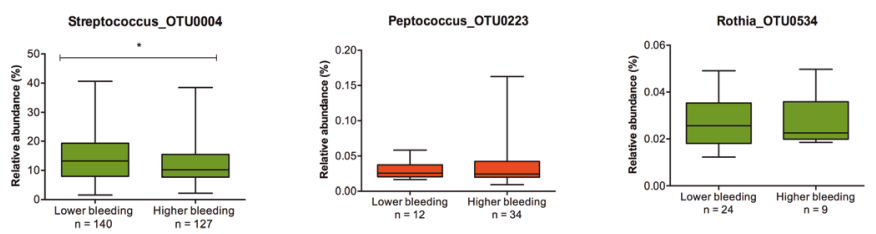

Plaque Supragingival
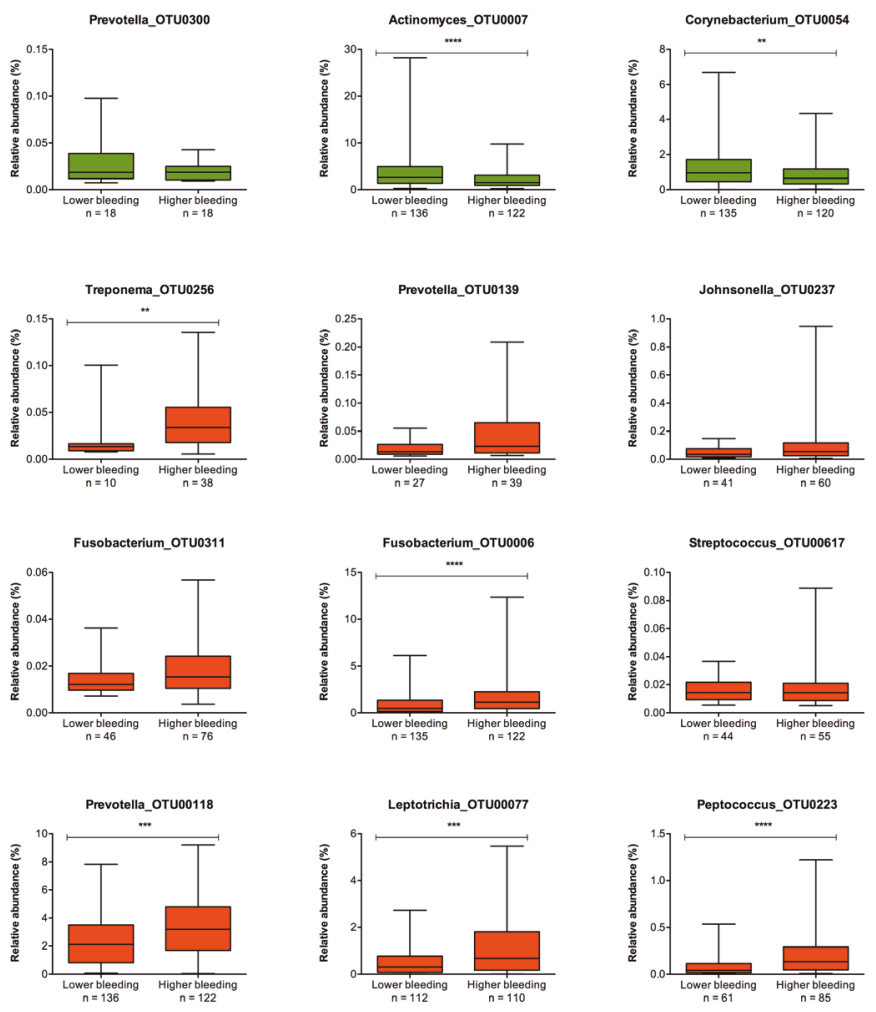

c. Plaque Interproximal
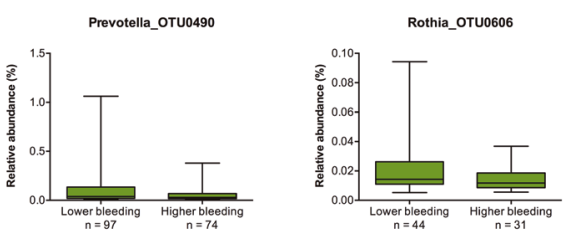

Figure S1. Relative abundance and prevalence of the 15 OTUs with the strongest positive and negative association with gingival bleeding. (a) Other selected OTUs in unstimulated saliva samples, (b) supragingival plaque, and (c) interproximal plaque. Significant differences between individuals with higher and lower gingival bleeding group were assessed by a two-tailed Mann-Whitney $U$ test $\left({ }^{*}, P<0.05\right.$; ${ }^{* *}, P<0.01$, ${ }^{* * *}$, $\left.P<0.001 ;{ }^{* * *}, P<0.0001\right)$. Green and red boxplots represent 0 TUs that elastic net analysis identified to be negatively and positively associated with gingival bleeding. 


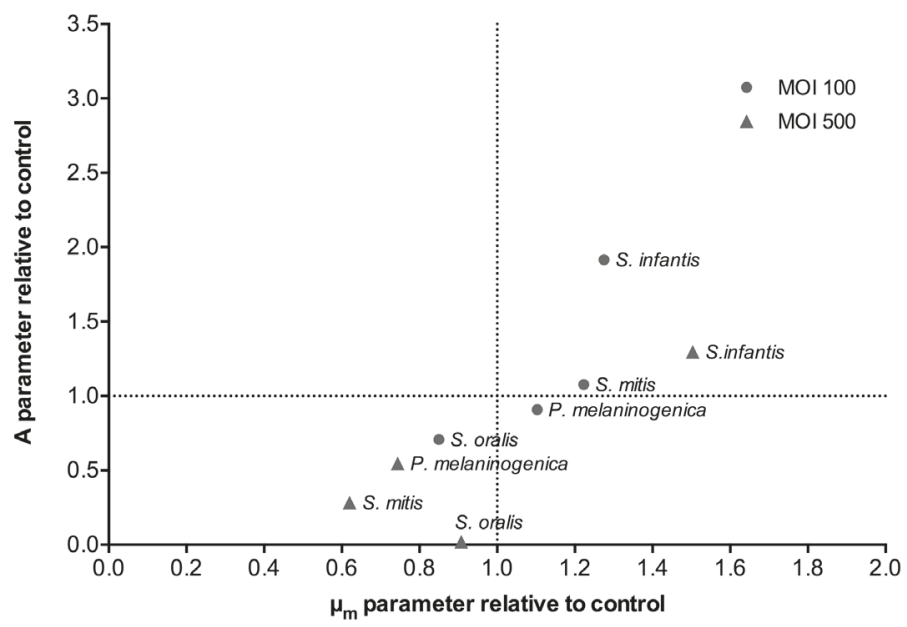

Figure S2. Relative effect of streptococcal species and P. melaninogenica on gingival re-epithelialization. S. infantis (DSM 12492), S. mitis (HSISM1), S. oralis (DSM 20627), and P. melaninogenica (DSM 7089) were tested for their influence in gingival re-epithelialization kinetics at an MOI 100 and 500. Dotted lines represent the effect of the non-treated control. The $\mu_{\mathrm{m}}$ and $\mathrm{A}$ parameters represent the repair rate and maximum number of cells in the scratched area, respectively. 
a.
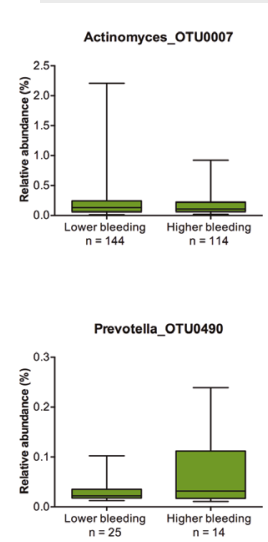

Fusobacterium_OTU0006

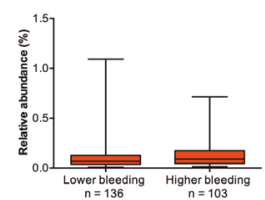

b.
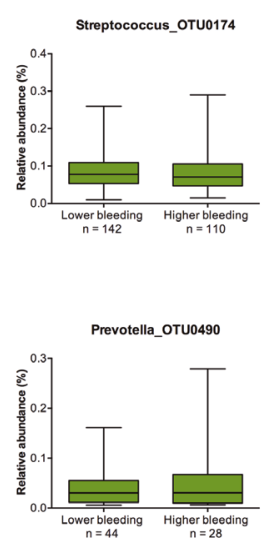

Unstimulated saliva
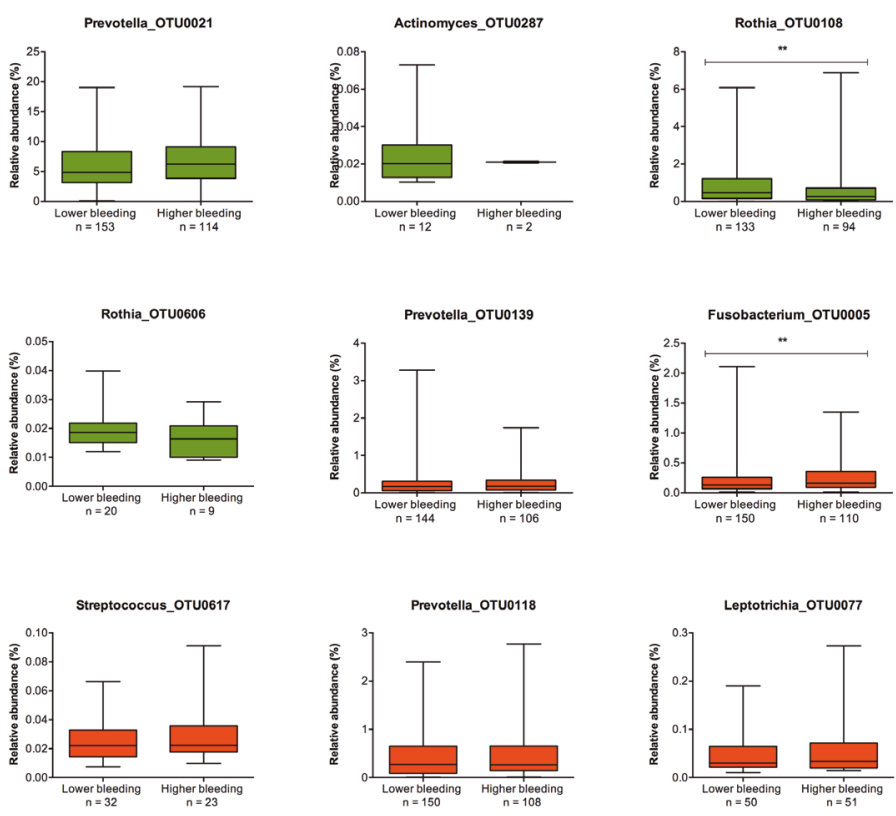

Plaque supragingival
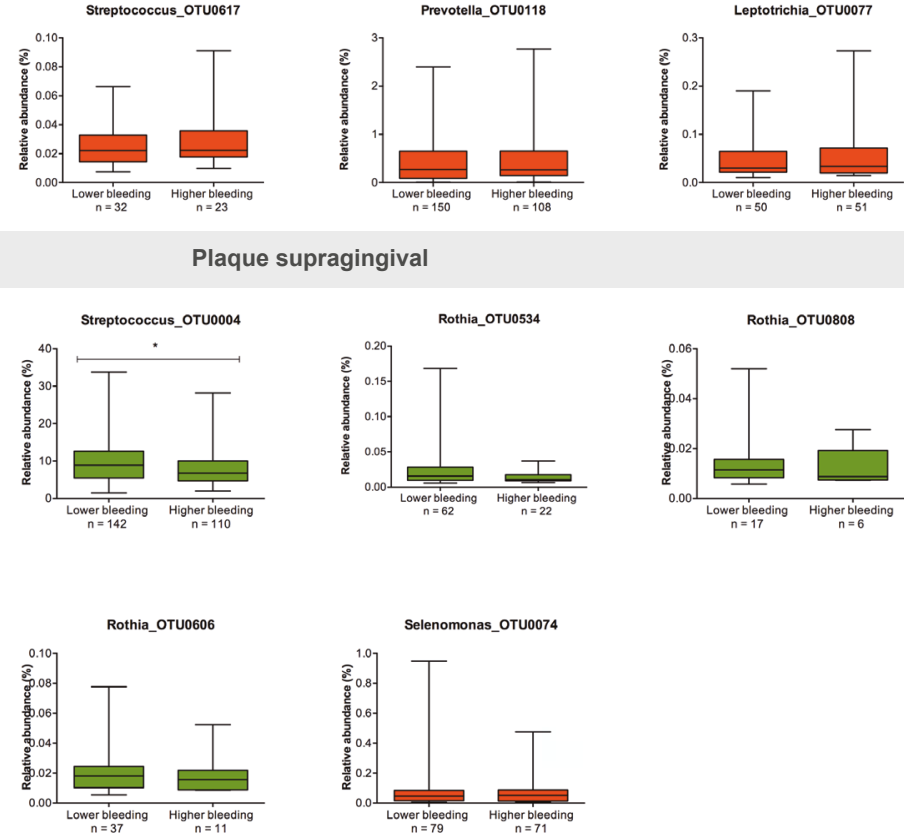

Figure S3. Relative abundance and prevalence of the identified OTUs in different oral cavity niches. (a) Relative abundance of OTUs in unstimulated saliva samples, which were found to be associated with gingival bleeding in supragingival plaque by elastic net regression. (b) Relative abundance of OTUs in supragingival plaque, which were found to be associated with gingival bleeding in unstimulated saliva by elastic net regression. Significant differences between groups were assessed by a two-tailed Mann-Whitney $U$ test $\left({ }^{*}, P<0.05 ;{ }^{* *}, P<0.01\right)$. Green and red boxplots represent OTUs that elastic net analysis identified to be negatively- and positively-associated with gingival bleeding. 
a.

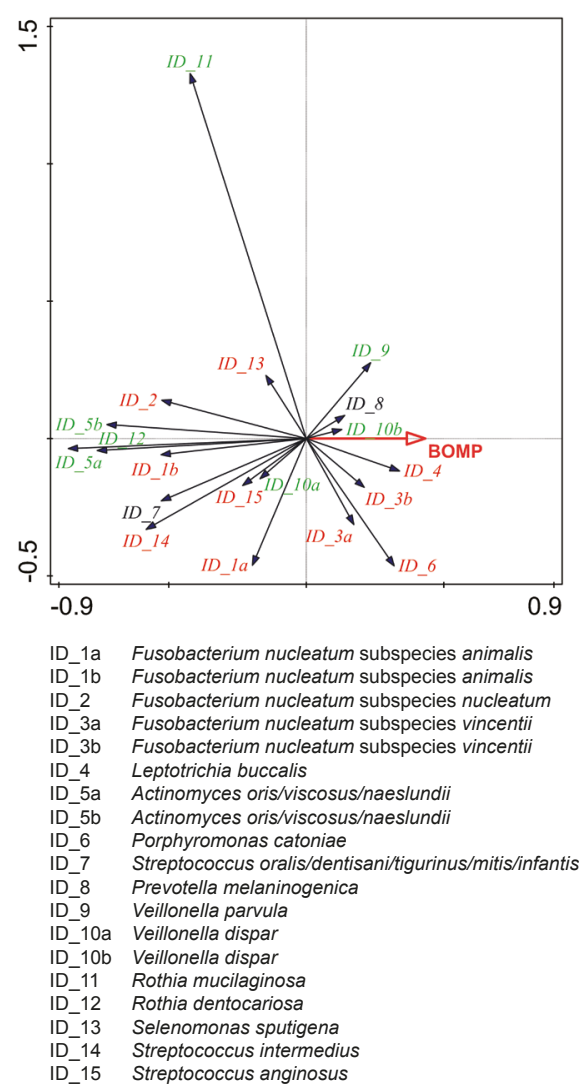

b.

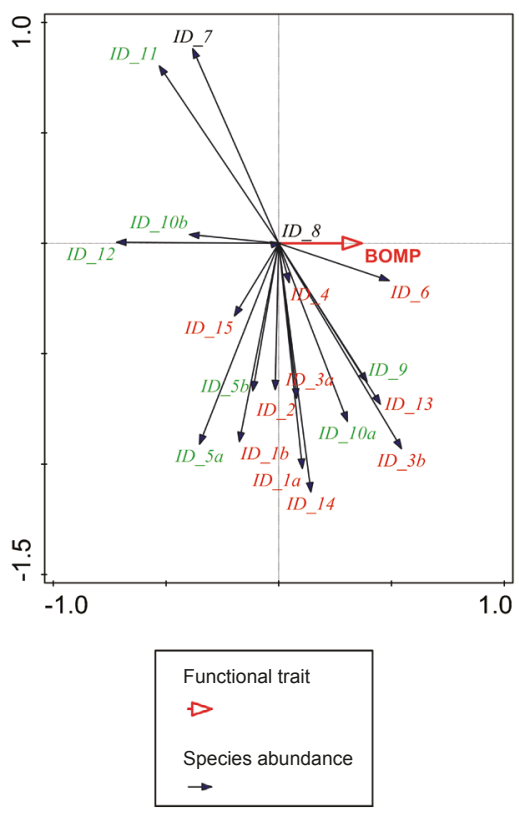

Figure S4. Relationship of the selected species and species-clusters with gingival bleeding during an experimental gingivitis challenge. (a) Relative abundance of the selected species in unstimulated saliva explained by the bleeding on marginal bleeding (BOMP) at the beginning of the challenge $(P=0.124)$. (b) Relative abundance of the selected species in supragingival plaque explained by the bleeding on marginal bleeding (BOMP) at the beginning of the challenge $(P=0.708)$. Species found to be negatively associated with gingival bleeding scores in the cross-sectional study by elastic net regression are represented with green labels, whereas positive associations are presented with red labels. Black labels represent bacterial species that were both positive and negative associated with gingival bleeding. 
Streptococcus anginosus (ID_15)

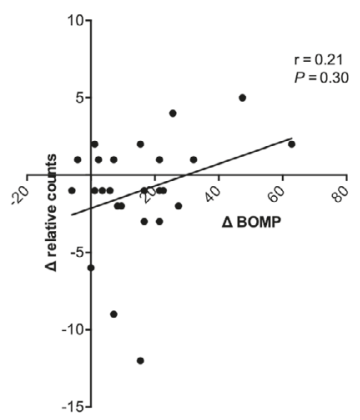

F. nucleatum subsp. nucleatum (ID_2)

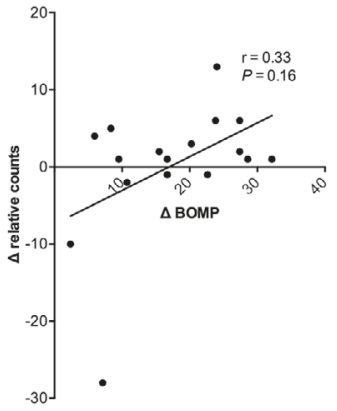

Rothia mucilaginosa (ID_11)

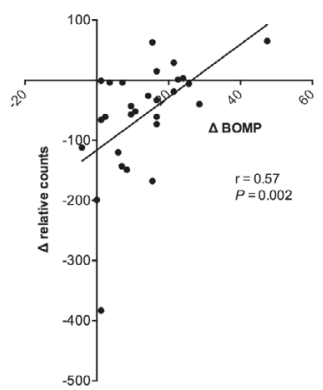

A. oris/viscosus/naes/undii (ID_5a)

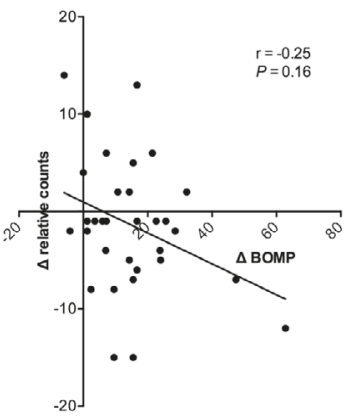

Veillonella parvula (ID_9)

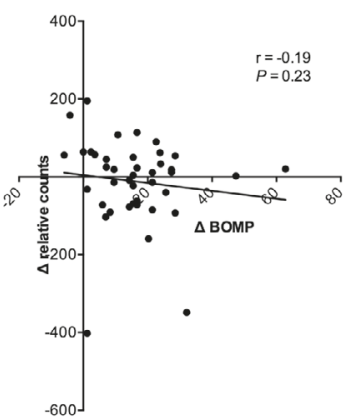

Figure S5. Spearman correlation analyses. The difference in relative abundance ( $\triangle$ relative counts) of the selected bacteria between the beginning (Day 0) and the peak (Day 14) of an experimental gingivitis challenge was correlated with the change in gingival bleeding ( $\triangle \mathrm{BOMP} \%$ ). 
Table S1. Selection of OTUs related to gingival bleeding by elastic net regression with stability selection. Operational taxonomic units (OTUs) associated with percentage of gingival bleeding, indicated by the positive or negative values of the fractional weight (weight standard deviation/ average weight).

\begin{tabular}{|c|c|c|c|c|}
\hline OTU_ID & Stability & Fractional weight & Genus & Niche \\
\hline 0tu0265 & 0.93 & 0.60 & Kingella & $\mathrm{PL}$ \\
\hline Otu0552 & 0.9 & 0.60 & Capnocytophaga & $\mathrm{PL}$ \\
\hline Otu0195 & 0.91 & 0.59 & Streptococcus & $\mathrm{PI}$ \\
\hline Otu1057 & 0.88 & 0.59 & Filifactor & $\mathrm{PL}$ \\
\hline Otu0073 & 0.91 & 0.59 & Rothia & US \\
\hline Otu0100 & 0.94 & 0.59 & Selenomonas & TC1 \\
\hline 0 tu0408 & 0.93 & 0.59 & Prevotella & US \\
\hline Otu0274 & 0.89 & 0.58 & Peptostreptococcaceae incertae sedis & US \\
\hline Otu0102 & 0.92 & 0.58 & Tannerella & TC1 \\
\hline Otu0018 & 0.91 & 0.58 & Fusobacterium & TC1 \\
\hline Otu0032 & 0.91 & 0.58 & Prevotella & US \\
\hline Otu1843 & 0.95 & 0.58 & Propionivibrio & $P L$ \\
\hline Otu1093 & 0.98 & 0.58 & Porphyromonas & $\mathrm{PL}$ \\
\hline Otu0077 & 0.92 & 0.57 & Leptotrichia & $\mathrm{PI}$ \\
\hline Otu0150 & 0.91 & 0.57 & Porphyromonas & $\mathrm{Pl}$ \\
\hline Otu1006 & 0.93 & 0.57 & Capnocytophaga & $\mathrm{PL}$ \\
\hline Otu0462 & 0.88 & 0.57 & Leptotrichia & $\mathrm{PL}$ \\
\hline Otu0306 & 0.92 & 0.57 & Actinobacillus & $\mathrm{PL}$ \\
\hline Otu0424 & 0.85 & 0.56 & Leptotrichia & $\mathrm{PI}$ \\
\hline Otu0195 & 1 & 0.56 & Streptococcus & US \\
\hline 0 tu0205 & 0.87 & 0.56 & Leptotrichia & $\mathrm{PL}$ \\
\hline Otu0052 & 0.84 & 0.56 & Dialister & US \\
\hline Otu0547 & 0.92 & 0.56 & Leptotrichia & $\mathrm{PL}$ \\
\hline Otu0005 & 0.89 & 0.56 & Fusobacterium & US \\
\hline Otu0556 & 0.9 & 0.56 & Treponema & $\mathrm{PL}$ \\
\hline Otu0243 & 0.99 & 0.56 & Eubacterium & US \\
\hline Otu0082 & 0.94 & 0.55 & Neisseria & US \\
\hline Otu0379 & 0.94 & 0.55 & Leptotrichia & $\mathrm{PL}$ \\
\hline Otu0286 & 0.9 & 0.55 & Fusobacterium & $\mathrm{PL}$ \\
\hline Otu0710 & 0.96 & 0.55 & Leptotrichia & $\mathrm{PL}$ \\
\hline Otu0081 & 0.97 & 0.55 & Prevotella & US \\
\hline Otu0031 & 0.97 & 0.54 & Prevotella & $\mathrm{PL}$ \\
\hline Otu0674 & 0.96 & 0.54 & Cardiobacterium & $\mathrm{PL}$ \\
\hline Otu0290 & 0.88 & 0.54 & Actinomyces & US \\
\hline Otu0131 & 0.91 & 0.54 & Streptococcus & $\mathrm{PL}$ \\
\hline Otu0183 & 0.97 & 0.53 & Capnocytophaga & $\mathrm{PL}$ \\
\hline Otu0297 & 0.96 & 0.53 & Fusobacterium & $\mathrm{PL}$ \\
\hline Otu1044 & 0.93 & 0.53 & Capnocytophaga & $\mathrm{PL}$ \\
\hline Otu0243 & 0.92 & 0.53 & Eubacterium & $\mathrm{PL}$ \\
\hline 0tu2730 & 0.89 & 0.52 & Streptobacillus & US \\
\hline Otu0306 & 0.89 & 0.52 & Actinobacillus & US \\
\hline Otu0994 & 0.92 & 0.52 & Actinomyces & US \\
\hline Otu0479 & 0.93 & 0.52 & Actinomyces & US \\
\hline Otu0172 & 0.91 & 0.52 & Leptotrichia & US \\
\hline Otu0058 & 0.89 & 0.51 & Prevotella & $\mathrm{PL}$ \\
\hline Otu0217 & 0.96 & 0.51 & Leptotrichia & US \\
\hline Otu0185 & 0.99 & 0.51 & Leptotrichia & $\mathrm{PL}$ \\
\hline Otu0185 & 1 & 0.51 & Leptotrichia & TC1 \\
\hline Otu0262 & 0.88 & 0.51 & Tannerella & $\mathrm{PL}$ \\
\hline Otu0427 & 0.92 & 0.51 & Neisseria & $\mathrm{PI}$ \\
\hline Otu0943 & 0.97 & 0.51 & Capnocytophaga & $\mathrm{PL}$ \\
\hline
\end{tabular}




\begin{tabular}{|c|c|c|c|c|}
\hline OTU_ID & Stability & Fractional weight & Genus & Niche \\
\hline Otu0195 & 0.96 & 0.51 & Streptococcus & $\mathrm{PL}$ \\
\hline Otu1059 & 0.97 & 0.50 & Treponema & US \\
\hline Otu1539 & 0.92 & 0.50 & Leptotrichia & PL \\
\hline Otu0330 & 0.93 & 0.50 & Streptococcus & US \\
\hline Otu0238 & 0.96 & 0.50 & Parvimonas & US \\
\hline Otu0012 & 0.96 & 0.50 & Campylobacter & PL \\
\hline Otu0192 & 0.93 & 0.49 & Catonella & PL \\
\hline Otu0246 & 0.95 & 0.48 & Fusobacterium & PL \\
\hline Otu1047 & 0.94 & 0.48 & Simonsiella & US \\
\hline Otu0258 & 0.92 & 0.47 & Fusobacterium & US \\
\hline Otu0125 & 0.95 & 0.47 & Capnocytophaga & US \\
\hline Otu0277 & 0.97 & 0.47 & Streptococcus & US \\
\hline Otu0164 & 0.98 & 0.47 & Prevotella & US \\
\hline Otu0362 & 0.95 & 0.45 & Actinomyces & $\mathrm{PL}$ \\
\hline Otu0823 & 0.94 & 0.45 & Porphyromonas & $\mathrm{PL}$ \\
\hline Otu0223 & 0.98 & 0.45 & Peptococcus & $\mathrm{PL}$ \\
\hline Otu0077 & 0.94 & 0.45 & Leptotrichia & $\mathrm{PL}$ \\
\hline Otu0118 & 0.93 & 0.44 & Prevotella & PL \\
\hline Otu0512 & 0.97 & 0.44 & Leptotrichia & $\mathrm{PL}$ \\
\hline Otu0617 & 0.96 & 0.43 & Streptococcus & $P L$ \\
\hline Otu0074 & 1 & 0.43 & Selenomonas & US \\
\hline Otu0006 & 0.97 & 0.43 & Fusobacterium & $\mathrm{PL}$ \\
\hline 0 tu0223 & 0.95 & 0.42 & Peptococcus & US \\
\hline Otu0311 & 0.99 & 0.41 & Fusobacterium & PL \\
\hline Otu0274 & 0.98 & 0.41 & Peptostreptococcaceae_incertae_sedis & PL \\
\hline Otu0237 & 0.99 & 0.40 & Johnsonella & $\mathrm{PL}$ \\
\hline Otu1021 & 0.99 & 0.38 & Porphyromonas & $\mathrm{PL}$ \\
\hline Otu0256 & 1 & 0.38 & Treponema & $\mathrm{PL}$ \\
\hline Otu0005 & 0.97 & 0.37 & Fusobacterium & PL \\
\hline Otu0139 & 0.97 & 0.35 & Prevotella & PL \\
\hline Otu0108 & 1 & -0.33 & Rothia & $\mathrm{PL}$ \\
\hline Otu0219 & 0.98 & -0.44 & Actinobacillus & US \\
\hline Otu0287 & 0.95 & -0.46 & Actinomyces & PL \\
\hline Otu0808 & 1 & -0.48 & Rothia & US \\
\hline Otu0021 & 0.97 & -0.49 & Prevotella & PL \\
\hline 0 tu0606 & 0.93 & -0.50 & Rothia & $\mathrm{PI}$ \\
\hline Otu0534 & 0.94 & -0.50 & Rothia & US \\
\hline Otu0004 & 0.91 & -0.50 & Streptococcus & US \\
\hline Otu0490 & 0.99 & -0.51 & Prevotella & PI \\
\hline Otu0054 & 0.92 & -0.52 & Corynebacterium & PL \\
\hline Otu0007 & 0.99 & -0.52 & Actinomyces & PL \\
\hline Otu0300 & 0.95 & -0.53 & Prevotella & PL \\
\hline Otu0174 & 0.88 & -0.54 & Streptococcus & US \\
\hline Otu0376 & 0.92 & -0.54 & Actinomyces & PL \\
\hline Otu0532 & 0.93 & -0.54 & Veillonella & US \\
\hline Otu0534 & 0.97 & -0.54 & Rothia & $\mathrm{PL}$ \\
\hline Otu0317 & 1 & -0.55 & Streptococcus & PL \\
\hline Otu0554 & 0.88 & -0.55 & Leptotrichia & US \\
\hline 0 tu0068 & 0.88 & -0.55 & Cupriavidus & $\mathrm{PL}$ \\
\hline Otu0367 & 0.9 & -0.55 & Actinomyces & $\mathrm{PL}$ \\
\hline Otu0048 & 0.87 & -0.55 & Streptococcus & US \\
\hline Otu0950 & 0.91 & -0.56 & Prevotella & US \\
\hline Otu0160 & 0.96 & -0.56 & Actinomyces & $\mathrm{PL}$ \\
\hline Otu0054 & 0.94 & -0.56 & Corynebacterium & $\mathrm{PI}$ \\
\hline Otu0504 & 0.87 & -0.56 & Neisseria & US \\
\hline
\end{tabular}




\begin{tabular}{|c|c|c|c|c|}
\hline OTU_ID & Stability & Fractional weight & Genus & Niche \\
\hline 0tu0417 & 0.91 & -0.57 & Actinomyces & $\mathrm{PI}$ \\
\hline 0 tu0233 & 0.89 & -0.57 & Veillonella & $\mathrm{Pl}$ \\
\hline Otu0053 & 0.89 & -0.57 & Prevotella & US \\
\hline Otu0585 & 0.93 & -0.57 & Corynebacterium & $\mathrm{PI}$ \\
\hline Otu0263 & 0.97 & -0.57 & Leptotrichia & $\mathrm{PI}$ \\
\hline Otu0007 & 0.92 & -0.57 & Actinomyces & TC1 \\
\hline Otu0351 & 0.86 & -0.57 & Rothia & $\mathrm{PI}$ \\
\hline Otu0849 & 0.91 & -0.57 & Rothia & US \\
\hline Otu1471 & 0.94 & -0.59 & Paraprevotella & $\mathrm{PI}$ \\
\hline Otu0700 & 0.93 & -0.59 & TM7_genus incertae sedis & US \\
\hline Otu0096 & 0.93 & -0.59 & Streptococcus & TC1 \\
\hline Otu1005 & 0.97 & -0.60 & Leptotrichia & $\mathrm{PL}$ \\
\hline
\end{tabular}

PI: plaque interproximal, PL: plaque supragingival, TC1: tongue coating anterior, US: unstimulated saliva.

Table S2. OTU species names assignment. The OTU representative sequences corresponding to the Prevotella, Actinomyces and Streptococcus genera were BLASTN against the HOMD, Greengenes, and NCBI databases.

\begin{tabular}{|c|c|c|}
\hline OTU\# & Fractional weight & Species \\
\hline 0tu0408 & 0.59 & Prevotella intermedia \\
\hline Otu0032 & 0.58 & Prevotella oris \\
\hline Otu0081 & 0.55 & Prevotella oris \\
\hline 0tu0031 & 0.54 & Prevotella veroralis \\
\hline 0tu0058 & 0.51 & Prevotella melaninogenica \\
\hline Otu0164 & 0.47 & Prevotella pleuritidis \\
\hline Otu0118 & 0.44 & Prevotella melaninogenica \\
\hline Otu0139 & 0.35 & Prevotella melaninogenica \\
\hline Otu0021 & -0.49 & Prevotella melaninogenica \\
\hline Otu0490 & -0.51 & Prevotella oulorum \\
\hline Otu0300 & -0.53 & Prevotella oulorum \\
\hline Otu0950 & -0.56 & Prevotella melaninogenica \\
\hline 0tu0053 & -0.57 & Prevotella melaninogenica/scopos \\
\hline Otu0290 & 0.54 & Actinomyces odontolyticus \\
\hline Otu0994 & 0.52 & Actinomyces odontolyticus/lingnae \\
\hline Otu0479 & 0.52 & Actinomyces odontolyticus/meyeri \\
\hline 0tu0362 & 0.45 & Actinomyces dentalis/orihominis \\
\hline 0 tu0287 & -0.46 & Actinomyces oris/viscosus/naeslundii \\
\hline Otu0007 & -0.52 & Actinomyces oris/viscosus/naeslundii \\
\hline Otu0376 & -0.54 & Actinomyces oris/viscosus/naeslundii \\
\hline 0tu0367 & -0.55 & Actinomyces massiliensis \\
\hline Otu0160 & -0.56 & Actinomyces georgiae \\
\hline Otu0417 & -0.57 & Actinomyces oris/naeslundii/johnsonii \\
\hline 0 tu0007 & -0.57 & Actinomyces oris/naeslundii/johnsonii \\
\hline Otu0195 & 0.59 & Streptococcus anginosus \\
\hline Otu0195 & 0.56 & Streptococcus anginosus \\
\hline Otu0131 & 0.54 & Streptococcus cristatus/oligofermentans/sinensis \\
\hline Otu0195 & 0.51 & Streptococcus anginosus \\
\hline 0 tu0330 & 0.50 & Streptococcus oralis/dentisani/tigurinus/mitis/infantis \\
\hline Otu0277 & 0.47 & Streptococcus vestibularis/salivarius \\
\hline 0tu0617 & 0.43 & Streptococcus intermedius/anginosus \\
\hline 0tu0004 & -0.50 & Streptococcus oralis/dentisani/tigurinus/mitis/infantis \\
\hline Otu0174 & -0.54 & Streptococcus oralis/dentisani/tigurinus/mitis/infantis \\
\hline Otu0317 & -0.55 & Streptococcus oralis/dentisani/tigurinus/mitis/infantis \\
\hline Otu0048 & -0.55 & Streptococcus sanguinis \\
\hline 0tu0096 & -0.59 & Streptococcus mutans \\
\hline
\end{tabular}




\section{Chapter}

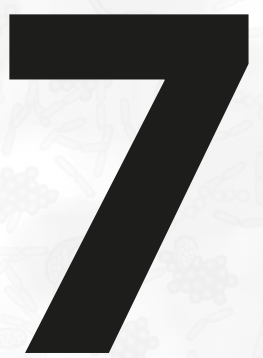

\section{Differential Effects of Oral Bacteria on Gingival Cells Revealed by Cell-Based Assays and Transcriptome Analysis}

Marcela M. Fernandez-Gutierrez ${ }^{1,2}$,

Peter van Baarlen², Lisa Robbers²,

Franka Siewers ${ }^{2}$,

Michiel Kleerebezem ${ }^{1,2^{*}}$ 
Oral epithelial integrity is essential for maintenance of barrier function and oral homeostasis. However, wounds can occur from daily activities (e.g. chewing and eating) and from the presence of inflammatory disorders such as periodontal diseases. Commensal oral bacteria can influence the process of gingival re-epithelialization. In a previous study, we showed that a group of bacteria negatively associated with gingival bleeding scores, a parameter used to assess the inflammatory state of the periodontal tissues, significantly increased re-epithelialization kinetics in vitro in comparison with a second group of oral bacterial that were positively associated with gingival bleeding scores. In the present study, we explore the modulatory mechanisms exerted by Actinomyces oris, Actinomyces viscosus, Veillonella parvula (negatively associated with gingival bleeding), and F. nucleatum subsp. animalis (positively associated with gingival bleeding) on gingival re-epithelialization using cell-based assays in combination with transcriptomics. Our results show that in comparison with scratched cell monolayers left untreated, exposure to $F$. nucleatum subsp. animalis was characterized by a strong induction of pro-inflammatory cytokines and activation of regulatory genes involved in cell proliferation and apoptosis. In contrast, treatment with the Actinomyces spp. and V. parvula led to downregulation of genes participating in the $p 53$ signalling pathway, including the 'stress sensor' GADD45A, resulting in increased cell viability in vitro. Additionally, we propose that $A$. oris and $V$. parvula may secrete soluble compounds that act as chemoattractants of gingival cells, which could partially explain the stimulatory effect observed with these bacteria on cell migration. This study contributes to our understanding of the mechanisms by which oral commensal bacteria can influence re-epithelialization and woundrepair, and thereby play pivotal roles in the maintenance of oral health or the progression towards a disease state. 


\section{Introduction}

The oral cavity is the second most diverse microbial community in the human body, harbouring over 700 microbial species that have adapted to colonize and grow within specific niches of the oral cavity ${ }^{36}$. Whereas some species, such as Porphyromonas gingivalis, have been extensively studied for their involvement in the development of disease ${ }^{234}$, most microbes live in a stable and symbiotic relationship with the host and play an important role in the maintenance of oral health ${ }^{33}$. However, functionality of the oral commensals has only recently started to be investigated and current advances in technology such as next-generation sequencing ${ }^{235}$, have started to unravel the complexity of the oral microbiome and its interplay with health and disease ${ }^{66,226}$.

Microbiota profiling studies have shown that members belonging to the genera Streptococcus, Fusobacterium, Veillonella and Actinomyces are common residents of a healthy oral cavity ${ }^{44}$. We recently performed a cross-sectional study of a healthy cohort $(n=268)$ in which we determined the oral microbiota of the participants by 165 rRNA sequencing ${ }^{211}$ and recorded the gingival bleeding scores for each individual ${ }^{177}$. The gingival bleeding scores are used as a clinical parameter to characterize the degree of gingival inflammation and therefore, to assess the risk for developing periodontal diseases ${ }^{6}$. The operational taxonomic units (OTUs) assigned to the species groups Actinomyces oris/viscosus/naes/undii and Veillonella atypica/dispar/ parvula were negatively associated with gingival bleeding, suggesting that these microorganisms may have a beneficial role in oral health. We explored this notion further in vitro and found that from the panel of oral commensal bacteria ( $n=19$ ) tested, A. oris, A. viscosus and V. parvula were the strongest and most consistent stimulators of gingival reepithelialization ${ }^{236}$, which is crucial for maintenance of oral epithelial integrity and barrier function ${ }^{7}$. In contrast, we showed that Fusobacterium nucleatum subsp. animalis was positively associated with gingival bleeding in vivo and had a dose-dependent effect on re-epithelialization ${ }^{236}$. Exposure of gingival cells to $F$. nucleatum subsp. animalis at a multiplicity of infection (MOI) of 100 resulted in enhanced re-epithelialization kinetics, whereas a higher dosage (MOI 500) had an inhibitory effect ${ }^{236}$.

In the present study, we aimed to investigate the modulatory mechanisms exerted by the bacterial panel consisting of A. oris, A. viscosus, V. parvula and $F$. nucleatum subsp. animalis on gingival re-epithelialization using cell-based assays in combination with an un-biased transcriptome approach. In comparison with the cells that were left untreated during reepithelialization, treatment with the Actinomyces spp. and V. parvula had a modest impact on the transcriptome and led to the downregulation of central regulators of cell death (senescence) and stress-associated genes, which was in line with the increased cell viability observed in vitro. In contrast, stimulation of gingival cells with $F$. nucleatum subsp. animalis led to a strong upregulation of genes involved in proliferation, inflammatory responses, and apoptosis. Finally, we show that the stimulatory effects of $A$. viscosus and $F$. nucleatum subsp. animalis on epithelial cell migration require direct bacterialhost cell contact, whereas A. oris and $V$. parvula are proposed to stimulate migration by secreted soluble compounds that act as chemoattractants for gingival cells. 


\section{Materials and Methods}

\section{Cell line and bacterial strains}

The gingival cell line Ca9-22 (JCRB0625) was purchased from the National Institute of Biomedical Innovation JCRB Cell Bank (Osaka, Japan). The cells were grown in Dulbecco's Modified Eagle Medium (DMEM) Glutamax ${ }^{\mathrm{TM}}$ (Gibco, Invitrogen, Paisley, UK) supplemented with $10 \%$ fetal calf serum (FCS), $100 \mathrm{U} / \mathrm{mL}$ penicillin and $100 \mu \mathrm{g} / \mathrm{mL}$ streptomycin (SigmaAldrich, MO, USA). The cell line was incubated at $37^{\circ} \mathrm{C}$ in a humidified atmosphere containing $5 \% \mathrm{CO}_{2}$ and was passaged every three days. Veillonella parvula HSIVP1 was kindly provided by Dr. Tom van den Bogert from the Laboratory of Microbiology of the Wageningen University (Wageningen, The Netherlands). Actinomyces oris DSM-23056, Actinomyces viscosus DSM-43798 and Fusobacterium nucleatum subspecies animalis DSM-19679 were purchased from the German Collection of Microorganisms and Cell Cultures (DSMZ). V. parvula and F. nucleatum were grown in Veillonella medium (DSMZ medium nr. 136) and PYG medium (DSMZ medium nr. 104), respectively. A. oris was grown in PYG medium supplemented with $0.4 \%$ glucose, $0.1 \%$ cellobiose, $0.1 \%$ maltose and $0.1 \%$ soluble starch. V. parvula, F. nucleatum, and A. oris were grown anaerobically at $37^{\circ} \mathrm{C}$ using the $\mathrm{GaSPak}^{\mathrm{TM}}$ EZ Anaerobe Container System Sachets (Becton, Dickinson and Company, NJ, USA). A. viscosus was grown at $37^{\circ} \mathrm{C}$ in a humidified incubator with $5 \% \mathrm{CO}_{2}$ in Brain Heart Infusion (BHI) broth (BD Difco, Le Pont de Claix, FR) supplemented with casein (10 g/L), glucose $(5.0 \mathrm{~g} / \mathrm{L})$ and yeast extract $(5.0 \mathrm{~g} / \mathrm{L})$.

\section{Bacterial preparation}

The strains were grown until early stationary phase, after which glycerol stocks ( $15 \% \mathrm{v} / \mathrm{v})$ were prepared and stored at $-80^{\circ} \mathrm{C}$ until further use. To perform the cell-based assays, the glycerol stocks were thawed and the bacteria were spun down by centrifugation (4,000 x g, 7 minutes at room temperature). The spent media were removed and the bacterial pellets were re-suspended in FCS- free DMEM without antibiotics to obtain the original optical density at $600 \mathrm{~nm}(0 D 600)$. The samples were further diluted to achieve a multiplicity of infection (MOI) of 100 and 500 using a generalized conversion rate of $0 D 600$ of 1 corresponding to approximately $5 \times 10^{8} \mathrm{CFU} / \mathrm{ml}$.

\section{Scratch assay and collection of samples for RNA isolation}

The scratch assay was carried out as previously described ${ }^{155}$. Briefly, Ca9-22 cells were seeded in 96-well plates at a density of $3.5 \times 10^{4}$ cells/well and incubated at $37^{\circ} \mathrm{C}$ until a confluent monolayer was formed. The cells were starved in FCS-free DMEM for 2 hours and fluorescently labelled with $2 \mu \mathrm{g} / \mathrm{ml}$ Hoechst 33342 (Molecular Probes, OR, USA) and $2 \mu \mathrm{M}$ CellTracker ${ }^{\mathrm{TM}}$ Red CMTPX (Molecular Probes), after which the monolayers were scratched using the HTS Scratcher (Peira, Antwerpen, BE). After washing the cells twice with PBS, the bacterial treatments were added at an MOI of 100 and incubated at $37^{\circ} \mathrm{C}$ with $5 \% \mathrm{CO}_{2}$. FCS-free DMEM was used as untreated control and $4 \mathrm{ng} / \mathrm{ml}$ human transforming growth factor a (hTGFa) served as positive control for the induction of cell migration and proliferation ${ }^{162,237}$. Re-epithelialization was followed by acquiring images every 20 minutes for a period of 5 hours using the BD Pathway 855 Bioimaging System (BD Biosciences, CA, USA). The modified Gompertz function ${ }^{105}$ was used to model the number of cells infiltrating into the scratched area over time in order to extract two biologically relevant parameters that describe the re-epithelialization kinetics: the $\mu_{\mathrm{m}}$ (repair rate, cells minute ${ }^{-1}$ ) and the $A$ (maximum number of cells in the scratched area) parameters. In parallel to the image-based scratch assay, an identical experiment was carried out using unlabelled cells for transcriptome 
analysis. Three technical replicates were collected directly after performing the scratch in the cell monolayer $(\mathrm{t}=0 \mathrm{~min})$ and at 40, and 120 minutes after exposure to each of the preparations. For sample collection, cells were washed twice with PBS, lysed with $200 \mu$ l buffer RLT RNeasy Micro kit (Qiagen, Venlo, NL), and immediately stored at $-80^{\circ} \mathrm{C}$ until RNA isolations were performed.

\section{Migration assay}

To evaluate whether soluble bacterial products could act as chemoattractants for gingival cells, a migration assay using the Boyden chambers ${ }^{238}$ was performed. Cells were seeded at a density of $4.3 \times 10^{4}$ on transwell inserts (FluoroBlok ${ }^{\mathrm{TM}}$, Corning, NY, USA) with $8.0 \mu \mathrm{m}$ pore size. Upon chemoattraction, the epithelial cells pass through the pores of the membrane to the basolateral side of the transwell insert. Selected bacterial preparations (MOI 100 and 500) were added to the basolateral compartment after starving the cells for 2 hours in FCS-free DMEM. TGFa was used as a positive control at a concentration of $4 \mathrm{ng} / \mathrm{ml}$ and FCS-free DMEM was used as non-treated control. After 5 hours of incubation, the cells that migrated into the basolateral side of the insert were fixed using $4 \%$ formaldehyde (FA) and labelled with $2 \mu \mathrm{g} / \mathrm{ml}$ Hoechst 33342 (Molecular Probes, OR, USA). The inserts were then excised and mounted on microscope slides with Fluoromount ${ }^{\mathrm{TM}}$ aqueous mounting medium (Sigma-Aldrich). Fluorescent images from two different frames per insert $(n=3)$ were acquired with a 4X objective using a Leica DM6b upright microscope and $350 \mathrm{~nm}$ excitation filter. The number of cells migrating from the apical to the basolateral compartment was quantified using an automated pipeline developed in CellProfiler (version 2.1.1).

\section{Annexin V Live/Dead Staining}

To quantify the viability of gingival cells after exposure to the bacterial preparations, the eBiosiences ${ }^{\mathrm{TM}}$ Annexin V Apoptosis Detection Kit APC (Invitrogen, CA, USA) was used in combination with CountBright ${ }^{\mathrm{TM}}$ Absolute Counting Beads (Invitrogen) to determine numbers of viable and dead cells upon each treatment. Cells were seeded at a density of $2.6 \times 10^{5}$ cells per well in 24-well plates. After overnight incubation and 2 hours starvation in FCS-free DMEM, cells were scratched, washed once with PBS, and exposed to the bacterial preparations (MOI 100) for 5 hours. After incubation, cells were washed with PBS and detached from the well using trypsin. Detached cells were harvested by centrifugation (1,000 x g, 5 minutes at room temperature) and then washed once with PBS and once with 1 X Binding Buffer. After centrifugation, the cells were re-suspended in $1 X$ Binding Buffer and stained with Annexin V-APC ( $5 \mu \mathrm{l}$ in $100 \mu \mathrm{l}$ of cell suspension). Annexin V is a calcium-dependent phospholipid-binding protein that preferentially binds to phosphatidylserine (PS). Upon initiation of apoptosis, PS translocates to the extracellular membrane and can then be detected by fluorescently labelled extracellular annexin $\mathrm{V}^{239}$. Cells undergoing late apoptosis or necrosis were visualized by addition of $6 \mu \mathrm{l} /$ sample propidium iodide (PI). A combination of $100 \mathrm{ng} / \mathrm{ml}$ tumor necrosis factor a (TNFa; Invitrogen) and $100 \mathrm{ng} / \mathrm{ml}$ interferon $\gamma$ (IFNץ; Invitrogen) were used for cell death induction. To enumerate cells, counting beads were added to each sample ( $1.09 \times 10^{4}$ beads $/$ sample) prior to the flow cytometry measurements. A total of 10,000 events were measured and the cell counts were performed using the FCS express software (De Novo Software, CA, USA) Concentration Calculator. 


\section{IL-6 and IL-8 measurements}

To determine the concentration of interleukin-6 (IL-6) and interleukin-8 (IL-8) produced upon treatment with the selected bacteria (MOI 100), supernatants of the epithelial cell cultures $(n=3)$ were collected after 5 hours of incubation in the scratch assay. Human IL-6 and IL-8 measurements were performed with the Luminex MAGPIX System (Bio-Rad Laboratories, CA, USA) using the Bio-Plex Pro ${ }^{\text {TM }}$ Human Chemokine IL-6 and IL-8/CXCL8 (Bio-Rad) sets, respectively, according to the manufacturer instructions.

\section{RNA isolation and microarray processing}

Total RNA was isolated from the gingival cells using the RNeasy Micro kit (Qiagen, Venlo, NL) with an on-column DNase digestion step according to the manufacturer's instructions. RNA quality was assessed using RNA 6,000 nanochips on the Agilent 2100 Bioanalyzer (Agilent Technologies, Amsterdam, NL). Total RNA (100 ng) was labelled using an Affymetrix WT plus reagent kit and hybridized to whole genome Genechip Human Gene 2.1 ST arrays detecting 25,088 genes (Affymetrix, CA, USA). Sample labelling, hybridization to chips and image scanning was performed according manufacturer's instructions. Microarray analysis was performed using the MADMAX pipeline for statistical analysis of microarray data ${ }^{240}$. Various advanced quality metrics, diagnostic plots, and classification methods were applied to assure that only arrays that passed the most rigorous quality controls were used in the subsequent analyses ${ }^{241}$. For further analysis, a custom annotation was used based on reorganized oligonucleotide probes, which combines all individual probes for a gene ${ }^{242}$. Expression values were calculated using robust multichip average (RMA) method, which includes quantile normalization ${ }^{243}$. Differentially expressed probe sets were identified using linear models, applying moderated $\mathrm{t}$-statistics that implement empirical Bayes regularization of standard errors using Bioconductor's limma package ${ }^{244}$.

\section{Gene set enrichment analysis and biological interpretation of transcriptome datasets}

MultipleExperiment Viewer (MeV) (http://mev.tm4.org/\#/welcome) ${ }^{245}$ was used to perform a principal component analysis (PCA) using the log2 RMA expression values obtained from a scratched monolayer of cells exposed to the bacterial preparations, hTGFa or non-treated control at 40 and 120 minutes after performing a scratch in a confluent monolayer of cells. To identify enriched gene sets that result from cell damage and treatment with the different bacterial preparations, we performed gene set enrichment analyses (GSEA) using the pre-ranked GSEA tool developed by the Broad Institute (http://software.broadinstitute.org/gsea) ${ }^{246}$. Gene t-statistic values of each treatment at 120 minutes were ranked in relation to the moment of creation of the scratch $(t=0 \mathrm{~min})$, and the non-treated control at 120 min during re-epithelialization. The gene sets in the Kyoto Encyclopedia of Genes and Genomes (KEGG) served as database using a relatively tolerant false discovery rate (FDR) q-value of $<0.25$ as cut-off. To determine protein-protein interactions, the differentially expressed leading genes with a 'strict' FDR p-value $<0.05$ in the enriched gene sets were retrieved and visualized in Cytoscape ${ }^{247}$ using the Bisogenet plugin ${ }^{248}$. 


\section{Results}

\section{Re-epithelialization stimulating oral commensals distinctly impact on cell migration}

In a previous study ${ }^{236}$, we performed a screening to determine the influence of a panel of commensal oral bacteria on gingival re-epithelialization using an in vitro model ${ }^{155}$. The stimulatory effects formerly observed were confirmed with the subpanel of bacteria consisting of A. oris, A. viscosus. V. parvula and F. nucleatum subsp. animalis at an MOI 100. When compared to the non-treated control, consisting of scratched cell monolayers exposed to FCS-free DMEM, addition of the bacterial preparations into the scratched monolayer of cells resulted in a significant increase $(P<0.0001)$ in the repair rate $\left(\mu_{\mathrm{m}}\right.$ parameter) as well as in the number of cells infiltrating the scratched area (A parameter) during the 5 hours assay period (Fig. 1a).

To explore whether the increased re-epithelialization kinetics observed resulted from direct contact with the bacteria or if potential secreted products could act as chemoattractants for the gingival cells, we performed a migration assay using Boyden chambers ${ }^{238}$ at two different MOI (100 and 500). This assay consists on the quantification of cells migrating from the apical side of a porous transwell insert, towards a bacterial stimulus present in the basolateral compartment of the transwell system. At an MOI of 100 , the bacteria or their secreted products were not able to significantly stimulate migration of gingival cells through the insert (data not shown). However, addition of $A$. oris and $V$. parvula at an MOI of 500 significantly $(P<0.0001$ and $P=0.03$, respectively) increased migration of gingival cells towards the basolateral compartment that contained the bacteria (Fig. 1b). Conversely, the higher dosage of $A$. viscosus or F. nucleatum subsp. animalis did not elicit a similar migration-promoting effect. 


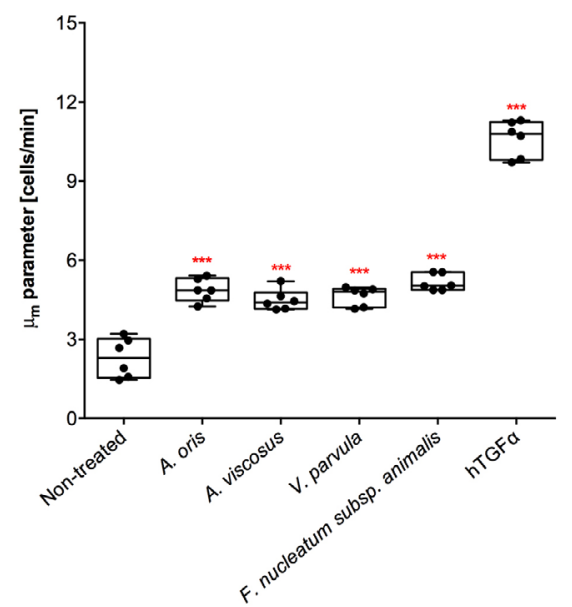

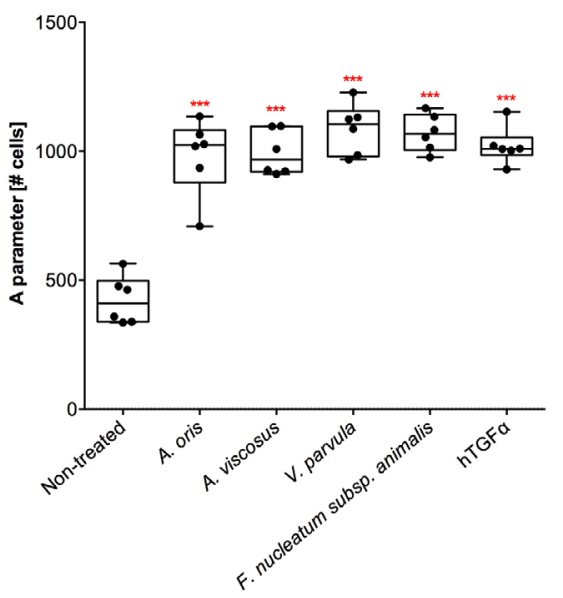

b.

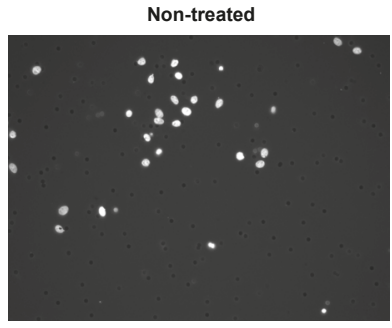

V. parvula

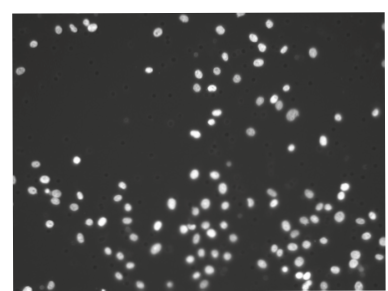

A oris

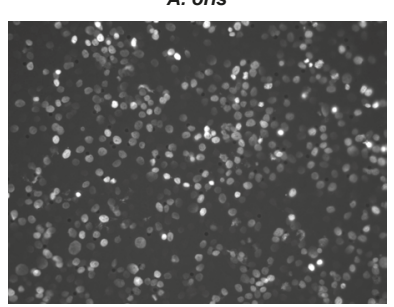

F. nucleatum subsp. animalis

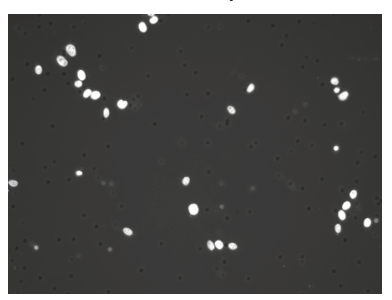

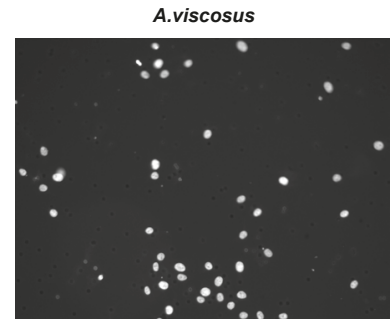

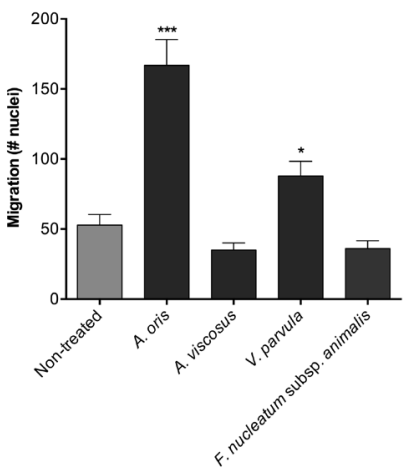

Figure 1. Effect of oral commensal bacteria on cell migration after $\mathbf{5}$ hours using different assays. (a) Different bacterial preparations (MOI 100) were added into scratched cell monolayers. Human transforming growth factor a (hTGFa; $4 \mathrm{ng} / \mathrm{ml}$ ) served as positive control for cell migration and proliferation. Re-epithelialization kinetics were determined by the $\mu_{\mathrm{m}}$ (repair rate) and A parameters (maximum number of cells inside the scratched area). (b) Gingival cells were seeded on the apical side of a porous transwell insert and the bacterial preparations ( $\mathrm{MOI} 500$ ) were added in the basolateral compartment. Nuclei were fluorescently labelled (Hoechst 33342; $2 \mu \mathrm{g} / \mathrm{ml}$ ) and images of the basolateral side of the inserts were acquired. Cells that migrated from the apical to the basolateral side upon chemotaxis were quantified using CellProfiler. Significant differences from the non-treated control were assessed by a one-way ANOVA using Dunnett's test for multiple comparisons ( $\left.{ }^{*}, P<0.05 ;{ }^{* * *}, P<0.0001 ; n=6\right)$. 


\section{Oral commensal bacteria distinctly impact epithelial inflammation and cell death}

To further investigate how these bacteria could influence the re-epithelialization kinetics of gingival cells, we determined the inflammatory response of the cells by measuring the amount of interleukin-6 (IL-6) and interleukin-8 (IL-8) produced by cells exposed to the bacterial preparations ( $\mathrm{MOI} 100)$ or the non-treated control during the re-epithelialization assay. After 5 hours of stimulation with $A$. oris, $A$. viscosus and $V$. parvula the secreted levels of both cytokines remained below the detection limits $(1.41 \mathrm{pg} / \mathrm{ml})$. In contrast, F. nucleatum subsp. animalis induced significant production of IL-6 $(P<0.0001)$ and IL-8 $(P=0.02)$ in comparison with the non-treated control, reaching levels that were only approximately 2 -fold lower than those observed with TNFa and IFNץ (100 ng/ml each) used as positive inducer of inflammatory responses (Fig. 2a).

a.

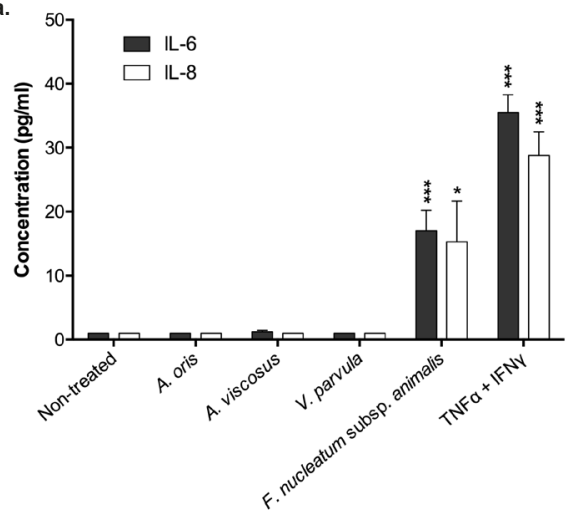

b.
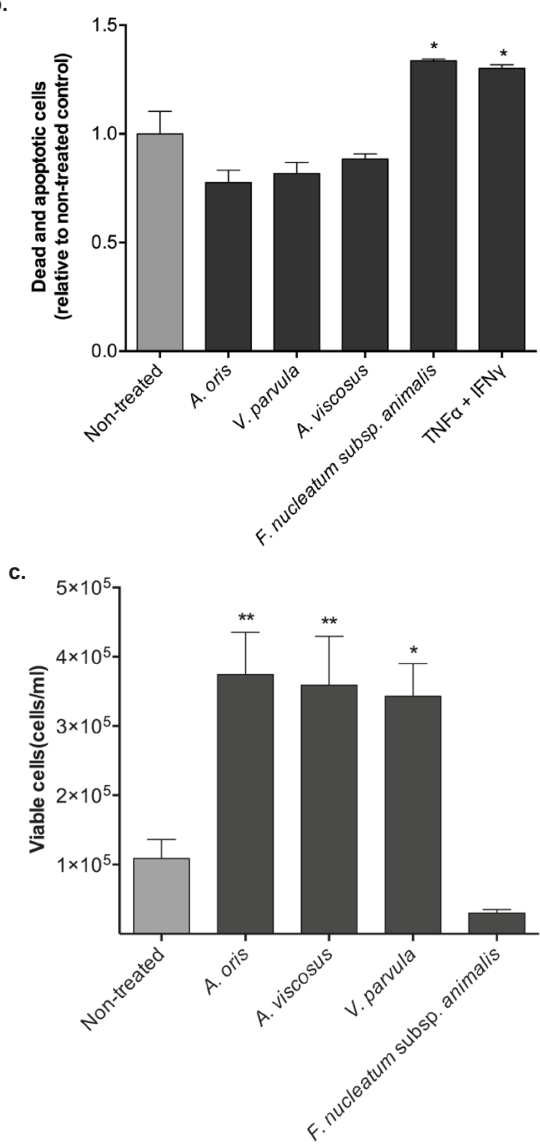

Figure 2. Inflammatory response, apoptosis and cell death determination after 5 hours exposure to the bacterial preparations. (a) Concentration of IL-6 and IL-8 in epithelial cell supernatants. A combination of human tumor necrosis factor a (TNFa) and interferon $\gamma$ (IFNY) served as positive control $(100 \mathrm{ng} / \mathrm{ml}$ each) to elicit pro-inflammatory cellular responses. (b) Enumeration of apoptotic and dead cells (Annexin V +, $\mathrm{Pl}+$ ) expressed relative to the non-treated control. (c) Concentration of viable cells (Annexin V -, PI -). Bacterial preparations were added at an MOI 100. Significant differences from the non-treated control were assessed by a one-way ANOVA using Dunnett's test for multiple comparisons $\left({ }^{*}, \mathrm{P}<0.05 ;{ }^{* *}\right.$, $\left.\mathrm{P}<0.01,{ }^{* * *}, \mathrm{P}<0.0001 ; \mathrm{n}=3\right)$. 


\section{8}

Inflammatory responses may impact re-epithelialization in various ways, by for instance, modulating cell death. To investigate this, the total number of apoptotic and dead cells was determined by enumeration of annexin $\mathrm{V}$ and propidium iodide (PI) positive cells after 5 hours exposure to each treatment in the reepithelialization assay. In comparison to the non-treated control, exposure of gingival cells to $F$. nucleatum subsp. animalis significantly increased $(P=0.02)$ the number of apoptotic and dead cells to a level that was comparable to the one observed with the TNFa and IFNy control (Fig. 2b). In addition, the enumeration of annexin $\mathrm{V}$ and PI negative cells revealed a significantly higher cell viability after treatment with $A$. oris $(P=0.005), A$. viscosus $(P=0.007)$ or $V$. parvula $(P=0.01)$, suggesting that these treatments might support cell survival and/or promote cell proliferation. Taken together, these findings exemplify the distinct physiological effects in gingival epithelial cells elicited by these bacteria, which could explain their differential impact on re-epithelialization kinetics in vitro.

\section{Transcriptional responses of gingival cells to epithelial-wounding and hTGFa treatment}

To further explore the modulatory effects of the commensal bacteria on gingival re-epithelialization kinetics in an unbiased, genome-wide manner, we conducted transcriptome analyses of the scratched monolayers of cells collected 40 and 120 minutes after exposure to each of the bacterial preparations and the controls. FCS-free DMEM served as nontreated control (baseline), whereas hTGFa acted as a positive control for cell proliferation and migration via activation of the epidermal growth factor receptor (EGFR) ${ }^{162,237}$. We first examined the baseline and cells treated with hTGFa at the two timepoints using principal component analysis (PCA). The PCA was constructed using as input robust multichip average (RMA)-normalised expression values from the complete microarray dataset (i.e. 25,088 genes). After 40 minutes, the transcriptomes corresponding to the controls clustered closer to each other in the PCA plot (Fig. 3). However, after 120 minutes of re-epithelialization, we could observe a clear difference between the transcriptomes, illustrated by a larger separation between the controls in the PCA plot (Fig. 3).

To explore the transcriptional responses resulting from epithelial-wounding, we conducted gene set enrichment analysis (GSEA) between the non-treated cells at 120 minutes and the moment in which the scratch was created ( $t=0 \mathrm{~min}$ ) using the Kyoto Encyclopedia of Genes and Genomes (KEGG) pathway database. Among the upregulated gene sets, we identified gene sets associated with intracellular signalling induced by cell-cell contact, including ECM receptor interaction, focal adhesion, mitogen-activated protein kinase (MAPK), ErbB, JAK-STAT, and TGF signalling pathways (Table S1). In contrast, the significantly downregulated gene sets were mainly related to basal metabolism and cell cycle (Table S2). Treatment with hTGFa led to further up- and downregulation of the same processes, which is in agreement with its impact in re-epithelialization (Table S3 and Table S4). 


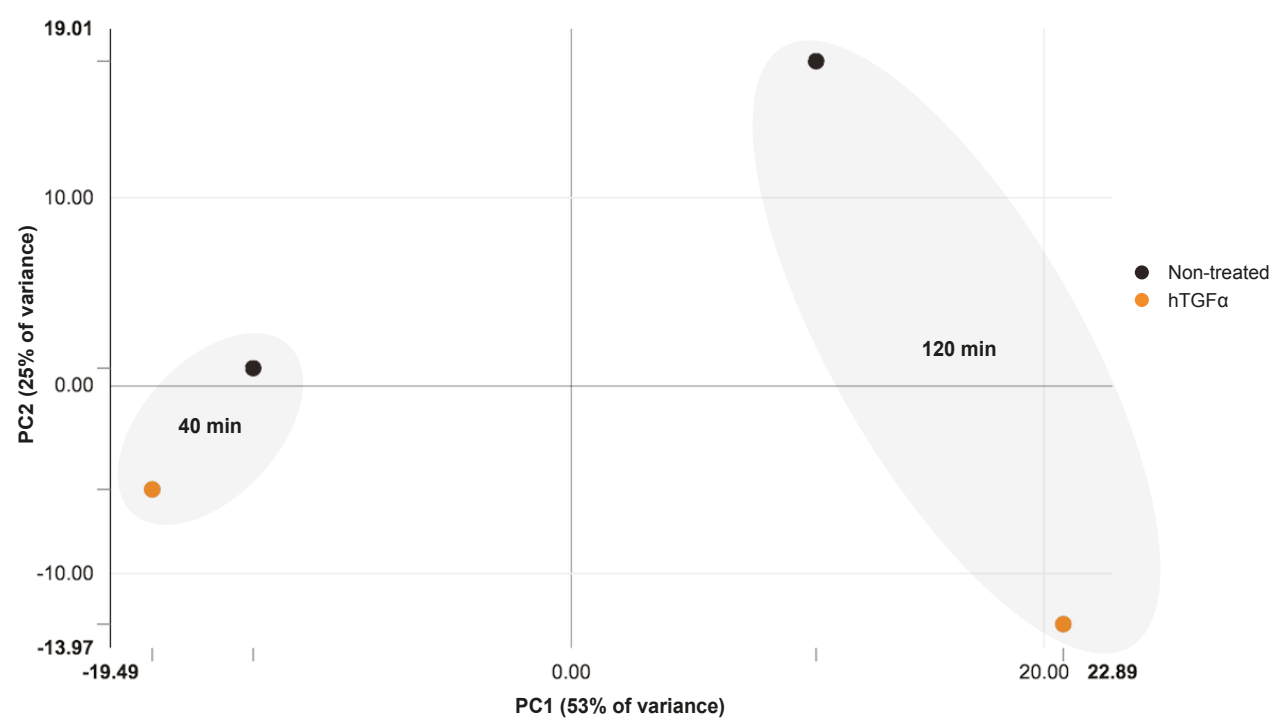

Figure 3. Principal component analysis of the transcriptomes of cells exposed to hTGFa and the non-treated control cells during reepithelialization. Input data consisted of the mean raw expression values $(n=3)$ representing the transcriptomes obtained from gingival cells exposed to hTGFa or the non-treated control at 40 and 120 minutes after introducing a scratch in a confluent monolayer of cells.

We were interested to identify potential regulators of the gene sets that we considered to be relevant to wound healing. To this end, differentially expressed leading genes from the enriched gene sets were used to construct protein-protein interaction networks for each comparison using the Bisogenet plugin ${ }^{248}$ in Cytoscape ${ }^{247}$ (Fig. S1 and Fig. S2). The network representations made it easier to visually identify potential regulators that could be further explored in Cytoscape. The responses of the gingival cells following the creation of the scratch were associated with three central regulatory nodes, namely epidermal growth factor receptor (EGFR), c-Myc (MYC), and JunD (JUND) (Fig 4 and Fig. S1). Furthermore, in comparison with the non-treated control, stimulation with hTGFa led to a significantly increased expression of c-MYC and C-JUN proto-oncogenes as well as further upregulation of several growth factors, including platelet-derived growth factor subunit B (PDGFB), vascular endothelial growth factors A (VEGFA), and C (VEGFC) (Fig. 4 and Table S5). Negative feedback regulators of EGFR signalling, including members of the sprouty/spread family of proteins (SPRY2, SPRY4, SPRED2) and dual-specificity phosphatases (DUSP1, DUSP5, DUSP6) were also upregulated after treatment with hTGFa (Fig. 4 and Table S5). In addition, hTGFa induced genes encoding pro-inflammatory cytokines and stress-associated genes, including chemokine (C-X-C motif) ligand 3 (CXCL3), interleukin 6 family cytokine (LIF), and interleukin 1 alpha (IL1A) as well as growth arrest and DNA damage inducible alpha protein (GADD45A), nuclear receptor subfamily 4 group A member 1 (NR4A1), and tumor necrosis receptor superfamily member 10A (TNFRSF10A). 


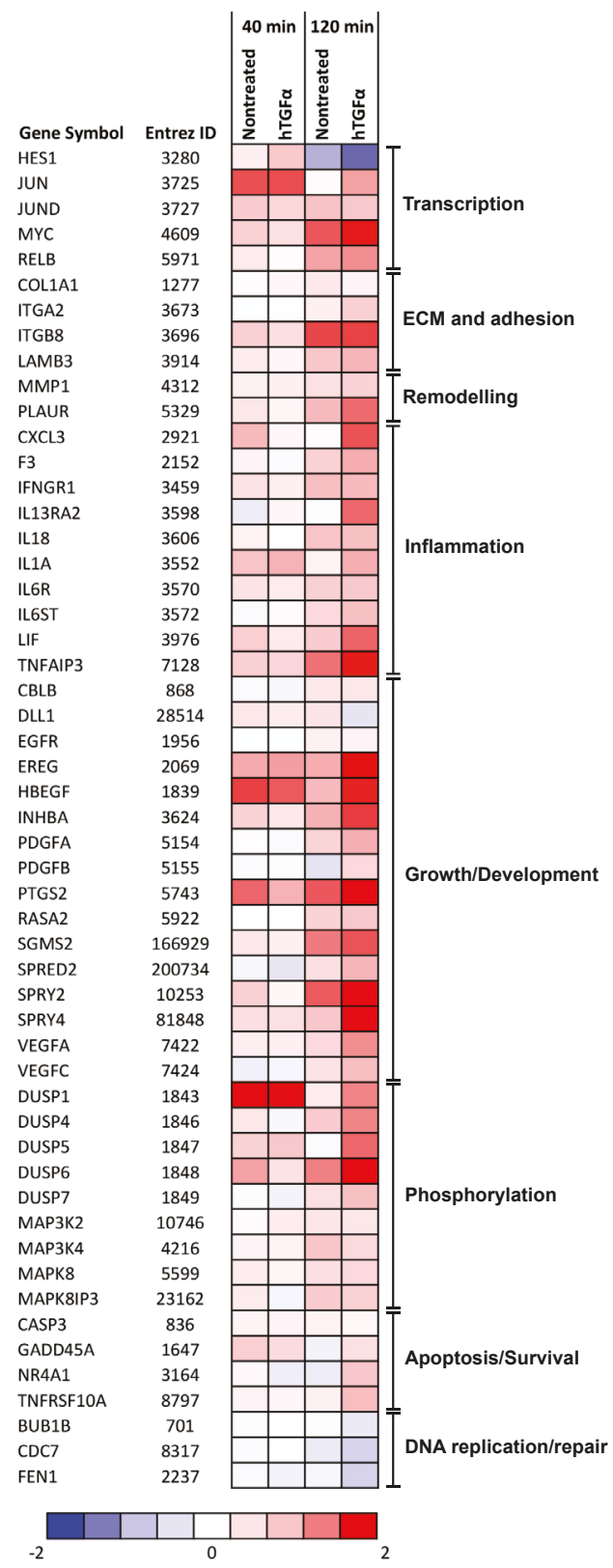

Figure 4. Heatmap displaying log ratios of the differentially expressed genes in the hTGFa-treated cells and non-treated control. Log ratios were calculated relative to the moment of creation of the scratch $(t=0)$ using mean values of three technical replicates. Genes depicted in the heatmap were differentially expressed in the cells exposed to the non-treated control at 120 minutes in relation to the moment of creation of the scratch $(\mathrm{t}=0)$ and/or in hTGFa-treated cells when compared to the non-treated control at 120 minutes (FDR p-value $<0.05$ ). 


\section{A. oris, $A$. viscosus and $V$. parvula reduce stress responses and promote cell survival, whereas $F$. nucleatum subsp. animalis enhances inflammatory and growth-stimulating responses}

The overall effect of the bacteria on the transcriptional responses of gingival cells during re-epithelialization was analysed by PCA using the normalized expression values of the complete array. This analysis showed that after 40 minutes, the transcriptomes of the cells exposed to the bacteria, with the exception of $F$. nucleatum subsp. animalis, clustered together with the non-treated and TGFa-treated controls (Fig. 5), indicating that at this point of the re-epithelialization process the transcriptome responses elicited by the bacterial preparations were quite modest and resemble those of the nontreated control. At a later timepoint (120 minutes), the transcriptomes of cells exposed to the Actinomyces spp. clustered further away from the non-treated control. In contrast, the transcriptome of the cells treated with F. nucleatum subsp. animalis did not cluster with any of the other bacterial preparations or the controls, highlighting the distinct transcriptional responses elicited by F. nucleatum subsp. animalis on the gingival cells.

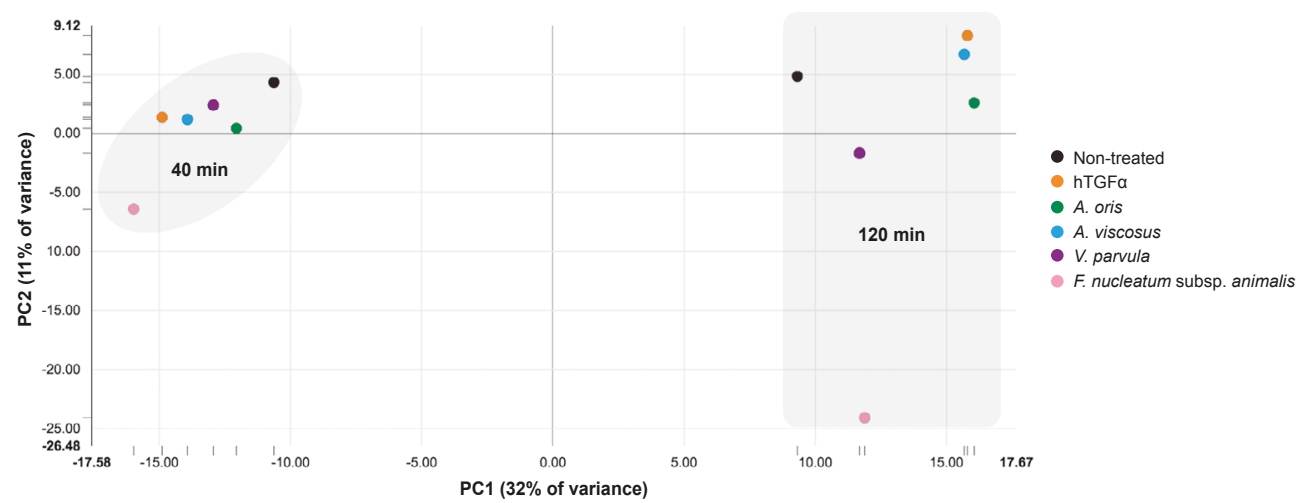

Figure 5. Principal component analysis of the transcriptome of cells exposed to oral commensal bacteria during re-epithelialization. Input data consisted of the mean raw expression values $(n=3)$ representing the transcriptomes obtained from gingival cells exposed to oral commensal bacteria at an $\mathrm{MOI} 100$ at 40 and 120 minutes after introducing a scratch in a confluent cell monolayer.

To identify gene sets associated with the different bacterial treatments, we performed GSEA between the cells exposed to each of these bacteria and the non-treated control at 120 minutes. In line with the results obtained from the PCA, treatment with A. oris, A. viscosus, and V. parvula resulted in similar enriched gene sets (Table S6) and subtle transcriptome responses when compared to the non-treated control. Remarkably, exposure of the gingival cells to the Actinomyces spp. or Veillonella led to significant downregulation of gene sets related to the p53 pathway as well as chromatin organization and nucleosome assembly (Fig S3). Compared with the modest responses elicited with the aforementioned bacteria, $F$. nucleatum subsp. animalis induced transcriptional responses that involved numerous enriched gene sets (Table S7 and Table S8) participating in detection of extracellular and invading microorganisms, inflammation, apoptosis, intracellular signalling, and adaptive immunity. These gene sets included NOD- and Toll-like receptor signalling pathways, cytosolic DNA sensing, cytokine-cytokine receptor interaction, chemokine signalling pathways, MAPK and JAK-STAT signalling pathways, B and T cell receptor signalling, and pathways associated with cancer (Table S7). Downregulated gene sets were related to nitrogen and amino acid metabolism, chromatin organization, and nucleosome assembly (Fig.6). 


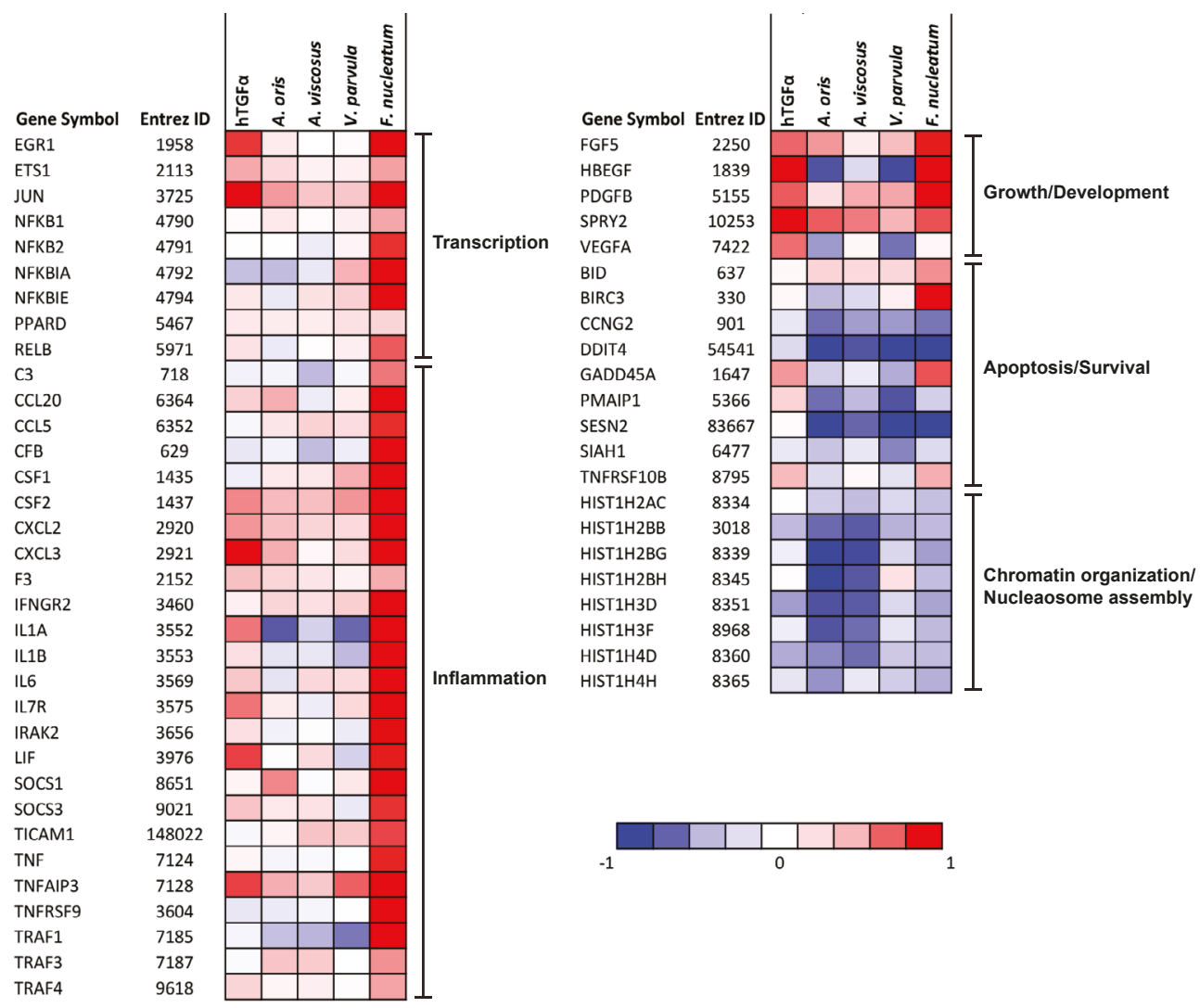

Figure 6. Heatmap displaying log ratios of differentially expressed genes between the bacterial-treated cells and non-treated control at $\mathbf{1 2 0}$ minutes of re-epithelialization. Log ratios were calculated relative to the non-treated control at 120 minutes using mean values of three technical replicates. Genes depicted in the heatmap are a subset of differentially expressed genes in cells treated with F. nucleatum subsp. animalis and/ or cells treated with the Actinomyces spp. or V. parvula (FDR p-value $<0.05$ ).

Cytoscape network analysis constructed with the significantly differentially regulated leading genes from the enriched gene sets showed that the responses of the gingival cells exposed to F. nucleatum subsp. animalis were mainly associated with three central nodes, namely transcription factor ETS1 (ETS1), and c-Jun (JUN), and early growth response 1 (EGR1) (Fig. S4), which act as regulators of re-epithelialization, differentiation, and angiogenesis. Relative to the nontreated control, exposure to F. nucleatum subsp. animalis led to a strong induction of numerous genes participating in inflammatory responses including pro-inflammatory cytokines and chemokines such as colony stimulating factor 2 (CSF2), CXCL3, chemokine (C-C motif) ligand 20 (CCL20), tumor necrosis factor (TNF), IL1A, and IL-6 (Fig. 6, also see Table S9). Finally, the stress-associated gene known as growth arrest and DNA-damage-inducible protein alpha (GADD45A) was upregulated by $F$. nucleatum subsp. animalis, but downlregulated upon treatment with the other commensal species. Similarly, tumor necrosis factor receptor superfamily member 10B (TNFRSF10B), a central regulator of cell death, was induced by F. nucleatum subsp. animalis and downregulated by A. oris and V. parvula. 


\section{Discussion}

Wounds in the oral cavity can result from daily activities (e.g. tooth brushing, chewing, eating) as well as from trauma, surgery, or presence of inflammatory conditions such as periodontal diseases. During active periodontal disease, the epithelial barrier function is disrupted and consequently, bacteria can invade the underlying tissues, resulting in an increased inflammatory response that may lead to the destruction of the periodontal tissues with subsequent reabsorption of the alveolar bone and tooth $\operatorname{loss}^{6,11}$. In a previous study ${ }^{236}$, we found that oral commensal bacteria can influence gingival re-epithelialization in vitro. Importantly, bacterial species-specific effects on re-epithelialization in vitro correlated with in vivo gingival bleeding scores measured in 268 healthy individuals, and this correlation was strongly corroborated when evaluating the same species in healthy individuals that participated in an experimental gingivitis challenge trial ${ }^{236}$. Actinomyces oris, Actinomyces viscosus and Veillonella parvula were identified as the strongest stimulators of reepithelialization, whereas Fusobacterium nucleatum subsp. animalis affected re-epithelialization in a dose-dependent manner, where $\mathrm{MOI} 100$ stimulated while MOI 500 inhibited $^{236}$. In this study, we explored the mechanisms by which strains of $A$. oris, A. viscosus, V. parvula, and F. nucleatum subsp. animalis may modulate gingival re-epithelialization in vitro, using various cell-based assays to quantify relevant aspects of wound healing such as cell migration, production of pro-inflammatory cytokines, and cell viability/death after exposure to the bacteria. These analyses were complemented by transcriptome analyses to explore the transcriptional responses of the gingival cells to epithelial-wounding (i.e. nontreated control) and the treatments with the positive control hTGFa or the bacteria ${ }^{162,237}$.

In our re-epithelialization model, EGFR was one of the central nodes driving the responses of the scratched cell monolayers after 120 minutes. EGFR belongs to the ErbB family of receptor tyrosine kinases (RTKs) that upon activation, triggers the MAPK signalling pathways, which lead to the expression of multiple genes that are responsible for cell proliferation, differentiation, and survival ${ }^{24,250}$, including genes encoding the transcription factors c-Myc and the activator protein 1 (AP-1) subunit JunD, which occurred as two of the main regulators upon wounding. Both transcription factors are important regulators of the processes of cell migration and proliferation involved in the re-epithelialization phase of wound healing ${ }^{69}$. In particular, AP-1 transcription factors regulate the expression of genes encoding metalloproteinases (MMPs) and integrins, which are required for cell migration, as well as growth factors necessary for cell proliferation ${ }^{251}$. This is in agreement with the observation that activation of the EGFR by hTGFa treatment resulted in upregulation of $c-M Y C$ and C-JUN (AP-1 subunit) proto-oncogenes in comparison with the non-treated control. In addition, signalling through the EGFR was tightly regulated by the induction of members of the sprouty/spread family and DUSP proteins. Sprouty and Spread proteins antagonize growth factor-mediated Ras/ERK signalling, downstream of EGFR signalling ${ }^{252,253}$, whereas the DUSP proteins downregulate MAPK signalling by dephosphorylation of the threonine/serine and tyrosine residues required for the activity of these kinases ${ }^{254}$. Additionally, EGFR signalling can activate $\mathrm{Ca}^{2+}$-dependent pathways such as $\mathrm{NF}-\mathrm{KB}$ resulting in expression of pro-inflammatory cytokines and chemokines ${ }^{255}$ as was observed in the transcriptomes of cells exposed to hTGFa.

Once we established the transcriptome responses to the introduction of a wound in the epithelial monolayer (non-treated control) and to hTGFa, we examined the effect of the bacterial panel in comparison with the non-treated control at 120 minutes. Species belonging to the Actinomyces and Veillonella genera are commonly found within different niches of the oral cavity ${ }^{44}$ and have been negatively correlated with clinical parameters related to periodontal diseases in multiple studies ${ }^{223-225,236}$. Compared with the non-treated control, treatment with A. oris, A. viscosus or V. parvula was characterised 


\section{4}

by modest transcriptome responses, with notable downregulation of the cellular stress-induced p53 pathway. Among the downregulated genes was the one coding for GADD45A, which is rapidly induced following stressful growth arrest conditions and treatment with DNA-damaging agent ${ }^{256}$. Likewise, expression of TNFRSF10B was downregulated after treatment with $A$. oris and $V$. parvula. Activation of this receptor results in the formation of the death-inducing signalling complex (DISC) that in turn performs the proteolytic activation of caspase-8, which initiates the cascade of caspases mediated apoptosis ${ }^{257}$. The downregulation of stress- and/or death-related genes by Actinomyces spp. and V. parvula in comparison with the non-treated control may explain the significant increase in cell survival after exposure to these bacteria in our in vitro assays. Furthermore, the moderate gingival epithelial responses elicited by Actinomyces spp. and $V$. parvula support the concept that commensal bacteria have evolved a more balanced relationship with the host $\mathrm{s}^{258,259}$.

F. nucleatum is also a common resident of the oral cavity ${ }^{44}$, but in some individuals it is considered a key microbe in the development of periodontal diseases by recruiting periodontal pathogens such as Porphyromonas gingivalis into subgingival plaque ${ }^{260}$. When compared to the non-treated control at 120 minutes, exposure of gingival cells to F. nucleatum subsp. animalis led to upregulation of more than 60 gene sets (Table S7) including those involved in detection of invading microorganisms such as NOD-like receptor signalling pathway and cytosolic DNA sensing. This finding is in agreement with the previously reported adherence and invasion of $F$. nucleatum in different types of host cells, including gingival epithelial cell ${ }^{261,262}$. Moreover, treatment with $F$. nucleatum subsp. animalis resulted in further enrichment of JAK/STAT

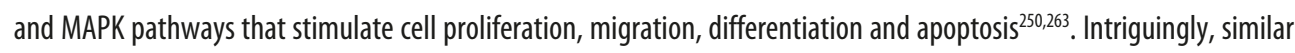
results including MAPK signalling cascade induction were reported by Hasegawa et. al (2007) using human immortalized gingival keratinocytes (HIGK) after exposure for 2 hours to another subspecies, F. nucleatum subsp. nucleatum $(\mathrm{MOI} 100)^{208}$.

In comparison with the non-treated control (baseline), the regulatory network involved in responses to $F$. nucleatum subsp. animalis exposure were mainly driven by three central nodes; (1) c-Jun -which also regulated the responses to hTGFa, (2) ETS1, and (3) EGR1. ETS1 is a transcription factor that is induced by c-Jun via Ras/ERK signaling and plays an important role in a variety of processes such as cell proliferation, differentiation, angiogenesis, and metastasis ${ }^{264-266}$. Moreover, EGR1 has been reported to enhance several aspects of wound healing including re-epithelialization, angiogenesis, and collagen deposition through direct upregulation of growth factors, such as VEGF ${ }^{69}$. Other responses upon incubation of gingival cells with $F$. nucleatum subsp. animalis included a strong induction of pro-inflammatory cytokines when compared to the non-treated control (Table S9). Among the upregulated cytokines, CSF2 and IL1A are involved in epithelial regeneration and cell proliferation ${ }^{267-269}$. Significant induction of IL-6 expresssion and increased levels of protein secretion were observed in the transcriptomes and in the culture media of cells exposed to $F$. nucleatum subsp. animalis in comparison with the non-treated control corroborating the results obtained in an earlier study ${ }^{208}$. Importantly, contrary to the other 'benign' bacterial preparations, F. nucleatum subsp. animalis treatment induced the 'stress sensor' GADD45A and the gene TNFRSF10B that encodes a cell death receptor. The increased stress conditions resulting from enhanced innate immune responses towards $F$. nucleatum subsp. animalis in combination with the induction of cell-death associated genes such as TNFRSF10B, could explain the increased cell death elicited by exposure to this bacterium.

The transwell migration assay revealed that the enhanced re-epithelialization observed with some of the bacterial preparations may involve direct bacterial-host cell interaction. For instance, addition of $F$. nucleatum subsp. animalis and $A$. viscosus in the basolateral compartment did not stimulate cells in the apical compartment to migrate through the porous insert. Conversely, using the same setting, A. oris and V. parvula significantly increased cell migration, suggesting that 
these bacteria may secrete certain components (e.g. small molecules, peptides or proteins) that act as chemoattractant for gingival cells.

Taken together, this study provides insight into the physiologically distinct responses that can be induced or repressed by common oral commensal bacteria in human oral gingival cells. These findings can form the foundation for the development of innovative strategies to support oral health through the maintenance of epithelial integrity and barrier function. Such strategies may include the development of next generation probiotics or (bacterial) bioactive compounds to stimulate re-epithelialization in patients suffering from conditions characterized by impair wound healing such as mucositis and periodontal disease $\mathrm{e}^{77}$.

\section{Acknowledgements}

We would like to acknowledge Dr. Ellen Kranenbarg-Stolte for her help performing the cytokine measurements and Anja Taverne-Thiele for her assistance with the migration assay. Furthermore, we would like to thank Jenny Jansen and Dr. Mark Boekschoten (Human Nutrition, Wageningen University, Wageningen, NL) for processing the RNA samples and performing the microarray. Finally, we want to thank Juri Matualatupauw for the advice and expertise provided regarding the transcriptome analysis and visualization. 


\section{Supplementary data}
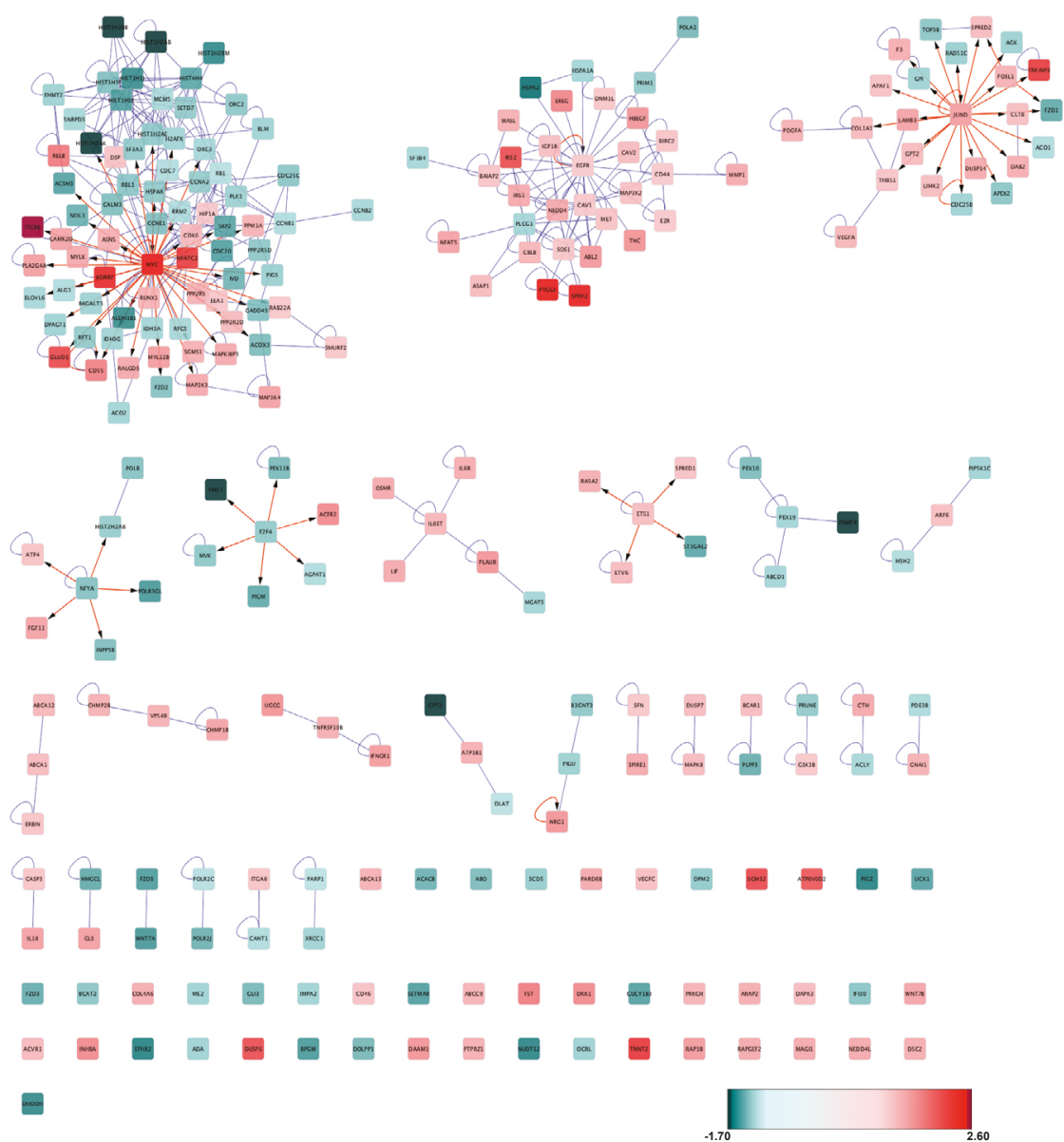

Figure S1. Regulatory network of gingival cells after 120 minutes of re-epithelialization in comparison with the moment of creation of a scratch in a confluent cell monolayer. Interactions of the proteins encoded by the leading genes in the enriched gene sets derived from the comparison between the non-treated control at 120 minutes and $t=0$ (FDR p-value $<0.05)$. Upregulated genes are indicated with a red scale, whereas downregulated genes are indicated with a blue scale. 

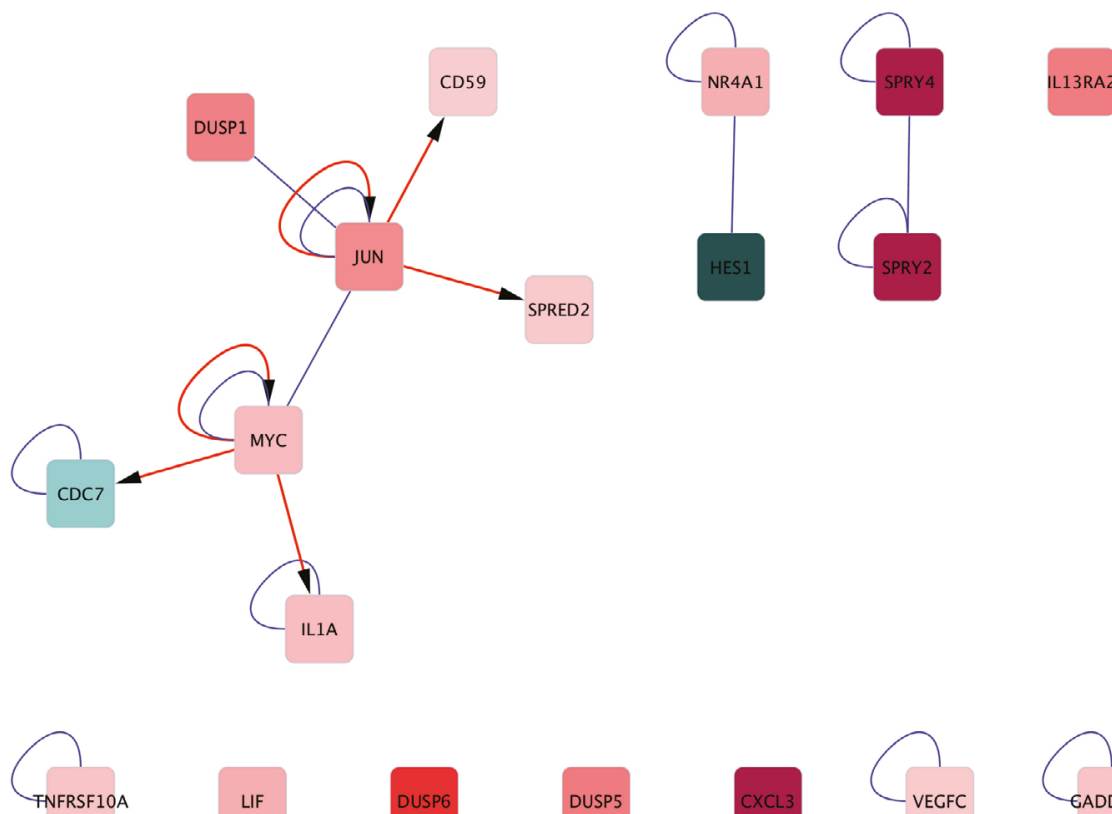

LIF

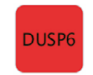

\section{DUSP5}
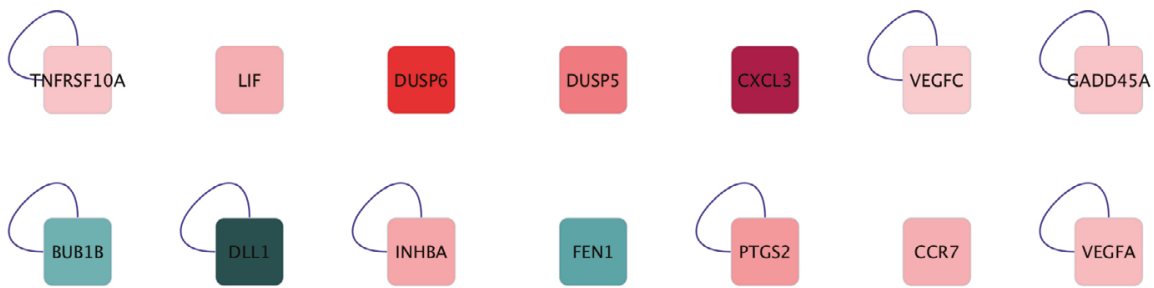

CCR7

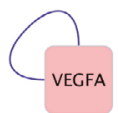

UCCC
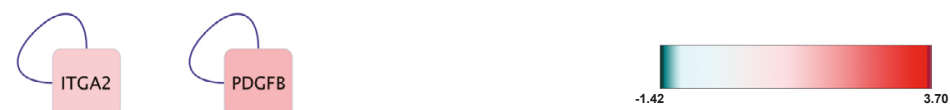

Figure S2. Regulatory network of hTGFa-treated cells in comparison with the non-treated control at 120 minutes during reepithelialization. Interactions of the proteins encoded by the leading genes in the enriched gene sets derived from the comparison between the hTGFa-treated cells and the non-treated control at 120 minutes (FDR p-value $<0.05$ ). Upregulated genes are indicated with a red scale, whereas downregulated genes are indicated with a blue scale. 

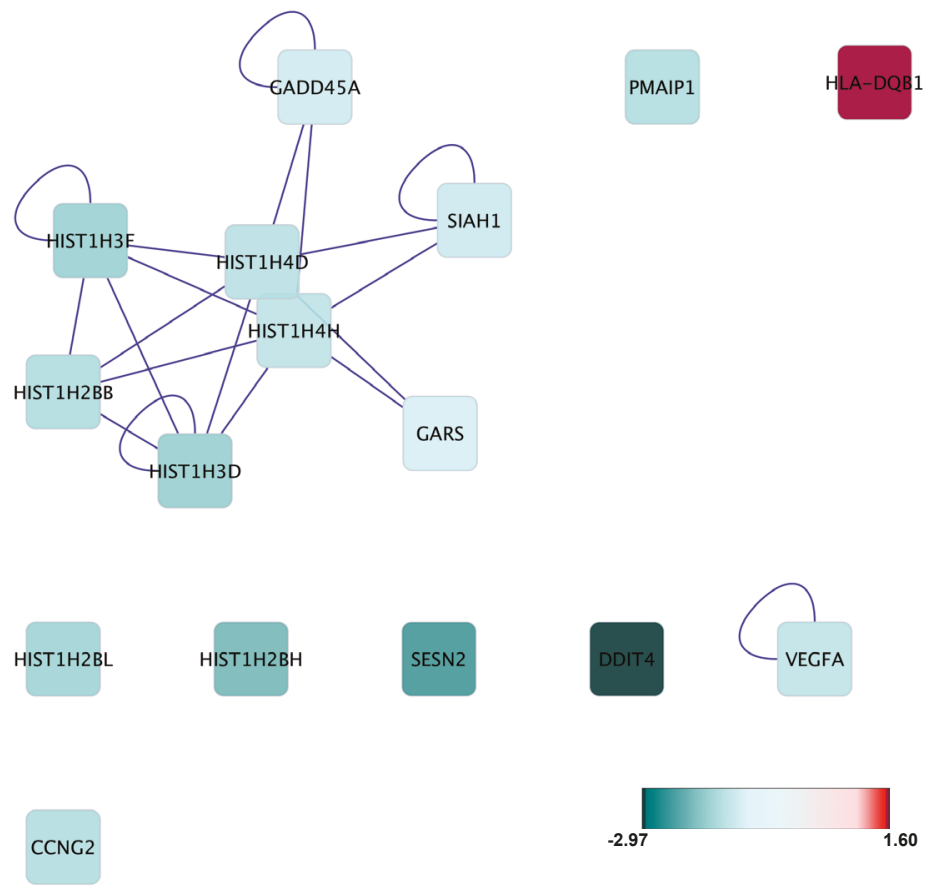

Figure S3. Regulatory network of cells treated with A. oris, A. viscosus or V. parvula in comparison with the non-treated control at 120 minutes during re-epithelialization. Interactions of the proteins encoded by the leading genes in the enriched gene sets derived from the comparison between cells treated with A. oris, A. viscosus or V. parvula and the non-treated control at 120 minutes (FDR p-value $<0.05)$. Genes are overlaid with the expression values obtained for $A$. oris. Upregulated genes are indicated with a red scale, whereas downregulated genes are indicated with a blue scale. 

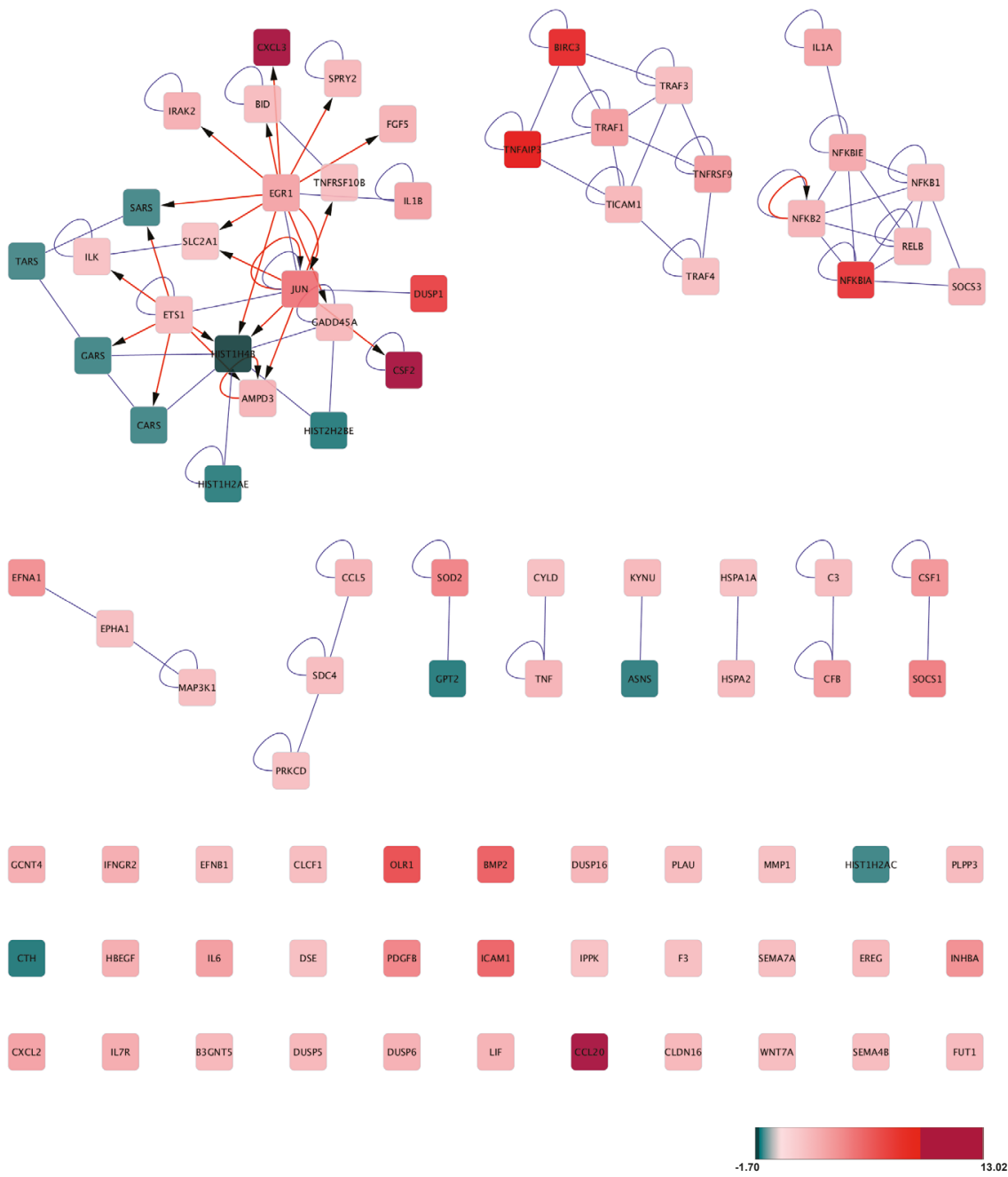

Figure S4. Regulatory network of cells exposed to F. nucleatum subsp. animalis in comparison with the non-treated control at 120 minutes during re-epithelialization. Interactions of the proteins encoded by the leading genes in the enriched gene sets derived from the comparison between the cells exposed to F. nucleatum subsp. animalis (MOI 100) and untreated cells at 120 minutes (FDR p-value $<0.05)$. Upregulated genes are indicated with a red scale, whereas downregulated genes are indicated with a blue scale. 
Table S1. Upregulated gene sets in untreated cells (non-treated control) during the re-epithelialization process. Gene set enrichment analysis (GSEA) was carried out to determine upregulated gene sets in untreated cells 120 minutes after the introduction of the scratch relative to the moment of the introduction of the scratch $(t=0)$ in a confluent cell monolayer.

\begin{tabular}{|c|c|c|c|}
\hline Name & Size & p-value & FDR q-value \\
\hline ECM receptor interaction & 83 & 0.000 & 0.059 \\
\hline Focal adhesion & 197 & 0.000 & 0.066 \\
\hline Sphingolipid metabolism & 38 & 0.000 & 0.081 \\
\hline Arrhythmogenic right ventricular cardiomyopathy (ARVC) & 73 & 0.004 & 0.09 \\
\hline Proximal tubule bicarbonate reclamation & 23 & 0.004 & 0.094 \\
\hline Nitrogen metabolism & 23 & 0.024 & 0.096 \\
\hline MAPK signaling pathway & 263 & 0.000 & 0.102 \\
\hline ErbB signaling pathway & 85 & 0.002 & 0.103 \\
\hline Complement and coagulation cascades & 67 & 0.002 & 0.104 \\
\hline Bladder cancer & 39 & 0.019 & 0.106 \\
\hline JAK-STAT signaling pathway & 146 & 0.00 & 0.111 \\
\hline Alanine aspartate and glutamate metabolism & 32 & 0.02 & 0.113 \\
\hline Type II diabetes mellitus & 46 & 0.008 & 0.119 \\
\hline TGF beta signaling pathway & 84 & 0.010 & 0.12 \\
\hline Hypertrophic cardiomyopathy (HCM) & 82 & 0.017 & 0.147 \\
\hline Chronic myeloid leukemia & 72 & 0.018 & 0.155 \\
\hline Regulation of actin cytoskeleton & 209 & 0.006 & 0.165 \\
\hline Nod-like receptor signaling pathway & 59 & 0.040 & 0.169 \\
\hline Aldosterone regulated sodium reabsorption & 42 & 0.032 & 0.17 \\
\hline Dorso ventral axis formation & 24 & 0.065 & 0.177 \\
\hline $\mathrm{ABC}$ transporters & 44 & 0.039 & 0.185 \\
\hline Cytokine-cytokine receptor interaction & 249 & 0.013 & 0.185 \\
\hline Wnt signaling pathway & 149 & 0.029 & 0.229 \\
\hline Tight-junction & 130 & 0.028 & 0.229 \\
\hline Epithelial cell signaling in Helicobacter pylori infection & 67 & 0.047 & 0.23 \\
\hline Linoleic acid metabolism & 26 & 0.097 & 0.238 \\
\hline Pathways in cancer & 320 & 0.014 & 0.242 \\
\hline Endocytosis & 177 & 0.031 & 0.242 \\
\hline Small cell lung cancer & 83 & 0.063 & 0.248 \\
\hline
\end{tabular}

Table S2. Downregulated gene sets in the untreated cells (non-treated control) during the re-epithelialization process. Gene set enrichment analysis (GSEA) was carried out to determine downregulated gene sets in untreated cells 120 minutes after the introduction of the scratch relative to the moment of the introduction of the scratch $(t=0)$ in a confluent cell monolayer.

\begin{tabular}{lccc}
\hline Name & Size & p-value & FDR q-value \\
\hline Citrate cycle (TCA) cycle & 29 & 0.000 & 0.004 \\
\hdashline Base excision repair & 33 & 0.000 & 0.009 \\
\hline N-glycan biosynthesis & 46 & 0.000 & 0.014 \\
\hline Glycosylphosphatidylinositol (GPI) anchor biosynthesis & 24 & 0.002 & 0.015 \\
\hline Systemic lupus erythematosus & 116 & 0.000 & 0.019 \\
\hline Purine metabolism & 151 & 0.000 & 0.025 \\
\hdashline DNA replication & 35 & 0.002 & 0.030 \\
\hdashline Butanoate metabolism & 34 & 0.006 & 0.049 \\
\hline Propanoate metabolism & 32 & 0.015 & 0.052 \\
\hline
\end{tabular}




\begin{tabular}{|c|c|c|c|}
\hline Name & Size & p-value & FDR q-value \\
\hline RNA polymerase & 27 & 0.008 & 0.056 \\
\hline Progesterone mediated oocyte maturation & 85 & 0.004 & 0.056 \\
\hline Pyrimidine metabolism & 92 & 0.004 & 0.056 \\
\hline Cell cycle & 124 & 0.002 & 0.057 \\
\hline Glycerolipid metabolism & 48 & 0.012 & 0.057 \\
\hline Fatty acid metabolism & 41 & 0.010 & 0.069 \\
\hline Peroxisome & 77 & 0.005 & 0.069 \\
\hline Pyruvate metabolism & 39 & 0.021 & 0.075 \\
\hline Valine, leucine and isoleucine degradation & 44 & 0.015 & 0.075 \\
\hline Glycosaminoglycan biosynthesis keratan sulfate & 15 & 0.041 & 0.098 \\
\hline Spliceosome & 121 & 0.006 & 0.103 \\
\hline Glycolysis gluconeogenesis & 61 & 0.020 & 0.110 \\
\hline Homologous recombination & 28 & 0.041 & 0.111 \\
\hline Antigen processing and presentation & 78 & 0.008 & 0.112 \\
\hline Lysine degradation & 44 & 0.057 & 0.134 \\
\hline Basal cell carcinoma & 55 & 0.041 & 0.135 \\
\hline Mismatch repair & 23 & 0.049 & 0.136 \\
\hline Biosynthesis of unsaturated fatty acids & 22 & 0.078 & 0.168 \\
\hline Oocyte meiosis & 111 & 0.021 & 0.185 \\
\hline Inositol phosphate metabolism & 53 & 0.074 & 0.245 \\
\hline
\end{tabular}

Table S3. Upregulated gene sets in cells treated with hTGFa during the re-epithelialization process. Gene set enrichment analysis (GSEA) was carried out to determine upregulated gene sets in cells treated with hTGFa when compared with untreated cells 120 minutes after the introduction of the scratch.

\begin{tabular}{lccc}
\hline Name & Size & p-value & FDR q-value \\
\hline JAK-STAT signaling pathway & 146 & 0.000 & 0.073 \\
\hline Hematopoietic cell lineage & 83 & 0.000 & 0.084 \\
\hline Cytokine-cytokine receptor interaction & 249 & 0.000 & 0.183 \\
\hline Bladder cancer & 39 & 0.017 & 0.201 \\
\hline Leishmania infection & 67 & 0.015 & 0.206 \\
\hline SNARE interactions in vesicular transport & 36 & 0.018 & 0.207 \\
\hline MAPK signaling pathway & 263 & 0.000 & 0.213 \\
\hline Sphingolipid metabolism & 38 & 0.008 & 0.236 \\
\hline
\end{tabular}

Table S4. Downregulated gene sets in cells treated with hTGFa during the re-epithelialization process. Gene set enrichment analysis (GSEA) was carried out to determine downregulated gene sets in cells treated with hTGFa when compared with untreated cells 120 minutes after the introduction of the scratch.

\begin{tabular}{lccc}
\hline Name & Size & p-value & FDR q-value \\
\hline Glutathione metabolism & 49 & 0.000 & 0.116 \\
\hdashline Systemic lupus erythematosus & 116 & 0.000 & 0.144 \\
\hline Lysosome & 117 & 0.002 & 0.169 \\
\hline Homologous recombination & 28 & 0.032 & 0.174 \\
\hline Progesterone mediated oocyte maturation & 85 & 0.010 & 0.176 \\
\hline Phenylalanine metabolism & 18 & 0.026 & 0.178 \\
\hline Notch signaling pathway & 47 & 0.006 & 0.182 \\
\hline Parkinson's disease & 107 & 0.006 & 0.192 \\
\hline
\end{tabular}




\section{2}

Chapter 7

\begin{tabular}{lccc}
\hline Name & Size & p-value & FDR q-value \\
\hline Oocyte meiosis & 111 & 0.004 & 0.192 \\
\hline Mismatch repair & 23 & 0.021 & 0.196 \\
\hline Cell cycle & 124 & 0.004 & 0.198 \\
\hline Pentose phosphate pathway & 26 & 0.021 & 0.200 \\
\hline Huntington's disease & 165 & 0.000 & 0.201 \\
\hline Nucleotide excision repair & 42 & 0.023 & 0.201 \\
\hline DNA replication & 35 & 0.006 & 0.201 \\
\hline Lysine degradation & 44 & 0.052 & 0.243 \\
\hline
\end{tabular}

Table S5. Differentially expressed genes between hTGFa-treated and untreated cells during re-epithelialization. Differentially expressed leading genes (FDRp-value < 0.05) in the enriched gene sets between hTGFa-treated and untreated cells at 120 minutes. Genes are ranked in descending fold change.

\begin{tabular}{|c|c|c|c|}
\hline Gene symbol & Entrez ID & Fold change & FDR p-value \\
\hline SPRY4 & 81848 & 3.704 & 0.000 \\
\hline CXCL3 & 2921 & 3.697 & 0.001 \\
\hline SPRY2 & 10253 & 3.693 & 0.000 \\
\hline DUSP6 & 1848 & 3.322 & 0.000 \\
\hline DUSP5 & 1847 & 2.262 & 0.000 \\
\hline IL13RA2 & 3598 & 2.248 & 0.000 \\
\hline DUSP1 & 1843 & 2.200 & 0.000 \\
\hline JUN & 3725 & 2.093 & 0.000 \\
\hline PTGS2 & 5743 & 1.908 & 0.001 \\
\hline INHBA & 3624 & 1.744 & 0.001 \\
\hline LIF & 3976 & 1.639 & 0.001 \\
\hline CCR7 & 1236 & 1.615 & 0.024 \\
\hline NR4A1 & 3164 & 1.599 & 0.006 \\
\hline PDGFB & 5155 & 1.534 & 0.032 \\
\hline VEGFA & 7422 & 1.466 & 0.000 \\
\hline MYC & 4609 & 1.442 & 0.001 \\
\hline IL1A & 3552 & 1.434 & 0.000 \\
\hline GADD45A & 1647 & 1.333 & 0.000 \\
\hline TNFRSF10A & 8797 & 1.329 & 0.038 \\
\hline SPRED2 & 200734 & 1.253 & 0.041 \\
\hline VEGFC & 7424 & 1.225 & 0.043 \\
\hline UGCG & 7357 & 1.209 & 0.013 \\
\hline ITGA2 & 3673 & 1.192 & 0.028 \\
\hline CD59 & 966 & 1.190 & 0.040 \\
\hline $\mathrm{CDC7}$ & 8317 & -1.145 & 0.035 \\
\hline BUB1B & 701 & -1.208 & 0.017 \\
\hline FEN1 & 2237 & -1.239 & 0.020 \\
\hline HES1 & 3280 & -1.373 & 0.004 \\
\hline DLL1 & 28514 & -1.418 & 0.000 \\
\hline
\end{tabular}


Table S6. Gene set enrichment analysis of gingival cells exposed to oral commensal bacteria during re-epithelialization. Gene set enrichment analysis (GSEA) was carried out to determine the upregulated and downregulated gene sets in gingival cells exposed to different bacterial preparations when compared to untreated cells after 120 minutes of re-epithelialization. Arrows indicate direction of change ( $\uparrow$, upregulation, $\downarrow$ downregulation).

\begin{tabular}{|c|c|c|c|c|c|c|}
\hline \multirow{2}{*}{ Gene set name } & \multicolumn{2}{|c|}{ Actinomyces oris } & \multicolumn{2}{|c|}{ Actinomyces viscosus } & \multicolumn{2}{|c|}{ Veillonella parvula } \\
\hline & Change & FDR q-value & Change & FDR q-value & Change & FDR q-value \\
\hline Antigen processing and presentation & $\uparrow$ & 0.250 & -- & -- & -- & \\
\hline Systemic lupus erythematosus* & $\downarrow$ & 0.000 & $\downarrow$ & 0.000 & $\downarrow$ & 0.140 \\
\hline mTOR signaling pathway & $\downarrow$ & 0.011 & - & & $\downarrow$ & 0.162 \\
\hline p53 signaling pathway & $\downarrow$ & 0.017 & $\downarrow$ & 0.005 & $\downarrow$ & 0.003 \\
\hline Aminoacyl tRNA biosynthesis & -- & - & - & - & $\downarrow$ & 0.241 \\
\hline
\end{tabular}

Table S7. Upregulated gene sets in cells treated with $F$. nucleatum subsp. animalis during the re-epithelialization process. Gene set enrichment analysis (GSEA) was carried out to determine upregulated gene sets in cells exposed to F. nucleatum subsp. animalis when compared to untreated cells 120 minutes after the introduction of the scratch.

\begin{tabular}{|c|c|c|c|}
\hline Name & Size & p-value & FDR q-value \\
\hline NOD-like receptor signaling pathway & 59 & 0.000 & 0.000 \\
\hline Leishmania infection & 67 & 0.000 & 0.000 \\
\hline Apoptosis & 85 & 0.000 & 0.000 \\
\hline Toll-like receptor signaling pathway & 97 & 0.000 & 0.000 \\
\hline Epithelial cell signaling in Helicobacter pylori infection & 67 & 0.000 & 0.000 \\
\hline Graft versus host disease & 37 & 0.000 & 0.000 \\
\hline Small cell lung cancer & 83 & 0.000 & 0.000 \\
\hline Cytokine-cytokine receptor interaction & 249 & 0.000 & 0.000 \\
\hline T cell receptor signaling pathway & 106 & 0.000 & 0.000 \\
\hline Prion diseases & 34 & 0.000 & 0.000 \\
\hline Hematopoietic cell lineage & 83 & 0.000 & 0.000 \\
\hline Cytosolic DNA sensing pathway & 51 & 0.000 & 0.001 \\
\hline MAPK signaling pathway & 263 & 0.000 & 0.001 \\
\hline B cell receptor signaling pathway & 73 & 0.000 & 0.001 \\
\hline Pathways in cancer & 320 & 0.000 & 0.004 \\
\hline Natural killer cell mediated cytotoxicity & 127 & 0.000 & 0.004 \\
\hline Type I diabetes mellitus & 41 & 0.004 & 0.005 \\
\hline Chemokine signaling pathway & 181 & 0.000 & 0.005 \\
\hline JAK-STAT signaling pathway & 146 & 0.000 & 0.006 \\
\hline Rig I like receptor signaling pathway & 68 & 0.000 & 0.010 \\
\hline Adipocytokine signaling pathway & 65 & 0.002 & 0.010 \\
\hline Prostate cancer & 88 & 0.000 & 0.012 \\
\hline Neurotrophin signaling pathway & 125 & 0.000 & 0.013 \\
\hline Chronic myeloid leukemia & 72 & 0.002 & 0.014 \\
\hline Antigen processing and presentation & 78 & 0.000 & 0.024 \\
\hline Renal cell carcinoma & 67 & 0.002 & 0.026 \\
\hline Viral myocarditis & 66 & 0.004 & 0.039 \\
\hline Focal adhesion & 197 & 0.002 & 0.039 \\
\hline Terpenoid backbone biosynthesis & 15 & 0.020 & 0.041 \\
\hline Steroid biosynthesis & 15 & 0.020 & 0.053 \\
\hline Axon guidance & 128 & 0.004 & 0.054 \\
\hline
\end{tabular}




\begin{tabular}{|c|c|c|c|}
\hline Name & Size & p-value & FDR q-value \\
\hline ECM receptor interaction & 83 & 0.004 & 0.054 \\
\hline 0-glycan biosynthesis & 27 & 0.022 & 0.056 \\
\hline Melanoma & 70 & 0.023 & 0.066 \\
\hline Glioma & 64 & 0.013 & 0.067 \\
\hline Cell adhesion molecules (CAMS) & 131 & 0.005 & 0.070 \\
\hline Ubiquitin mediated proteolysis & 133 & 0.011 & 0.077 \\
\hline Allograft rejection & 35 & 0.020 & 0.078 \\
\hline Inositol phosphate metabolism & 53 & 0.022 & 0.084 \\
\hline Complement and coagulation cascades & 67 & 0.012 & 0.086 \\
\hline ErbB signaling pathway & 85 & 0.027 & 0.105 \\
\hline Peroxisome & 77 & 0.025 & 0.106 \\
\hline Fc epsilon RI signaling pathway & 76 & 0.027 & 0.127 \\
\hline TGF beta signaling pathway & 84 & 0.026 & 0.132 \\
\hline Intestinal immune network for IgA production & 46 & 0.063 & 0.134 \\
\hline PPAR signaling pathway & 66 & 0.038 & 0.135 \\
\hline GnRH signaling pathway & 98 & 0.029 & 0.138 \\
\hline Asthma & 28 & 0.069 & 0.139 \\
\hline Alzheimers disease & 151 & 0.016 & 0.141 \\
\hline Colorectal cancer & 61 & 0.055 & 0.142 \\
\hline Glycosphingolipid biosynthesis lacto and neolacto series & 26 & 0.071 & 0.142 \\
\hline Hedgehog signaling pathway & 56 & 0.056 & 0.148 \\
\hline Regulation of actin cytoskeleton & 209 & 0.017 & 0.148 \\
\hline Dilated cardiomyopathy & 89 & 0.053 & 0.154 \\
\hline Acute myeloid leukemia & 56 & 0.077 & 0.165 \\
\hline Glycerolipid metabolism & 48 & 0.073 & 0.165 \\
\hline Ether lipid metabolism & 31 & 0.108 & 0.168 \\
\hline Glycosaminoglycan biosynthesis chondroitin sulfate & 22 & 0.127 & 0.175 \\
\hline Basal cell carcinoma & 55 & 0.066 & 0.176 \\
\hline Fc gamma R mediated phagocytosis & 91 & 0.061 & 0.179 \\
\hline Purine metabolism & 151 & 0.040 & 0.192 \\
\hline Base excision repair & 33 & 0.114 & 0.198 \\
\hline Tryptophan metabolism & 39 & 0.124 & 0.218 \\
\hline Pancreatic cancer & 69 & 0.111 & 0.245 \\
\hline
\end{tabular}

Table S8. Downregulated gene sets in cells treated with $F$. nucleatum subsp. animalis during the re-epithelialization process. Gene set enrichment analysis (GSEA) was carried out to determine downregulated gene sets in cells exposed to F. nucleatum when compared to untreated cells at 120 minutes during re-epithelialization.

\begin{tabular}{lccc}
\hline Name & Size & p-value & FDR q-value \\
\hline Systemic lupus erythematosus & 116 & 0.000 & 0.065 \\
\hline Nitrogen metabolism & 23 & 0.020 & 0.150 \\
\hline Alanine aspartate and glutamate metabolism & 32 & 0.027 & 0.189 \\
\hline
\end{tabular}


Table S9. Differentially expressed genes between cells exposed to $F$. nucleatum subsp. animalis and untreated cells during reepithelialization. Differentially expressed leading genes (FDR p-value $<0.05$ ) in the enriched gene sets between F. nucleatum subsp. animalis-treated and untreated cells at 120 minutes. Genes are ranked in descending fold change.

\begin{tabular}{|c|c|c|c|}
\hline Gene Symbol & Entrez ID & Fold change & FDR p-value \\
\hline CSF2 & 1437 & 13.019 & 0.000 \\
\hline CXCL3 & 2921 & 10.372 & 0.000 \\
\hline CCL20 & 6364 & 9.046 & 0.000 \\
\hline TNFAIP3 & 7128 & 8.817 & 0.000 \\
\hline BIRC3 & 330 & 7.815 & 0.000 \\
\hline NFKBIA & 4792 & 7.151 & 0.000 \\
\hline DUSP1 & 1843 & 6.543 & 0.000 \\
\hline OLR1 & 4973 & 6.070 & 0.000 \\
\hline BMP2 & 650 & 5.313 & 0.000 \\
\hline ICAM1 & 3383 & 5.098 & 0.000 \\
\hline JUN & 3725 & 4.277 & 0.000 \\
\hline SOCS1 & 8651 & 3.995 & 0.000 \\
\hline PDGFB & 5155 & 3.822 & 0.000 \\
\hline SOD2 & 6648 & 3.817 & 0.000 \\
\hline INHBA & 3624 & 3.368 & 0.000 \\
\hline EFNA1 & 1942 & 3.321 & 0.000 \\
\hline CSF1 & 1435 & 3.075 & 0.000 \\
\hline TNFRSF9 & 3604 & 2.845 & 0.000 \\
\hline IL-6 & 3569 & 2.758 & 0.000 \\
\hline TRAF1 & 7185 & 2.706 & 0.000 \\
\hline CFB & 629 & 2.675 & 0.000 \\
\hline CXCL2 & 2920 & 2.577 & 0.009 \\
\hline IL1B & 3553 & 2.552 & 0.000 \\
\hline IL1A & 3552 & 2.515 & 0.000 \\
\hline EGR1 & 1958 & 2.461 & 0.000 \\
\hline NFKBIE & 4794 & 2.233 & 0.003 \\
\hline IL7R & 3575 & 2.174 & 0.001 \\
\hline HBEGF & 1839 & 2.065 & 0.000 \\
\hline IFNGR2 & 3460 & 2.030 & 0.000 \\
\hline GCNT4 & 51301 & 2.009 & 0.000 \\
\hline IRAK2 & 3656 & 1.963 & 0.000 \\
\hline DUSP6 & 1848 & 1.949 & 0.001 \\
\hline FGF5 & 2250 & 1.852 & 0.005 \\
\hline LIF & 3976 & 1.849 & 0.000 \\
\hline TNF & 7124 & 1.807 & 0.000 \\
\hline CCL5 & 6352 & 1.745 & 0.001 \\
\hline NFKB2 & 4791 & 1.732 & 0.000 \\
\hline DUSP16 & 80824 & 1.723 & 0.000 \\
\hline AMPD3 & 272 & 1.714 & 0.001 \\
\hline SOCS3 & 9021 & 1.710 & 0.005 \\
\hline DUSP5 & 1847 & 1.648 & 0.000 \\
\hline B3GNT5 & 84002 & 1.632 & 0.000 \\
\hline CLDN16 & 10686 & 1.626 & 0.023 \\
\hline TICAM1 & 148022 & 1.617 & 0.034 \\
\hline
\end{tabular}




\begin{tabular}{|c|c|c|c|}
\hline Gene Symbol & Entrez ID & Fold change & FDR p-value \\
\hline KYNU & 8942 & 1.599 & 0.017 \\
\hline EREG & 2069 & 1.597 & 0.005 \\
\hline SPRY2 & 10253 & 1.574 & 0.023 \\
\hline GADD45A & 1647 & 1.568 & 0.000 \\
\hline RELB & 5971 & 1.533 & 0.005 \\
\hline HSPA2 & 3306 & 1.516 & 0.000 \\
\hline PPAP2B & 8613 & 1.506 & 0.002 \\
\hline FUT1 & 2523 & 1.496 & 0.048 \\
\hline SEMA4B & 10509 & 1.432 & 0.001 \\
\hline C 3 & 718 & 1.430 & 0.019 \\
\hline PLAU & 5328 & 1.394 & 0.007 \\
\hline CLCF1 & 23529 & 1.389 & 0.004 \\
\hline SDC4 & 6385 & 1.381 & 0.000 \\
\hline WNT7A & 7476 & 1.368 & 0.001 \\
\hline BID & 637 & 1.359 & 0.009 \\
\hline TRAF3 & 7187 & 1.354 & 0.008 \\
\hline SEMA7A & 8482 & 1.348 & 0.006 \\
\hline MAP3K1 & 4214 & 1.321 & 0.000 \\
\hline EFNB1 & 1947 & 1.312 & 0.004 \\
\hline ETS1 & 2113 & 1.305 & 0.008 \\
\hline TRAF4 & 9618 & 1.297 & 0.050 \\
\hline DSE & 29940 & 1.290 & 0.005 \\
\hline NFKB1 & 4790 & 1.285 & 0.000 \\
\hline EPHA1 & 2041 & 1.280 & 0.029 \\
\hline F3 & 2152 & 1.267 & 0.001 \\
\hline TNFRSF10B & 8795 & 1.265 & 0.042 \\
\hline HSPA1A & 3303 & 1.254 & 0.015 \\
\hline PRKCD & 5580 & 1.235 & 0.011 \\
\hline CYLD & 1540 & 1.230 & 0.026 \\
\hline MMP1 & 4312 & 1.230 & 0.024 \\
\hline ILK & 3611 & 1.198 & 0.008 \\
\hline IPPK & 64768 & 1.197 & 0.023 \\
\hline SLC2A1 & 6513 & 1.166 & 0.012 \\
\hline TARS & 6897 & -1.142 & 0.027 \\
\hline SARS & 6301 & -1.152 & 0.024 \\
\hline CARS & 833 & -1.175 & 0.034 \\
\hline GARS & 2617 & -1.182 & 0.002 \\
\hline HIST1H2AC & 8334 & -1.210 & 0.029 \\
\hline ASNS & 440 & -1.278 & 0.001 \\
\hline HIST1H2AE & 3012 & -1.294 & 0.015 \\
\hline HIST2H2BE & 8349 & -1.321 & 0.004 \\
\hline GPT2 & 84706 & -1.334 & 0.005 \\
\hline CTH & 1491 & -1.357 & 0.001 \\
\hline HIST1H4B & 8366 & -1.698 & 0.001 \\
\hline
\end{tabular}




\section{Chapter}

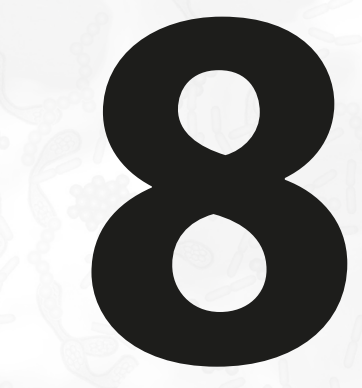

Discussion 


\section{Introduction}

The work presented in this thesis was part of a larger, integrated project entitled "Novel Strategies to Promote Oral Health" that was governed by TIFN and allowed the connection of the in vitro study data to in vivo information collected in healthy volunteers. This thesis focusses on the interactions between the oral mucosa and different components of the oral ecosystem (i.e. salivary metabolites and oral commensal bacteria) that may influence re-epithelialization kinetics, a key aspect for maintenance of epithelial integrity and barrier function. The discussion is divided in separate sections that deal with (1) epithelial integrity and barrier function technology development, (2) understanding the mucosal interactions in the oral ecosystem, and (3) a perspective on oral health.

\section{Epithelial integrity and barrier function technology development}

Oral wounds can result from daily activities (e.g. chewing, eating, tooth brushing and flossing), as well as from trauma, surgery or presence of oral diseases such as oral mucositis and periodontitis $5^{6,77}$. Thus, constant renewal and repair are necessary to maintain the architectural integrity of the oral epithelium. When epithelium integrity is compromised, the processes involved in wound healing are immediately initiated to re-establish cell-cell contacts through reepithelialization ${ }^{69,70}$. As we showed in this thesis, oral re-epithelialization can be influenced by different components of the oral ecosystem, including commensal bacteria (Chapters 2, 6-7) and salivary constituents (Chapter 5). To begin to understand the individual role of each of these components on oral re-epithelialization, it is necessary to employ a reductionist approach. Therefore, we developed and standardized a high-throughput screening model to measure reepithelialization in vitro (Chapters 2-4).

\section{High-throughput scratch assay: A reductionist approach to study re-epithelialization}

Scratch assays mimic the pattern of cell migration observed in vivo (e.g. collective cell migration) and therefore, they have been frequently employed to study the influence of bioactive substances on re-epithelialization ${ }^{91,103,147}$. These assays are carried out in multi-well plates and commonly rely on the manual introduction of a scratch into a confluent cell monolayer that is monitored by acquiring images immediately after wounding and at regular intervals during reepithelialization ${ }^{103,148}$. The scratch is usually created one well at the time using a pipette tip or other sharp tool ${ }^{103,160}$, limiting the throughput and reproducibility of the assay. In addition, re-epithelialization data analysis often lacks realtime kinetic data (e.g. repair rate) or single cell migration analyses ${ }^{103,119}$. To overcome these limitations, we developed a high-throughput scratch assay using live-automated fluorescence microscopy and image segmentation, which is coupled to a mathematical model that describes the kinetics of re-epithelialization (Chapter $\mathbf{2}^{155}$ ). We increased the throughput and reproducibility of the scratches by using 96-well plates in combination with the HTSScratcher (Peira) to ensure scratch uniformity.

Effective processing and quantitative analyses of high-throughput image-datasets are not always straightforward and often require the integration of multiple image processing and data extraction software tools. We developed a cellrecognition pipeline using CellProfiler ${ }^{150}$ that was designed to recognise and record the location of single cells within each well throughout the image-series of a single well. To visualize the extracted information and link it back to the 
raw images, we purchased a license for FCS Express 4 Plus (De Novo Software, CA, USA) ${ }^{152}$, which allowed automated enumeration of cells entering the scratched area in each well over time. To avoid such software license costs we developed custom-made scripts (Chapter 3) written in open-source programming languages (e.g. Perl, R) for automated image segmentation and data analysis ${ }^{149}$. The sigmoidal curves that result from plotting the number of cells infiltrating the scratched area over time were modelled using the modified Gompertz function ${ }^{105}$ (Chapter $\mathbf{2}^{155}$ ). The iterative method used for modelling yielded excellent fits and enabled the extraction of three biologically comprehensive parameters $\left(\mu_{\mathrm{m}^{\prime}}\right.$ $A$ and $\lambda$ ) that describe re-epithelialization kinetics.

Appropriate controls are essential to improve data reliability of high-throughput assays. By optimising the concentration of the controls used in our assay, we were able to obtain a large dynamic range between the positive and negative controls as determined by the $Z^{\prime}$ factor ${ }^{106}$, which together with the combinatorial approach of the repair rate $\left(\mu_{m}\right)$ and the maximum number of cells $(A)$ in a 'performance value' $\left(\mu_{m}{ }^{*} A\right)$, allowed the efficient identification of potential stimulators and attenuators of oral re-epithelialization.

Taken together, standardisation of the experimental variables, well-defined procedures for the assay, and appropriate software tools for data analyses are crucial to achieve high reproducibility and allow meaningful comparisons among different studies. Chapters $\mathbf{3}$ and $\mathbf{4}$ aimed to facilitate data analysis of high-throughput scratch assays and to provide a standardised protocol for the scientific community using cell lines from different origins.

\section{Standardized high-throughput scratch assay for the broader scientific community}

The Kinetic Re-Epithelialization Analysis Pipeline (KREAP) implemented in Galaxy, provides an open-source, web-based platform for efficient, reproducible, and quantitative analysis of high-throughput scratch assays (Chapter 3). The workflow integrates different validated tools for image segmentation, visualization, and data analysis, which is provided as a virtual machine (VM) containing a fully operational KREAP Galaxy installation. After installation of the VM, the user needs to upload the raw images derived from a time series experiment together with an index file of the multi-well plate, and press the Execute button. The KREAP toolbox integrates the cell-recognition pipeline for automated image segmentation designed in Chapter $\mathbf{2}^{155}$ into the workflow of the "Image Analysis" tool. Following image processing, a custom-made script written in $\mathrm{R}$ was implemented in the tool for automatic detection of the scratch boundaries and enumeration of the cells inside and outside the scratch. Lastly, the output of the "Image Analysis" tool models the reepithelialization curve obtained in every well and extracts the kinetic parameters derived from the modified Gompetz function. Galaxy's graphical user interface provides an organized and user-friendly view of the output, which is saved in the Galaxy environment for future consultation. Importantly, we confirmed that the kinetic parameter values obtained with KREAP strongly correlate with the values obtained using the multi-software approach employed in Chapter $\mathbf{2}^{155}$. Moreover, the time required to analyse a total of 960 images was reduced from 3 or 4 hours to less the 30 minutes.

A step-by-step standardized procedure for the assay set-up and KREAP data analysis was provided in Chapter 4. There, we described the optimized experimental variables for two cell lines originating from the oral cavity (i.e. Ca9-22 and H0-1-N-1) and one originating from skin, HaCat. Selection of a suitable cell line for the assay is among the most critical steps to guarantee the success of the experiment and a cell line-specific set of experimental variables must be optimized accordingly. These variables include: seeding density, duration of starvation (i.e. FCS deprivation) prior to the experiment, media used during starvation, optimal concentrations of the controls, and imaging time. Cell lines such as Caco-2 and HT- 
29, derived from colorectal adenocarcinomas, have a doubling time of more than 60 hours and can form tight-junctions. These characteristics proved not to be ideal for the assay because of the long imaging periods required to achieve full wound closure and the irregularity of the scratches due to strong cell-cell adherence (data not shown). It is also important to first evaluate the expression of relevant receptors in the candidate cell lines as well as their responsiveness towards microbial and /or chemical stimuli. It is estimated that $15-30 \%$ of all cell lines are contaminated with mycoplasma ${ }^{270}$, which affect cells by changing protein-composition, metabolism, signal transduction, proliferation characteristics, and inducing chromosomal aberrations ${ }^{271}$. Therefore, to ensure validity of the results obtained with the assay, the cell lines should be routinely checked for mycoplasma infection ideally using PCR-based methods that provide fast, specific and sensitive detection ${ }^{272}$.

\section{Future Perspectives}

\section{Understanding molecular interactions}

The screening model presented in this thesis can be used not only to study the influence of particular substances on reepithelialization, but also allows further investigations into the mechanisms underlying the re-epithelialization effects observed (Chapter $\mathbf{2}^{155}$ ). The use of specific chemical inhibitors or blocking antibodies can be employed to investigate the involvement of specific cellular factors. For example, the specific inhibitors of p38 (SB203580) and MEK1/2 (U0126) can dissect the involvement in these two pathways that are activated by EGFR (Chapter 4, Box 1), although one has to be cautious in interpreting the impact of either one of these inhibitors, because it is well established that there is significant cross-talk between the $\mathrm{p} 38$ and MEK1/2 pathways ${ }^{273}$. Nevertheless, this example illustrates how the inclusion of specific inhibitory chemicals can be employed in the re-epithelialization assay to verify the involvement of particular cellular pathways or components. Analogously, blocking antibodies have been instrumental to establish the participation of specific cell-surface receptors and can be included in the medium during the re-epithelialization assay. However, caution should be taken concerning the stability of these antibodies that should remain functional during the assay, which could be particularly challenging when evaluating the effect of bacteria producing high amounts of proteolytic enzymes. First steps towards identification of specific factors involved in mediating bacterial-treatment effects can be obtained using cellular response profiling methods, such as transcriptomics (Chapter 7), which can facilitate hypothesis building and can serve to design subsequent experiments using specific inhibitors to verify such hypotheses. For example, based on the findings in Chapter 7, one could consider employing AKT inhibitor VII274, an allosteric Akt kinase inhibitor, to establish its role in the differential capacity of $A$. oris, A. viscosus, V. parvula, and F. nucleatum subsp. animalis to modulate cell survival. In addition, transcriptomics can be complemented with phospho-proteomics studies, which would allow the detection of effector proteins that are activated upon phosphorylation. Recent developments such as PamChip arrays (PamGene, 's-Hertogenbosch, NL) or multiplexed ion beam imaging (MIBI) techniques ${ }^{275}$ are interesting options to conduct such studies. PamChip arrays allow kinetic analysis of enzymatic reactions of kinases present in a sample ${ }^{276}$, whereas MIBI uses secondary ion mass spectrometry in combination with antibodies tagged to reporters to image up to 100 targets simultaneously ${ }^{275}$.

Creation of specific knockdown or knockout cell line derivatives provides an alternative strategy to establish the involvement of a specific factor in the effects elicited by a particular treatment. To this end, RNA interference (RNAi) 
and genome-editing approaches using the CRISPR-Cas9 system are effective strategies to investigate the involvement of specific genes in the responses triggered ${ }^{277,278}$. The use of CRISPR-Cas9 technology is particularly attractive because of its versatility, which is based on the easy design of single guide RNAs (sgRNA) that direct the nuclease Cas9 to a protospaceradjacent motif (PAM; 5'-NGG-3' in the case of the frequently used Streptococcus pyogenes (as9) flanking the $3^{\prime}$ end of a target site in the genome ${ }^{278}$. Upon target-binding, Cas9 induces a double strand DNA break that cells commonly repair by non-homologous end-joining (NHEJ) that in general leaves an irreversible mutation (indel) at the target site causing a knockout of the targeted gene ${ }^{279}$. To illustrate the potential of this approach we designed a strategy to knockout Toll-like receptor 5 (TLR5) in Ca9-22 cells (Fig. 1a). Cells were transfected with pX330 plasmids ${ }^{280}$ expressing a human codonoptimized S. pyogenes Cas9 and a sgRNA targeting the forth exon of the TLR5 gene (Table 1), intending to create a TLR5 knockdown cell population. After transfection, cells were expanded and treated with $100 \mathrm{ng} / \mathrm{ml}$ flagellin, which acts as the TLR5 agonist and activates the production of pro-inflammatory cytokines ${ }^{281}$, showing that the resulting TLR5 knockdown cell population produced significantly less interleukin 8 (IL-8) as compared to the wild-type Ca9-22 cells (Fig. 1b). This finding indicates that at least a significant part of the transfected cell population was TLR5 deficient. To obtain a pure knockout population, it would be necessary to use alternative Cas9-sgRNA expressing vectors that also express fluorescent proteins (e.g. pU6 vector, Addgene plasmid \#64218) or selectable antibiotic markers (e.g. pSpCas9(BB)-2APuro (PX459) V2.0, Addgene plasmid \#62988) that enable screening and selection of transfected (TLR5) knockout cells.

Table 1. Design of sgRNA sequences targeting the TLR5 gene. The nucleotides depicted in lowercase correspond to the target sequence, whereas the nucleotides in uppercase were added for ligation into the pX330 vector. On-target and off-target scores are provided along with the PAM motif.

\begin{tabular}{|c|c|c|c|c|}
\hline TLR5 sgRNAs & Sequence $\left(5^{\prime} \rightarrow 3^{\prime}\right)$ & On-target & Off-target & PAM \\
\hline sgRNA1_FW & CACCGctgcagctgttccagaaag & \multirow{2}{*}{69} & \multirow{2}{*}{31} & \multirow{2}{*}{ GGG } \\
\hline sgRNA1_RV & AAACctttctggaacagctgcagC & & & \\
\hline sgRNA2_FW & CACCGctcctaggagaaggtccagg & \multirow{2}{*}{68} & \multirow{2}{*}{34} & \multirow{2}{*}{ TGG } \\
\hline SgRNA2_RV & AAACcctggaccttctcctaggagC & & & \\
\hline
\end{tabular}

The Cas9 driven genome editing system can also be employed to create knock-in cell line derivatives. This is especially of interest when considering the construction of stable knock-in reporter cell lines that allow quantification of expression of selected gene(s) that are of interest for re-epithelialization. Candidate genes for such reporter strategy could include those encoding proliferation markers such as Ki-67282 or pro-inflammatory cytokines such as IL-6 and IL-8. The creation of such reporter cell lines would add an additional layer of resolution to the re-epithelialization assay, by providing a single cell quantification of the level of activation of the selected gene(s). To obtain such reporter cell lines, cells need to be co-transfected with a Cas9/sgRNA expression vector and a repair template. The repair template should contain a suitable reporter gene (e.g. a fluorescent protein coding sequence) and homologous flanks for the locus in which the reporter gene is to be inserted. Following the introduction of the double strand break by Cas9, the repair template can be integrated at the target locus to repair the double strand break by homologous recombination (homology-directed repair [HDR]) within the flanking regions present in the repair template, thereby, effectively delivering the reporter gene at the chromosomal target location ${ }^{283}$. However, the greatest challenge in this knock-in strategy, is the relatively low efficiency of HDR relative to NHE ${ }^{284}$. Recent studies have shown that it is possible to promote HDR at the expense of NHEJ by inhibition of keyfactors involved in NHEJ such as DNA ligase IV (Scr7 inhibitor) ${ }^{285,286}$, which offers approaches to enhance the knock-in frequency. Knock-in reporter cell lines would greatly facilitate the monitoring and quantification of specific biological 
processes or signalling pathways in single cells or populations of cells using high-throughput, high-content microscopy. In the context of re-epithelialization assays, well-chosen knock-in reporters could provide direct information about the involvement of certain pathways or responses in the re-epithelialization impacts observed with a specific treatment.

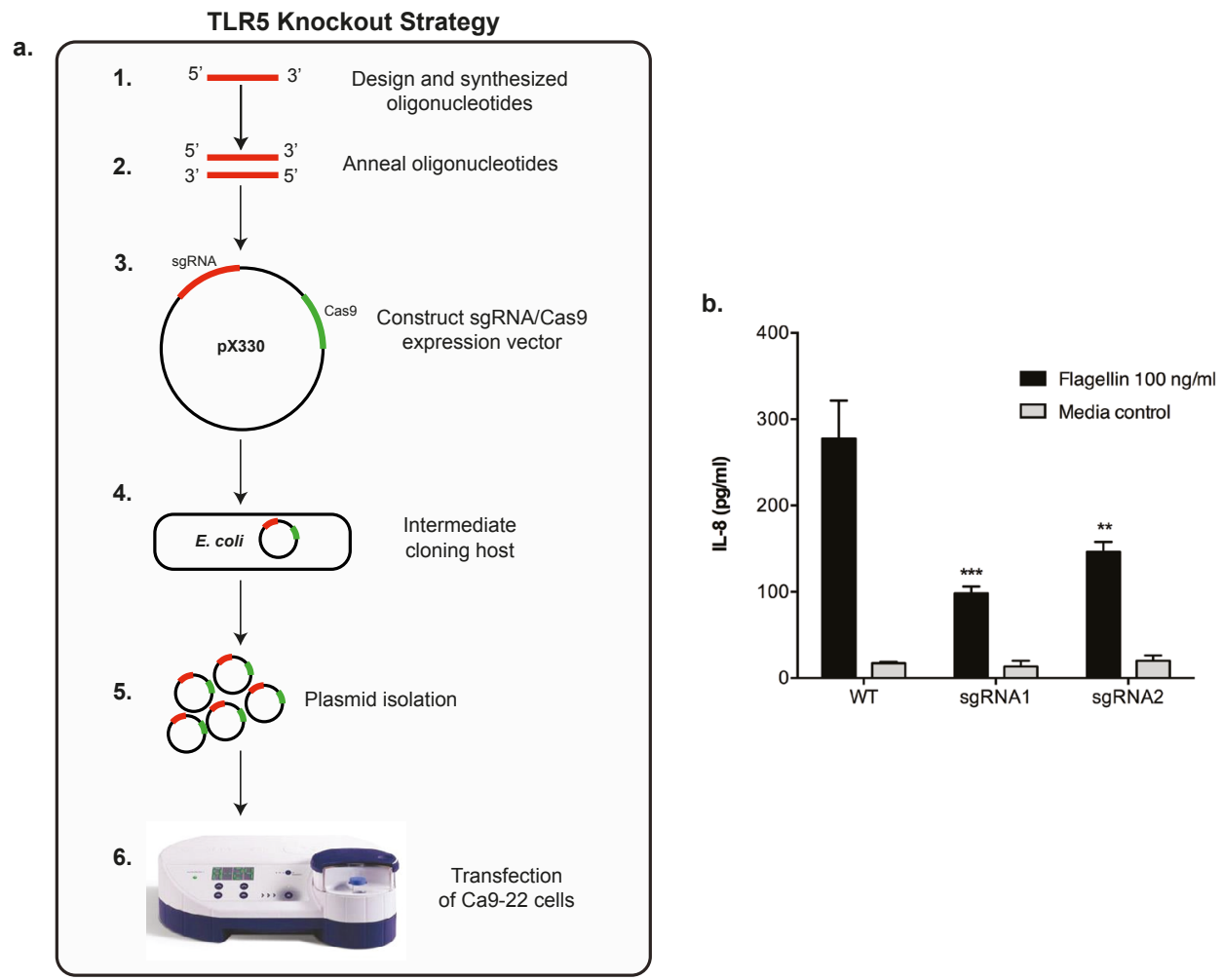

Figure 1. CRISPR-Cas9 genome editing strategy. (a) Strategy followed to knockout TLR5 in Ca9-22 cells using CRISPR-Cas9, based on the protocol developed by Zhang's lab ${ }^{280}$. (1) sgRNA targeting two sites of the TLR5 gene were designed in silico using Benchling designing tool (https://benchling. com), and synthesized by Eurogentec (Maastricht, NL). (2) The oligonucleotides were phosphorylated, annealed, and (3) cloned into the pX300 vector which expresses a human codon-optimized S. pyrogenes Cas9 and the sgRNA targeting the TLR5 gene. (4) Competent $E$. coli were transformed with the pX330 vector and plated in Luria-Bertani (LB) agar containing $100 \mu \mathrm{g} / \mathrm{ml}$ ampicillin and incubated at $37^{\circ} \mathrm{C}$. (5) Positive transformants were grown overnight in LB medium with antibiotics, after which plasmids were isolated. (6) Ca9-22 cells were transfected with the constructs using Amaxa nucleofection (Lonza, Cologne, DE). (b) Concentration of IL-8 in epithelial cell supernatants after 7 hours incubation with or without $100 \mathrm{ng} / \mathrm{ml}$ flagellin. Ca9-22 cells were transfected with the construct expressing two different sgRNAs targeting the TLR5 gene. WT: wild-type Ca9-22 cells. Significant differences from the wildtype cells were assessed by a one-way ANOVA using Dunnett's test for multiple comparisons (**, $P<0.01,{ }^{* * *}, \mathrm{P}<0.0001 ; \mathrm{n}=3$ ).

\section{Barrier function studies}

An important function of the oral epithelium, especially in the gingival sulcus area, is to protect and prevent the body from microbial invasion by providing a tight barrier. Gingival keratinocytes are connected to each other by gap junctions, desmosomes, and tight junctions ${ }^{287}$. Tight junctions limit paracellular and intramembrane diffusion of soluble molecules and play an important role in regulating cell differentiation, proliferation and polarization ${ }^{288,289}$. In addition, tight junctions interact with the actin cytoskeleton ${ }^{290}$, providing strong connections between cells. A measurable indicator of 
barrier function in vitro is the ability to form transepithelial electrical resistance (TEER) ${ }^{287}$. Next to our re-epithelialization studies it is also important to address the re-establishment of appropriate epithelial barrier function after a wound is closed. Unfortunately, the cell line we employed (i.e. (a9-22) does not form a tightly connected cell monolayer and fails to establish a TEER. To overcome this gap in our possibilities to measure epithelial function, we initiated the establishment of a high-throughput barrier function assay to study the influence of specific treatments, e.g. oral commensal bacteria or saliva, on gingival epithelial barrier function. To the best of our knowledge, the only oral cell line capable of expressing tight junctions is the immortalized human gingival keratinocyte cell line (IHGK) that was established by Gröger et al. ${ }^{291}$.
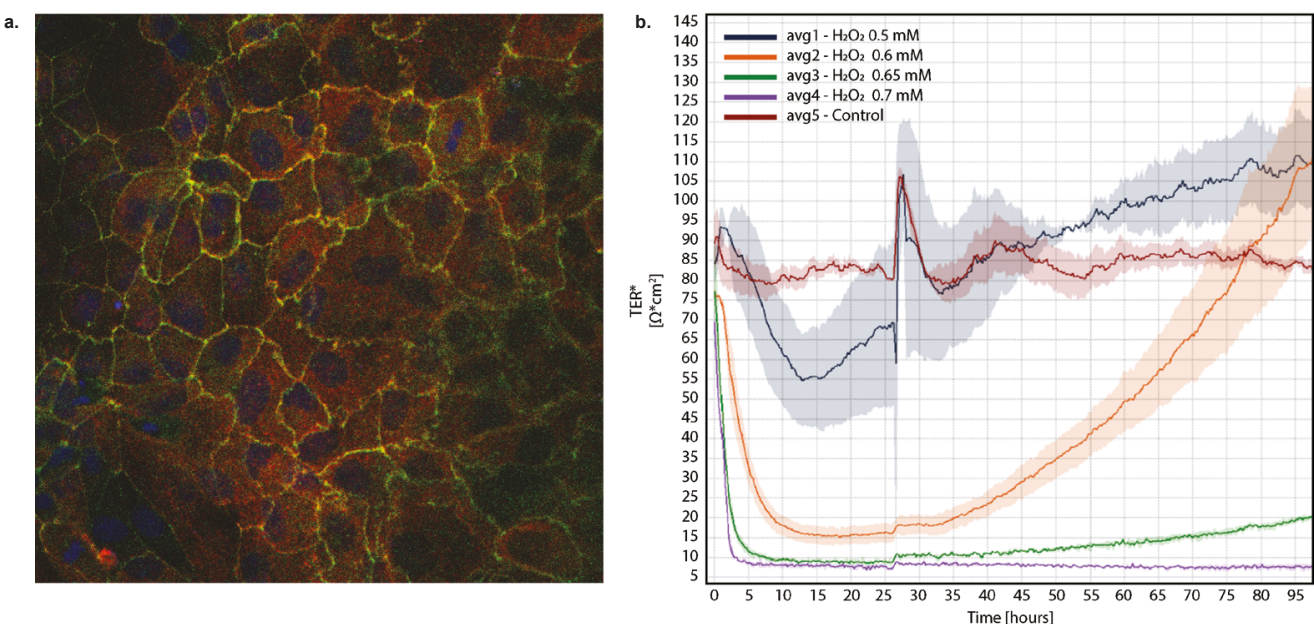

Figure 2. Human immortalized gingival keratinocytes. (a) Cells were grown for one week on transwell inserts after which the cells were fixed and permeabilized with $4 \%$ paraformaldehyde (PFA) and 0.1\% Triton, respectively. Cells were stained with antibodies directed against occludin and Z0-1, which were coupled to Alexa Fluor ${ }^{\oplus} 488$ (green) and Cy-3 (red) (Invitrogen, CA, USA) secondary antibodies, respectively. Nuclei were stained with $5 \mu \mathrm{M}$ DRAQ5 ${ }^{\text {TM }}$ solution (Molecular Probes, OR, USA). (b) Cells were grown on transwell inserts and after reaching a stable TEER, they were transferred into a Cellzscope. Chemical challenge was performed by apical and basolateral addition of hydrogen peroxide. The fluctuation in the TEER measurement at 25 hours resulted from a change in cell culture medium.

To verify the presence of tight junctions in the IHGK cell line, we monitored the TEER in a confluent cell monolayer for a period of 11 days using a Cellzscope (nanoAnalytics, GE). Similar to the values reported by Gröger et al. $(2008)^{291}$, the TEER values remained relatively stable $(80-1000 \mathrm{hm} \mathrm{x} \mathrm{cm}$ ) during the measured period of time. Moreover, we confirmed the expression of two of the major components of the tight junctions (i.e. occludins and zonula occludens-1 (Z0-1) $)^{292,293}$ ) by immunofluorescence (Fig. 2a) and assessed the loss of barrier function upon treatment with different concentrations of hydrogen peroxide (Fig. 2b). Finally, the cells were treated with several microbe-associated molecular patterns (i.e. flagellin, LPS, LTA, Pam2, and Pam3) and pro-inflammatory cytokines (i.e. TNFa and IFNy) at concentrations that we routinely use for other epithelial cell lines (e.g. Ca9-22, H0-1-N-1, and Caco-2). Although increased inflammatory responses are associated with loss of epithelial barrier function ${ }^{294}$, we did not observed a reduction in barrier function with any of the treatments tested. In addition, quantitative PCR analyses and enzyme-linked immunosorbent assays (ELISA) were unable to detect the production of the pro-inflammatory cytokine IL-8. Further analysis revealed that the primary cell stock was infected with mycoplasma, which explains the lack of responsiveness of these cells to the treatments used, and once more highlights the importance of getting cell lines tested for infection on a routine basis. Moreover, it 
exemplifies the current need to establish a suitable oral cell line to study barrier function.

\section{Primary cells and organoids: two steps closer to in vivo studies}

Cell lines provide homogeneous genotypic and phenotypic characteristics that allow high reproducibility of cell-based assays, which is essential in basic research. In addition, cell lines have the ability to proliferate indefinitely, either through spontaneous genetic mutations or artificial modifications (e.g. immortalized cell lines), which make them a convenient and cost-efficient tool to carry out in vitro studies. Nevertheless, due to these characteristics, findings derived from cell lines should be verified using models that can more closely mimic the physiological state in vivo and thereby provide a more biologically relevant readout. Primary cells are isolated directly from human tissue and constitute an alternative model for result-validation as they conserve the characteristics of the original tissue. Recent advances in $3 \mathrm{D}$ culture technology has enabled the differentiation of mammalian stem cells into organoids that reflect the structural and functional properties of organs ${ }^{295}$. Hisha et. al. ${ }^{296}$ established a lingual organoid culture system from adult epithelial stem cells that contained both a stratified squamous epithelial cell layer and the stratum corneum, consisting of flattened dead cell ${ }^{296}$. This technology may open new avenues to study oral wound healing and to explore the effects of particular compounds in subject-specific (patient-derived) organoids.

\section{Understanding the mucosal interactions in the oral ecosystem}

The second section of this discussion focuses on exploring the influence of different components of the oral ecosystem on gingival re-epithelialization using the established high-throughput scratch assay. Using a computational approach, we were able to identify a metabolite signature in saliva that can predict its capacity to induce gingival re-epithelialization in vitro and that relates to the intensity of host-microbe interactions inflammation in vivo that can result in cell damage and/or inflammation (Chapter 5). A similar approach was used to identify oral commensal bacteria that were positive or negatively associated with gingival bleeding scores, which are clinical measurements that are used as a proxy for the inflammatory state of the periodontal tissues. Notably, these associations were also reflected by the capacity of the identified bacteria to stimulate gingival re-epithelialization kinetics in vitro (Chapter 6). The differential effects of selected oral bacteria on re-epithelialization were further studied using a combination of cell-based assays and transcriptomics (Chapter 7). Finally, we performed a screening with a panel of lactic acid bacteria that typically inhabit the oral cavity, leading to the identification of a secreted serine protease of Streptococcus salivarius MS-oral-D6 that promotes gingival re-epithelialization in vitro (Chapter $\mathbf{2}^{155}$ ). The findings of these studies may lead to the development of diagnostic tools to predict disease risk as well as new strategies to promote oral re-epithelialization through administration of nextgeneration probiotics or bioactive compounds that stimulate wound repair.

\section{Metabolite signature, connecting in vitro re-epithelialization kinetics to in vivo oral health status}

Saliva is considered of crucial importance for the maintenance of oral health. Most of this knowledge derives from the observation that persons who lack the ability to produce adequate volumes of saliva experience functional losses that can include mouth dryness (xerostomia), difficulties swallowing, increased susceptibility for bacterial infections, and reduced 
wound healing capacity ${ }^{28,29}$. Saliva contains a myriad of proteins, peptides and metabolites that modulate different aspects of oral health ${ }^{28,297}$. The role of individual saliva constituents (e.g. growth factors, trefoil peptides, secretory leukocyte protease inhibitor (SLPI), histatins) in wound repair has been investigated ${ }^{29}$, but to the best of our knowledge the contribution of the salivary metabolites to this process remained unexplored. In this thesis (Chapter $\mathbf{5}$ ), we used the metabolite profiles of 305 unstimulated saliva samples collected from 61 healthy individuals at 5 timepoints during an experimental gingivitis challenge intervention ${ }^{170}$. Re-epithelialization performance values $\left(\mu_{m}{ }^{*} \mathrm{~A}\right)$ were determined for 61 randomly selected saliva samples, and these values were used to associate them to metabolite patterns measured in the same samples, using elastic net regression ${ }^{178}$ with stability selection ${ }^{179}$. This led to the identification of 10 metabolites (Chapter 5, Table 1) that were collectively associated with the observed re-epithelialization kinetics in vitro. Notably, one of the metabolites in the signature, 0 -sulfo-L-tyrosine was selected in $90 \%$ of the iterations and was assigned the largest weight by elastic net, which may suggest that this metabolite alone could serve as a biomarker to predict saliva-induced re-epithelialization. However, linear regression analyses revealed that the combination of these 10 metabolites into a signature had a significantly higher predictive capacity than 0-sulfo-L-tyrosine, underlining the superior biomarker-value of the signature. Next to 0 -sulfo-L-tyrosine, the signature contains additional metabolites (i.e. host derived lipids, aminoacids and mono-amine derivatives) that could be related to cellular degradation, inflammatory conditions, wound repair, and even chronic periodontitis ${ }^{188,195,197,201,202}$. Thereby, these metabolites appear to reflect increased mucosal damage due to an enhanced host-microbe interplay in the oral cavity. This notion is in agreement with the observation that higher concentrations of these metabolites in saliva samples were associated with enhanced re-epithelialization capacity in vitro, and higher gingival bleeding scores in vivo. Intriguingly, higher intra-individual variation of the metabolite signature over time was positively correlated with an increased response to the gingivitis challenge, reflected by a larger change in gingival bleeding between the baseline and the peak of the challenge. This finding implies that unstable host-microbe interactions and higher variability of the associated metabolite signature may serve as a predictor for elevated risk to develop gingivitis.

This study is constrained to the analysis of the salivary metabolites, and thereby does not take into account that saliva samples may also contain proteins or peptides that have previously been suggested to promote wound repair (e.g. histatins, $\beta$-defensins, cathelicidin). Similarly, growth factors that are released from inflamed tissues and are present in saliva may also contribute to enhanced re-epithelialization. Nevertheless, the study presented implies that the metabolite signature is significantly associated with specific in vitro and in vivo characteristics of the oral mucosa. Unfortunately, since the metabolites used in this study were only quantified as relative concentrations there is no straightforward way to establish their role in re-epithelialization stimulation using mixtures of the purified chemicals that constitute the signature. Further studies would be required to determine the impact of the chemicals in the signature either individually and/or in mixtures on re-epithelialization kinetics. If successful, such studies may open avenues for the incorporation of such chemicals in oral health products aiming to stimulate repair functions of the oral mucosal tissues in patients suffering from oral mucositis or Sjögren syndrome.

\section{Oral commensal bacteria associated with oral health or disease}

The oral cavity provides a warm and moist environment, which in combination with the presence of host-derived nutrients such as saliva proteins, glycoproteins, and gingival crevicular fluid (GCF), support the colonization and growth of many microorganisms ${ }^{28,33}$. Despite the dense colonization of the oral cavity, the resident microbiota normally has a symbiotic 
relationship with the host. However, major ecological pressures (e.g. change in adequate hygiene measures) can disrupt the dynamic equilibrium between the microbiota and the host immune defences, leading to a re-arrangement of the microbial community that can participate in the development of disease ${ }^{33}$.

Active periodontal disease compromises epithelial integrity, favouring bacterial infections that can promote inflammation and tissue destruction $n^{6,11}$. Previous reports have shown that periodontal pathogens such as Porphyromonas gingivalis can efficiently inhibit re-epithelialization in vitro ${ }^{79,80}$, potentially contributing to the disease pathogenesis. In this thesis (Chapter 6), we evaluated the potential role of other oral commensal bacteria in wound repair and their association with the inflammatory state of the periodontal tissues. Elastic net regression ${ }^{178}$ with stability selection ${ }^{179}$ was employed to identify operational taxonomical units (OTUs) positively and negatively associated with gingival bleeding in a large healthy cohort. Representative species of the identified OTUs were screened using the re-epithelialization assay revealing that bacteria negatively associated with gingival bleeding on average stimulated re-epithelialization kinetics to a higher degree as compared to those with a positive association with gingival bleeding. This finding suggests that at least some of the bacteria associated with lower gingival bleeding may support healthy mucosal oral tissues through stimulation of wound repair rather than exacerbation of inflammatory responses that may be elicited by bacteria associated with higher gingival inflammation.

It should be noted that strain specific effects may strongly confound the association of a specific species with health or disease. Therefore, as a way to verify our findings and investigate the role of the selected species during the development of gingivitis, we performed redundancy analyses between the relative abundance of the selected species in two niches (i.e. unstimulated saliva, plaque supragingival) and the gingival bleeding scores recorded for each participant in an experimental gingivitis challenge study $(n=41)$. Notably, despite obvious differences between the studies (e.g. different individuals and genetic makeup, different analyses method), we were able to confirm most of the species (speciescluster) associations with gingival bleeding at the peak of the experimental gingivitis challenge. In particular, higher relative abundances of the representative sequences identified as $A$. oris/viscosus/naes/undii, $R$. dentocariosa, and $V$. dispar had a strong negative association with gingival bleeding, whereas P. catoniae, S. sputigena, and S. anginosus were positively associated with gingival bleeding. One would expect that enhanced gingival bleeding during the experimental gingivitis challenge would be associated with a reduction of the more 'benign' bacteria, possibly in combination with an increasing population of detrimental bacterial species, creating a double-edged sword that may disrupt homeostasis and lead to gingivitis. However, the observations described do not appear to follow such simple ecosystem adaptations, but appear much less predictable. This notion underlines our lack of understanding of ecosystem dynamics and the role of specific microbes in that system. To this end, it is necessary to design studies that aim to identify and understand microbial signatures in a context of ecosystem dynamics as they have a higher potential to predict disease risk than single biomarkers related to health or disease (see Perspective on Oral Health below).

\section{Differential effects of commensal bacteria: benign vs detrimental responses}

The study described in Chapter 6 led to the identification of operational taxonomical units (OTUs) that were associated with in vivo gingival bleeding in two different cohorts. The OTUs assigned to the species groups Actinomyces oris/viscosus/ naes/undii and Veillonella atypica/dispar/parvula were negatively associated with gingival bleeding and subsequent in vitro screenings revealed that representative strains of $A$. oris, $A$. viscosus and $V$. parvula were the strongest and most consistent stimulators of re-epithelialization kinetics among the panel of oral commensal bacteria tested using two dosages (MOI 
100 and 500). In contrast, F. nucleatum subsp. animalis was positively associated with in vivo gingival bleeding and exhibited opposing effects on re-epithelialization in vitro, where an MOI 100 was stimulatory and 500 inhibitory. In Chapter 7 we set out to explore the modulatory responses elicited by these bacteria on gingival re-epithelialization at an MOI 100 using various physiological assays in combination with transcriptome analyses. The transcriptomes of gingival cells were obtained 40 and 120 minutes after introducing a scratch in a confluent cell monolayer. Although the lag time ( $\lambda$ parameter, Chapter $2^{155}$ ) - defined as the time required for the cells to start migrating into the scratch area- was never longer than 20 minutes, the transcriptome responses elicited by our treatments after 40 minutes were quite modest when compared with the moment of introduction of the scratch. A possible explanation for this observation is that some of the key players involved in re-epithelialization (e.g. EGFR, ERK1/2, p38, c-Myc) are activated by protein-phosphorylation, which can occur within a very short timeframe (seconds to minutes) after creation of the scratch. This could imply that the rapid initiation of re-epithelialization is driven by phosphorylation activation of pre-existing cellular machineries involved in migration and proliferation, and therefore not requiring de novo transcription and synthesis of the factors involved. Nonetheless, the transcriptomes obtained after 120 minutes provided a larger difference between the bacterial treatments and the non-treated control (FCS-free DMEM) and therefore, constituted the main focus of our analyses.

Gene set enrichment analyses (GSEA) comparing the cells exposed to the bacteria and the non-treated control revealed that treatment with A. oris, A. viscosus, and V. parvula led to subtle transcriptional responses, supporting the notion that commensal bacteria and the host mucosal responses have co-evolved to achieve a homeostatic balance between tolerance and immunity ${ }^{298}$. Nevertheless, exposure to these bacteria resulted in significant downregulation of the p53 pathway, which could explain the increased cell survival and enhanced re-epithelialization kinetics observed in our in vitro cell-based models. Among the downregulated genes in this pathway were the ones coding for GADD45A, which acts as a 'stress-sensor', and TNFRSF10B that upon activation triggers caspases mediated apoptosis ${ }^{256,257}$. In contrast, $F$. nucleatum subsp. animalis led to significant upregulation of more than 60 gene sets involved in inflammation, apoptosis, adaptive immunity, and detection of extracellular and invading microorganisms, which points towards a potential pathobiont relationship with the host immune responses at the oral mucosal surfaces. In line with this argumentation, exposure of the gingival cells to $F$. nucleatum subsp. animalis led to a significant increased induction of cell death and pro-inflammatory cytokines IL-6 and IL-8. Notably, the central regulatory nodes driving the responses to F. nucleatum subsp. animalis (C-JUN, EST1, EGR1) play important roles in the activation of cell migration and proliferation, which could explain the increased re-epithelialization kinetics observed in our in vitro model. This observation may also provide a link to the association of $F$. nucleatum with oral squamous cell carcinoma $(0 S C C)^{229}$ and its capacity to potentiate intestinal tumorigenesi ${ }^{299,300}$. In addition, F. nucleatum has been shown to adhere to different cell types via its surface adhesin FadA that mediates attachment to E-cadherin $261,262,300-302$. To our knowledge the capacity of $A$. oris, A. viscosus and $V$. parvula to adhere to gingival cell has not been assessed, although it has been described that the $A$. oris fimbrial protein FimA plays a role in adherence to other bacteria as well as eukaryote cells (i.e. red blood cells) ${ }^{303}$. To investigate adherence of the selected bacterial strains to Ca9-22 cells, bacteria were fluorescently labelled with $5 \mu \mathrm{M}$ carboxyfluorescein succinimidyl ester (CFSE) and incubated with Ca9-22 cells for 2 hours at an MOl of 100 and 500. Similar results were obtained with both MOls and when compared to F. nucleatum subsp. animalis, the 'benign' bacteria exhibited considerably lower adherence capacity to the cells, which may be reflected by the subtle transcriptional responses elicited by these bacteria in our analyses as opposed to the extensive responses observed with F. nucleatum subsp. animalis (Figure 3). 

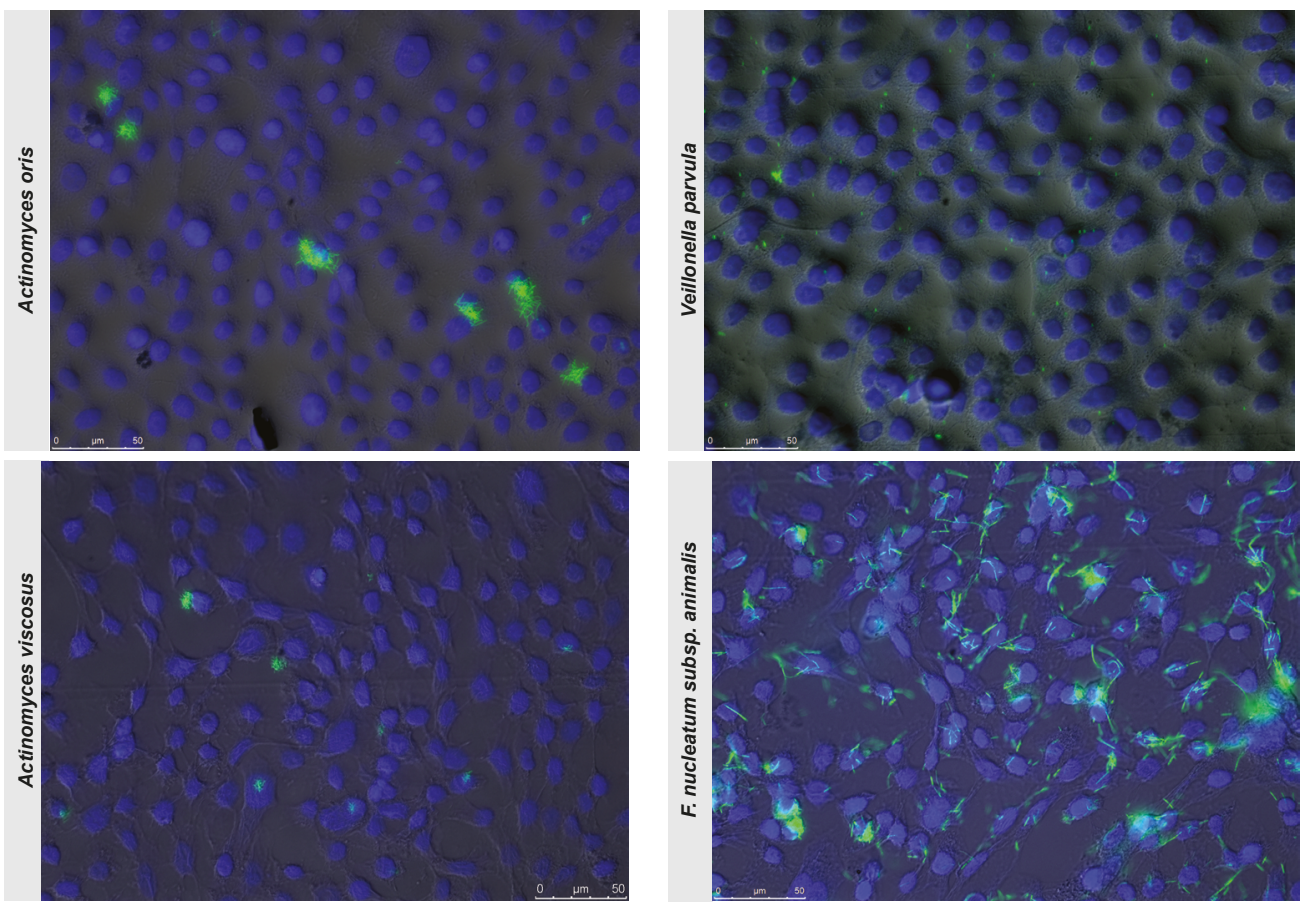

Figure 3. Adhesion of commensal bacteria to gingival cells. Ca9-22 cells were incubated for 2 hours with CFSE-stained bacteria after which cells were washed twice with PBS and fixed using 4\% PFA. Nuclei were stained with $5 \mu \mathrm{MDRAQ5}{ }^{\mathrm{TM}}$ solution. Images shown here were obtained using an MOI of 500 .

\section{Initial steps towards an integrated view of the oral cavity ecosystem}

In Chapter 5, we identified a salivary metabolite signature that was positively associated with increased re-epithelialization capacity, which we proposed to reflect enhanced host-microbe interactions in vivo. To explore this concept from a microbial point of view, we evaluated the relative abundance of the bacteria that were positively and negatively associated with gingival bleeding (Chapter 6 ) in the individuals whose salivary metabolite signatures predicted them to have the highest and lowest re-epithelialization capacity, respectively. Notably, the group of samples that were predicted to have higher re-epithelialization capacity in vitro contained on average higher relative abundances of the bacteria positively associated with gingival bleeding when compared with the group of samples with lower re-epithelialization capacity (Fig. 4a). The average relative abundances of Leptotrichia buccalis, Selenomonas sputigena, and Streptococcus anginosus and intermedius followed that trend of differential abundance. Similarly, zooming-in to the species Fusobacterium nucleatum also revealed its higher average relative abundance in samples predicted to have higher re-epithelialization capacity (Fig. $\mathbf{4 b}$ ). These findings are in agreement with the proposed relation of the salivary metabolite signature with enhanced host-microbe interactions in the oral cavity, and exemplifies the potentially leading role of $F$. nucleatum in this concept. In addition, this also supports the extensive inflammatory responses observed in gingival epithelial cells when they are exposed to this species (Chapter 7). Notably, the cumulative relative abundances of the bacteria that were negatively associated with gingival bleeding was less clearly skewed between the two groups (Fig. $\mathbf{4 c}$ ). However, when examining the relative 
abundances of the representative sequences identified as A. oris/viscosus/naes/undii, we observed a significantly higher average abundance of the species-cluster in the group of samples that were predicted to have lower re-epithelialization capacity $(P=0.03)$ (Fig. $\mathbf{4 d}$ ), and a similar trend was observed for V. parvula (Fig. $\mathbf{4 d}$ ). This observation appears to support the proposed reduced intensity of host-microbe interactions in these individuals, since the responsiveness of epithelial cells to A. oris, A. viscosus or V. parvula was shown to be very mild, supporting the benign role of these microbes (Chapter 7). This apparent congruency of different parameters with the hypothesis that the salivary metabolite signature (Chapter 5) reflects enhanced host-microbe interactions is very intriguing. However, many of the individual observations described above, do not meet the significance threshold, and similar parameter co-appearance determination should be expanded to larger numbers of individuals to corroborate this congruent multi-level signature.

a.

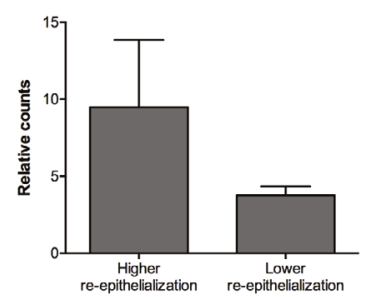

c.

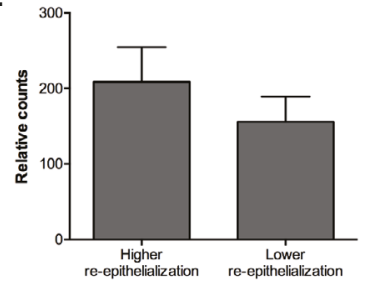

b.

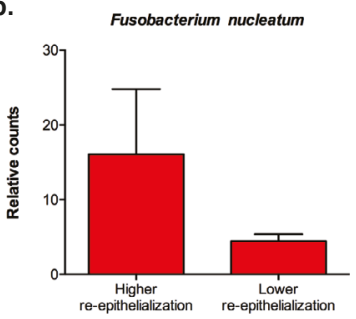

d.

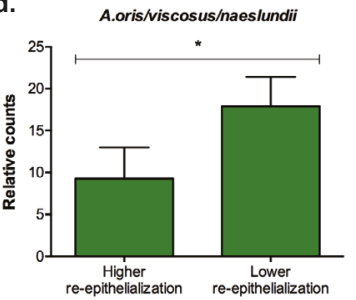

e.

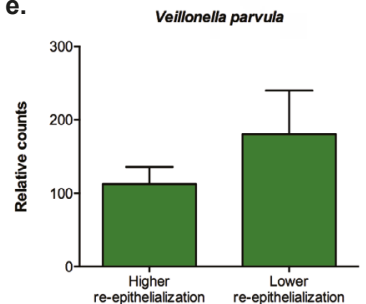

Figure 4. Relative abundance of the commensal species in unstimulated saliva with predicted higher or lower re-epithelialization capacity. The relative abundances of the commensal bacteria were examined in unstimulated saliva samples that were predicted to have the highest and lowest predicted re-epithelialization capacity at the baseline of the experimental gingivitis challenge study (10 saliva samples in each group). (a) Cumulative relative abundances of bacteria positively associated to gingival bleeding. (b) Relative abundances of the representative sequences assigned to $F$. nucleatum. (c) Cumulative relative abundances of bacteria negatively associated to gingival bleeding. (d) Relative abundances of the representative sequences assigned to A. oris/viscosus/naes/undii. (e) Relative abundance of the representative sequence assigned to $V$. parvula. The relative counts are subsampled to equal depth (10,000 reads/sample). Significant differences between groups were assessed by a two-tailed Mann-Whitney $\mathrm{U}$ test $\left({ }^{*}, P\right.$ $<0.05)$.

\section{Lactic acid bacteria and their role in oral health}

Members of the lactic acid bacteria ( $L A B)$ have been widely used as probiotics mostly because of their recognized beneficial impact on gastrointestinal health ${ }^{83,84,86-90}$. Streptococcus and Lactobacillus species are LAB that have a 'negative' reputation in the context of oral health due to their capacity to produce lactic acid through fermentation of carbohydrates that can result in a carious lesion ${ }^{49}$. However, in this thesis (Chapter $\mathbf{2}^{155}$ ) we showed that different lactic acid bacteria species can stimulate gingival re-epithelialization in vitro. Refined experiments using the scratch assay, revealed that 
Streptococcus salivarius strains MS-oral-D6, MS-ileo-F1, and HSISS3 increased re-epithelialization kinetics in a dosedependent manner. Moreover, the spent culture supernatants from these bacteria also had a stimulatory effect on the repair kinetics, indicating the capacity of these bacteria to secrete wound-promoting factors into the media. After removal of small molecules $(<7 \mathrm{kDa}$ ) from the spent media derived from these bacteria only Streptococcus salivarius MS-oral-D6 conditioned medium was able to retain its capacity to promote re-epithelialization. Using in-gel trypsin digestion and LCMS/MS identification of a high molecular weight (MW) secreted protein produced exclusively by this bacterium, we were able to identify a serine protease to be likely involved in the enhanced re-epithelialization observed. The results of this study, illustrate the potential of using microbial components to support oral health through the maintenance of epithelial integrity (see Future Perspectives below).

\section{Future Perspectives}

\section{Next-generation probiotics or bacterial bioactive compounds}

The studies derived from this thesis have led to the identification of oral bacteria that play a role in maintenance of oral health and set the foundations for the development of novel strategies to support oral health through the use of next-generation probiotics or bacterial-bioactive products (Table 2). Nevertheless, follow-up studies are necessary to identify the responsible factors and/or mechanisms by which these bacteria can promote oral health. To this end, protein fractionation methods such as the ones employed in Chapter $\mathbf{2}^{155}$ could provide a way to identify the protein, peptide or (secondary) metabolite responsible for the enhanced re-epithelialization observed with the spent medium derived from $S$. salivarius MS-ileo-F1 and HSISS3. The complete genome sequence of S. salivarius HSISS3 (GCA_000448605.1) is available, which in this case offers the advantage of limiting protein mapping to this particular strain. Moreover, confirmation of the role of eventually identified proteins or peptides on re-epithelialization could be investigated by the construction of knockout (KO) mutations of the encoding gene, which could be greatly facilitated by the peptide pheromone inducible natural competence in S. salivarius ${ }^{304}$. For example, using this system, we could create a KO derivative of the S. salivarius MS-oral-D6 strain that lacks the secreted serine protease and/or the other candidate effector proteins identified in the study (i.e. peptidoglycan hydrolase, peptidase M26, and surface antigen) to assess their specific contribution to the observed re-epithelialization stimulation (Chapter $\mathbf{2}^{155}$ ). A similar strategy could be followed for the identification of the soluble compounds proposed to be secreted by A. oris and V. parvula that act as chemoattractants for gingival cells (Chapter 7). Refined experiments could include microfluidics-based tools, such as the $\mu$-Slide Chemotaxis (ibidi $\mathrm{GmbH}$, Munich, DE) that could be used to monitor cell migration along a stable chemotactic gradient using live-imaging microscopy. The main advantage of this tool in comparison with the Boyden cambers used in Chapter 7 is that it enables the possibility of conducting individual cell trajectory analyses rather than endpoint measurements. Importantly, different established genetic engineering technologies (e.g. Flp-FRT, (re-IoxP, Dre-rox, Tn5, and I-Scel systems) have been used to manipulate the DNA of actinomycetes and may enable the creation of mutants in the Actinomyces species ${ }^{305}$. Following the identification of the stimulating-factors, it is important to assess their conservation among other strains of the same species, which would allow the discrimination of strain- or species-specific effects (Chapter $2^{155}$ ). Moreover, identification of the bioactive compounds produced by these bacteria would allow screening strategies to identify the strain with the highest bioactive production capacity, which could be the most suitable next-generation probiotic. Alternatively, the bioactive production level could be investigated using a variety of growth conditions for these bacteria to tune production 
conditions in such a way that they maximise the bioactive yield in the probiotic product. Finally, bioactive compound identification also allows its application in a more pharmaceutical concept employing only the purified bioactive rather than the producing microbe.

Table 2. Bacteria identified in this thesis that could support oral health.

\begin{tabular}{|c|c|c|c|}
\hline Species & Strain/Isolate & Role in Oral Health & Chapter \\
\hline \multirow{3}{*}{ Streptococcus salivarius } & MS-oral-D6 & \multirow{3}{*}{ Stimulate re-epithelialization kinetics through soluble secreted products } & \multirow{3}{*}{2} \\
\hline & MS-ileo-F1 & & \\
\hline & HSISS3 & & \\
\hline Actinomyces oris & W1053, WVU 474 & \multirow{2}{*}{$\begin{array}{l}\text { Stimulate re-epithelialization kinetics, supports cell survival, and downregulate } \\
\text { stress-associated genes. Proposed to produce soluble secreted compound(s) that } \\
\text { acts as chemoattractant(s) for gingival cells }\end{array}$} & \multirow{3}{*}{6,7} \\
\hline Veillonella parvula & HSIVP1 & & \\
\hline Actinomyces viscosus & Ny 334 & $\begin{array}{l}\text { Stimulate re-epithelialization kinetics, supports cell survival, and downregulate } \\
\text { stress-associated genes }\end{array}$ & \\
\hline
\end{tabular}

\section{Perspective on Oral Health}

The current strategy to support oral health has been focussed on the identification of pathogenic microbial species as well as associated risk parameters that contribute to the initiation or progression of disease. A more effective approach would consist on the development of strategies that aim to actively promote oral health. To this end, it is essential to understand the complex and dynamic network of interactions between different components of the oral ecosystem -including diet, lifestyle, microbiota, saliva, and the host immune system- which determine oral health. The work conducted in this thesis together with other studies ${ }^{211,297,306-308}$ integrated within the framework of the project "Novel Strategies to Promote Oral Health" have started to unravel such interactions in a cohort of healthy individuals and led to the identification of key components associated with oral health, including salivary peptides ${ }^{308}$ and metabolite ${ }^{297}$ as well as microbiota composition patterns ${ }^{211,306,307}$ associated with lower ginigival inflammation (Chapter 6). Moreover, we began to explore the dynamics of the ecosystem upon challenge (e.g. mild gingivitis) and identified signatures in saliva ${ }^{170}$ and microorganisms that could potentially predict/prevent disease development (Chapter $\mathbf{5}$ and $\mathbf{6}$ ). However, we also must conclude that our understanding of the response of the ecosystem to perturbations is still in its infancy. Ecosystem theory has proposed the concept of 'tipping points', which according to Marten Scheffer and co-workers should be loosely defined as "a metaphor for the phenomenon that, beyond a certain threshold, runaway change propels a system to a new state ${ }^{\prime \prime 309}$. This concept may offer possibilities for recognition of early warning signs that precede drastic ecosystem changes ${ }^{310,311}$ associated with the emergence of dysbiosis and disease, although recognizing such warning signs is far from trivial and requires detailed knowledge of the system. Intriguingly, intestinal microbiota members have been suggested as tipping point elements based on their bimodal abundance distribution among individuals and could potentially be used as predictors of ecosystem (in-)stability ${ }^{312}$.

The traditional view of the microbial colonization and biofilm formation in the oral ecosystem has been focussed on the capacity of bacteria to $c 0$-aggregate ${ }^{313}$. However, there are undoubtedly also a variety of metabolic interactions within the oral microbiota that are crucial for microbial interplay within the oral ecosystem. Such metabolic interactions have been used to develop models intending to mathematically describe the syntrophic relations within microbial communities, 


\section{2 | Chapter 8}

including the intestinal microbiota ${ }^{314,315}$. In the oral cavity, the metabolic interaction between lactate producing streptococci and lactate consuming Veillonella species is a clear example of such a syntrophic relation ${ }^{316}$. A more holistic view using multi-omics approaches on in vitro cultured caries associated multispecies biofilm, recently succeeded to describe the complex metabolic interplay that is ongoing in such a biofilm ${ }^{317}$. Studies like these begin to provide insight into the (metabolic) interactions between the bacterial community members of the oral cavity, but it can confidently be assumed that numerous (unknown) interactions between bacteria, host mucosa, saliva and immune system are occurring in the oral ecosystem, which exemplifies its complexity and underlines the challenge to predict its behaviour under dynamic conditions. Holistic and integrated -omics-based approaches (e.g. microbiome, proteome, metabolome, clinical and immune parameters) in the context of health and disease, combined with advanced mathematical modelling and machine learning techniques ${ }^{318}$, may provide the knowledge to better predict system dynamics.

Despite our limited in-depth understanding of the associations uncovered in this thesis, we can utilize these association to propose hypotheses for the mechanisms underlying the interactions between different components of the oral ecosystem (e.g. salivary metabolites, microbes, and mucosal epithelium), and we can also make initial steps towards a more integral view of the interplay in the oral ecosystem. Such findings can be translated into intervention strategies using nextgeneration probiotics and/or their bioactive compounds to promote and sustain oral health. Moreover, these associations may also be used for the development of novel diagnostics that intend to predict disease risk. In particular, saliva appears to provide a non-invasive biofluid that can be employed in diagnostics to assess oral health risk and/or disease, but has also been proposed as a suitable medium for the detection of systemic physiological conditions or pathological states, including cancer, diabetes, and cardiovascular disease ${ }^{319}$. Future research aiming to understand the mechanisms that control the maintenance of health in the oral ecosystem may drive a paradigm shift in dentistry and oral healthcare. 


\section{References}

\section{Summary}

Acknowledgements

About the Author

List of Publications

Overview of Training Activities 



\section{References}

1 (2011). Gendron, R., Grenier, D. \& Maheu-Robert, L.-F. The oral cavity as a reservoir of bacterial pathogens for focal infections. Microbes and Infection 2, $897-906,(2000)$.

3 Koren, 0. et al. Human oral, gut, and plaque microbiota in patients with atherosclerosis. Proceedings of the National Academy of Sciences 108, 4592-4598, (2011).

4 Lalla, E. \& Papapanou, P. N. Diabetes mellitus and periodontitis: a tale of two common interrelated diseases. Nature Reviews Endocrinology 7, 738, (2011). Vos, T. et al. Global, regional, and national incidence, prevalence, and years lived with disability for 310 diseases and injuries, 1990-2015: a systematic analysis for the Global Burden of Disease Study 2015. The Lancet 388, 1545-1602, (2015). Pihlstrom, B. L., Michalowicz, B. S. \& Johnson, N. W. Periodontal diseases. The Lancet 366, 1809-1820, (2005).

Presland, R. \& Jurevic, R. Making sense of the epithelial barrier: what molecular biology and genetics tell us about the functions of oral mucosal and epidermal tissues. Journal of Dental Education 66, 564-574, (2002).

8 Squier, C. A. \& Kremer, M. J. Biology of oral mucosa and esophagus. Journal of the National Cancer Institute. Monographs, 7-15, (2001).

9 Glim, J. E., van Egmond, M., Niessen, F. B., Everts, V. \& Beelen, R. H. J. Detrimental dermal wound healing: What can we learn from the oral mucosa? Wound Repair and Regeneration 21, 648-660, (2013).

10 Presland, R. B. \& Dale, B. A. Epithelial Structural Proteins of the Skin and Oral Cavity: Function in Health and Disease. Critical Reviews in Oral Biology \& Medicine 11, 383-408, (2000).

11 Dale, B. A. Periodontal epithelium: a newly recognized role in health and disease. Periodontology 2000 30, 70-78, (2002).

12 Borradori, L. \& Sonnenberg, A. Structure and Function of Hemidesmosomes: More Than Simple Adhesion Complexes. Journal of Investigative Dermatology 112, 411-418, (1999).

13 Hovav, A. H. Dendritic cells of the oral mucosa. Mucosal Immunol 7, 27-37, (2014).

14 Hancock, R. E. W. \& Chapple, D. S. Peptide Antibiotics. Antimicrobial Agents and Chemotherapy 43, 1317-1323, (1999).

15 Chung, W. 0.\& Dale, B. A. Innate Immune Response of Oral and Foreskin Keratinocytes: Utilization of Different Signaling Pathways by Various Bacterial Species. Infection and Immunity 72, 352-358, (2004).

16 De, Y.et al. Ll-37, the Neutrophil Granule-And Epithelial Cell-Derived Cathelicidin, Utilizes Formyl Peptide Receptor-Like 1 (Fprl1) as a Receptor to Chemoattract Human Peripheral Blood Neutrophils, Monocytes, and T Cells. The Journal of Experimental Medicine 192, 1069, (2000).

17 Ganz, T. Defensins and Host Defense. Science 286, 420, (1999).

18 Oppenheim, J., Biragyn, A., Kwak, L. \& Yang, D. Roles of antimicrobial peptides such as defensins in innate and adaptive immunity. Annals of the Rheumatic Diseases 62, ii17-ii21, (2003).

19 Akira, S. \& Takeda, K. Toll-like receptor signalling. Nat Rev Immuno/ 4, 499-511, (2004).

20 Uriarte, S. M., Edmisson, J. S. \& Jimenez-Flores, E. Human neutrophils and oral microbiota: a constant tug-of-war between a harmonious and a discordant coexistence. Immunological Reviews 273, 282-298, (2016).

21 Hickey, M. J. \& Kubes, P. Intravascular immunity: the host-pathogen encounter in blood vessels. Nature Reviews Immunology 9, 364, (2009).

22 Spielmann, N. \& Wong, D. T. Saliva: diagnostics and therapeutic perspectives. Oral Diseases 17, 345-354, (2011).

23 Dame, Z. T. et al. The human saliva metabolome. Metabolomics 11, 1864-1883, (2015).

24 Oudhoff, M. J. Discovery og the wound-healing capacity of salivary histatins Degree of Doctor thesis, Academic Centre for Dentistry Amsterdam (ACTA), VU University Amsterdam, (2010).

25 Dawes, C. et al. The functions of human saliva: A review sponsored by the World Workshop on Oral Medicine VI. Archives of Oral Biology 60, 863$874,(2015)$.

26 Chiappin, S., Antonelli, G., Gatti, R. \& De Palo, E. F. Saliva specimen: A new laboratory tool for diagnostic and basic investigation. Clinica Chimica Acta 383, 30-40, (2007).

27 Thatcher, B. J., Doherty, A. E., Orvisky, E., Martin, B. M. \& Henkin, R. I. Gustin from Human Parotid Saliva Is Carbonic Anhydrase VI. Biochemical and Biophysical Research Communications 250, 635-641, (1998).

28 Amerongen, A. V. N. \& Veerman, E. C. I. Saliva - the defender of the oral cavity. Oral Diseases 8, 12-22, (2002).

29 Brand, H. S. \& Veerman, E. C. Saliva and wound healing. The Chinese journal of dental research: the official journal of the Scientific Section of the Chinese Stomatological Association (CSA) 16, 7-12, (2013).

30 Dodds, M. W. J., Johnson, D. A. \& Yeh, C.-K. Health benefits of saliva: a review. Journal of Dentistry 33, 223-233, (2005). 
31 Gao, X., Jiang, S., Koh, D. \& Hsu, C.-Y. S. Salivary biomarkers for dental caries. Periodontology 2000 70, 128-141, (2016).

32 Wishart, D. S. et al. HMDB 3.0 - The Human Metabolome Database in 2013. Nucleic Acids Research 41, D801-D807, (2013).

33 Marsh, P. D. \& Devine, D. A. How is the development of dental biofilms influenced by the host? J. Clin. Periodontol. 38, 28-35, (2011).

$34 \mathrm{Xu}, \mathrm{X}$. et al. Oral cavity contains distinct niches with dynamic microbial communities. Environmental Microbiology 17, 699-710, (2015).

35 Slavkin, H. C. Biofilms, Microbial Ecology and Antoni van Leeuwenhoek. The Journal of the American Dental Association 128, 492-495, (1997).

36 Kilian, M. et al. The oral microbiome - An update for oral healthcare professionals. Brit. Dent. J. 221, 657-666, (2016).

37 Keijser, B. J. F. et al. Pyrosequencing analysis of the Oral Microflora of healthy adults. Journal of Dental Research 87, 1016-1020, (2008).

38 Dewhirst, F. E. et al. The Human Oral Microbiome. Journal of Bacteriology 192, 5002-5017, (2010).

39 Zaura, E., Keijser, B. J., Huse, S. M. \& Crielaard, W. Defining the healthy "core microbiome" of oral microbial communities. BMC microbiology 9 , $259,(2009)$.

40 Bik, E. M. et al. Bacterial diversity in the oral cavity of 10 healthy individuals. ISME J 4, 962-974, (2010).

41 Rasiah, I. A., Wong, L., Anderson, S. A. \& Sissons, C. H. Variation in bacterial DGGE patterns from human saliva: over time, between individuals and in corresponding dental plaque microcosms. Archives of Oral Biology 50, 779-787, (2005).

42 Group, T. N. H. W. et al. The NIH Human Microbiome Project. Genome Research 19, 2317-2323, (2009).

43 Costello, E. K. et al. Bacterial Community Variation in Human Body Habitats Across Space and Time. Science 326, 1694-1697, (2009).

44 Li, K., Bihan, M. \& Methé, B. A. Analyses of the Stability and Core Taxonomic Memberships of the Human Microbiome. PLOS ONE 8, e63139, (2013).

45 Zaura, E. \& ten Cate, J. M. Towards Understanding Oral Health. Caries Research 49(suppl 1), 55-61, (2015).

46 Cutler, C. W. \& Jotwani, R. Dendritic Cells at the Oral Mucosal Interface. Journal of Dental Research 85, 678-689, (2006).

47 Zenobia, C. et al. Commensal bacteria-dependent select expression of CXCL2 contributes to periodontal tissue homeostasis. Cellular Microbiology 15, 1419-1426, (2013).

48 Marsh, P. D. Dental plaque as a biofilm and a microbial community - implications for health and disease. BMC Oral Health 6, S14-S14, (2006).

49 Scannapieco, F. A. The oral microbiome: Its role in health and in oral and systemic infections. Clinical Microbiology Newsletter 35, 163-169, (2013).

50 Filoche, S., Wong, L. \& Sissons, C. H. Oral Biofilms: Emerging Concepts in Microbial Ecology. Journal of Dental Research 89, 8-18, (2009).

51 Abariga, S. A. \& Whitcomb, B. W. Periodontitis and gestational diabetes mellitus: A systematic review and meta-analysis of observational studies. BMC Pregnancy and Childbirth 16, (2016).

52 Clothier, B., Stringer, M. \& Jeffcoat, M. K. Periodontal disease and pregnancy outcomes: exposure, risk and intervention. Best Practice \& Research Clinical Obstetrics \& Gynaecology 21, 451-466, (2007).

53 Pussinen, P. J., Laatikainen, T., Alfthan, G., Asikainen, S. \& Jousilahti, P. Periodontitis Is Associated with a Low Concentration of Vitamin C in Plasma. Clinical and Diagnostic Laboratory Immunology 10, 897-902, (2003).

54 Ronderos, M., Jacobs, D. R., Himes, J. H. \& Pihlstrom, B. L. Associations of periodontal disease with femoral bone mineral density and estrogen replacement therapy: cross-sectional evaluation of US adults from NHANES III. Journal of Clinical Periodontology 27, 778-786, (2000).

55 Holmstrup, P. \& Westergaard, J. Periodontal diseases in HIV-infected patients. Journal of Clinical Periodontology 21, 270-280, (1994).

56 Bergström, J. Tobacco smoking and chronic destructive periodontal disease. Odontology 92, 1-8, (2004).

57 Hart, T. C. \& Atkinson, J. C. Mendelian forms of periodontitis. Periodontology 2000 45, 95-112, (2007).

58 Hajishengallis, G., Darveau, R. P. \& Curtis, M. A. The Keystone Pathogen Hypothesis. Nature reviews. Microbiology 10, 717-725, (2012).

59 Mysak, J. et al. Porphyromonas gingivalis: Major Periodontopathic Pathogen Overview. Journal of Immunology Research 2014, 8, (2014).

60 Bodet, C., Chandad, F. \& Grenier, D. Pathogenic potential of Porphyromonas gingivalis, Treponema denticola and Tannerella forsythia, the red bacterial complex associated with periodontitis. Pathologie Biologie 55, 154-162, (2007).

61 Takii, R., Kadowaki, T., Baba, A., Tsukuba, T. \& Yamamoto, K. A Functional Virulence Complex Composed of Gingipains, Adhesins, and Lipopolysaccharide Shows High Affinity to Host Cells and Matrix Proteins and Escapes Recognition by Host Immune Systems. Infection and Immunity 73, 883-893, (2005).

62 Nakayama, M. \& Ohara, N. Molecular mechanisms of Porphyromonas gingivalis-host cell interaction on periodontal diseases. Japanese Dental Science Review 53, 134-140, (2017).

63 Potempa, J. \& Pike, R. N. Corruption of Innate Immunity by Bacterial Proteases. Journal of Innate Immunity 1, 70-87, (2009).

64 Mydel, P.et al. Roles of the Host 0xidative Immune Response and Bacterial Antioxidant Rubrerythrin during Porphyromonas gingivalis Infection. PLOS Pathogens 2, e76, (2006).

65 Boyce, B. F., Yao, Z. \& Xing, L. Osteoclasts have Multiple Roles in Bone in Addition to Bone Resorption. Critical reviews in eukaryotic gene expression 19, 171-180, (2009). 
66 Yost, S., Duran-Pinedo, A. E., Teles, R., Krishnan, K. \& Frias-Lopez, J. Functional signatures of oral dysbiosis during periodontitis progression revealed by microbial metatranscriptome analysis. Genome Medicine 7, 27, (2015).

67 Guo, S. \& DiPietro, L. A. Factors Affecting Wound Healing. Journal of Dental Research 89, 219-229, (2010).

68 Sonnemann, K. J. \& Bement, W. M. Wound Repair: Toward Understanding and Integration of Single-Cell and Multicellular Wound Responses. Annual Review of Cell and Developmental Biology 27, 237-263, (2011).

69 Schäfer, M. \& Werner, S. Transcriptional Control of Wound Repair. Annual Review of Cell and Developmental Biology 23, 69-92, (2007).

70 Cordeiro, J. V. \& Jacinto, A. The role of transcription-independent damage signals in the initiation of epithelial wound healing. 14, 249-262, (2013).

71 Behm, B., Babilas, P., Landthaler, M. \& Schreml, S. Cytokines, chemokines and growth factors in wound healing. Journal of the European Academy of Dermatology and Venereology: JEADV 26, 812-820, (2012).

72 Furie, B. \& Furie, B. C. Thrombus formation in vivo. The Journal of Clinical Investigation 115, 3355-3362, (2005).

73 Werner, S. \& Grose, R. Regulation of wound healing by growth factors and cytokines. Physiological reviews 83, 835-870, (2003).

74 Szpaderska, A. M., Zuckerman, J. D. \& DiPietro, L. A. Differential Injury Responses in Oral Mucosal and Cutaneous Wounds. Journal of Dental Research 82, 621-626, (2003).

75 Oudhoff, M. J. et al. Histatins are the major wound-closure stimulating factors in human saliva as identified in a cell culture assay. FASEB journal : official publication of the Federation of American Societies for Experimental Biology 22, 3805-3812, (2008).

76 Eming, S. A., Krieg, T. \& Davidson, J. M. Inflammation in Wound Repair: Molecular and Cellular Mechanisms. Journal of Investigative Dermatology 127, 514-525.

77 Mallick, S., Benson, R. \& Rath, G. K. Radiation induced oral mucositis: a review of current literature on prevention and management. European Archives of Oto-Rhino-Laryngology 273, 2285-2293, (2016).

78 Laheij, A. M. G. A. et al. Oral bacteria and yeasts in relationship to oral ulcerations in hematopoietic stem cell transplant recipients. Supportive Care in Cancer 20, 3231-3240, (2012).

79 Laheij, A. M., de Soet, J. J., Veerman, E. C., Bolscher, J. G. \& van Loveren, C. The influence of oral bacteria on epithelial cell migration in vitro. Mediators of inflammation 2013, 154532, (2013).

80 Bhattacharya, R. et al. Effect of bacteria on the wound healing behavior of oral epithelial cells. PLOS ONE 9, (2014).

81 FAO/WHO. Guidelines for the evaluation of probiotics in food. (Food and Agricultural Organization of the United Nations and World Health Organization Working Group report., London, Ontario, Canada, 2002).

82 Coconnier, M.-H. et al. Inhibition of adhesion of enteroinvasive pathogens to human intestinal Caco-2 cells by Lactobacillus acidophilus strain LB decreases bacterial invasion. FEMS Microbiology Letters 110, 299-305, (1993).

83 van Baarlen, P., Wells, J. M. \& Kleerebezem, M. Regulation of intestinal homeostasis and immunity with probiotic lactobacilli. Trends Immunol 34, 208-215, (2013).

84 Smelt, M. J. et al. Probiotics Can Generate FoxP3 T-Cell Responses in the Small Intestine and Simultaneously Inducing CD4 and CD8 T Cell Activation in the Large Intestine. PLOS ONE 8, e68952, (2013).

85 de Vrese, M. et al. Probiotics - compensation for lactase insufficiency. The American Journal of Clinical Nutrition 73, 421s-429s, (2001).

86 Anderson, R. C., Cookson, A. L., McNabb, W. C., Kelly, W. J. \& Roy, N. C. Lactobacillus plantarum DSM 2648 is a potential probiotic that enhances intestinal barrier function. FEMS Microbiology Letters 309, 184-192, (2010).

87 Johnson-Henry, K. C., Donato, K. A., Shen-Tu, G., Gordanpour, M. \& Sherman, P. M. Lactobacillus rhamnosus Strain GG Prevents Enterohemorrhagic Escherichia coli 0157:H7-Induced Changes in Epithelial Barrier Function. Infection and Immunity 76, 1340-1348, (2008).

88 Karczewski, J. et al. Regulation of human epithelial tight junction proteins by Lactobacillus plantarum in vivo and protective effects on the epithelial barrier. American Journal of Physiology - Gastrointestinal and Liver Physiology 298, G851, (2010).

89 Yan, F. et al. Soluble Proteins Produced by Probiotic Bacteria Regulate Intestinal Epithelial Cell Survival and Growth. Gastroenterology 132, 562575, (2007).

90 van Baarlen, P. et al. Human mucosal in vivo transcriptome responses to three lactobacilli indicate how probiotics may modulate human cellular pathways. Proceedings of the National Academy of Sciences 108, 4562-4569, (2011).

91 Mohammedsaeed, W., Cruickshank, S., McBain, A. J. \& 0'Neill, C. A. Lactobacillus rhamnosus GG Lysate Increases Re-Epithelialization of Keratinocyte Scratch Assays by Promoting Migration. Scientific Reports 5, 16147, (2015).

92 Reid, G. Probiotic agents to protect the urogenital tract against infection. The American Journal of Clinical Nutrition 73, 437s-443s, (2001).

93 Reid, G. \& Bruce, A. W. Probiotics to prevent urinary tract infections: the rationale and evidence. World Journal of Urology 24, 28-32, (2006).

94 Gungor, 0. E., Kirzioglu, Z. \& Kivanc, M. Probiotics: Can they be used to improve oral health? Beneficial Microbes 6, 647-656, (2015).

95 Snel, J. et al. Competitive selection of lactic acid bacteria that persist in the human oral cavity. Applied and environmental microbiology 77, 8445-

8450, (2011). 
96 Piwat, S., Sophatha, B. \& Teanpaisan, R. An assessment of adhesion, aggregation and surface charges of Lactobacillus strains derived from the human oral cavity. Letters in Applied Microbiology 61, 98-105, (2015).

97 Sookkhee, S., Chulasiri, M. \& Prachyabrued, W. Lactic acid bacteria from healthy oral cavity of Thai volunteers: inhibition of oral pathogens. Journal of Applied Microbiology 90, 172-179, (2001).

98 BuTerai, T. et al. Screening of Probiotic Candidates in Human Oral Bacteria for the Prevention of Dental Disease. PLoS ONE 10, e0128657, (2015).

99 Burton, J. P., Chilcott, C. N., Moore, C. J., Speiser, G. \& Tagg, J. R. A preliminary study of the effect of probiotic Streptococcus salivarius K12 on oral malodour parameters. Journal of Applied Microbiology 100, 754-764, (2006).

100 Cosseau, C. et al. The commensal Streptococcus salivarius K12 downregulates the innate immune responses of human epithelial cells and promotes host-microbe homeostasis. Infection and immunity 76, 4163-4175, (2008).

101 Tanner, A. \& Maiden, M. F. J. Gingivitis and the initial periodontal lesion. Microbial Ecology in Health and Disease 9, 359-365, (1996).

102 Köll-Klais, P., Mändar, R., Leibur, E. \& Mikelsaar, M. Oral microbial ecology in chronic periodontitis and periodontal health. Microbial Ecology in Health and Disease 17, 146-155, (2005).

103 Liang, C. C., Park, A. Y. \& Guan, J. L. In vitro scratch assay: a convenient and inexpensive method for analysis of cell migration in vitro. Nature protocols 2, 329-333, (2007).

104 Hulkower, K. I. \& Herber, R. L. Cell Migration and Invasion Assays as Tools for Drug Discovery. Pharmaceutics 3, 107-124, (2011).

105 Zwietering, M. H., Jongenburger, I., Rombouts, F. M. \& van 't Riet, K. Modeling of the Bacterial Growth Curve. Applied and Environmental Microbiology 56, 1875-1881, (1990).

106 Zhang, J.-H., Chung, T. D. Y. \& Oldenburg, K. R. A Simple Statistical Parameter for Use in Evaluation and Validation of High Throughput Screening Assays. Journal of Biomolecular Screening 4, 67-73, (1999).

107 van den Bogert, B. et al. Diversity of human small intestinal Streptococcus and Veillonella populations. FEMS Microbiology Ecology 85, 376-388, (2013).

108 Lam, E. K. et al. Probiotic Lactobacillus rhamnosus GG enhances gastric ulcer healing in rats. European journal of pharmacology 565, 171-179, (2007).

109 Bowden, G. H. W. The microbial ecology of dental caries. Microbial Ecology in Health and Disease 12, 138-148, (2000).

110 Piwat, S., Teanpaisan, R., Thitasomakul, S., Thearmontree, A. \& Dahlén, G. Lactobacillus species and genotypes associated with dental caries in Thai preschool children. Molecular Oral Microbiology 25, 157-164, (2010).

111 Söderling, E. M., Marttinen, A. M. \& Haukioja, A. L. Probiotic Lactobacilli Interfere with Streptococcus mutans Biofilm Formation In Vitro. Current Microbiology 62, 618-622, (2011).

112 Nikawa, H. et al. Lactobacillus reuteri in bovine milk fermented decreases the oral carriage of mutans streptococci. International Journal of Food Microbiology 95, 219-223, (2004).

113 Çaglar, E., Kavaloglu Cildir, S., Ergeneli, S., Sandalli, N. \& Twetman, S. Salivary mutans streptococci and lactobacilli levels after ingestion of the probiotic bacterium Lactobacillus reuteri ATCC 55730 by straws or tablets. Acta Odontologica Scandinavica 64, 314-318, (2006).

114 Çaglar, E. et al. Effect of chewing gums containing xylitol or probiotic bacteria on salivary mutans streptococci and lactobacilli. Clinical Oral Investigations 11, 425-429, (2007).

115 Twetman, S. et al. Short-term effect of chewing gums containing probiotic Lactobacillus reuteri on the levels of inflammatory mediators in gingival crevicular fluid. Acta Odontologica Scandinavica 67, 19-24, (2009).

116 Shimauchi, H. et al. Improvement of periodontal condition by probiotics with Lactobacillus salivarius WB21: a randomized, double-blind, placebo-controlled study. Journal of Clinical Periodontology 35, 897-905, (2008).

$117 \mathrm{Na0}$, S. et al. Effects of oil drops containing Lactobacillus salivarius WB21 on periodontal health and oral microbiota producing volatile sulfur compounds. Journal of Breath Research 6, 017106, (2012).

118 Yarrow, J. C., Perlman, Z. E., Westwood, N. J. \& Mitchison, T. J. A high-throughput cell migration assay using scratch wound healing, a comparison of image-based readout methods. BMC biotechnology 4, 21, (2004).

119 Yue, P. Y. K., Leung, E. P. Y., Mak, N. K. \& Wong, R. N. S. A Simplified Method for Quantifying Cell Migration/Wound Healing in 96-Well Plates. Journal of Biomolecular Screening 15, 427-433, (2010).

120 Geback, T., Schulz, M. M., Koumoutsakos, P. \& Detmar, M. TScratch: a novel and simple software tool for automated analysis of monolayer wound healing assays. BioTechniques 46, 265-274, (2009).

121 Yan, F. et al. A Lactobacillus rhamnosus GG-derived Soluble Protein, p40, Stimulates Ligand Release from Intestinal Epithelial Cells to Transactivate Epidermal Growth Factor Receptor. The Journal of Biological Chemistry 288, 30742-30751, (2013).

122 Pearce, C. et al. Identification of pioneer viridans streptococci in the oral cavity of human neonates. Journal of Medical Microbiology 42, 67-72, (1995). 
123 van den Bogert, B., de Vos, W. M., Zoetendal, E. G. \& Kleerebezem, M. Microarray Analysis and Barcoded Pyrosequencing Provide Consistent Microbial Profiles Depending on the Source of Human Intestinal Samples. Applied and Environmental Microbiology 77, 2071-2080, (2011).

124 Delorme, C., Abraham, A.-L., Renault, P. \& Guédon, E. Genomics of Streptococcus salivarius, a major human commensal. Infection, Genetics and Evolution 33, 381-392, (2015).

125 Trabold, 0. et al. Lactate and oxygen constitute a fundamental regulatory mechanism in wound healing. Wound Repair and Regeneration 11, 504-509, (2003).

126 Ghani, Q. P., Wagner, S., Becker, H. D., Hunt, T. K. \& Hussain, M. Z. Regulatory Role of Lactate in Wound Repair. Methods in Enzymology Volume 381, 565-575, (2004).

127 Landén, N. X., Li, D. \& Ståhle, M. Transition from inflammation to proliferation: a critical step during wound healing. Cellular and Molecular Life Sciences 73, 3861-3885, (2016).

128 Eming, S. A., Krieg, T. \& Davidson, J. M. Inflammation in Wound Repair: Molecular and Cellular Mechanisms. Journal of Investigative Dermatology 127, 514-525, (2007).

129 Koch, A. E. et al. Interleukin-8 as a macrophage-derived mediator of angiogenesis. Science 258, 1798-1798, (1992).

130 Siezen, R. J. \& Leunissen, J. A. Subtilases: the superfamily of subtilisin-like serine proteases. Protein Science : A Publication of the Protein Society 6, 501-523, (1997).

131 International consensus. The role of proteases in wound diagnostics. An expert working group review. London: Wounds International. (2011).

132 Kleerebezem, M. et al. Complete genome sequence of Lactobacillus plantarum WCFS1. Proceedings of the National Academy of Sciences 100, 1990-1995, (2003).

133 Gorbach, S. L. \& Goldin, B. R. Lactobacillus strains and methods of selection. U.S. Patent No. 4,839,281. 13 Jun. (1989).

134 Hayward, A. C. \& Davis, G. H. G. The isolation and classification of Lactobacillus strains from Italian saliva samples. Br. Dent. J. 101, 43-46, (1956).

135 Van den Bogert, B. et al. Comparative Genomics Analysis of Streptococcus Isolates from the Human Small Intestine Reveals their Adaptation to a Highly Dynamic Ecosystem. PLOS ONE 8, e83418, (2013).

136 De Ryck, T. et al. Microbial inhibition of oral epithelial wound recovery: potential role for quorum sensing molecules? AMB Express 5, 27, (2015).

137 Laemmli sample buffer (2X). Cold Spring Harbor Protocols 2006, pdb.rec10424, (2006).

138 Huang, C., Jacobson, K. \& Schaller, M. D. MAP kinases and cell migration. Journal of Cell Science 117, 4619-4628, (2004).

139 Sharma, G.-D., He, J. \& Bazan, H. E. P. p38 and ERK1/2 Coordinate Cellular Migration and Proliferation in Epithelial Wound Healing: Evidence of Cross-talk Activation Between MAP Kinase Cascades. Journal of Biological Chemistry 278, 21989-21997, (2003).

140 Mebratu, Y. \& Tesfaigzi, Y. How ERK1/2 activation controls cell proliferation and cell death: Is subcellular localization the answer? Cell Cycle 8, 1168-1175, (2009).

141 R: A Language and Environment for Statistical Computing. (R Foundation for Statistical Computing, Vienna, Austria, 2011).

142 minpack.Im: R Interface to the Levenberg-Marquardt Nonlinear Least-squares Algorithm Found in MINPACK, Plus Support for Bounds. R package version 1.2-1. https://cran.r-project.org/package=minpack.Im (2016).

143 Gavin, H. The Levenberg-Marquardt method for nonlinear least squares curve-fitting problems. (Department of Civil and Environmental Engineering, Duke University, 2011).

144 Tomita, S. et al. Characterization of the transcriptional regulation of the tarlJKL locus involved in ribitol-containing wall teichoic acid biosynthesis in Lactobacillus plantarum. Microbiology 162, 420-432, (2016).

145 Ridley, A. J. et al. Cell Migration: Integrating Signals from Front to Back. Science 302, 1704-1709, (2003).

146 Friedl, P. \& Gilmour, D. Collective cell migration in morphogenesis, regeneration and cancer. Nat Rev Mol Cell Biol 10, 445-457, (2009).

147 Oudhoff, M. J. et al. Histatins enhance wound closure with oral and non-oral cells. Journal of Dental Research 88, 846-850, (2009).

148 Zordan, M. D., Mill, C. P., Riese, D. J., 2nd \& Leary, J. F. A high throughput, interactive imaging, bright-field wound healing assay. Cytometry. Part A : the journal of the International Society for Analytical Cytology 79, 227-232, (2011).

149 Simpson, K. J. et al. Identification of genes that regulate epithelial cell migration using an siRNA screening approach. Nat Cell Biol 10, 1027-1038, (2008).

150 Lamprecht, M. R., Sabatini, D. M. \& Carpenter, A. E. CellProfiler ${ }^{\mathrm{TM}}$ : Free, versatile software for automated biological image analysis. BioTechniques 42,71-75, (2007).

151 Schindelin, J., Rueden, C. T., Hiner, M. C. \& Eliceiri, K. W. The ImageJ ecosystem: An open platform for biomedical image analysis. Molecular Reproduction and Development 82, 518-529, (2015).

152 A Flow Cytometry Analysis Environment for Image Cytometry Data. Available from: https://www.denovosoftware.com/site/Image-0verview. shtml v. 6.

153 WimScratch: Wound Healing Assay Image Analysis Solution. Availabe from: https://www.wimasis.com/en/products/9/WimScratch v. 4.0 (2016). 
154 Goecks, J., Nekrutenko, A. \& Taylor, J. Galaxy: a comprehensive approach for supporting accessible, reproducible, and transparent computational research in the life sciences. Genome Biology 11, R86-R86, (2010).

155 Fernandez-Gutierrez, M. M. et al. Streptococcus salivarius MS-oral-D6 promotes gingival re-epithelialization in vitro through a secreted serine protease. Scientific Reports 7, 11100, (2017).

156 Lindblad, J. \& Bengtsson, E. in Proceedings of the 12th Scandinavian Conference on Image Analysis (SCIA). (ed I. Austvoll) 264-271.

157 Otsu, N. A Threshold Selection Method from Gray-Level Histograms. IEEE Transactions on Systems, Man, and Cybernetics 9, 62-66, (1979).

158 Bürk, R. R. A Factor from a Transformed Cell Line That Affects Cell Migration. Proceedings of the National Academy of Sciences 70, 369-372, (1973).

159 Coomber, B. L. \& Gotlieb, A. I. In vitro endothelial wound repair. Interaction of cell migration and proliferation. Arteriosclerosis (Dallas, Tex.) 10, 215-222, (1990).

160 Riahi, R., Yang, Y., Zhang, D. D. \& Wong, P. K. Advances in Wound-Healing Assays for Probing Collective Cell Migration. Journal of Laboratory Automation 17, 59-65, (2012).

161 Fernandez-Gutierrez, M. M., van Zessen, D. B. H., van Baarlen, P., Kleerebezem, M. \& Stubbs, A. P. KREAP: An automated Galaxy Platform to Quantify Re-epithelialization Kinetics. GigaScience, (2017).

162 Ebner, R. \& Derynck, R. Epidermal growth factor and transforming growth factor-alpha: differential intracellular routing and processing of ligand-receptor complexes. Cell Regulation 2, 599-612, (1991).

163 Zhang, W. \& Liu, H. T. MAPK signal pathways in the regulation of cell proliferation in mammalian cells. Cell Res 12, 9-18, (2002).

164 Svensjo, T., Pomahac, B., Yao, F., Slama, J. \& Eriksson, E. Accelerated healing of full-thickness skin wounds in a wet environment. Plastic and Reconstructive Surgery 106, 602-612, (2000).

165 Zelles, T., Purushotham, K. R., Macauley, S. P., Oxford, G. E. \& Humphreys-Beher, M. G. Concise Review: Saliva and Growth Factors: The Fountain of Youth Resides in Us All. J. Dent. Res. 74, 1826-1832, (1995).

166 Mogi, M., Harada, M., Inagaki, H., Minami, M. \& Kojima, K. Transforming growth factor-a in human submandibular gland and saliva. Journal of Immunoassay 16, 379-394, (1995).

167 Keswani, S. G. et al. Role of salivary vascular endothelial growth factor (VEGF) in palatal mucosal wound healing. Wound Repair and Regeneration 21, 554-562, (2013).

168 Niyonsaba, F. et al. Antimicrobial Peptides Human Beta-Defensins Stimulate Epidermal Keratinocyte Migration, Proliferation and Production of Proinflammatory Cytokines and Chemokines. Journal of Investigative Dermatology 127, 594-604.

169 von Haussen, J. et al. The host defence peptide LL-37/hCAP-18 is a growth factor for lung cancer cells. Lung Cancer 59, 12-23.

170 Prodan, A. et al. Effect of experimental gingivitis induction and erythritol on the salivary metabolome and functional biochemistry of systemically healthy young adults. Metabolomics 12, 147, (2016).

171 Runnel, R. et al. Effect of three-year consumption of erythritol, xylitol and sorbitol candies on various plaque and salivary caries-related variables. Journal of Dentistry 41, 1236-1244, (2013).

172 Mäkinen, K. K. et al. Similarity of the Effects of Erythritol and Xylitol on SomeRisk Factors of Dental Caries. Caries Research 39, 207-215, (2005).

173 Honkala, S. et al. Effect of Erythritol and Xylitol on Dental Caries Prevention in Children. Caries Research 48, 482-490, (2014).

174 Löe, H., Theilade, E. \& Jensen, S. B. Experimental Gingivitis in Man. Journal of Periodontology 36, 177-187, (1965).

175 World Medical, A. World medical association declaration of helsinki: Ethical principles for medical research involving human subjects. JAMA 310, 2191-2194, (2013).

176 Prodan, A. et al. Interindividual variation, correlations, and sex-related differences in the salivary biochemistry of young healthy adults. European Journal of Oral Sciences 123, 149-157, (2015).

177 Oliveira, S. C. et al. Correlations between two different methods to score bleeding and the relationship with plaque in systemically healthy young adults. Journal of Clinical Periodontology 42, 908-913, (2015).

178 Zou, H. \& Hastie, T. Regularization and variable selection via the elastic net. Journal of the Royal Statistical Society: Series B (Statistical Methodology) 67, 301-320, (2005).

179 Meinshausen, N. \& Bühlmann, P. Stability selection. Journal of the Royal Statistical Society: Series B (Statistical Methodology) 72, 417-473, (2010).

180 Pedregosa, F. et al. Scikit-learn: Machine Learning in Python. Journal of Machine Learning Research 12, 2825-2830, (2011).

181 Warnes, G. et al. gplots: Various R programming tools for plotting data. Vol. v 3.0.1 (2016).

182 Hyndman, R. J. \& Koehler, A. B. Another look at measures of forecast accuracy. International Journal of Forecasting 22, 679-688, (2006).

183 Goodier, J. The Cambridge Dictionary of Statistics (4th edition). Reference Reviews 25, 37-38, (2011).

184 Löe, H. The Gingival Index, the Plaque Index and the Retention Index Systems. Journal of Periodontology 38, 610-616, (1967).

185 Milanowski, M., Pomastowski, P., Ligor, T. \& Buszewski, B. Saliva-Volatile Biomarkers and Profiles. Critical Reviews in Analytical Chemistry 47, 251-266, (2017). 
Zhang, A., Sun, H. \& Wang, X. Saliva Metabolomics Opens Door to Biomarker Discovery, Disease Diagnosis, and Treatment. Applied Biochemistry and Biotechnology 168, 1718-1727, (2012).

Arakaki, A. K., Skolnick, J. \& McDonald, J. F. Marker metabolites can be therapeutic targets as well. Nature 456, 443-443, (2008).

Barnes, V. M. et al. Metabolomics Reveals Elevated Macromolecular Degradation in Periodontal Disease. Journal of Dental Research 90, 12931297, (2011).

Sugimoto, M., Wong, D. T., Hirayama, A., Soga, T. \& Tomita, M. Capillary electrophoresis mass spectrometry-based saliva metabolomics identified oral, breast and pancreatic cancer-specific profiles. Metabolomics 6, 78-95, (2010).

Kaufman, E. \& Lamster, I. B. The Diagnostic Applications of Saliva— A Review. Critical Reviews in Oral Biology \& Medicine 13, 197-212, (2002).

Braverman, N. E. \& Moser, A. B. Functions of plasmalogen lipids in health and disease. Biochimica et Biophysica Acta (BBA) - Molecular Basis of Disease 1822, 1442-1452, (2012).

Nagan, N. \& Zoeller, R. A. Plasmalogens: Biosynthesis and functions. Progress in Lipid Research 40, 199-229, (2001).

Broniec, A. et al. Interactions of plasmalogens and their diacyl analogs with singlet oxygen in selected model systems. Free Radical Biology and Medicine 50, 892-898, (2011).

Sindelar, P. J., Guan, Z., Dallner, G. \& Ernster, L. The protective role of plasmalogens in iron-induced lipid peroxidation. Free Radical Biology and Medicine 26, 318-324, (1999).

Kosinska, M. K. et al. Articular Joint Lubricants during Osteoarthritis and Rheumatoid Arthritis Display Altered Levels and Molecular Species. PLoS ONE 10, e0125192, (2015).

Wu, C.-L., Kimmerling, K. A., Little, D. \& Guilak, F. Serum and synovial fluid lipidomic profiles predict obesity-associated osteoarthritis, synovitis, and wound repair. Scientific Reports 7, 44315, (2017).

Vaughan, E. M. et al. Lipid domain-dependent regulation of single-cell wound repair. Molecular Biology of the Cell 25, 1867-1876, (2014).

Richter, K. et al. Phosphocholine - an agonist of metabotropic but not of ionotropic functions of a9-containing nicotinic acetylcholine receptors. 6, 28660, (2016).

Kiss, Z. \& Chung, T. Choline Phosphate and Phorbol Ester Potentiate the Mitogenic Effect of Insulin by Competitive Mechanisms in NIH 3T3 Fibroblasts. Biochemical and Biophysical Research Communications 218, 505-509, (1996).

Tomono, M., Crilly, K. S. \& Kiss, Z. Synergistic Potentiating Effects of Choline Phosphate and Ethanolamine on Insulin-Induced DNA Synthesis in NIH 3 T3 Fibroblasts. Biochemical and Biophysical Research Communications 213, 980-985, (1995).

Callery, P. S. \& Geelhaar, L. A. Biosynthesis of 5-Aminopentanoic Acid and 2-Piperidone from Cadaverine and 1-Piperideine in Mouse. Journal of Neurochemistry 43, 1631-1634, (1984).

Syrjänen, S., Piironen, P. \& Markkanen, H. Free amino-acid content of wax-stimulated human whole saliva as related to periodontal disease. Archives of Oral Biology 32, 607-610, (1987).

Kanan, Y. \& Al-Ubaidi, M. Tyrosine 0 Sulfation: An Overview. JSM Biotechnology and Biomedical Engineering 1, (2013).

Kehoe, J. W. \& Bertozzi, C. R. Tyrosine sulfation: a modulator of extracellular protein-protein interactions. Chemistry \& Biology 7, R57-R61, (2000).

Yang, Y.-S. et al. Tyrosine Sulfation as a Protein Post-Translational Modification. Molecules 20, 2138, (2015). Brito-Zerón, P. et al. Sjögren syndrome. 2, 16047, (2016).

Darveau, R. P., Tanner, A. \& Page, R. C. The microbial challenge in periodontitis. Periodontology 2000 14, 12-32, (1997).

Hasegawa, Y. et al. Gingival epithelial cell transcriptional responses to commensal and opportunistic oral microbial species. Infect. Immun. 75, 2540-2547, (2007).

Kesic, L., Milasin, J., Igic, M. \& Obradovic, R. Microbial Etiology of Periodontal Disease - Mini Review. Medicine and Biology 15, 1-6, (2008).

Cochran, D. L. Inflammation and bone loss in periodontal disease. J. Periodontol. 79, 1569-1576, (2008).

Zaura, E. et al. On the ecosystemic network of saliva in healthy young adults. ISMEJ, (2017).

Fernandez-Gutierrez, M. M. et al. Salivary metabolite signature predicts in vitro re-epithelialization kinetics and is associated with gingival bleeding in human volunteers. Manuscript in preparation. (2017).

213 Chen, T. et al. The Human Oral Microbiome Database: a web accessible resource for investigating oral microbe taxonomic and genomic information. Database 2010, (2010).

4 Altschul, S. F., Gish, W., Miller, W., Myers, E. W. \& Lipman, D. J. Basic local alignment search tool. Journal of Molecular Biology 215, 403-410, (1990).

5 Ter Braak, C. J. F. Canonical Correspondence Analysis: A New Eigenvector Technique for Multivariate Direct Gradient Analysis. Ecology 67, 11671179, (1986).

6 Ter Braak CJF, Smilauer P. Canoco reference manual and user's guide: software for ordination. Ithaca, USA: Microcomputer Power; 2012. p. 496. 
217 Zaura, E., Nicu, E. A., Krom, B. P. \& Keijser, B. J. F. Acquiring and maintaining a normal oral microbiome: current perspective. Frontiers in Cellular and Infection Microbiology 4, (2014).

218 Marsh, P. D. Dental diseases--are these examples of ecological catastrophes? Int J Dent Hyg 4 Suppl 1, 3-10; discussion 50-52, (2006).

219 Marsh, P. D. Dental plaque: biological significance of a biofilm and community life-style. Journal of Clinical Periodontology 32, 7-15, (2005).

220 Berezow, A. B. \& Darveau, R. P. Microbial shift and periodontitis. Periodontology 2000 55, 36-47, (2011).

221 Pihlstrom, B. L., Michalowicz, B. S. \& Johnson, N. W. Periodontal diseases. The Lancet 366, 1809-1820.

222 Moore, W. E. C. \& Moore, L. V. H. The bacteria of periodontal diseases. Periodontology 2000 5, 66-77, (1994).

223 Teles, R. et al. Relationships Among Gingival Crevicular Fluid Biomarkers, Clinical Parameters of Periodontal Disease, and the Subgingival Microbiota. Journal of Periodontology 81, 89-98, (2009).

224 Kumar, P. S. et al. Changes in Periodontal Health Status Are Associated with Bacterial Community Shifts as Assessed by Quantitative 16S Cloning and Sequencing. Journal of Clinical Microbiology 44, 3665-3673, (2006).

225 Meuric, V. et al. Signature of microbial dysbiosis in periodontitis. Applied and Environmental Microbiology 83, (2017).

226 Kirst, M. E. et al. Dysbiosis and Alterations in Predicted Functions of the Subgingival Microbiome in Chronic Periodontitis. Applied and Environmental Microbiology 81, 783-793, (2015).

227 Slots, J., MÖEnbo, D., Langebaek, J. \& Frandsen, A. Microbiota of gingivitis in man. European Journal of Oral Sciences 86, 174-181, (1978).

228 Moore, W. E. et al. Bacteriology of experimental gingivitis in young adult humans. Infect. Immun. 38, 651-667, (1982).

229 Al-Hebshi, N. N. et al. Inflammatory bacteriome featuring Fusobacterium nucleatum and Pseudomonas aeruginosa identified in association with oral squamous cell carcinoma. Scientific Reports 7, (2017).

230 Morita, E. et al. Different frequencies of Streptococcus anginosus infection in oral cancer and esophageal cancer. Cancer Science 94, 492-496, (2003).

231 Sasaki, H. et al. Presence of Streptococcus anginosus DNA in Esophageal Cancer, Dysplasia of Esophagus, and Gastric Cancer. Cancer Research 58, 2991, (1998).

232 Tateda, M. et al. Streptococcus anginosus in head and neck squamous cell carcinoma: implication in carcinogenesis. International Journal of Molecular Medicine 6, 699-703, (2000).

233 Takeshita, T. et al. Distinct composition of the oral indigenous microbiota in South Korean and Japanese adults. Scientific Reports 4, 6990, (2014).

234 Hajishengallis, G. Porphyromonas gingivalis-host interactions: open war or intelligent guerilla tactics? Microbes and Infection 11, 637-645, (2009).

235 Mardis, E. R. Next-Generation Sequencing Platforms. Annual Review of Analytical Chemistry 6, 287-303, (2013).

236 Fernandez-Gutierrez, M. M. et al. Identification of Oral Commensal Bacteria Related to Gingival Bleeding and Re-Epithelialization with DiseaseRisk Signature Potential. Manuscript in preparation. (2017).

237 Jorissen, R. N. et al. Epidermal growth factor receptor: mechanisms of activation and signalling. Experimental Cell Research 284, 31-53, (2003).

238 Boyden, S. The Chemotactic Effect of Mixtures of Antibody and Antigen on Polymorphonuclear Leucocytes. The Journal of Experimental Medicine 115, 453-466, (1962).

239 Vermes, I., Haanen, C., Steffens-Nakken, H. \& Reutellingsperger, C. A novel assay for apoptosis Flow cytometric detection of phosphatidylserine expression on early apoptotic cells using fluorescein labelled Annexin V. Journal of Immunological Methods 184, 39-51, (1995).

240 Lin, K. et al. in Journal of Integrative Bioinformatics Vol. 859 (2011).

241 Heber, S. \& Sick, B. Quality Assessment of Affymetrix GeneChip Data. OMICS: A Journal of Integrative Biology 10, 358-368, (2006).

242 Dai, M. et al. Evolving gene/transcript definitions significantly alter the interpretation of GeneChip data. Nucleic Acids Research 33, e175-e175, (2005).

243 Bolstad, B. M., Irizarry, R. A., Åstrand, M. \& Speed, T. P. A comparison of normalization methods for high density oligonucleotide array data based on variance and bias. Bioinformatics 19, 185-193, (2003).

244 Smyth, G. K. Linear Models and Empirical Bayes Methods for Assessing Differential Expression in Microarray Experiments. Statistical Applications in Genetics and Molecular Biology 3, (2004).

245 Howe, E. A., Sinha, R., Schlauch, D. \& Quackenbush, J. RNA-Seq analysis in MeV. Bioinformatics 27, 3209-3210, (2011).

246 Subramanian, A. et al. Gene set enrichment analysis: A knowledge-based approach for interpreting genome-wide expression profiles. Proceedings of the National Academy of Sciences 102, 15545-15550, (2005).

247 Shannon, P. et al. Cytoscape: A Software Environment for Integrated Models of Biomolecular Interaction Networks. Genome Research 13, 24982504, (2003).

248 Martin, A. et al. BisoGenet: a new tool for gene network building, visualization and analysis. BMC Bioinformatics 11, 91-91, (2010).

249 Sigismund, S., Avanzato, D. \& Lanzetti, L. Emerging functions of the EGFR in cancer. Molecular Oncology, n/a-n/a. 
Zhang, W. \& Liu, H. T. MAPK signal pathways in the regulation of cell proliferation in mammalian cells. Cell Research 12, 9, (2002).

Angel, P., Szabowski, A. \& Schorpp-Kistner, M. Function and regulation of AP-1 subunits in skin physiology and pathology. Oncogene 20, 2413, (2001).

Nonami, A. et al. Spred-1 Negatively Regulates Interleukin-3-mediated ERK/Mitogen-activated Protein (MAP) Kinase Activation in Hematopoietic Cells. Journal of Biological Chemistry 279, 52543-52551, (2004).

Felfly, H. \& Klein, 0. D. Sprouty genes regulate proliferation and survival of human embryonic stem cells. 3, 2277, (2013).

Huang, C.-Y. \& Tan, T.-H. DUSPs, to MAP kinases and beyond. Cell \& Bioscience 2, 24-24, (2012).

Sun, L. \& Carpenter, G. Epidermal growth factor activation of NF-kappaB is mediated through IkappaBalpha degradation and intracellular free calcium. Oncogene 16, 2095-2102, (1998).

Liebermann, D. A. \& Hoffman, B. Gadd45 in stress signaling. Journal of Molecular Signaling 3, 15-15, (2008).

Li, T. et al. DDIT3 and KAT2A Proteins Regulate TNFRSF10A and TNFRSF10B Expression in Endoplasmic Reticulum Stress-mediated Apoptosis in Human Lung Cancer Cells. Journal of Biological Chemistry 290, 11108-11118, (2015).

Feng, Z. \& Weinberg, A. Role of bacteria in health and disease of periodontal tissues. Periodontology 2000 40, 50-76, (2006).

Hooper, L. V. Do symbiotic bacteria subvert host immunity? Nat. Rev. Microbiol. 7, 367-374, (2009).

Bolstad, A. I., Jensen, H. B. \& Bakken, V. Taxonomy, biology, and periodontal aspects of Fusobacterium nucleatum. Clinical Microbiology Reviews 9, 55-71, (1996).

Han, Y. W. et al. Interactions between Periodontal Bacteria and Human Oral Epithelial Cells: Fusobacterium nucleatum Adheres to and Invades Epithelial Cells. Infection and Immunity 68, 3140-3146, (2000).

Rubinstein, M. R. et al. Fusobacterium nucleatum promotes colorectal carcinogenesis by modulating E-cadherin/ $\beta$-catenin signaling via its FadA adhesin. Cell host \& microbe 14, 195-206, (2013).

Rawlings, J. S., Rosler, K. M. \& Harrison, D. A. The JAK/STAT signaling pathway. Journal of Cell Science 117, 1281, (2004).

Majérus, M. A., Bibollet-Ruche, F., Telliez, J. B., Wasylyk, B. \& Bailleul, B. Serum, AP-1 and Ets-1 stimulate the human ets-1 promoter. Nucleic Acids Research 20, 2699-2703, (1992).

Dittmer, J. The Biology of the Ets1 Proto-Oncogene. Molecular Cancer 2, 29, (2003).

Li, R., Pei, H., Watson, D. K. \& Papas, T. S. EAP1/Daxx interacts with ETS1 and represses transcriptional activation of ETS1 target genes. Oncogene 19,745-753, (2000).

Francisco-Cruz, A. et al. Granulocyte-macrophage colony-stimulating factor: not just another haematopoietic growth factor. Medical Oncology 31, 774, (2013).

Mann, A., Niekisch, K., Schirmacher, P. \& Blessing, M. Granulocyte-Macrophage Colony-Stimulating Factor Is Essential for Normal Wound Healing. Journal of Investigative Dermatology Symposium Proceedings 11, 87-92, (2013).

Kessler, D. J., Duyao, M. P., Spicer, D. B. \& Sonenshein, G. E. NF-kappa B-like factors mediate interleukin 1 induction of c-myc gene transcription in fibroblasts. The Journal of Experimental Medicine 176, 787, (1992).

Uphoff, C. C. \& Drexler, H. G. Comparative PCR analysis for detection of mycoplasma infections in continuous cell lines. In vitro cellular \& developmental biology. Animal 38, 79-85, (2002).

Drexler, H. G. \& Uphoff, C. C. Mycoplasma contamination of cell cultures: Incidence, sources, effects, detection, elimination, prevention. Cytotechnology 39, 75-90, (2002).

Uphoff, C. C. \& Drexler, H. G. Detection of mycoplasma contaminations. Methods in molecular biology 946, 1-13, (2013).

Sharma, G. D., He, J. \& Bazan, H. E. p38 and ERK1/2 coordinate cellular migration and proliferation in epithelial wound healing: evidence of cross-talk activation between MAP kinase cascades. The Journal of biological chemistry 278, 21989-21997, (2003).

Wang, H. et al. Insulin-like growth factor-1 induces the phosphorylation of PRAS40 via the PI3K/Akt signaling pathway in PC12 cells. Neuroscience letters 516, 105-109, (2012).

Angelo, M. et al. Multiplexed ion beam imaging of human breast tumors. Nature Medicine 20, 436, (2014).

Baharani, A., Trost, B., Kusalik, A. \& Napper, S. Technological advances for interrogating the human kinome. Biochemical Society transactions 45, 65-77, (2017).

McManus, M. T. \& Sharp, P. A. Gene silencing in mammals by small interfering RNAs. Nature Reviews Genetics 3, 737, (2002).

Shalem, 0., Sanjana, N. E. \& Zhang, F. High-throughput functional genomics using CRISPR-Cas9. Nature Reviews Genetics 16, 299, (2015). Jinek, M. et al. A Programmable Dual-RNA-Guided DNA Endonuclease in Adaptive Bacterial Immunity. Science 337, 816, (2012).

Cong, L. et al. Multiplex Genome Engineering Using CRISPR/Cas Systems. Science 339, 819, (2013).

Hayashi, F. et al. The innate immune response to bacterial flagellin is mediated by Toll-like receptor 5. Nature 410, 1099, (2001).

Scholzen, T. \& Gerdes, J. The Ki-67 protein: From the known and the unknown. Journal of Cellular Physiology 182, 311-322, (2000). 
Heyer, W.-D., Ehmsen, K. T. \& Liu, J. Regulation of Homologous Recombination in Eukaryotes. Annual Review of Genetics 44, 113-139, (2010). Chiruvella, K. K., Liang, Z. \& Wilson, T. E. Repair of Double-Strand Breaks by End Joining. Cold Spring Harbor Perspectives in Biology 5, (2013). Maruyama, T. et al. Increasing the efficiency of precise genome editing with CRISPR-Cas9 by inhibition of nonhomologous end joining. Nature Biotechnology 33, 538, (2015).

Chu, V. T. et al. Increasing the efficiency of homology-directed repair for CRISPR-Cas9-induced precise gene editing in mammalian cells. Nature Biotechnology 33, 543, (2015).

Meyle, J., Güttig, K., Rascher, G. \& Wolburg, H. Transepithelial electrical resistance and tight junctions of human gingival keratinocvtes. Journal of Periodontal Research 34, 214-222, (1999).

Kavanagh, E., Tsapara, A., Matter, K. \& Balda, M. S. in Tight Junctions 101-115 (Springer US, 2006).

Anderson, J. M. Molecular Structure of Tight Junctions and Their Role in Epithelial Transport. Physiology 16, 126, (2001).

Fanning, A. S., Ma, T. Y. \& Anderson, J. M. Isolation and functional characterization of the actin-binding region in the tight junction protein Z0-1. The FASEB Journal, (2002).

Groger, S., Michel, J. \& Meyle, J. Establishment and characterization of immortalized human gingival keratinocyte cell lines. Journal of Periodontal Research 43, 604-614, (2008).

Furuse, M. et al. Occludin: a novel integral membrane protein localizing at tight junctions. The Journal of Cell Biology 123, 1777, (1993).

Furuse, M. et al. Direct association of occludin with Z0-1 and its possible involvement in the localization of occludin at tight junctions. The Journal of Cell Biology 127, 1617, (1994).

4 Sanchez de Medina, F., Romero-Calvo, I., Mascaraque, C. \& Martinez-Augustin, 0. Intestinal inflammation and mucosal barrier function. Inflammatory bowel diseases 20, 2394-2404, (2014).

Clevers, H. Modeling Development and Disease with Organoids. Cell 165, 1586-1597, (2016).

Hisha, H. et al. Establishment of a Novel Lingual Organoid Culture System: Generation of Organoids Having Mature Keratinized Epithelium from Adult Epithelial Stem Cells. Scientific Reports 3, 3224, (2013).

Prodan, A. et al. Interindividual variation, correlations, and sex-related differences in the salivary biochemistry of young healthy adults. Eur $J$ Oral Sci 123, 149-157, (2015).

Artis, D. Epithelial-cell recognition of commensal bacteria and maintenance of immune homeostasis in the gut. Nature Reviews Immunology 8 , 411, (2008).

Kostic, A. D. et al. Fusobacterium nucleatum potentiates intestinal tumorigenesis and modulates the tumor-immune microenvironment. Cell host \& microbe 14, 207-215, (2013).

Rubinstein, M. R. et al. Fusobacterium nucleatum promotes colorectal carcinogenesis by modulating E-cadherin/beta-catenin signaling via its FadA adhesin. Cell host \& microbe 14, 195-206, (2013).

Han, Y. W. et al. Identification and characterization of a novel adhesin unique to oral fusobacteria. Journal of bacteriology 187, 5330-5340, (2005).

Fardini, Y. et al. Fusobacterium nucleatum adhesin FadA binds vascular endothelial cadherin and alters endothelial integrity. Molecular microbiology 82, 1468-1480, (2011).

Mishra, A. et al. The Actinomyces oris type 2 fimbrial shaft FimA mediates co-aggregation with oral streptococci, adherence to red blood cells and biofilm development. Molecular microbiology 77, 841-854, (2010).

Fontaine, L. et al. A Novel Pheromone Quorum-Sensing System Controls the Development of Natural Competence in Streptococcus thermophilus and Streptococcus salivarius. Journal of bacteriology 192, 1444-1454, (2010).

Siegl, T. \& Luzhetskyy, A. Actinomycetes genome engineering approaches. Antonie van Leeuwenhoek 102, 503-516, (2012).

Janus, M. M. et al. A novel compound to maintain a healthy oral plaque ecology in vitro. Journal of oral microbiology 8, 32513, (2016).

Janus, M. M. et al. In vitro phenotypic differentiation towards commensal and pathogenic oral biofilms. Biofouling 31, 503-510, (2015).

Prodan, A. et al. A Study of the Variation in the Salivary Peptide Profiles of Young Healthy Adults Acquired Using MALDI-TOF MS. PLoS ONE 11, e0156707, (2016).

van Nes, E. H. et al. What Do You Mean, 'Tipping Point'? Trends in Ecology \& Evolution 31, 902-904, (2016).

Scheffer, M. et al. Early-warning signals for critical transitions. Nature 461, 53, (2009).

Scheffer, M. et al. Anticipating Critical Transitions. Science 338, 344, (2012).

Lahti, L., Salojärvi, J., Salonen, A., Scheffer, M. \& de Vos, W. M. Tipping elements in the human intestinal ecosystem. Nature Communications 5 , 4344, (2014).

Kolenbrander, P. E., Palmer Jr, R. J., Periasamy, S. \& Jakubovics, N. S. Oral multispecies biofilm development and the key role of cell-cell distance. Nature Reviews Microbiology 8, 471, (2010). 
314 Kettle, H., Louis, P., Holtrop, G., Duncan, S. H. \& Flint, H. J. Modelling the emergent dynamics and major metabolites of the human colonic microbiota. Environ Microbiol 17, 1615-1630, (2015).

315 Widder, S. et al. Challenges in microbial ecology: building predictive understanding of community function and dynamics. Ismej 10, 2557-2568, (2016).

316 Egland, P. G., Palmer, R. J., Jr. \& Kolenbrander, P. E. Interspecies communication in Streptococcus gordonii-Veillonella atypica biofilms: signaling in flow conditions requires juxtaposition. Proceedings of the National Academy of Sciences of the United States of America 101, 16917-16922, (2004).

317 Edlund, A. et al. Meta-omics uncover temporal regulation of pathways across oral microbiome genera during in vitro sugar metabolism. Ismej $\mathbf{9}$, 2605-2619, (2015).

318 Imangaliyev, S. Multi-view Learning and Deep Learning for Heterogeneous Biological Data to Maintain Oral Health Doctor thesis, Faculty of Dentistry of University of Amsterdam and VU University of Amsterdam, (2016).

319 Zhang, C.-Z. et al. Saliva in the diagnosis of diseases. International Journal of Oral Science 8, 133-137, (2016). 



\section{Summary}

Oral health depends on a complex interplay between the mucosal tissues, physicochemical and microbial components present in the oral cavity. Maintenance of a stable ecosystem is an essential determinant of oral health. However, as a result of a major change in the ecosystem, the stability can be disturbed leading to an increased risk for development of diseases. Epithelial integrity and barrier function are key aspects to support oral health. The oral cavity consists of stratified squamous epithelium that provides a physical barrier that protects the underlying tissues from mechanical or chemical damage, loss of fluids, and invasion from pathogenic bacteria. In order to maintain barrier function, the oral mucosa undergoes constant renewal and repair. When the epithelial integrity is compromised, prompt re-epithelialization is required to restore homeostasis by re-establishment of cell-cell contacts. This thesis describes the development of a quantitative high-throughput scratch assay to study oral re-epithelialization in vitro as well as the influence of different components of the oral ecosystem (i.e. oral commensal bacteria and salivary metabolites) on gingival re-epithelialization.

In Chapter 2, we describe the development and optimization of an automated high-throughput scratch assay that uses a mathematical model to extract biologically relevant parameters that describe re-epithelialization kinetics. The assay was used to screen 39 lactic acid bacteria (LAB) for their influence in re-epithelialization and therefore, assess their potential to promote gingival epithelial re-epithelialization. The results revealed that exposure of the cells to different Streptococcus salivarius strains leads to enhanced re-epithelialization kinetics. In particular, we showed that $\mathrm{S}$. salivarius MS-oral-D6 is able to stimulate re-epithelialization through a secreted serine protease. Furthermore, in Chapter 3, we developed and implemented the Kinetic Re-Epithelialization Analysis Pipeline (KREAP) into a toolbox in Galaxy, providing an opensource, web-based platform for reproducible image processing and data analysis of high-throughput scratch assays. In addition, Chapter $\mathbf{4}$ provides a detailed procedure of the assay using cell lines originating from oral mucosa as well as from skin, which expands the application of the assay to other research fields.

In Chapter 5, we explored the role of the salivary metabolome on oral re-epithelialization using our scratch assay and the metabolite profiles of a subset of 63 unstimulated saliva samples collected from a healthy cohort $(n=61)$ during a twoweek experimental gingivitis study. Elastic net regression with stability selection led to the identification of a metabolite signature consisting of 10 metabolites that were related to the re-epithelialization kinetics observed in vitro. Using this signature, we were able to predict the re-epithelialization capacity of the remaining 242 saliva samples collected during the clinical study. Higher concentrations of certain plasmalogens, diacylglycerol, and amino acid derivatives in saliva were associated with enhanced re-epithelialization capacity. Moreover, the predicted re-epithelialization capacity of the saliva samples was positively correlated with the gingival bleeding scores determined for the participants during the experimental gingivitis challenge. These scores are used as a clinical parameter to assess the inflammatory state of the periodontal tissues and thus, we propose that the identified metabolite signature reflects the intensity of the mucosal interactions with the resident microbiota. In addition, we found that individuals who displayed larger variation of the metabolite signature over time were associated with a higher increase in gingival bleeding scores during the experimental gingivitis challenge, implying that unstable host-microbe interactions and higher variability of the associated metabolite signature may be an indicator for stronger responses under a challenge. 
In Chapter 6, we employed elastic net regression with stability selection to identify operational taxonomical units (OTUs) that were positively and negatively associated with gingival bleeding scores in the participants of a cross-sectional study ( $n=268$ ). Representative species of the identified OTUs were screened for their influence in oral re-epithelialization using the high-throughput scratch assay. The results revealed that the group of bacteria that had a negative association with gingival bleeding significantly increased re-epithelialization kinetics in comparison with the positive associated group. Importantly, most of these associations were confirmed in a different cohort that participated in a two-week experimental gingivitis challenge study. Higher relative abundances of Actinomyces oris/viscosus/naeslundii, Rothia dentocariosa and Veillonella dispar were strongly correlated with lower gingival bleeding scores, whereas higher relative abundances of Porphyromonas catoniae, Selenomonas sputigena, Leptrotichia buccalis and Streptococcus anginosus were associated with increased gingival bleeding. Additionally, we showed that microbial signatures in saliva can potentially be used to assess the risk of an individual to develop oral diseases, such as gingivitis. Chapter 7 explored the differential modulatory mechanisms exerted by Actinomyces oris, Actinomyces viscosus, Veillonella parvula and Fusobacterium nucleatum subsp. animalis on gingival re-epithelialization kinetics. Cell-based assays and transcriptomic analysis of scratched gingival cells revealed that treatment with A. oris, A. viscosus or V. parvula enhanced re-epithelialization kinetics by increasing cell survival through downregulation of the p53 signalling pathway. In contrast, the increased re-epithelialization kinetics observed with F. nucleatum subsp. animalis was characterized by a stronger induction of regulatory genes involved in cell proliferation, apoptosis and innate immunity responses. Lastly, we showed that the stimulatory effects observed on cell migration by $A$. viscosus and $F$. nucleatum subsp. animalis depend on direct bacteria-host cell contact, whereas A. oris and V. parvula probably secrete soluble compounds that act as chemoattractants for gingival cells.

Chapter 8, summarizes and discusses the key findings of this thesis and provides new avenues for further research. This thesis provides insight in the complex interplay between the oral mucosa and different components of the oral ecosystem that may lead to the development of innovative strategies that could support oral health through the maintenance of epithelial integrity and barrier function. Such strategies may involve the development of novel diagnostic tools to predict disease risk as well as the generation of next-generation probiotics or bioactive compounds to stimulate reepithelialization in patients with impaired wound healing. 


\section{Acknowledgements}

When I just started my position as a PhD candidate at HMI, I thought that 4 years was a long, long time. However, looking back, I think that the saying "times flies when you are having fun" does apply to this period of my life because I am now suddenly standing at the end of this challenging journey. Certainly, this would not have been possible without the support and guidance from many people who, in one way or another, have contributed to the completion of this thesis. To all these people, I would like to dedicate the following paragraphs to express my gratitude.

Firstly, I would like to thank my promotor, Michiel Kleerebezem, for giving me the opportunity to embark in this project and for supporting me in every step of the way. I admire you not only as a scientist, but also as a mentor. You have a unique way of supervising with a perfect balance between independence, guidance, critical thinking, encouragement, and all of these with the right amount of fun. I hope that we can continue collaborating in the future so that I can keep learning from you and hopefully one day, I can become a "robot" too! I also want to extend my gratitude to Jerry Wells, for giving me the opportunity to join $\mathrm{HMl}$ and for all the fruitful discussions and valuable suggestions to improve my manuscripts. Peter van Baarlen, I am sincerely grateful for your willingness to teach me how to tackle complex datasets (e.g. transcriptome analyses) with efficacy. Moreover, your prompt (and early in the morning) reaction to provide your input was always greatly appreciated. Jerry and Peter, I am looking forward to work next to you during the coming years in a new exciting and challenging project. I consider myself very lucky to continue working at HM!!

Furthermore, I would like to thank all the members of the Oral Health project (Andrei Prodan, Marleen Janus, Sultan Imangaliyev, Enno Veerman, Henk Brand, Wim Crielaard, Bastiaan Krom, Evgeni Levin, Bruno Loos, Bart Keijser, Egija Zaura, Bernd Brandt, Jolanda Kool, Ferry Jagers, Elena Nicu, and Michiel Kleerebezem) for your hard work and dedication to fulfil the objectives of this project. I would like to extend my gratitude to Margreet Heerikhuisen and Rianne Hermus for taking care of all the administrative procedures concerning the project. In particular, I want to thank my fellow PhD colleagues, Andrei, Marleen and Sultan, for all the scientific discussions and collaborations as well as your words of support during the most challenging periods of my PhD. I also enjoyed our "non-scientific" meetings a lot, especially when they took place at the Efteling or the Vlaamse Reus. I hope that we stay in contact in the future now that we are all officially holding our PhD degrees!

Over the years, I was fortunate to meet a number of people who provided me with technical support and advice in diverse fields of expertise and who I would like to thank here: Jacques Vervoort, for teaching me how to prepare my samples for NMR measurements and for sharing your vast knowledge on the subject; Sjef Boeren and Berdien van Olst, for guiding me through the "protein world" and helping me with the interpretation of the LC-MS/MS results; Alexa Laheij, for sharing your experience on performing scratch assays with P. gingivalis; Peter Roosjen, for implementing the model inversions in $\mathrm{R}$ and in general, for showing me how scripting can greatly ease my work. Andrew Stubbs and David Zessen, thanks for making KREAP a reality! I enjoyed our meetings very much, and I hope we can continue collaborating in the future. Sultan Imangaliyev, your machine learning skills were key in the project, thanks for understanding how biologists think and providing us with comprehensible explanations; Andrei Prodan, thanks for collecting and aliquoting hundreds of saliva samples and in general, for all the interesting discussions we had over the years; Bernd Brandt, I appreciate your critical input on the microbiota analysis as well as your support during the last stretch of my PhD. Jos Boekhorst, thanks for teaching me how to use Canoco and interpret RDA plots. You always showed great enthusiasm 
and disposition to collaborate and these are characteristics that I sincerely appreciate from you. I look forward to having another opportunity to work together. Thanks to Mark Boekschoten and Jenny Jansen for your technical support and expertise on microarray scanning as well as Juri Matualatupauw for all the discussions we had on transcriptomics. Your thesis served as inspiration for my last experimental chapter! Finally, I would like to thank our technicians: Anja Taverne, for sharing your expertise on microscopy with me, your experience and helpful tips were always appreciated; Nico Taverne, for your practical remarks and your ability to fix nearly everything (especially the BD Pathway!); and Ellen Kranenbarg-Stolte, for your help performing cytokine measurements with the MAGPIX System, your assistance with the cellZscope, and the many, many purchase orders you made for me throughout the years.

As a PhD candidate, I had the pleasure to supervise a total of seven students: Maarten Agelink, Eveline Ultee, Kasper ter Horst, Bob van den Berg, Kim Duintjer, Lisa Robbers, and Franka Siewers. Your hard work, curiosity, and dedication were crucial for this thesis. Guiding each of you during your BSC / MSc thesis was a rewarding experience and I am thankful for all the time we got to spend together working as a team. I am also pleased to see that some of you were motivated to continue in science and are currently busy with your own PhDs. I wish you all success in your future careers!

I was fortunate to be surrounded by amazing colleagues who were crucial during all these years and contributed to the fact that I was able to fully enjoy the ride. Loes, Nico, Anja, Ellen, Raka, Jori, Bart, Bruno, Agnieszka, Rogier, Trudy, Berdien, Nirupama, Nuning, Soumya, Avis, Jonna, Kim, I-Chiao, Simon, Edoardo, Linda, Jurgen, Juri, Annick, Marjolein, Nadya, Stefan, and Laura, thanks for all the gezellige activities, international dinners, Sinterklaas celebrations, coffee breaks, lab outings, flow-cabinet conversations, and supportive speeches when required. Loes, you should definitively win the title for best secretary as you always had all the solutions at hand and a characteristic positive attitude. Thanks for making our lives easier! Anja and Nico, apart from being great technicians, you are also an exemplary couple and very welcoming people who always have their door open for anything we needed. Thank you for being as special as you are! Ellen, I admire your sportive spirit and appreciate the fact that you always made an effort to help me practice my Dutch (although we still need hours and hours of practice!). Rogier, the practical joker of the lab, I will always remember all your pranks and the laughter they caused. Thank you and Edo for listening to my "daily" complaints, you both knew exactly how to motivate me! Linda, I always enjoyed our conversations and appreciate your openness and support. Apart from being a fun colleague, you are one with great taste in clothes! Nadya, thanks for always being honest, your ambition, and your eagerness to join the activities organized by the university or the E-wing. Simon, thanks for all your philosophical and rather inspirational thoughts, I am sure your PhD will be a success. Bruno, you were definitively an entertaining officemate who made me laugh with your amusing stories, I am glad that we have you back in the group! To my colleagues in the office next door: Soumya, Nuning, Nirupama, Agnieszka, and Berdien, thanks for the laughter that sometimes breached into our room and made us smile. In particular, I would like to express my gratitude to my officemates who were there for me during the last stretch of my PhD. Raka, you are a great listener and an even better advisor, I am glad to count you as a colleague and as friend. Jori, I am happy to see that our friendship evolved from the first time that we met. Thanks for the jokes, laughter, and silliness that accompanied us during all these years. Lastly, I would like to thank Bart, your peculiar sense of humour and your abilities with Illustrator are greatly appreciated in our office. Thanks for making my dream of becoming an astronaut more real!

I want to extend my gratitude to all the members of CBI and EZO for making of the E-wing, the best place to work at the Zodiac. In particular, I would like to thank Joeri, Danilo, Lieke, Christine, Natalie, Carmen, Olaf, Eva, Marloes, Elsa, 
Uros, Marcel, Maurijn, and Mike, for all the fun activities, dinners, and trips organized over the years. For the ones that are still busy finalising their PhD thesis, I wish you the best of luck and success in your future careers!

Since I arrived to Wageningen, I have met many people with whom I have shared many special moments and developed valuable friendships. Jelke, Annelien, Jeroen, Mia, Amaya, Sander, Daniela, Paolo and Sui, I am glad I found such a great group of friends and that after all these years, we are still hanging out in Wageningen. Thanks for the Friday drinks, dinners, celebrations, and trips to different countries. Let's keep this up. Joost, you were my first Dutch friend and I am glad you taught me everything I needed to know about Wageningen during the AIDs (including all the most popular pubs). Koenito, you are a good friend and a great housemate, I am happy that you did not go too far away from Wageningen and that I can still see you from time to time. Ale Lucatti and Hanna, you are wonderful friends and also great parents. Thanks for always receiving me in your home and for letting me spoil baby Fabián as a real "auntie" would do. Scott, thanks for being there to motivate me during the last period of the PhD, I enjoyed your company and our discussions very much. Don't go away to Utrecht! Elsa, Eva, Raka, Sam, and Uros, thanks for your constant support as well as the nice dinners and chats over the past years. Edo, Valentina, Alexia, Costas, Rogier, Danilo, Thibaut, Raffaele, and Bruno, thanks for all the dinners/parties in Bennekom and Wageningen, you are a fun group to hang out with!

Quiero darle un agradecimiento especial a mi familia por elección: Irene, Carla, Carlitos, Xio, Coen, Fran, Marce, Rafa, Sergio, Made, Vicky, Pieter, Nacho, Roberto and Ale Mora, ustedes han sido mi apoyo constante desde el momento en que llegué a Holanda y la razón por la cual siempre me he sentido en "casa". Gracias por todos los paseítos, cenas, celebraciones, risas, consejos y conversaciones acerca de la vida. Ustedes siempre tendrán un lugar especial en mi corazón y sé que a pesar de la distancia física que nos separa a algunos de nosotros, siempre contaré con su amistad. ¡Los quiero mucho! A mis amigos de Costa Rica que siempre me acompañan a la distancia: Dani, Caty, Adri, Nane, Evie, Ale Masís, Nancy, Monse, Ale Castro, Maco y Juan Pablo, jmuchas gracias por su amistad!

Special thanks to my lovely paranymphs, Edoardo and Carla. Edo you have been there for me since the first day I started the PhD and we have had an amazing time together. Thanks for the daily discussions, for helping me out troubleshooting multiple experiments, for your encouragement, and most importantly, for making me smile with all your jokes and crazy stories. You are a great colleague and friend and I am very happy that we can continue collaborating during our Post-Docs. Thanks for everything and please send my regards to your friend Mariana who always gives you wise advices! Carlita, we came to the Netherlands from Costa Rica on the same day to obtain our MSc degrees and almost 8 years later we are still here, but now with a PhD degree in our hands. Muchas gracias amiga por siempre estar ahí cuando te he necesitado, por todos los cafecitos, los sábados de yoga y mis habladas eternas. Sos una amiga muy especial y una excelente científica. Estoy segura de que en el futuro seguirás cosechando muchos éxitos.

Gracias a toda mi familia en Costa Rica y Estados Unidos (abuelita, hermanos, tías, tíos, primos y por supuesto a mis papás) que siempre me acompañan en el corazón y me dan la motivación para seguir adelante. En especial quiero agradecer a mi mamá que siempre ha estado conmigo en cada paso que doy. Sos una gran inspiración en mi vida y sé que hoy soy quién soy gracias a todos tus esfuerzos. Gracias papi por siempre tenerme presente y por hacerme reír con todas tus locuras. Sos un padre especial y estoy muy feliz de que podamos celebrar este logro juntos. ¡Los quiero mucho y los extraño siempre! Gelukkig heb ik ook een leuke schoonfamilie in Wageningen. Mark, Margriet, Marieke, Paul en Lobke, bedankt voor de lekkere bbq's, dinertjes en gezellige feestjes. Ik ben blij dat jullie mij in de familie hebben opgenomen! 


\section{2 | Acknowledgements}

Finalmente, quiero agradecer a Petercito. Peter, muchas gracias por ser un apoyo constante, por tu eterna paciencia y tu comprensión. Durante todo este tiempo, has sido clave para mantener mi cordura y sé que sin vos esta aventura de obtener mi título de PhD hubiera sido mucho más difícil. Gracias por todo lo que sos y espero que sigamos enfrentando juntos muchas nuevas aventuras. We made it! (:)

Marcela Fernández Gutiérrez 


\section{About the Author}

María Marcela Fernández Gutiérrez was born on August, $10^{\text {th }}, 1984$ in San José, Costa Rica. After completing her elementary and high school studies with great success, she decided to enrol in Biotechnology at the Instituto Tecnológico de Costa Rica. She graduated cum laude from her BSc program in 2008 and thanks to her multi-tasking skills, -one well-known legend, chronicles her way of studying math and classical ballet, listening to Pearl Jam while meticulously explaining her way of organizing pencils by colour - she accumulated working experience at different companies in Costa Rica. Marcela has worked

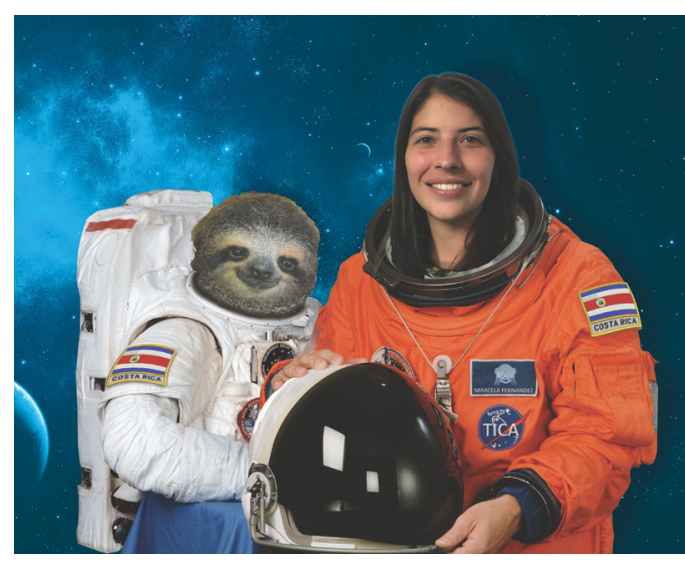
as a genetic laboratory assistant in plant breeding, as a food quality control analyst, and as a sterilization and infection control specialist for the medical devices industry. Furthermore, she also had time to briefly explore her passion for space and extra-terrestrial voyage (see picture just coming back from Slothus Majorus).

However, time was passing by and she had to take the difficult decision to leave her country to go to the beautiful Netherlands to complete her studies. In 2010, she was awarded an NFP fellowship to study the MSc in Biotechnology with the specialization in Medical Biotechnology at Wageningen University. Her thirst for knowledge was insatiable and her love for nature immense. Inspired by her passions, her first thesis was a milestone in the eternal fight between Autographa californica multiple nucleopolyhedrovirus (AcMNPV) and its host. Not yet satisfied, she enlightened her supervisors on the mechanisms of infection used by Infectious Bronchitis Virus (IBV) in primary chicken cells. Marcela's family was immeasurably proud of her for graduating cum laude from her MSc studies (yes, ladies and gentlemen, cum laude again!).

During last five years, Marcela was busy understanding the role of wound healing in oral health and how bacterial species may contribute to accelerate or slow down this process. Thanks to her discoveries in the field of Oral Health, the mouth of millions will be gingivitis-free. As of January 2018, Marcela started working as Postdoc in the Host-Microbe Interactomics group at Wageningen University. Another challenge is in front of her: millions of pigs are in danger, because Streptococcus suis is on the warpath. Marcela is their only hope.

By Edoardo Zaccaria (Paranymph) 


\section{List of Publications}

Kint, J.; Fernandez-Gutierrez, M.; Maier, H.J.; Britton, P.; Langereis, M.A.; Koumans, J.; Wiegertjes, G.F.; Forlenza, M. Activation of the chicken type I interferon response by infectious bronchitis coronavirus. J Virol 89:1156-1167, (2015).

Tarazanova, M.; Beerthuyzen, M.; Siezen, R.; Fernandez-Gutierrez, M.M.; de Jong, A.; van der Meulen, S.; Kok, J.; Bachmann, H. Plasmid Complement of Lactococcus lactis NCD0712 Reveals a Novel Pilus Gene Cluster. PLOS ONE 11(12): e0167970, (2016).

Zaura, E.; Brandt, B.W.; Prodan, A.; Teixeira de Mattos, M.J.; Imangaliyev, S.; Kool, J.; Buijs, M.J.; Jagers, F.L.P.W.; HennequinHoenderdos, N.L.; Slot, D.E.; Nicu, E.A.; Lagerweij, M.D.; Janus, M.M.; Fernandez-Gutierrez, M.M.; Levin, E.; Krom, B.P.; Brand, H.S.; Veerman, E.C.I.; Kleerebezem, M; Loos, B.G.; van der Weijden, G.A.; Crielaard, W.; Keijser, B.J.F. On the ecosystemic network of saliva in healthy young adults. ISME, (2017).

Fernandez-Gutierrez, M.M.; Roosjen, P.P.J.; Ultee, E.; Agelink, M.; Vervoort, J.J.M.; Keijser, B.; Wells, J.M.; Kleerebezem, M. Streptococcus salivarius MS-oral-D6 promotes gingival re-epithelialization in vitro through a secreted protein. Scientific Reports 7(1):11100, (2017).

Zonneveld, M.I.; Fernandez-Gutierrez, M.M.; de Groot, A.M.; van Herwijnen, M.J.C.; Kleinjan, M.; van Capel, T.M.M., Sijts, A.J.A.M.; Taams, L.S.; Garssen, J.; de Jong, E.C.; Kleerebezem, M.; Nolte-'t Hoen, E.N.M.; Redegeld, F.A.; Wauben, M.H.M. Extracellular vesicles in human milk support the epithelial barrier by promoting reepithelialization and modulating innate and adaptive immune responses. To be submitted.

Fernandez-Gutierrez, M.M.; van Zessen, D.B.H.; van Baarlen, P.; Kleerebezem, M.; Stubbs, A.P. KREAP: An automated Galaxy Platform to Quantify Re-epithelialization Kinetics. Submitted for publication.

Fernandez-Gutierrez, M.M.; Roosjen, P.P.J.; van Zessen, D.B.H.; Wells, J.M.; van Baarlen, P.; Stubbs, A.P.; Kleerebezem, M. High-Throughput Screening Model to Quantify Re-Epithelialization Kinetics. To be submitted.

Fernandez-Gutierrez, M.M.; Imangaliyev, S.; Prodan, A.; Loos, B.G.; Keijser, B.; Kleerebezem, M. Salivary metabolite signature predicts in vitro re-epithelialization kinetics and is associated with gingival bleeding in human volunteers. To be submitted.

Fernandez-Gutierrez, M.M., Imangaliyev, S.; Brandt, B.W.; Keijser, B; Loos, B.G.; Kleerebezem, M. Identification of Oral Commensal Bacteria Related to Gingival Bleeding and Re-Epithelialization with Disease-Risk Signature Potential. Manuscript in preparation.

Fernandez-Gutierrez, M.M.; van Baarlen, P.; Robbers, L.; Siewers, F.; Kleerebezem, M. Differential Effects of Oral Bacteria on Gingival Cells Revealed by Cell-Based Assays and Transcriptome Analysis. To be submitted. 


\section{Overview of Completed Training Activities}

\section{Discipline specific activities}

High-Throughput RNAi and Data Analysis, EMBO Heildelberg, Germany 2013

Genetics and physiology of food-associated microorganisms, VLAG, Wageningen, The Netherlands 2013

11th European Oral Microbiology Workshop, Aarhus University, Aarhus, Denmark (Poster presentation)

ALW Platform Molecular Genetics, Lunteren, The Netherlands 2013

Infection meets Immunity (Summer School), UMC Utrecht, Utrecht, The Netherlands 2014

Exploring Human Host-Microbiome Interactions in Health and Disease, Wellcome Trust, Cambridge, United Kingdom (Research Prize Winner) 2015

Statistical Programming in R (Summer School), UMC Utrecht, Utrecht, The Netherlands 2015

Human Immunity and the Microbiome in Health and Disease, Cell Symposia, Montréal, Canada

2015

\section{General courses}

VLAG PhD week, VLAG, Baarlo, The Netherlands 2013

Basic Introduction Course on SPSS, Erasmus Rotterdam, The Netherlands 2013

Basic IP for TIFN researchers, TIFN, Wageningen, The Netherlands 2013

Phylosophy and Ethics of Food Science and Technology, VLAG, Wageningen, The Netherlands 2013

Reviewing a Scientific Paper, Wageningen University, Wageningen, The Netherlands 2013

Information Literacy including EndNote Introduction, Wageningen University, Wageningen, The Netherlands 2014

The Essentials of Scientific Writing and Presenting, Wageningen, The Netherlands 2014

TIFN PhD day on writing and presenting, TIFN, Wageningen, The Netherlands 2015

Project and Time Management, Wageningen University, Wageningen, The Netherlands 2015

Adobe InDesign Essential Training, Wageningen University, Wageningen, The Netherlands 2016

Adobe Illustrator, Wageningen University, Wageningen, The Netherlands 2016

\section{Optional}

Preparation of PhD proposal, Wageningen University, Wageningen, The Netherlands 2012

Kick-off meeting, TIFN, Wageningen, The Netherlands 2012

WE-days, TIFN, Wageningen, The Netherlands $\quad$ 2013-2014

$\begin{array}{lr}\text { Expert meetings, TIFN, Wageningen, The Netherlands } & \text { 2012-2016 }\end{array}$

Work discussion meetings, Wageningen University, The Netherlands $\quad$ 2012-2017 
The study presented in this thesis was performed within the framework of Top Institute Food and Nutrition (TIFN). The work was carried out in the Laboratory of Host-Microbe Interactomics, Wageningen, The Netherlands.

Financial support from Wageningen University for printing this thesis is gratefully acknowledged.

Cover and layout design: Iliana Boshoven-Gkini| AgileColor.com

Printed by: Ridderprint, Ridderkerk, The Netherlands || www.ridderprint.nl 


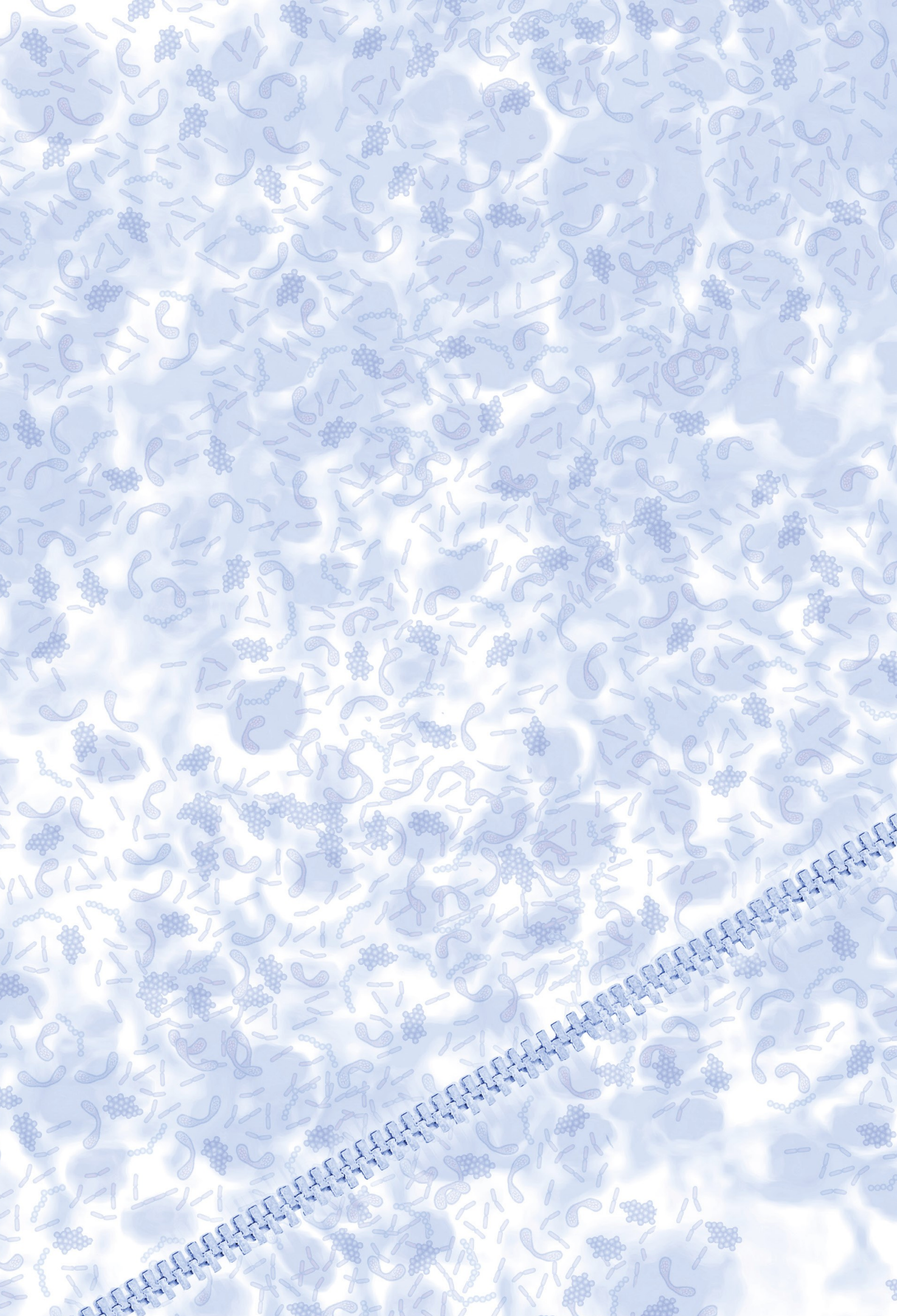

